\title{
Quantum Optics with Diamond Color Centers Coupled to Nanophotonic Devices
}

\section{Permanent link}

http://nrs.harvard.edu/urn-3:HUL.InstRepos:40046485

\section{Terms of Use}

This article was downloaded from Harvard University's DASH repository, and is made available under the terms and conditions applicable to Other Posted Material, as set forth at http:// nrs.harvard.edu/urn-3:HUL.InstRepos:dash.current.terms-of-use\#LAA

\section{Share Your Story}

The Harvard community has made this article openly available.

Please share how this access benefits you. Submit a story.

Accessibility 


\section{HARVARD UNIVERSITY \\ Graduate School of Arts and Sciences}

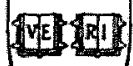

4fs

DISSERTATION ACCEPTANCE CERTIFICATE

The undersigned, appointed by the

Department of Physics

have examined a dissertation entitled

Quantum Optics with Diamond Color Centers Coupled to Nanophotonic Devices

presented by Alp Sipahigil

candidate for the degree of Doctor of Philosophy and hereby certify that it is worthy of acceptance.

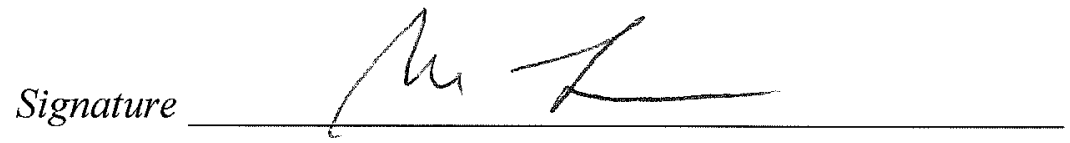

Typed name: Professor Mikhail Lukin, Chair

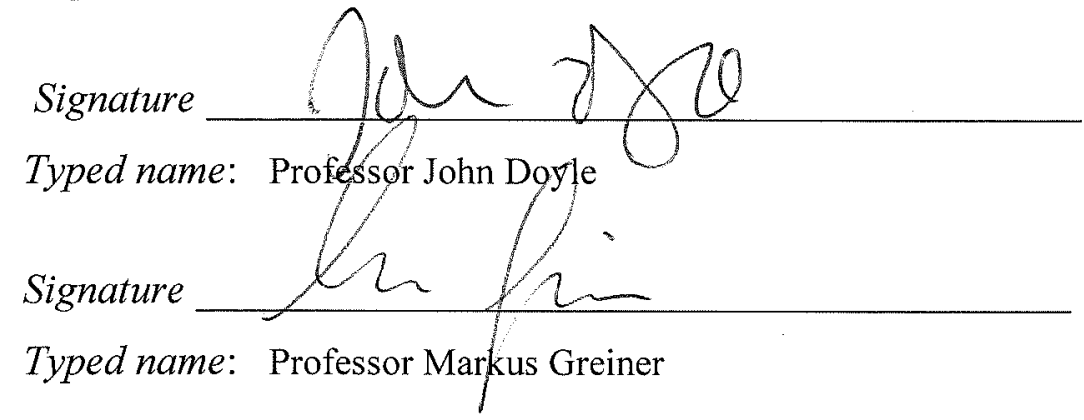

Date: April 10, 2017 



\title{
Quantum Optics with Diamond Color Centers Coupled to Nanophotonic Devices
}

\author{
A dissertation presented
}

by

Alp Sipahigil

to

The Department of Physics

in partial fulfillment of the requirements

for the degree of

Doctor of Philosophy

in the subject of

Physics

Harvard University

Cambridge, Massachusetts

April 2017 
(C)2017 - Alp Sipahigil

All rights reserved. 


\title{
Quantum Optics with Diamond Color Centers Coupled to Nanophotonic Devices
}

\begin{abstract}
Solid-state quantum systems based on Josephson junctions, electronic spins and nuclear spins are among the leading candidates for realizing quantum bits (qubits) in a scalable manner. Despite recent demonstrations of few-qubit quantum registers with these systems, efficient photon-mediated entanglement generation between remote quantum registers remains an outstanding challenge. In this thesis, we develop a platform based on diamond color centers coupled to nanophotonic devices to address this challenge. To realize coherent atom-photon interactions, we first develop an improved understanding of the impact of the solid-state environment on the optical transitions of nitrogen-vacancy (NV) and silicon-vacancy (SiV) color centers in diamond. By investigating the interaction of defect orbitals with phonons and electric fields, we identify the necessary symmetry and temperature conditions at which atomlike, transform-limited optical transitions can be achieved. We show that the inversion symmetry of the $\mathrm{SiV}$ center results in a vanishing static electric dipole moment and protects optical transitions from electric field fluctuations arising from the solid-state environment. The reduced sensitivity to electric field noise results in spectrally-stable optical transitions for $\mathrm{SiV}$ centers, even in nanostructures. We use these properties and bright zero-phonon line emission to efficiently generate indistinguishable photons
\end{abstract}


from separate $\mathrm{SiV}$ centers. These results establish $\mathrm{SiV}$ centers as superior optical emitters compared with NV centers both in terms of brightness and spectral stability. We next integrate $\mathrm{SiV}$ centers into diamond nanophotonic structures and demonstrate strong atom-photon interactions in the high-cooperativity regime of cavity quantum electrodynamics. We use ion implantation to create $\mathrm{SiV}$ centers with coherent optical transitions in diamond photonic crystal cavities. The strongly coupled SiV-cavity system is used to realize a quantum-optical switch that is nonlinear at the single-photon level. Finally, we demonstrate entanglement generation between two SiV centers in a single diamond waveguide based on detection of indistinguishable photons. 


\section{Contents}

Title Page ......................... . .

Abstract . . . . . . . . . . . . . . . . ii

Table of Contents . . . . . . . . . . . . . . . . . $\mathrm{v}$

Citations to Previously Published Work ............. . ix

Acknowledgments . . . . . . . . . . . . . . . . . $\mathrm{x}$

Dedication . . . . . . . . . . . . . . . . . xiii

1 Introduction $\quad 1$

1.1 Background ............................ 1

1.2 Overview of thesis . . . . . . . . . . . . . . . 6

2 Quantum interference of single photons from remote nitrogen-vacancy $\begin{array}{ll}\text { centers in diamond } & 9\end{array}$

2.1 Introduction . . . . . . . . . . . . . . . . . . . . . . . . 9

2.2 Identical photons from two NV centers . . . . . . . . . . . . . . . 12

2.3 Demonstration of HOM interference from two NV centers . . . . . . . 15

2.4 Conclusions and outlook . . . . . . . . . . . . . . . 19

3 Phonon-induced population dynamics and intersystem crossing $\begin{array}{ll}\text { in nitrogen-vacancy centers } & 21\end{array}$

3.1 Introduction . . . . . . . . . . . . . . . . . . . . 21

3.2 Phonon-induced orbital relaxation in NV excited states . . . . . . . . 24

3.3 State-dependent intersystem crossing rates . . . . . . . . . . . . . 27

3.4 Microscopic model of the intersystem crossing process . . . . . . . . . 28

3.5 Conlusions and outlook . . . . . . . . . . . . . . . 33

4 Indistinguishable photons from separated silicon-vacancy centers in $\begin{array}{ll}\text { diamond } & 34\end{array}$

4.1 Introduction . . . . . . . . . . . . . . . . . . . . 34

4.2 Isolating two resonant $\mathrm{SiV}$ centers in a high density ensemble . . . . . 37

4.3 Demonstration of HOM interference from two SiV centers . . . . . . . 39 
4.4 Discussion and outlook . . . . . . . . . . . . . . . . . . . . 42

5 Electron-phonon processes of the silicon-vacancy center in diamond 45

5.1 Introduction . . . . . . . . . . . . . . . . . . . 45

5.2 Experimental results . . . . . . . . . . . . . . . . . . 47

5.2.1 Excited states . . . . . . . . . . . . . . . . . . . . . . . 47

5.2 .2 Ground states . . . . . . . . . . . . . . . . . . 51

5.2 .3 Excited state lifetimes . . . . . . . . . . . . . . . . . . 53

5.2 .4 Optical line positions . . . . . . . . . . . . . 56

5.3 Microscopic model of the electron-phonon processes . . . . . . . . 58

5.3.1 Electron-phonon interaction ............ 59

5.3.2 First-order electron-phonon transitions . . . . . . . . . . . . 60

5.3.3 Second-order electron-phonon transitions . . . . . . . . . . 61

5.3.4 Spin-orbit splitting shifts . . . . . . . . . . . . . 63

5.3.5 Optical line position ................. 64

5.4 Discussion . . . . . . . . . . . . . . . . . . 65

5.4.1 Implications for ground state coherences . . . . . . . . . . 65

5.4.2 Extending ground state coherences . . . . . . . . . . . 67

6 Narrow-linewidth homogeneous optical emitters in diamond nanos$\begin{array}{lr}\text { tructures via silicon ion implantation } & 69\end{array}$

6.1 Introduction . . . . . . . . . . . . . . . . . . 69

6.2 Creating SiV centers with ion implantation . . . . . . . . . . . 74

6.3 Results and discussion . . . . . . . . . . . . . . . . . . . . 75

6.3.1 SiV centers in bulk diamond . . . . . . . . . . . . . . . 75

6.3.2 SiV centers in nanostructures . . . . . . . . . . . . . . 79

7 An integrated diamond nanophotonics platform for quantum optical networks

7.1 Diamond platform for quantum nanophotonics. . . . . . . . . . . . 85

7.2 Quantum-optical switch based on a single SiV center . . . . . . . . . 88

7.3 Tunable single-photon source using Raman transitions. . . . . . . . . 92

7.4 Entanglement of two $\mathrm{SiV}$ centers in a nanophotonic waveguide . . . . 95

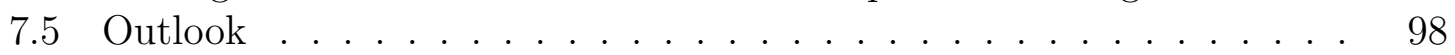

8 Conclusions and outlook $\quad 100$

8.1 Long-lived SiV spin coherence at low temperatures . . . . . . . . . 101

8.2 Photon-mediated two-qubit gates . . . . . . . . . . . . . . 101

8.3 Study of new color centers . . . . . . . . . . . . . . . . . 102 
$\begin{array}{ll}\text { A Supporting material for Chapter 3 } & 105\end{array}$

A.1 Optical Rabi oscillation decoherence measurement . . . . . . . . . . 105

A.2 Rabi decoherence analysis . . . . . . . . . . . . . . . . 108

A.3 Fluorescence depolarization measurement . . . . . . . . . . . . . . . . 109

A.4 Fluorescence depolarization analysis . . . . . . . . . . . . . . 111

A.5 Excited state lifetime measurement . . . . . . . . . . . . . . 113

A.6 Intersystem crossing rate from $\left|\mathrm{E}_{\mathrm{x}}\right\rangle \ldots \ldots \ldots \ldots \ldots \ldots$

A.7 Intersystem crossing rate analysis . . . . . . . . . . . . 117

$\begin{array}{lr}\text { B Supporting material for Chapter 4 } & 119\end{array}$

B.1 Sample information . . . . . . . . . . . . . . . . . . . . . . . . . . . 119

B.2 Inhomogeneous distribution . . . . . . . . . . . . . 120

B.3 Time dynamics of $g^{2}(\tau) \ldots \ldots \ldots \ldots \ldots$

$\begin{array}{lr}\text { C Supporting material for Chapter } 6 & 123\end{array}$

C.1 Experimental setup . . . . . . . . . . . . . . . . . . . . . . . . . . . 123

C.2 Fluorescence autocorrelation measurements . . . . . . . . . . . . 124

$\begin{array}{llr}\text { D } & \text { Supporting material for Chapter } 7 & 126\end{array}$

D.1 Setup description . . . . . . . . . . . . . . . . . . . . 126

D.2 Device design, fabrication and characterization . . . . . . . . . . . . 129

D.2.1 Cavity design . . . . . . . . . . . . . . . . . . . . . . . 129

D.2.2 Cavity fabrication . . . . . . . . . . . . . . . . . . . . 132

D.2.3 Tuning the cavity resonance wavelength _ . . . . . . . . 134

D.2.4 Cavity mode characterization using SiV centers . . . . . . 135

D.2.5 Deterministic SiV positioning using focused Si ion beam implantation . . . . . . . . . . . . . . . 137

D.2.6 Adiabatic fiber-waveguide coupling for high-efficiency photon extraction . . . . . . . . . . . . . . . . . 138

D.3 Identifying single SiV centers inside nanocavities . . . . . . . . . 139

D.4 SiV charge state control: high fidelity initialization and single-shot readout . . . . . . . . . . . . . . . . . . . 141

D.5 Model description for a SiV center inside an optical cavity . . . . . 144

D.5.1 Three level dynamics with dissipation . . . . . . . . . . . 145

D.5.2 System saturation response _. . . . . . . . . . . . 147

D.5.3 Calculation of intensity correlation functions . . . . . . . 148

D.5.4 Extraction of system parameters . . . . . . . . . . . . 151

D.5.5 Measured vs. expected cooperativity . . . . . . . . . . 152

D.6 Subnatural-linewidth Raman single photons . . . . . . . . . . 154

D.7 Experimental procedure for entanglement generation . . . . . . . 157

D.8 Model description for entanglement in a two-SiV system . . . . . . 159

D.8.1 Simple model . . . . . . . . . . . . . . . . . 160 
D.8.2 Detailed model . . . . . . . . . . . . . . . . . . . . 162

D.8.3 Entanglement analysis . . . . . . . . . . . . . . . 164

D.9 Analytical expressions for density matrix elements . . . . . . . . . 171

D.9.1 Steady-state density matrix expressions . . . . . . . . . . 171

D.9.2 Coefficients in the expressions for the time-dependent density matrix elements . . . . . . . . . . . . . . . . . 172

D.9.3 Coefficents and matrix elements for the two-SiV model . . . . 174 


\section{Citations to Previously Published Work}

Chapter 2, in its entirety, has been published as

"Quantum interference of single photons from remote nitrogen-vacancy centers in diamond", A. Sipahigil, M. L. Goldman, E. Togan, Y. Chu, M. Markham, D. J. Twitchen, A. S. Zibrov, A. Kubanek, and M. D. Lukin, Phys. Rev. Lett., 108(14):143601 (2012).

Chapter 3, in its entirety, has been published as

"Phonon-induced population dynamics and intersystem crossing in nitrogenvacancy centers", M. L. Goldman, A. Sipahigil, M. W. Doherty, N. Y. Yao, S. D. Bennett, M. Markham, D. J. Twitchen, N. B. Manson, A. Kubanek, and M. D. Lukin, Phys. Rev. Lett., 114(14):145502, (2015).

Chapter 4 , in its entirety, has been published as

"Indistinguishable photons from separated silicon-vacancy centers in diamond.", A. Sipahigil, K. D. Jahnke, L. J. Rogers, T. Teraji, J. Isoya, A. S. Zibrov, F. Jelezko, and M. D. Lukin, Phys. Rev. Lett., 113(11):113602, (2014).

Chapter 5, in its entirety, has been published as

"Electron-phonon processes of the silicon-vacancy centre in diamond", K. D. Jahnke, A. Sipahigil, J. M. Binder, M. W. Doherty, M. Metsch, L. J. Rogers, N. B. Manson, M. D. Lukin, and F. Jelezko, New J. Phys., 17(4):043011, (2015)

Chapter 6, in its entirety, has been published as

"Narrow-Linewidth Homogeneous Optical Emitters in Diamond Nanostructures via Silicon Ion Implantation", R. E. Evans, A. Sipahigil, D. D. Sukachev, A. S. Zibrov, M. D. Lukin, Phys. Rev. Appl., 5(4):044010 (2016).

Chapter 7 , in its entirety, has been published as

"An integrated diamond nanophotonics platform for quantum-optical networks", A. Sipahigil, R. E. Evans, D. D. Sukachev, M. J. Burek, J. Borregaard, M. K. Bhaskar, C. T. Nguyen, J. L. Pacheco, H. A. Atikian, C. Meuwly, R. M. Camacho, F. Jelezko, E. Bielejec, H. Park, M. Lončar, M. D. Lukin, Science, 354(6314):847-850, (2016). 


\section{Acknowledgments}

First, I thank my advisor Misha Lukin for his continued support and encouragement over the years. His wide knowledge, enthusiasm, intuition and creativity have been a driving force and an inspiration for me. I feel very lucky to have had him as an advisor. Next, I would like to thank John Doyle and Markus Greiner who have been very supportive committee members. I am grateful to John for his help in setting up our dilution fridge experiment and sharing his expertise in low temperature physics. I thank Markus for always being available for consultation and for advice on our experiments.

I was also fortunate to interact with a number of other professors at Harvard and elsewhere. I especially thank Fedor Jelezko who has been instrumental in many of the results presented in this thesis. His expertise, kindness, generosity and openness accelerates progress in our research community and is an inspiration for me. I look forward to continued collaboration with him in the future. The silicon-vacancy (SiV) nanophotonics experiments were built on years of expertise developed in Marko Loncar's and Hongkun Park's groups in diamond nanofabrication. I thank Marko and Hongkun for their support during these experiments. It has been a great experience to work with Marko's team, who can turn physicists' wishlists into devices. I thank Dirk Englund and Mete Atature for many discussions over the years. I am

grateful to Andrei Faraon for hosting me at Caltech for a week of measurements of silicon-vacancy centers in a helium3 cryostat.

One of the greatest things about Misha's group is the number of talented students and postdocs with expertise on a variety of topics. I had the privilege of working with many talented post-docs and students in this stimulating environment. I thank 
Emre Togan for being a great mentor on my first project, steering me in the right direction, teaching me the basics of cryogenic optical spectroscopy and being a great friend. I am grateful to have worked with Mike Goldman and Alex Kubanek on the NV experiments. This was the part of my $\mathrm{PhD}$ where we worked really hard to figure out what is going on in the NV excited states. It was a lot of fun to solve this puzzle together with Mike. These discussions taught me how to understand the microscopics of color centers and were key in our work with silicon-vacancy centers. I thank Yiwen Chu for always being there at times when I most needed support or a simple teabreak. I am grateful to Kay Jahnke who introduced me to the silicon-vacancy center during his visit to Harvard. In the following years, I had the pleasure to work with him and Lachlan Rogers on a number of experiments with this new color center. It has been amazing to be a part of this experience and measure many basic properties for the first time. I thank them for their generosity and being great hosts during my visit to Ulm.

After understanding the basic properties of the $\mathrm{SiV}$, it became clear that we would like to integrate them into nanophotonic devices. Ruffin Evans courageously shared this enthusiasm and provided the device knowledge to start the SiV-nanophotonics effort. Denis Sukachev soon joined the team and was key in setting up the SiVnanophotonics lab. Since then, Denis has continued to amaze me with his wisdom every day. I will not forget the moment when we first observed narrow lines from SiVs in nanostructures. I have been lucky to experience many such moments with Ruffin and Denis who have been great colleagues and friends. I am grateful to Michael Burek for providing us with the state-of-the-art diamond nanophotonic devices, his openness, 
and enthusiasm for pushing the field of diamond quantum nanophotonics forward. In the past two years, I have had the pleasure of working with Christian Nguyen and Mihir Bhaskar. I thank them for their hard work and am looking forward to the many exciting projects they will lead. Over the years, I also had many stimulating discussions and received experimental help from many colleagues: Jeff Thompson, Kristiaan de Greve, Shimon Kolkowitz, Norman Yao, Nathalie de Leon, Sasha Zibrov, Johannes Borregaard, Srujan Meesala, Haig Atikian, Dominik Wild, Marcus Doherty, Peter Maurer, Alexey Akimov, Steve Bennett, Alex High, Quirin Unterreithmeier, Brendan Shields, Alexey Sushkov, Javier Sanchez-Yamagishi, Hannes Bernien, Bo Dwyer, Jan Gieseler, Hannes Pichler, Peter Komar, Michael Gullans, Alexei Bylinskii, Arthur Safira, Liang Jiang, and Jim MacArthur.

This thesis would not be possible without the support of my friends and family over the years. I was blessed to have great friendships that made me feel at home here. I especially feel very lucky to have Olivia Bailey entering my life in this period. Above all, I thank my parents for their continued support and understanding. I dedicate this thesis to them. 
To my parents 


\section{Chapter 1}

\section{Introduction}

\subsection{Background}

The past few decades have witnessed major advances in the isolation and coherent control of individual quantum systems ranging from neutral atoms, ions, and singlephotons to Josephson junction based circuits and electron spins in solids[1, 2]. The ability to control single quantum systems has opened up new possibilities in quantum information processing and simulation $[3,4]$, quantum-enhanced sensing $[5,6]$ and quantum communication $[7,8]$.

Many of these applications require not only the control of isolated quantum bits (qubits), but also controllable interactions between multiple qubits. A variety of techniques have been developed to create such interactions between qubits separated at the sub-millimeter scale. Example approaches include dipole-dipole interactions between neutral atoms $[9,10]$, phonon-mediated interactions in trapped ion crystals $[3$, 11] and photon-mediated interactions in cavity and circuit quantum electrodynamics 
(QED) $[12,13,14]$. The state-of-the-art experiments have used these interactions to entangle up to a few tens of qubits using a bottom up approach[15, 16].

In order to scale these systems up, it will likely be necessary to distribute quantum information between remote nodes that each contain few qubits [8]. This has motivated the development of quantum network nodes where atom-like qubits that can store quantum information are interfaced with optical photons for distributing quantum information[17, 18, 19]. Such nodes also form the basis of quantum repeater architectures for long-distance quantum communication, where atom-like qubits are used as quantum memories for optical photons [20].

Optical interfaces for different qubit architectures are now being actively explored. Microwave-to-optical frequency conversion using mechanical transducers is being investigated to interface microwave qubits based on Josephson junctions with optical photons[19]. For optical emitters such as neutral atoms and trapped ions, cavity QED techniques have been developed to interface hyperfine qubits with optical photons $[7,21]$. In this thesis, we extend these techniques to color centers in diamond and demonstrate key elements required to realize a solid-state quantum network node.

Color centers in diamond are part of a growing list of solid-state optical emitters[22] that include quantum dots[23], rare-earth ions[24], single molecules[25], and point defects in crystals[26]. The recent interest in solid-state emitters for quantum applications builds upon a long history of laser-science, optoelectronics and microscopy research. Since 1960s, transition-metal and rare-earth ions embedded in solids have found wide use as an optical gain medium [27] based on their weakly-allowed optical transitions $[28,29]$. These studies enabled applications ranging from the development 
of optical amplifiers based on erbium-doped silica fibers $\left(\mathrm{Er}^{3+}\right.$ :Silica fiber, gain at $\sim 1550 \mathrm{~nm}$ ) which form the basis of modern telecommunication infrastructure[30] to widely tunable solid-state lasers such as Ti:Sapphire lasers.

These spectroscopic studies of optical emitters in solids were primarily interested in understanding the optical properties and applications of ensembles of dopants and point defects[29, 31]. The desired properties for quantum applications, however, differ strongly from the desired properties of a gain medium. The ability to isolate and control single emitters is critical for realizing a two-level system that can be defined as a qubit. In order to achieve coherent atom-photon interactions based on single solidstate emitters, it is also necessary to identify systems with strong transition dipole moments (to have high radiative decay rates), weak static dipole moments (to minimize optical dephasing due to environmental noise), and weak vibronic coupling (to minimize phonon broadening). In addition, the presence of metastable spin sublevels is necessary to store quantum information.

By early 1990s, advances in optical spectroscopy and microscopy enabled the first major step in this direction, the optical detection of single molecules inside solid-state matrices[32, 25]. In the past two decades, these techniques have been extended to studies of quantum dots and nitrogen-vacancy (NV) color centers in diamond. These systems have shown promising spin and optical properties which made them leading candidates for use as a solid-state quantum network node[33].

Optically active quantum dots are mesoscopic semiconductor structures where electrons and holes are confined to result in a discrete, atom-like optical spectra[23, 34]. Self-assembled InGaAs quantum dots embedded in a GaAs matrix have strong 
optical dipole transitions with excited state lifetimes in the range of $\sim 1 \mathrm{~ns}$. They can be integrated into nanophotonic structures[35] to achieve strong light-matter interactions at very high bandwidths[36, 37]. However, the spatial and spectral positions of these emitters are non-deterministic due to the self-assembly process[35].

Charged quantum dots also have spin degrees of freedom which can be used to store quantum information. The coherence of the optical quantum dot spin is limited by the high density nuclear spin bath in the host crystal which leads to an inhomogeneous spin dephasing timescale of $T_{2}^{*}$ of $\sim 2 \mathrm{~ns}$ and a coherence time $\left(T_{2}\right)$ of 1-3 $\mu$ s using dynamical decoupling sequences[38, 39, 40,41]. The few microsecond long coherence time limits the potential use of InGaAs quantum dots as quantum memories for longdistance quantum communication applications.

The nitrogen-vacancy (NV) color center in diamond consists of a substitutional nitrogen atom with a neighboring vacancy[42, 26]. The NV center has a spin-triplet ground state with a long coherence time of up to $2 \mathrm{~ms}$ at room temperature[43]. Interestingly, the NV spin can be optically polarized and read out at room temperature using off-resonant excitation[44]. The ability to initialize, coherently control and read out the NV spin at room temperature using a simple experimental setup resulted in its wide use in nanoscale sensing applications such as magnetometery and thermometry[45, 46, 47].

At cryogenic temperatures, about $4 \%$ of the NV fluorescence is emitted into a narrowband zero-phonon line (ZPL). For NV centers in bulk diamond, the linewidth of this ZPL transition can be close to the transform-limited linewidth of $13 \mathrm{MHz}$ [48]. For NV centers that are close to surfaces (e.g. in a nanostructure), electric field noise 
due to charge fluctuations in the environment results in spectral diffusion of the optical transitions and non-radiatively broadens the optical transition to few $\mathrm{GHz}[49,50]$. The $4 \%$ emission probability into the ZPL and the factor of $\geq 100$ nonradiative broadening due to spectral diffusion reduce the resonant absorption cross-section of a near-surface NV center by a factor of $\geq 2500$ compared with an ideal two-level optical emitter. This poses a major challenge for realizing a nearly-deterministic spin-photon interface based on an NV center in a microphotonic device. In the past decade, efforts to overcome these challenges focused on integrating NV centers in photonic crystals[51, 49, 52] or fiber-based Fabry-Perot cavities[53, 50] for Purcellenhancing the ZPL emission rate. In addition, methods to minimize the electric field noise originating from surfaces and fabrication-induced damage are being actively explored[54]. In this thesis, we take a different approach and use a different color center, the silicon-vacancy (SiV) center, to address these challenges.

Among the hundreds of color centers in diamond[42], the silicon-vacancy center has received attention from the quantum optics community following the recent demonstration of bright (count rates exceeding 1 million photons per second), narrowband $(\sim 2 \mathrm{~nm})$ single-photon emission at room temperature in Ref.[55]. These properties made the $\mathrm{SiV}$ center the brightest single-photon source in diamond[56]. In Chapters 4,5,6 and 7, we will present a detailed study of the optical coherence properties of the $\mathrm{SiV}$ center at low temperatures and demonstrate coherent atom-photon interactions in the high-cooperativity regime of cavity quantum electrodynamics.

Unlike the NV center whose molecular and electronic structure has been investigated since the 1970s[57], a microscopic model of the SiV center was only very 
recently developed[58,59]. A detailed description of the electronic fine structure of the SiV center can be found in Ref. [60]. Spin properties of the SiV center were recently measured in Refs.[61, 62, 63] which have demonstrated a spin coherence time of about 50-100 ns at $4 \mathrm{~K}$. In Chapter 5, we use cryogenic optical measurements to identify the electron-phonon processes that limit the spin coherence time and develop a microscopic model which predicts that longer spin-coherences can be achieved by operation at lower temperatures.

\subsection{Overview of thesis}

This thesis consists of six experimental projects that are each presented in a separate Chapter. In Chapter 2, we demonstrate that different NV centers can be tuned on resonance to generate indistinguishable photons. Despite the low coherent photon generation rates with NV centers, detection of indistinguishable photons from remote emitters enables distant entanglement generation. Following the publication of the results in this Chapter (see Ref.[64]), this approach was used to demonstrate remote entanglement generation[65] and a loophole-free Bell inequality violation using NV centers[66].

In Chapter 3, we study the interaction of the optical excited states of the NV center with the acoustic phonon bath of diamond. By measuring the phonon-induced relaxation rates between different orbitals, we identify the necessary conditions for high-fidelity indistinguishable photon generation. These studies also provide insights into the microscopic origin of the intersystem-crossing (ISC) which enables optical initialization of the NV spin at room temperature. 
In Chapter 4, we demonstrate that $\mathrm{SiV}$ centers are significantly better compared with NV centers for indistinguishable photon generation. The inversion symmetry of the $\mathrm{SiV}$ center results in a vanishing permanent electric dipole moment for the orbital states and suppresses spectral diffusion. These properties and the bright ZPL transition are used to demonstrate two-photon interference from remote $\mathrm{SiV}$ centers with high coincidence rates.

In Chapter 5, we investigate the phonon-induced electronic dynamics of the $\mathrm{SiV}$ center. We find that the SiV spin strongly interacts with acoustic phonons in diamond and its coherence is limited by electron-phonon interactions at $4 \mathrm{~K}$. We develop a microscopic model of phonon-induced relaxation processes for ground and optically excited states. This model predicts that $\mathrm{SiV}$ spin coherence can be extended by operation at sub-500 $\mathrm{mK}$ temperatures.

In Chapter 6, we show that $\mathrm{SiV}$ centers with high-quality optical properties can be created using $\mathrm{Si}^{+}$ion implantation. We fabricate nanophotonic devices around the implanted $\mathrm{SiV}$ centers and show that the spectral properties are maintained in nanostructures. These observations confirm that $\mathrm{SiV}$ optical transitions are robust against environmental noise as predicted in Chapter 4 .

Chapter 7 describes the main results of this thesis. We demonstrate that a single $\mathrm{SiV}$ center in a diamond photonic crystal cavity can reach the high-cooperativity regime of cavity QED where near-deterministic spin-photon interfaces can be realized. We use this platform to realize a quantum-optical switch controlled by a single SiV center. We control the switch using SiV metastable states and observe optical switching at the single-photon level. Next, we use Raman transitions to realize a single- 
photon source with a tunable frequency and bandwidth in a diamond waveguide. By measuring intensity correlations of indistinguishable Raman photons emitted into a single waveguide, we observe a quantum interference effect resulting from the superradiant emission of two entangled SiV centers. These results are first demonstrations of a high-cooperativity cavity QED experiment with a color center and photon-mediated entanglement generation between two emitters in a single nanophotonic device.

We conclude in Chapter 8 with a discussion of current and future research directions to improve the performance of $\mathrm{SiV}$ centers as well as studies of new color centers. 


\title{
Chapter 2
}

\section{Quantum interference of single}

\author{
photons from remote
}

\section{nitrogen-vacancy centers in}

\section{diamond}

\section{$2.1 \quad$ Introduction}

The interference of two identical photons impinging on a beamsplitter leads to perfect coalescence where both photons leave through the same output port. This fundamental effect, known as Hong-Ou-Mandel (HOM) interference [67], is a consequence of bosonic statistics for indistinguishable particles. HOM interference has been demonstrated using single photon pairs from parametric down-conversion [67] and delayed photons from a single photon source [34, 68, 69]. HOM interference has 
Chapter 2: Quantum interference of single photons from remote nitrogen-vacancy centers in diamond

recently drawn attention as a resource for entanglement generation between distinct single-photon emitters with many potential applications in quantum information science [70]. The effect has been observed for photons emitted by pairs of atoms [71] and trapped ions [72], and has been used for entanglement generation of remote trapped ions [73]. While isolated atoms and ions, which are nominally identical, are a natural source of indistinguishable photons, extending these ideas to condensed matter systems can be challenging since two solid-state emitters are generally distinguishable because of their different local environments. This chapter demonstrates quantum interference of two photons produced by nitrogen-vacancy (NV) impurities in distinct diamond samples separated by two meters. Complementing the recent work involving other solid-state systems $[74,75,76,77]$, the present solid-state realization is particularly significant, since electronic and nuclear spins associated with NV centers can be used as a robust solid-state qubit memory, yielding potential scalable architectures for quantum networks $[78,79]$. Specifically, in combination with a recent demonstration of spin-photon entanglement [80], the present work paves the way for entanglement generation between remote solid-state qubits.

Unlike those associated with atoms in free space, the optical properties of NV centers embedded in a solid state vary substantially from emitter to emitter, especially in distinct samples. This inhomogeneity is due to variation in the local environments of NV centers and, in particular, to variation in the local strain. Furthermore, coincidence experiments are limited by the collection efficiency for light emitted by the NV center. While a wide variety of approaches are currently being explored to enhance the collection efficiency $[81,82,51,83]$, we here utilize solid immersion lenses (SILs) 
Chapter 2: Quantum interference of single photons from remote nitrogen-vacancy centers in diamond

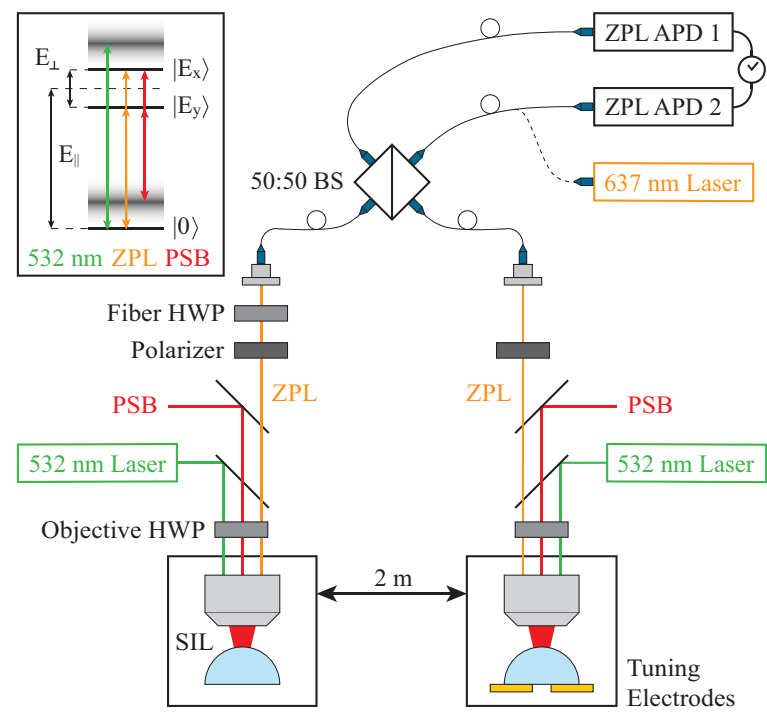

Figure 2.1: Schematic of the apparatus. Two diamond SILs containing multiple NV centers are housed in continuous helium flow cryostats $2 \mathrm{~m}$ apart. Each SIL is addressed by a separate confocal microscopy setup, which includes an arm for excitation at $532 \mathrm{~nm}$, a collection arm for the phonon sideband (PSB), and a collection arm for the zero-phonon line (ZPL). The ZPL collection arm is coupled through a half-wave plate (HWP) into a polarization-maintaining single-mode 50:50 fiber beamsplitter. The two beamsplitter output arms are connected to a pair of avalanche photodiodes (APDs), completing the Hanbury Brown and Twiss detection setup. An excitation laser at $637 \mathrm{~nm}$ is connected in place of ZPL APD 2 to acquire the absorption scan spectra. Electrodes for electric field tuning are installed in one cryostat. The inset shows a simplified level structure, including non-resonant excitation into the excited state PSB at $532 \mathrm{~nm}$, emission into the ground state PSB, and resonant excitation and emission into the ZPL at $637 \mathrm{~nm}$.

fabricated from bulk diamond [84] to facilitate the efficient collection of narrowband photons with identical properties from distant diamond samples. The SILs improve the collection efficiency in the relevant frequency range by an order of magnitude by minimizing total internal reflection at the air-diamond interface, which is significant because of the high refractive index $\left(n_{d}=2.4\right)$ of the diamond host. Very recently, microfabricated SILs have been used to observe HOM interference from two NV centers separated by roughly $20 \mu \mathrm{m}$ on the same diamond chip [74]. 
Chapter 2: Quantum interference of single photons from remote nitrogen-vacancy centers in diamond

In our experiment, we use two 1.0-mm diameter SILs that are fabricated from bulk electronic grade diamond and cut along the (100) crystal plane. The SILs are placed in continuous flow helium cryostats that are separated by two meters, as shown in Fig. 2.1. Resonant excitation with an external-cavity diode laser at $637 \mathrm{~nm}$ and fluorescence detection of PSB emission reveal linewidths in the range of 50-250 $\mathrm{MHz}$ for individual transitions of NV centers in both SILs. These are comparable to narrowest linewidths observed in both synthetic and natural bulk diamond samples $[85,48]$.

\subsection{Identical photons from two NV centers}

To obtain identical photons from two NV centers, the NV centers need to have transitions that are spectrally overlapping, and the emission from these individual transitions for each NV needs to be isolated. By performing simultaneous absorption scans on NV centers in the two SILs with a single laser, we can directly measure the relative detuning of their optical transitions. In our experimental sequence, a $5 \mu \mathrm{s}$ pulse of green light initializes the NV center into the electronic spin sublevel of the triplet ground state with $m_{s}=0(|0\rangle)[86]$. Therefore, we only collect fluorescence from the NV center when the laser is resonant with transitions from the $|0\rangle$ state to the $\left|E_{\mathrm{x}}\right\rangle$ or $\left|E_{\mathrm{y}}\right\rangle$ states, as shown in inset of Fig. 2.1 [87]. We select a pair of NV centers such that one transition in the first NV center is resonant with one transition in the second.

For the HOM measurement, we excite the NV centers with green light and we want to collect ZPL emission only from the selected pair of resonant transitions. The 
Chapter 2: Quantum interference of single photons from remote nitrogen-vacancy centers in diamond

linear and orthogonal polarization selection rules of the $|0\rangle \leftrightarrow\left|E_{x}\right\rangle$ and $|0\rangle \leftrightarrow\left|E_{y}\right\rangle$ transitions allow us to select the emission from one of these transitions by inserting linear polarizers into the ZPL collection arms [85] and setting the Objective HWPs, shown in Fig. 2.1, to the correct angles. Because the ZPL collection used to measure the HOM interference and the resonant excitation used to perform the absorption scans follow the same optical path, we can use the absorption scans to set the correct polarization angle for the ZPL collection. Therefore, we can selectively collect photons emitted from the desired transitions under non-resonant excitation with green light.

We next demonstrate control over the optical properties of NV pairs to compensate for strain-induced spectral inhomogeneities. We make use of the DC Stark effect to actively tune the selected transitions into resonance $[48,88]$. Electric fields perpendicular to the NV axis vary the splitting between $\left|E_{\mathrm{x}}\right\rangle$ and $\left|\mathrm{E}_{\mathrm{y}}\right\rangle$ states and parallel fields shift both transitions together, as shown in the inset of Fig. 2.1. This allows complete control over the optical transition frequencies $[89,88]$. In order to apply the desired electric field, we place one of the SILs on top of a silicon wafer deposited with four electrodes, comprised of $40 \mathrm{~nm} \mathrm{Au}$ on a $\mathrm{Cr}$ adhesion layer. The gate geometry is shown in Fig. 2.2(a). We apply a bias voltage, $V_{\text {app }}$, on one of the electrodes while keeping the other three grounded. In Fig. 2.2(b), the $|0\rangle \leftrightarrow\left|E_{\mathrm{x}}\right\rangle$ transition of NV1 (blue) is tuned across the $|0\rangle \leftrightarrow\left|E_{x}\right\rangle$ transition of NV2 (red) by varying the applied voltage $V_{\text {app }}$ from $-30 \mathrm{~V}$ to $50 \mathrm{~V}$. At $V_{\text {app }}=-2.9 \mathrm{~V}$, shown in Fig. $2.2(\mathrm{c})$, the detuning between the two transitions is reduced to $25 \mathrm{MHz}$ from an initial value of $270 \mathrm{MHz}$. We measured linewidths of $85 \mathrm{MHz}$ for NV1, which was tuned, and $217 \mathrm{MHz}$ for NV2, which was not tuned. Similarly, we did not observe a systematic change of the 
Chapter 2: Quantum interference of single photons from remote nitrogen-vacancy centers in diamond
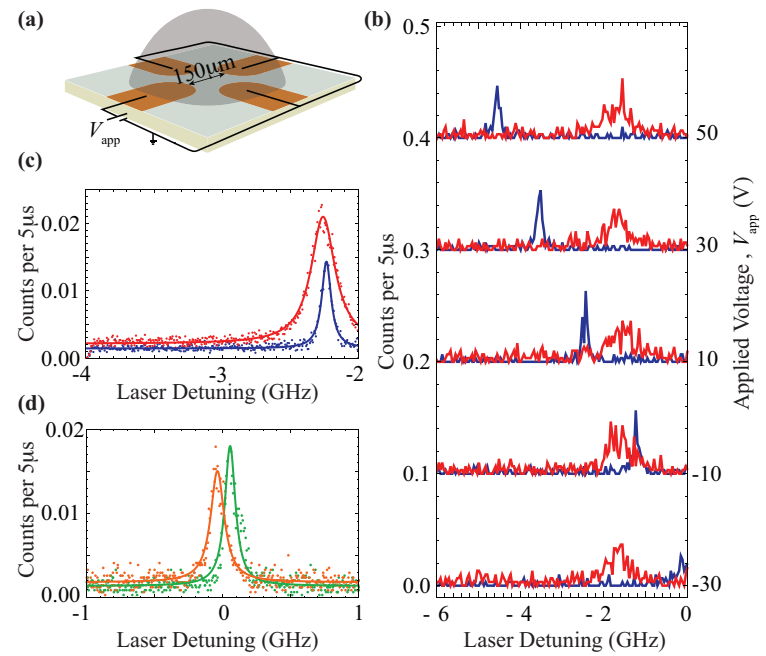

Figure 2.2: Electric field tuning of optical transitions. (a) Four $\mathrm{Cr} / \mathrm{Au}$ gates deposited on silicon. The central gap has a diameter of $150 \mu \mathrm{m}$. In this experiment, only one of the gate voltages was swept while the others were kept grounded. (b) Absorption scans for different applied gate voltages. The gate voltage $V_{\text {app }}$ is varied from -30 to $50 \mathrm{~V}$ for NV1. The $|0\rangle \leftrightarrow\left|\mathrm{E}_{\mathrm{x}}\right\rangle$ transition of NV1 (blue) is tuned across the $|0\rangle \leftrightarrow\left|\mathrm{E}_{\mathrm{x}}\right\rangle$ transition of NV2 (red). For different $V_{\text {app }}$, absorption scan plots are offset by 0.1 for clarity. (c) Linewidth measurement under electric field tuning. On resonance, the measured linewidths are $85 \pm 2 \mathrm{MHz}$ for NV1 (blue) and $217 \pm 4 \mathrm{MHz}$ for NV2 (red). The detuning of the optical transitions in two samples is $25 \pm 2 \mathrm{MHz}$. (d) Linewidth measurement for the NV centers used for the HOM measurement. The measured linewidths are $88 \pm 3 \mathrm{MHz}$ (green) and $106 \pm 4 \mathrm{MHz}$ (orange), and the detuning is $93 \pm 10 \mathrm{MHz}$ without electric field tuning. 
Chapter 2: Quantum interference of single photons from remote nitrogen-vacancy centers in diamond

linewidths with applied external fields in several other NV centers.

Electric field tuning, however, limits the duty cycle when used in combination with green excitation. Here, the green excitation ionizes charge traps in the diamond lattice [88], and these charge dynamics limit the duty cycle during which we can collect fluorescence at the tuned frequency to 50\%. For this reason, for the HOM measurement we selected NV centers whose transitions, shown in Fig. 2.2(d), are inherently detuned by $93 \pm 10 \mathrm{MHz}$ with linewidths of $88 \pm 3 \mathrm{MHz}$ and $106 \pm 4 \mathrm{MHz}$, which eliminates the need for electric field tuning and allows us to run the experiment without reducing the duty cycle.

\subsection{Demonstration of HOM interference from two NV centers}

To demonstrate HOM interference, we apply CW excitation at $532 \mathrm{~nm}$ to the two NV centers whose absorption spectra are shown in Fig. 2.2(d). To confirm that we are addressing one single-photon emitter in each SIL, we infer the normalized, secondorder autocorrelation function $g_{\mathrm{PSB}}^{(2)}(\tau)$ in a standard Hanbury Brown and Twiss setup by splitting the PSB emission in a 50:50 beamsplitter. We expect $g_{\mathrm{PSB}}^{(2)}(0)=0$ for an ideal single-photon source, and the single-photon nature of the emission is confirmed in Figs. 2.3(a,b). We ensure that the ZPL emission from the two NV centers is indistinguishable in frequency through spectral and polarization filtering, as described above. The filtered emission from each NV center is sent to an individual input port of a polarization-maintaining fiber-based beamsplitter. We balance the 

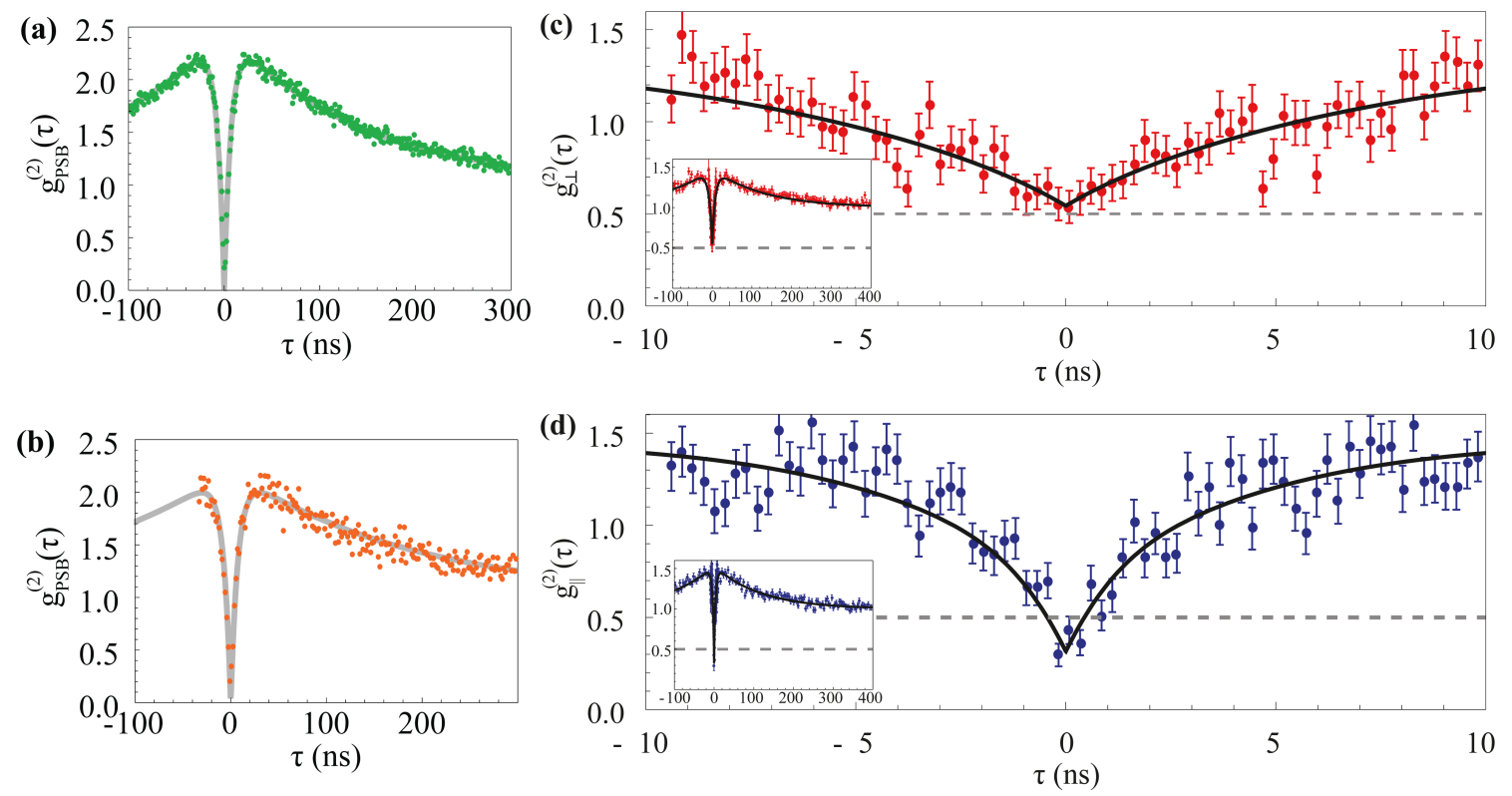

Figure 2.3: (a,b) Single emitter second-order autocorrelation functions, $g_{\mathrm{PSB}}^{(2)}(\tau)$, of the PSB emission, inferred for the two NV centers used for the HOM measurement. (c,d) Demonstration of HOM interference from remote NV centers in the (c) distinguishable case and (d) indistinguishable case. The dashed lines indicate the limit expected from independent distinguishable single photon sources at $\tau=0$. Solid lines are a fit to the data based on the model described in the text. Error bars are estimated based on shot noise. The data is recorded with 64 ps bins, but for presentation has been binned to $192 \mathrm{ps}$ for $|\tau|<10 \mathrm{~ns}$ and $3.84 \mathrm{~ns}$ bins for $|\tau|>10 \mathrm{~ns}$. The data is independently analyzed for both 64 ps bins, and 192 ps bins and the parameter estimates for the fits are in very good agreement. 
Chapter 2: Quantum interference of single photons from remote nitrogen-vacancy centers in diamond

emission intensity by adjusting the green excitation intensity for each NV center independently to obtain 1100 counts per second (cps) per emitter at each output port of the beamsplitter. An additional HWP in one setup is used to adjust the polarization matching of the photons at the beamsplitter. The output ports of the beamsplitter are connected to single photon detectors with timing resolution below 100 ps. The cross-correlation between these detectors is evaluated using a Time-Correlated Single Photon Counting Module with a resolution of 64 ps.

We use the approach described in [75] to analyze the interference data presented in Figs. 2.3(c,d). The expected form of the two-emitter cross-correlation function, $g^{(2)}(\tau)$, is determined by the autocorrelation functions $g_{\mathrm{PSB}}^{(2)}(\tau)$ of the individual emitters, the signal-to-noise ratio, and the degree of distinguishability of photons emitted by the two NV centers. We fix most parameters in our model using independent measurements, as described below, and fit for the visibility of the HOM interference. This visibility, which is extracted from the value of $g^{(2)}(0)$, is a measure of the indistinguishability of the photon pairs. Ideally, $g^{(2)}(0)=0$ for a pair of indistinguishable photons, but the minimal observable $g^{(2)}(0)$ value increases in the presence of experimental noise, as described below. When the photons are distinguishable and the light intensity in both arms is balanced, the correlation measurement will yield $g^{(2)}(0)=0.5[75]$. Measuring the cross-correlation function in the distinguishable case is equivalent to measuring the autocorrelation function of one emitter while the non-interfering emission from the other acts as uncorrelated noise, raising $g^{(2)}(0)$ from 0 to 0.5 . Therefore, a measurement of $g^{(2)}(0)<0.5$ indicates quantum interference between photons emitted by the two single photon sources. 
Chapter 2: Quantum interference of single photons from remote nitrogen-vacancy centers in diamond

Figures 2.3(c,d) show $g^{(2)}(0)$ for two different settings of the HWP angle. In Fig. 2.3(c), the angle is selected such that the emissions from the two NV centers are distinguished by their polarization, yielding $g_{\perp}^{(2)}(0)=0.52 \pm 0.04$. In Fig. 2.3(d), the photons are indistinguishable when their polarizations are parallel, yielding $g_{\|}^{(2)}(0)=$ $0.34 \pm 0.04$. In terms of the visibility of the HOM interference, defined as $\eta=$ $\left[g_{\perp}^{(2)}(0)-g_{\|}^{(2)}(0)\right] / g_{\perp}^{(2)}(0)$, we find $\eta=35 \pm 9 \%$. This $\eta>0$ clearly demonstrates quantum interference between photons emitted by two NV centers separated by $2 \mathrm{~m}$.

We next turn to the detailed discussion of our experimental observations. We first consider the sources of noise that will cause our result to deviate from the ideal case $g_{\|}^{(2)}(0)=0$. The APD dark counts and fluorescence background from our samples will lead to coincidence events, independent of the emission from the NV centers. Background light and the dark counts of our detectors contribute 80 cps out of the total 1100 cps signal, raising $g_{\|}^{(2)}(0)$ to 0.14 . Because the NV center spin is not perfectly polarized under green illumination [86], we expect to collect emission from other transitions (e.g. $\left|A_{2}\right\rangle$ to $\left|m_{s}= \pm 1\right\rangle$ ); since this emission is assumed to be circularly polarized, it is only partially filtered by the polarizer. Emission from other transitions at different frequencies raises the value of $g_{\|}^{(2)}(0)$ by 0.07 . Finally the polarizationmaintaining fiber-based beamsplitters introduce rotations to the polarization of the emission, which increases the distinguishability of the two photons. This contribution raises the $g_{\|}^{(2)}(0)$ value by another 0.07 . Considering these factors, we expect experimental imperfections to raise $g_{\|}^{(2)}(0)$ value to 0.29 , which is in a very good agreement with our experimental observations.

The behavior of the measured $g_{\|}^{(2)}(\tau)$ for $\tau$ longer than $1 / \gamma$, where $\gamma$ is the inverse 
Chapter 2: Quantum interference of single photons from remote nitrogen-vacancy centers in diamond

lifetime of the emitter, is determined solely by the autocorrelation functions for the individual emitters. Using the model described in [90], we extract paremeters from the two-emitter cross-correlation datasets [Figs. 2.3(c,d)] that are in good agreement with those extracted from the single-emitter autocorrelation datasets [Figs. 2.3(a,b)]. The HOM interference only occurs within a window around $\tau=0$ whose width is determined by the bandwidth of the photons emitted from each NV center. In our model, we assume that the emission from the two NV centers is radiatively broadened with bandwidth $\sim \gamma$, and that the center frequencies of the emitted photons are random and different for subsequent emissions. We assume the distribution of the center frequencies is given by the Lorentzian profile that we fit to the absorption spectra shown in Fig. 2.2. Thus the HOM interference has an expected 1/e fullwidth of $2.4 \mathrm{~ns}$. We find that the estimated width agrees with our measured data.

\subsection{Conclusions and outlook}

In summary, we have demonstrated the generation of indistinguishable photons from two spatially separated NV centers. Combined with the recent demonstration of entanglement between the electronic spin of an NV center and the polarization of a photon [80], our work paves the way for optically mediated generation of entanglement between remote solid-state quantum registers. The techniques demonstrated here have yielded improved collection efficiency, control of the NV centers' optical transition frequencies via electric field tuning, and the ability to operate two independent setups simultaneously over three days of continuous data acquisition. The important figure of merit for an entanglement experiment is the time required to 
Chapter 2: Quantum interference of single photons from remote nitrogen-vacancy centers in diamond

generate an entangled pair with fidelity greater than $50 \%$. Considering the resonant excitation scheme used in [80], and noting that it will likely result in stable and narrow optical linewidths [85], we estimate that one entangled spin pair can be created within roughly ten seconds. Improved photon collection techniques that are currently being developed [91] have the potential to increase this generation rate dramatically. Even with the currently estimated rates, though, the exceptionally long nuclear spin memory times of NV centers [92] may allow one to use such systems for the realization of solid-state, multi-node quantum networks. 


\section{Chapter 3}

\section{Phonon-induced population}

\section{dynamics and intersystem crossing}

\section{in nitrogen-vacancy centers}

\subsection{Introduction}

The nitrogen-vacancy (NV) center in diamond has emerged as a versatile atomlike system, finding diverse applications in metrology and quantum information science at both ambient and cryogenic temperatures. At room temperature, the NV center has broad appeal as a sensor- e.g. for nanoscale, biocompatible thermometry [47], magnetometry [93], pressure sensing [94], and electric field sensing [95]. NV centers also have the potential to serve as quantum registers featuring high-fidelity quantum gates [96] and second-long coherence times [92]. All of these applications depend critically on a spin-dependent nonradiative transition into a metastable state, the 

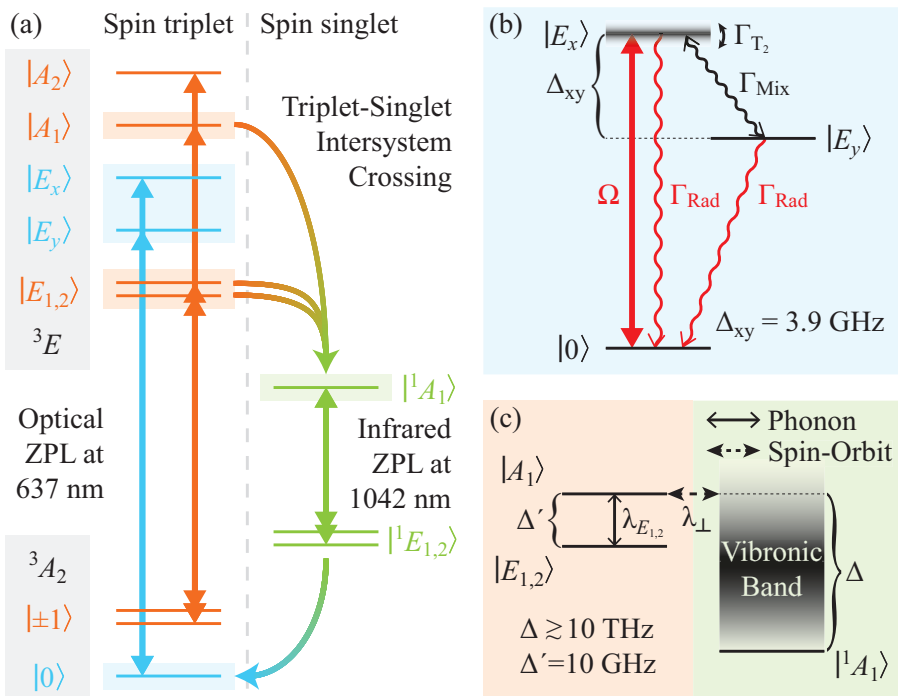

Figure 3.1: The level structure of the NV center. (a) A schematic illustration of the NV center's level structure. The intersystem crossing (ISC) process is responsible for shelving into $\left|{ }^{1} E_{1,2}\right\rangle$ via the short-lived $\left|{ }^{1} A_{1}\right\rangle$ and for pumping into $|0\rangle$. (b) The three-level system of spin-triplet, $m_{s}=0$ states used to model phonon-induced mixing and dephasing within the ${ }^{3} E$ manifold. (c) The states, Hamiltonian matrix elements $\left(\lambda_{E_{1,2}}, \lambda_{\perp}\right)$, and energy scales $\left(\Delta, \Delta^{\prime}\right)$ involved in the triplet-singlet ISC.

so-called intersystem crossing (ISC), which enables nonresonant optical initialization and readout of the electronic spin state. Despite theoretical [97] and experimental $[97,98,99,100]$ efforts, a detailed understanding of the microscopic ISC mechanism has remained an open question. Such an understanding may enable efforts to enhance the NV center's optical initialization and readout fidelities, or to identify or engineer similar mechanisms in other solid-state defects.

In this chapter, we use resonant optical manipulation of an NV center at cryogenic temperatures to probe the NV center's interaction with phonons in the diamond lattice. The NV center has a spin-triplet, orbital-singlet ground state $\left({ }^{3} A_{2}\right)$ that is coupled optically to a spin-triplet, orbital-doublet excited state $\left({ }^{3} E\right)$, as shown in 
Fig. 3.1. We investigate the ISC from the ${ }^{3} E$ manifold to the intermediate spinsinglet states $\left(\left|{ }^{1} A_{1}\right\rangle,\left|{ }^{1} E_{1,2}\right\rangle\right)$ and phonon-mediated population transfer within the ${ }^{3} E$ manifold. We first measure phonon-induced population transfer between $\left|E_{\mathrm{x}}\right\rangle$ and $\left|E_{y}\right\rangle$, using the effectively closed three-level system shown in Fig. 3.1(b), to characterize phononic coupling to the orbital electronic state. We build on previous such measurements $[85,101]$, in which population transfer was not shown to be completely suppressed, by adopting techniques that enable highly coherent excitation of the NV center's optical transitions [102, 65].

We next measure the fluorescence lifetimes of $\left|A_{1}\right\rangle,\left|A_{2}\right\rangle$, and $\left|E_{1,2}\right\rangle$ and find that the ISC rates from these states differ sharply. Based on these experimental observations, we develop a model of the ISC mechanism that combines spin-orbit coupling, phonon-induced electronic state transitions, and phonon-mediated lattice relaxation. Using our measured phonon-induced mixing rate as an input, we find excellent quantitative agreement between our model, our experimental results, and previous observations $[97,98,99,100]$.

In our experiment, we use a $1.0 \mathrm{~mm}$ diameter solid-immersion lens (SIL) that is fabricated from bulk electronic grade CVD diamond and cut along the (100) crystal plane [84]. The SIL is mounted in a continuous flow helium cryostat that allows us to vary the temperature from $4.8 \mathrm{~K}$ to room temperature. We use a laser at $532 \mathrm{~nm}$ for nonresonant initialization of the NV center's charge and spin states, and two tuneable external-cavity diode lasers gated by electro-optical amplitude modulators to apply independent resonant pulses at $637 \mathrm{~nm}$. Using photoluminescence excitation (PLE) spectroscopy, we resolve five of the six dipole-allowed ${ }^{3} A_{2} \rightarrow{ }^{3} E$ transitions; at zero 
Chapter 3: Phonon-induced population dynamics and intersystem crossing in nitrogen-vacancy centers

applied magnetic field and low strain, $\left|E_{1}\right\rangle$ and $\left|E_{2}\right\rangle$ are too close in energy for their transitions from $| \pm 1\rangle$ to be resolved.

\subsection{Phonon-induced orbital relaxation in NV ex- cited states}

To measure the mixing rate between $\left|E_{x}\right\rangle$ and $\left|E_{y}\right\rangle$, we measure both the decay rate out of one of the states and the rate of population transfer between the two states. First, we measure the decay rate out of $\left|\mathrm{E}_{\mathrm{x}}\right\rangle$ by measuring the timescale $\tau_{\text {Rabi }}$ on which optical Rabi oscillations between $|0\rangle$ and $\left|E_{x}\right\rangle$ decohere. We apply resonant $60 \mathrm{~ns}$ pulses and record the arrival times of the resulting phonon sideband (PSB) photons (See Appendix A for details). The resulting optical Rabi oscillations are shown in Fig. 3.2(a).

The optical Rabi oscillation measurement is repeated at different temperatures. For each temperature, we fit $\tau_{\text {Rabi }}$ from the oscillation decay and extract $\Gamma_{\text {Rad }}=1 / \tau_{\text {Rad }}$ from the pulse's falling edge, where $\tau_{\text {Rad }}$ is the radiative lifetime of $\left|E_{x}\right\rangle$. From these two values, we extract

$$
\Gamma_{\text {Add }}=\Gamma_{\text {Mix }}+\Gamma_{T_{2}}=2\left(1 / \tau_{\text {Rabi }}-\frac{3}{4} \Gamma_{\text {Rad }}\right),
$$

the additional decoherence rate of the Rabi oscillations due to processes other than optical decay to $|0\rangle$ [103]. $\Gamma_{\text {Mix }}$ and $\Gamma_{T_{2}}$ are the phonon-induced mixing and dephasing rates, respectively. The observed $T^{5}$ scaling of $\Gamma_{\text {Add }}$ in in Fig. 3.2(a) indicates that the additional Rabi decoherence is due primarily to mixing between $\left|\mathrm{E}_{\mathrm{x}}\right\rangle$ and $\left|\mathrm{E}_{\mathrm{y}}\right\rangle$ mediated by two $E$-symmetric phonons $[104,85]$. We infer $\Gamma_{\text {Add }} / 2 \pi=-0.34 \pm 1.87$ 

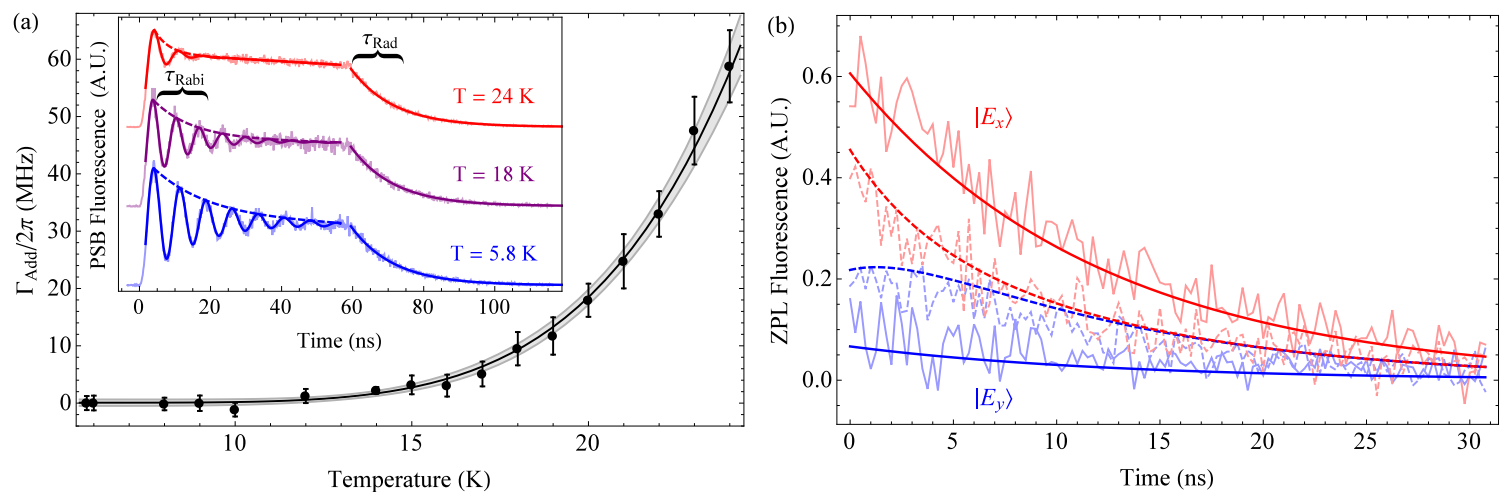

Figure 3.2: Phonon-induced mixing between the $\left|\mathrm{E}_{\mathrm{x}}\right\rangle$ and $\left|\mathrm{E}_{\mathrm{y}}\right\rangle$ electronic orbital states. (a) The measured $\Gamma_{\text {Add }}=\Gamma_{\text {Mix }}+\Gamma_{T_{2}}$ as a function of temperature, with a fit to $\Gamma_{\text {Add }} \propto T^{5}$. The shaded region is the $95 \%$ confidence interval and the inset shows Rabi oscillations on the $|0\rangle \rightarrow\left|\mathrm{E}_{\mathrm{x}}\right\rangle$ transition measured at three temperatures, offset for clarity. (b) Background-subtracted fluorescence of $x$ (red) or $y$ (blue) polarization collected after resonant excitation to $\left|\mathrm{E}_{\mathrm{x}}\right\rangle$. Data were taken at $T=5.0 \mathrm{~K}$ (solid lines) and $T=20 \mathrm{~K}$ (dashed lines). The fits are simulations to the three-level system depicted in Fig. 3.1 and Appendix A.

$\mathrm{MHz}$ at $5.8 \mathrm{~K}$ (95\% confidence interval), indicating that phonon-induced mixing is frozen out at low temperature. There also exists a one-phonon emission process whose contribution to the mixing rate scales as $\Delta_{x y}^{2} T$ [104]. This contribution is negligible in our experiment because of the small density of states for phonons of frequency $\Delta_{x y}=3.9 \mathrm{GHz}^{1}$.

We also measure population transfer between $\left|E_{x}\right\rangle$ and $\left|E_{y}\right\rangle$ directly by measuring the depolarization of the emitted zero-phonon line (ZPL) fluorescence. ZPL photons emitted by decay from the $\left|E_{x}\right\rangle$ and $\left|E_{y}\right\rangle$ states have orthogonal linear polarizations

\footnotetext{
${ }^{1}$ Although the one-phonon process contributes negligibly in this experiment, it may cause significant mixing in NV centers, such as those formed by nitrogen implantation or placed inside nanofabricated structures, where damage to the local crystalline structure may induce a large strain splitting. We note that this linear temperature scaling may be evident in the result of a previous measurement of phonon-induced mixing, which used NV centers with strain splittings of 8 to 81 $\mathrm{GHz}[85]$.
} 


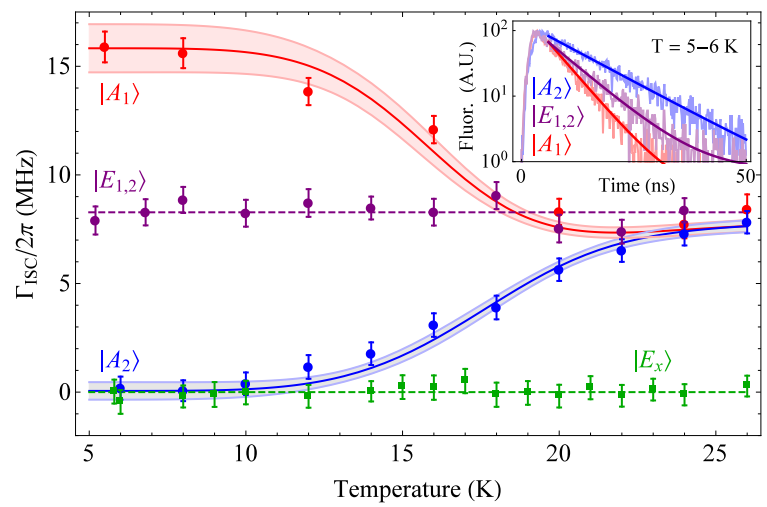

Figure 3.3: ISC rates from the ${ }^{3} E$ triplet excited states. The inset shows the measured PSB fluorescence collected after excitation to $\left|A_{1}\right\rangle,\left|A_{2}\right\rangle$, and $\left|E_{1,2}\right\rangle$ measured at $T \sim 5$ $\mathrm{K}$, normalized to a common peak height and fit to an exponential decay curve. The blue and red bands are fits with $95 \%$ confidence intervals to the phonon-induced mixing model described in the text, and the purple and green lines are placed at the mean values of the corresponding data sets.

(labeled $x$ and $y$ ). At $5 \mathrm{~K}$ and $20 \mathrm{~K}$, we resonantly excite the $\mathrm{NV}$ center to $\left|\mathrm{E}_{\mathrm{x}}\right\rangle$ and collect fluorescence of $x$ and $y$ polarizations, as shown in Fig. 3.2(b). At both temperatures, the emission is $x$-polarized for small delays, indicating initial decay primarily from $\left|\mathrm{E}_{\mathrm{x}}\right\rangle$. The emission remains $x$-polarized at $5 \mathrm{~K}$, whereas we observe that emission becomes depolarized at $20 \mathrm{~K}$. Since emission polarization is directly related to excited state population, this is a direct observation of population transfer between $\left|\mathrm{E}_{\mathrm{x}}\right\rangle$ and $\left|\mathrm{E}_{\mathrm{y}}\right\rangle$. We compare the observed population transfer to simulations of rate equations based on the three-level system depicted in Fig. 3.1(b). Using the values of $\Gamma_{\text {Add }}$ given by the fit in Fig. 3.2(a), and using our polarization selectivity and the starting time of the mixing/radiative decay dynamics relative to the excitation pulse as fit parameters, we find good agreement between the observed and simulated fluorescence depolarization. 


\subsection{State-dependent intersystem crossing rates}

We now turn from the ${ }^{3} E$ states with $m_{s}=0\left(\left|\mathrm{E}_{\mathrm{x}}\right\rangle\right.$ and $\left.\left|\mathrm{E}_{\mathrm{y}}\right\rangle\right)$ to the states with $\left|m_{s}\right|=1\left(\left|A_{1}\right\rangle,\left|A_{2}\right\rangle\right.$, and $\left.\left|E_{1,2}\right\rangle\right)$. Although the radiative decay rate $\Gamma_{\text {Rad }}$ is the same for all ${ }^{3} E \rightarrow{ }^{3} A_{2}$ transitions $[98,99]$, one expects from symmetry arguments that $\left|A_{1}\right\rangle,\left|A_{2}\right\rangle$, and $\left|E_{1,2}\right\rangle$ should exhibit different ISC rates into the spin-singlet states [105]. We can therefore probe population dynamics among these states by exciting the NV center into one state and measuring its fluorescence lifetime as a function of temperature. A representative measurement is shown in the inset to Fig. 3.3. From the fluorescence decay time $\tau_{i}$ measured after excitation into the $i$ th state, we can calculate the associated ISC rate

$$
\Gamma_{i}=1 / \tau_{i}-\Gamma_{\text {Rad }}
$$

Because the ISC rates from $\left|\mathrm{E}_{\mathrm{x}}\right\rangle$ and $\left|\mathrm{E}_{\mathrm{y}}\right\rangle$ are negligible $\left(\Gamma_{E_{x}} / 2 \pi \leq 0.62 \pm 0.21 \mathrm{MHz}\right.$, Appendix A) compared to the ISC rates from $\left|A_{1}\right\rangle,\left|A_{2}\right\rangle$, and $\left|E_{1,2}\right\rangle$, we set $\Gamma_{\text {Rad }} / 2 \pi=$ $1 / 2 \pi \bar{\tau}_{E_{x}}=13.2 \pm 0.5 \mathrm{MHz}$, where $\bar{\tau}_{E_{x}}$ is the average lifetime of $\left|\mathrm{E}_{\mathrm{x}}\right\rangle$ extracted from our Rabi decoherence data. The derived values of $\Gamma_{i}$ are shown in Fig. 3.3.

We observe that the $\left|A_{1}\right\rangle,\left|A_{2}\right\rangle$, and $\left|E_{1,2}\right\rangle$ ISC rates are significantly different at low temperatures, but converge around $T \gtrsim 22 \mathrm{~K}$. The same two-phonon process that redistributes population among $\left|\mathrm{E}_{\mathrm{x}}\right\rangle$ and $\left|\mathrm{E}_{\mathrm{y}}\right\rangle$ also does so among $\left|A_{1}\right\rangle$ and $\left|A_{2}\right\rangle$. As a

result, the observed temperature-dependent ISC rates $\left(\tilde{\Gamma}_{A_{1}}\right.$ and $\left.\tilde{\Gamma}_{A_{2}}\right)$ converge to an average of the two unmixed states' rates $\left(\Gamma_{A_{1}}\right.$ and $\left.\Gamma_{A_{2}}\right)$ as the temperature increases. We fit $\tilde{\Gamma}_{A_{1}}$ and $\tilde{\Gamma}_{A_{2}}$, assuming $\Gamma_{A_{2}}=0$ and a temperature-dependent $\left|A_{1}\right\rangle-\left|A_{2}\right\rangle$ mixing rate equal to the measured $\left|E_{x}\right\rangle-\left|E_{y}\right\rangle$ rate. We find excellent agreement 
Chapter 3: Phonon-induced population dynamics and intersystem crossing in nitrogen-vacancy centers

using only $\Gamma_{A_{1}}$ as a free parameter, confirming that the same phonon-induced mixing process is evident in both Figs. 3.2 and 3.3. The state lifetimes we observe at $T \geq 22$ $\mathrm{K}$ are consistent with those of the $m_{s}=0$ and $\left|m_{s}\right|=1$ states at room temperature [see Fig. 3.4(c)], indicating that we have measured the onset of the orbital averaging mechanism that enables the ${ }^{3} E$ manifold to be treated as an effective spin-triplet, orbital-singlet system at room temperature $[106,107]$.

\subsection{Microscopic model of the intersystem crossing process}

We now present a theoretical analysis of the ISC mechanism, which is treated in greater detail in Ref. [104]. In the NV center, an axial spin-orbit (SO) interaction $\left(\propto \lambda_{\|} l_{z} s_{z}\right)$ is primarily responsible for the fine structure of the ${ }^{3} E$ manifold while a transverse SO interaction $\left[\propto \lambda_{\perp}\left(l_{x} s_{x}+l_{y} s_{y}\right)\right]$ couples states of different spin multiplicities $[108,89]$. The ISC occurs in two steps [see Fig. 3.1(c)]: (1) a SO-mediated transition from a state in the ${ }^{3} E$ manifold to a resonant excited vibrational level of $\left|{ }^{1} A_{1}\right\rangle$, and (2) relaxation of the excited vibrational level to the ground (or thermally occupied) vibrational level of $\left|{ }^{1} A_{1}\right\rangle$. Because the latter occurs on the picosecond timescale [109], the overall ISC rate is defined by the initial SO-mediated transition.

According to Fermi's golden rule, the SO-mediated transition requires both $\mathrm{SO}$ coupling and overlap between the initial vibrational level of ${ }^{3} E$ and the excited vibrational level of $\left|{ }^{1} A_{1}\right\rangle$. Because $\left|{ }^{1} A_{1}\right\rangle$ and the ${ }^{3} A_{2}$ states consist of the same singleparticle electronic orbitals, the two states exhibit similar charge density distributions 
Chapter 3: Phonon-induced population dynamics and intersystem crossing in nitrogen-vacancy centers

and therefore similar vibrational potentials [110]. The vibrational overlap between the ${ }^{3} E$ states and $\left|{ }^{1} A_{1}\right\rangle$ is then well approximated by that observed in the PSB of the ${ }^{3} E \rightarrow{ }^{3} A_{2}$ optical emission spectrum. Selection rules imply that only $\left|A_{1}\right\rangle$ is SO-coupled with $\left|{ }^{1} A_{1}\right\rangle$. Thus, SO coupling can mediate a first-order transition from $\left|A_{1}\right\rangle$ to a resonant excited vibrational level of $\left|{ }^{1} A_{1}\right\rangle$, whereas $\left|E_{1,2}\right\rangle$ must undergo a second-order transition involving electron-phonon coupling with $\left|A_{1}\right\rangle$ [see Fig. 3.1(c)]. In principle, $\left|E_{1,2}\right\rangle$ may undergo a first-order transition to a highly excited vibrational level of $\left|{ }^{1} E_{1,2}\right\rangle$, but we expect the rate of this transition to be negligible because the $\left|{ }^{1} A_{1}\right\rangle-\left|{ }^{1} E_{1,2}\right\rangle$ energy spacing (1190 meV [111]) is large compared to the extent of the PSB $(\sim 500 \mathrm{meV}[57,110])$.

The ISC rate from $\left|A_{1}\right\rangle$ is

$$
\begin{aligned}
\Gamma_{A_{1}} & =4 \pi \hbar \lambda_{\perp}^{2} \sum_{n}\left|\left\langle\chi_{0} \mid \chi_{\nu_{n}}^{\prime}\right\rangle\right|^{2} \delta\left(\nu_{n}-\Delta\right) \\
& =4 \pi \hbar \lambda_{\perp}^{2} F(\Delta)
\end{aligned}
$$

where $\lambda_{\perp}$ is the transverse spin-orbit coupling rate, $\Delta$ is the energy spacing between $\left|A_{1}\right\rangle$ and $\left|{ }^{1} A_{1}\right\rangle$, and $\delta$ is the Dirac delta function. $\left|\chi_{0}\right\rangle$ is the ground vibrational level of $\left|A_{1}\right\rangle$, and $\left|\chi_{\nu_{n}}^{\prime}\right\rangle$ are the vibrational levels of $\left|{ }^{1} A_{1}\right\rangle$ with energies $\nu_{n}$ above that of $\left|{ }^{1} A_{1}\right\rangle$. We define the vibrational overlap function $F(\Delta)=\overline{\left|\left\langle\chi_{0} \mid \chi_{\Delta}^{\prime}\right\rangle\right|^{2}} \rho(\Delta)$, where the average is over all vibrational levels with energy $\Delta$ and $\rho(\Delta)$ is the associated density of states. 
At low temperature, the ISC rate from $\left|E_{1,2}\right\rangle$ is

$$
\begin{aligned}
\Gamma_{E_{1,2}} & =4 \pi \hbar^{3} \lambda_{\perp}^{2} \sum_{n, p, k} \frac{\lambda_{p, k}^{2}}{\omega_{k}^{2}}\left|\left\langle\chi_{0} \mid \chi_{\nu_{n}}^{\prime}\right\rangle\right|^{2} \delta\left(\nu_{n}+\omega_{k}-\Delta\right) \\
& =\frac{2}{\pi} \hbar \eta \Gamma_{A_{1}} \int_{0}^{\min (\Delta, \Omega)} \omega \frac{F(\Delta-\omega)}{F(\Delta)} \mathrm{d} \omega,
\end{aligned}
$$

where $\lambda_{p, k}$ is the phononic coupling rate for a phonon of polarization $p$ and wavevector $k$, and

$$
J(\omega)=\frac{\pi \hbar}{2} \sum_{k} \lambda_{E_{1,2}, k}^{2} \delta\left(\omega-\omega_{k}\right)=\eta \omega^{3},
$$

is the phonon spectral density in the acoustic limit [85] for the polarization that couples $\left|E_{1,2}\right\rangle$ with $\left|A_{1}\right\rangle$. We assume a cutoff energy $\Omega$ for acoustic phonons.

Because the SO interaction is $A_{1}$-symmetric [112], and can therefore only couple states of like symmetry, $\left|A_{2}\right\rangle$ is not SO-coupled to either $\left|{ }^{1} A_{1}\right\rangle$ or $\left|{ }^{1} E_{1,2}\right\rangle[108,89]$. Similar symmetry considerations forbid single-phonon coupling between $\left|A_{2}\right\rangle$ and $\left|A_{1}\right\rangle$, so neither the first- nor second-order processes described above can induce ISC decay from $\left|A_{2}\right\rangle$. The lowest-order allowed mechanism would be a third-order process involving two phonons and one SO interaction, but we neither expect nor observe an appreciable ISC transition rate due to such a high-order process.

In Fig. 3.4(a), we plot the prediction of Eq. 3.3 as a function of $\Delta$ and the value of $\Gamma_{A_{1}} / 2 \pi=16.0 \pm 0.6 \mathrm{MHz}$ extracted from the fits shown in Fig. 3.3. The broad range of predicted values of $\Gamma_{A_{1}}$ arises from the currently imprecise knowledge of the transverse SO coupling rate $\lambda_{\perp}{ }^{2}$. The vibrational overlap function is extracted from a previous measurement of the ${ }^{3} E \rightarrow{ }^{3} A_{2}$ emission PSB [110]. The intersection of

\footnotetext{
${ }^{2}$ The axial SO coupling rate $\lambda_{\|}=5.33 \pm 0.03 \mathrm{GHz}$ is known [113], but the precise value of $\lambda_{\perp} / \lambda_{\|} \approx 1$ remains an open question. As explained in Ref. [104], we have selected $\lambda_{\perp} / \lambda_{\|}=1.2 \pm 0.2$ as a reasonable confidence interval.
} 
(a)

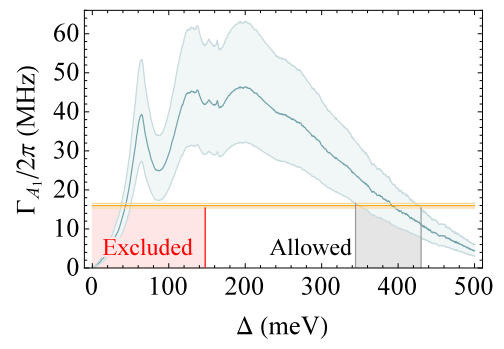

(b)

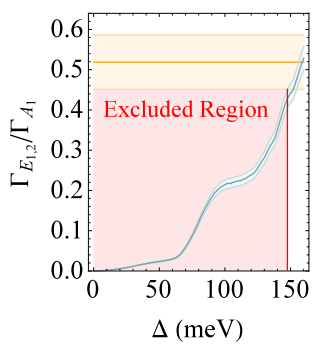

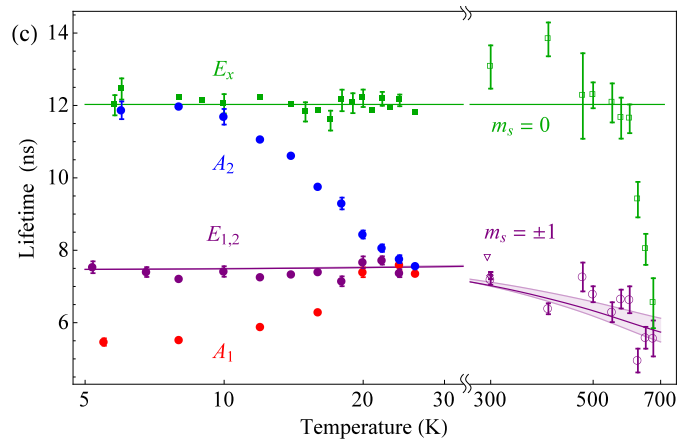

Figure 3.4: The results of the ISC model. (a) The range (blue shading) of predicted values of $\Gamma_{A_{1}}$ corresponding to the range of possible values of $\lambda_{\perp}$. The orange line is the measured value of $\Gamma_{A_{1}}$, the black region indicates our extracted value of $\Delta$ (the $\left|A_{1}\right\rangle-\left|{ }^{1} A_{1}\right\rangle$ energy spacing), and the red region indicates the values of $\Delta$ that are excluded by the measured $\Gamma_{E_{1,2}} / \Gamma_{A_{1}}$ ratio. (b) The predicted (blue) and measured (orange) $\Gamma_{E_{1,2}} / \Gamma_{A_{1}}$ ratio. Because we assume no acoustic phonon cutoff, the blue plot represents an upper bound on the ratio for a given $\Delta$. We can therefore exclude the values of $\Delta$ indicated by the red region, which is reproduced in (a). (c) The lifetimes of the $m_{s}=0$ (green) and $\left|m_{s}\right|=1$ (purple) states predicted for a range of temperatures. The data from $295 \mathrm{~K}$ to $700 \mathrm{~K}$ are taken from Refs. [100] ( $\square$ and $\bigcirc$ ), [99] $(\diamond)$, and [98] ( $\nabla)$. 
the measured and predicted values of $\Gamma_{A_{1}}$ confines $\Delta$ to two regions: around $43 \mathrm{meV}$ and from 344 to $430 \mathrm{meV}$.

We next evaluate $\Gamma_{E_{1,2}} / \Gamma_{A_{1}}$, which depends only on electron-phonon coupling parameters and the vibronic overlap function, not on the SO coupling rate. We extract $\eta=2 \pi \times(44.0 \pm 2.4) \mathrm{MHz} \mathrm{meV}^{-3}$, which parameterizes the electron-phonon coupling strength, from the $\left|E_{\mathrm{x}}\right\rangle-\left|E_{\mathrm{y}}\right\rangle$ mixing data shown in Fig. 3.2(a) (see Appendix A for details). In Fig. 3.4(b), we plot the ratios predicted by Eqs. 3.3-3.4 ${ }^{3}$ and extracted from Fig. 3.3. We assume no acoustic cutoff energy $(\Omega \rightarrow \infty)$ in order to maximize the range of acoustic phonon modes that contribute to $\Gamma_{E_{1,2}}$, making the predicted ratio an upper bound. Even so, we find that the predicted and measured ratios are inconsistent for $\Delta<148 \mathrm{meV}$, which uniquely confines $\Delta$ to the region from 344 to $430 \mathrm{meV}^{4}$.

We scale our model up to higher temperatures [104] and find that, for this range of $\Delta$, its predictions are consistent with published lifetimes of the $m_{s}=0$ and $\left|m_{s}\right|=$ $1{ }^{3} \mathrm{E}$ states at temperatures between $295 \mathrm{~K}$ and $600 \mathrm{~K}$, as shown in Fig. 3.4(c). An additional decay mechanism, which is not captured by our model of ISC decay, significantly shortens the lifetime of the $m_{s}=0$ states above $600 \mathrm{~K}$. This decay mechanism is discussed further in Ref. [104].

\footnotetext{
${ }^{3}$ The value of $\Gamma_{E_{1,2}}$ calculated in 3.4 includes a correction to Eq. 3.4 due to a second-order ISC process that uses $\left|{ }^{1} E_{1,2}\right\rangle$ as intermediate states instead of $\left|A_{1}\right\rangle$. This correction, which is described explicitly in Ref. [104], lowers $\Gamma_{E_{1,2}} / \Gamma_{A_{1}}$ by $21 \%$ at $\Delta=148 \mathrm{meV}$.

${ }^{4}$ In this region, we find that the predicted and measured ratios match for cutoff energies of 74 to $93 \mathrm{meV}$. These values, which are below the Debye energy (194 meV [114]) and close to the energies of the quasi-local phonon modes that dominate the ${ }^{3} E(64 \mathrm{meV}[110,115])$ and $\left|{ }^{1} A_{1}\right\rangle(71 \mathrm{meV}[57])$ PSBs, are physically reasonable.
} 
Chapter 3: Phonon-induced population dynamics and intersystem crossing in nitrogen-vacancy centers

\subsection{Conlusions and outlook}

We have elucidated, both experimentally and theoretically, the roles that electronphonon interactions play in NV center dynamics. Further exploration of either of the phonon roles addressed in this chapter may yield intriguing applications. Resonant electron-phonon coupling in the ${ }^{3} E$ manifold could be used to optically cool a high-Q diamond resonator $[116,117]$ close to the vibrational ground state [118]. Such efforts would complement the growing interest in using electron-phonon coupling in the ${ }^{3} A_{2}$ states to manipulate the electron spin $[119,120]$ or to generate spin-squeezed states of NV ensembles [121]. Further, our understanding of the ISC mechanism may enable efforts to engineer the ISC rate by, for example, applying a large static strain to shift the energy spacings between the spin-triplet and -singlet states [94]. Such an advance would provide an across-the-board enhancement to the spin initialization and readout techniques on which room-temperature NV center applications depend. Finally, our experimentally validated ISC model has confined the unknown energy of the ${ }^{3} E$ and $\left|{ }^{1} A_{1}\right\rangle$ states to a region that can be explored in future optical spectroscopy. 


\section{Chapter 4}

\section{Indistinguishable photons from}

\section{separated silicon-vacancy centers}

\section{in diamond}

\subsection{Introduction}

The realization of quantum networks, in which local quantum processing nodes are connected over long distances via optical photons, is an outstanding challenge in quantum information science[8]. Over the past few years, atom-like systems in the solid state have emerged as a promising platform for achieving this goal. Key building blocks have been demonstrated using nitrogen-vacancy (NV) centers in diamond, including long lived qubit memory[92], spin-photon[122] and spin-spin entanglement[65], as well as teleportation between distant stationary qubits[123]. While NV centers can be used as excellent quantum registers, the current efforts 
Chapter 4: Indistinguishable photons from separated silicon-vacancy centers in diamond

to scale up these proof-of-concept experiments are limited by the small probability of coherent photon emission from NV centers and their spectral stability[49, 54]. Here we demonstrate that silicon-vacancy $(\mathrm{SiV})$ centers in diamond can be used to efficiently generate coherent optical photons with excellent spectral stability. We show that these features are due to the inversion symmetry associated with SiV centers, and demonstrate generation of indistinguishable single photons from separate emitters in a Hong-Ou-Mandel (HOM) interference experiment [67].

The negatively charged SiV center in diamond consists of a silicon atom and a split vacancy as shown in Figure 4.1(a) $[124,59]$. The silicon atom is centered between two empty lattice sites, and this $\mathrm{D}_{3 \mathrm{~d}}$ geometry forms an inversion symmetric potential for the electronic orbitals[124]. Recent measurements[59, 58] and first principle calculations[125] have contributed to a detailed understanding of the electronic structure of the SiV center. As shown in Figure 4.1(b), the ground and excited states each consist of a fourfold degenerate manifold where two degenerate orbitals are occupied by a $S=1 / 2$ particle[126]. At zero magnetic field, the degeneracy is partially lifted by the spin-orbit interaction. Each excited state has dipole transitions to the two ground states forming an optical $\Lambda$ system, resulting in the emission spectrum shown in Figure 4.1(c). These four transitions comprise the zero-phonon line (ZPL), which contains more than $70 \%$ of the total fluorescence. Remarkably, as discussed below, the inversion symmetry results in weak coupling of the ZPL transitions to charge fluctuations in the SiV environment. This leads to the absence of spectral diffusion[127] and a narrow inhomogeneous distribution[128]. 
(a)
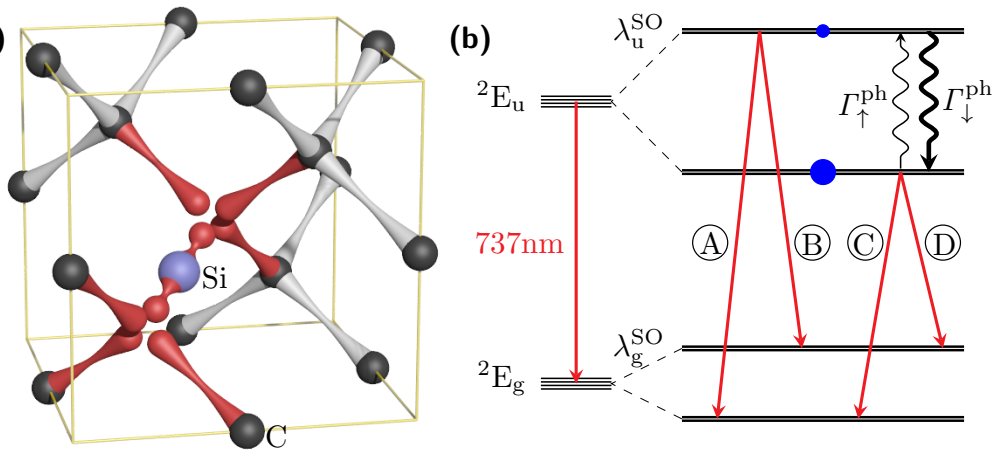

(c)

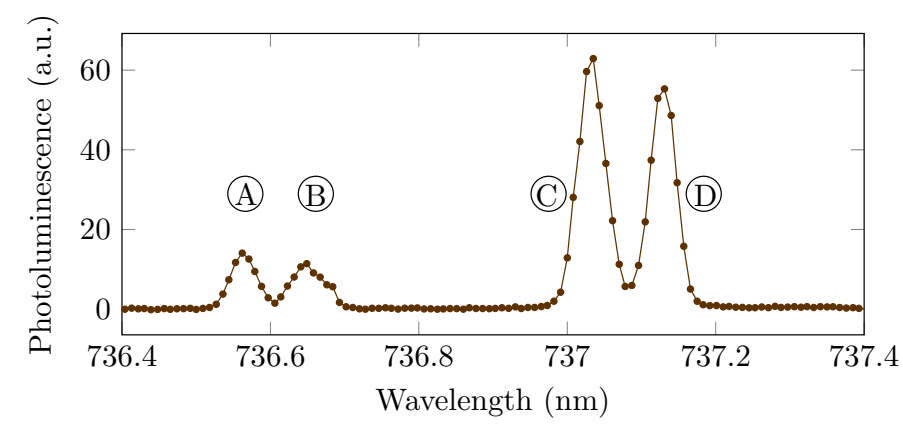

Figure 4.1: Electronic structure and optical transitions of the SiV center. (a) The center is aligned along a $\langle 111\rangle$ axis of the diamond host crystal, with the Silicon atom (Si) located in the middle of two empty lattice sites. The system has $\mathrm{D}_{3 \mathrm{~d}}$ symmetry which includes inversion symmetry. (b) The optical transition is between different parity states, ${ }^{2} \mathrm{E}_{\mathrm{u}}$ and ${ }^{2} \mathrm{E}_{\mathrm{g}}$. Spin orbit interaction $\left(\lambda_{\mathrm{so}}^{\mathrm{u}} \sim 250 \mathrm{GHz}, \lambda_{\mathrm{so}}^{\mathrm{g}} \sim 50 \mathrm{GHz}\right)$ partially lifts the degeneracy giving rise to doublets in the ground and excited states. Transitions A, B, C, D are all dipole allowed. (c) The emission spectrum measured using off-resonant excitation at $532 \mathrm{~nm}$ on a single $\mathrm{SiV}$ center at $4.5 \mathrm{~K}$. 
Chapter 4: Indistinguishable photons from separated silicon-vacancy centers in diamond

\subsection{Isolating two resonant $\mathrm{SiV}$ centers in a high density ensemble}

To demonstrate coherent emission of indistinguishable single photons from separate $\mathrm{SiV}$ centers we use a Hong-Ou-Mandel interference experiment. The interference of two identical single photons impinging on a beamsplitter results in perfect photon bunching, with a vanishing probability of detecting coincident photons at the two different output ports. In our experiments two separate SiV centers, cooled to cryogenic temperatures, were excited using a two-channel confocal optical microscope shown in Figure 4.2(a). Dichroic mirrors were used to simultaneously collect the SiV fluorescence on both the ZPL $(\lambda \sim 737 \mathrm{~nm})$ and phonon-side-band (PSB, $\lambda \sim 760-860 \mathrm{~nm})$. In order to isolate a single two-level transition, the emission spectrum was filtered by solid etalons (Figure 2(b)) with a free spectral range of $20 \mathrm{GHz}$ and a bandwidth of $1 \mathrm{GHz}$. The etalons were tuned by temperature to transition $\mathrm{C}$ and the transmitted fluorescence spectrum is shown in Figure 4.2(c), where only a single peak is visible as desired for indistinguishable photon generation.

To probe the inhomogeneous distribution within the sample and select spectrally overlapping sites, the emitters were resonantly excited with a $737 \mathrm{~nm}$ probe laser using the ZPL. The laser was tuned to the center frequency $\left(\nu_{0}\right)$ of the inhomogeneous distribution for transition $\mathrm{C}$ while monitoring fluorescence intensity in the PSB. Figure 4.3(a) shows the diamond sample imaged by this technique in a region where the resonant site density was high, leading to a high background in any photon correlation experiments. In order to isolate single $\mathrm{SiV}$ centers and minimize background 

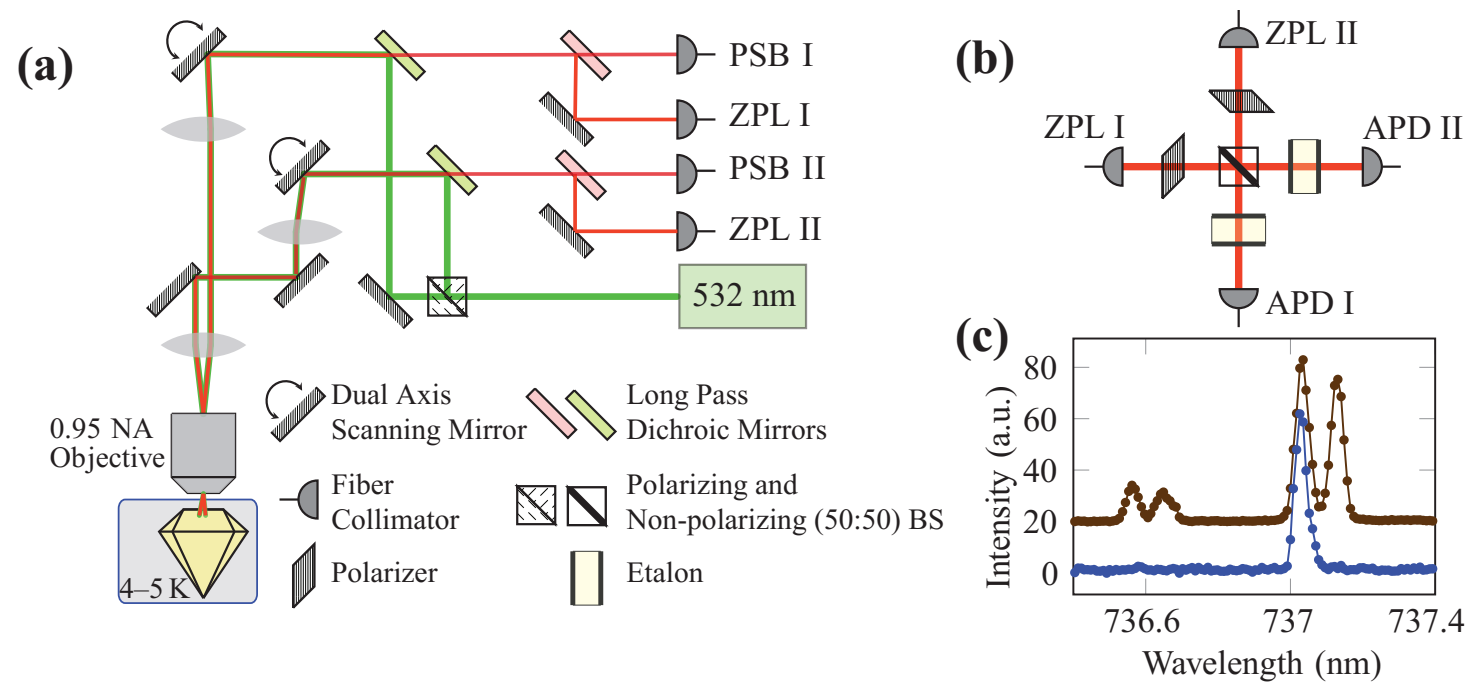

Figure 4.2: Schematic of the two-channel confocal microscope built for the HOM experiment. (a) Channels I and II were used to address different emitters separated by tens of micrometers in the same sample. A continuous-wave $532 \mathrm{~nm}$ laser was used for excitation, and fluorescence was collected in single mode fibers on ZPL and PSB ports simultaneously. (b) Collected ZPL fluorescence from the two channels were directed onto a free-space 50:50 non-polarizing beam splitter. Linear polarizers were used to control the polarization of the single photons varying their distinguishability. Etalons were used to filter transition $\mathrm{C}$ before detection. (c) Emission spectrum before (brown) and after the etalons (blue). 
Chapter 4: Indistinguishable photons from separated silicon-vacancy centers in diamond

from other emitters[32], the laser was tuned to the edge of the inhomogeneous distribution $\left(\nu_{1}\right)$ in Figure 4.3(b). Figures 4.3(c,d) show the two emitters that were chosen for the HOM experiment at frequency $\nu \sim \nu_{1}$. The images in Figures 4.3(c,d) were taken under $532 \mathrm{~nm}$ excitation while detecting ZPL photons through the etalons. Here the frequency selectivity is limited by the bandwidth of the etalons $(\sim 1 \mathrm{GHz})$ and therefore more emitters are visible than in the resonant excitation scan (Figure 4.3(b)). Photoluminescence excitation (PLE) spectra of the emitters, $\mathrm{SiV}_{\mathrm{I}}$ (green) and $\mathrm{SiV}_{\mathrm{II}}$ (pink), reveal transitions separated by $52.1 \mathrm{MHz}$ with full width half maximum (FWHM) of 136 and $135 \mathrm{MHz}$ respectively. For comparison, the lifetime of the excited states was measured to be $1.73 \pm 0.05 \mathrm{~ns}$ at temperatures below $50 \mathrm{~K}$ corresponding to a transform limited linewidth of $94 \mathrm{MHz}$.

\subsection{Demonstration of HOM interference from two $\mathrm{SiV}$ centers}

For the HOM measurement, single photons emitted from $\mathrm{SiV}_{\mathrm{I}}$ and $\mathrm{SiV}_{\mathrm{II}}$ on transition $\mathrm{C}$ were directed to the input ports 1 and 2 of the beamsplitter respectively (see Figure 4.2(a,b)). Figure 4.4 shows two measurements where the degree of indistinguishability of single photons is varied by changing the photon polarization. The two datasets show the second order intensity correlation function, $g^{2}(\tau)$, measured for indistinguishable (pink) and distinguishable (green) photon states. For identically polarized indistinguishable photons, we find $g_{\|}^{2}(0)=0.26 \pm 0.05$ where the error bars denote shot noise estimates. After rotating the fluorescence polarization of $\mathrm{SiV}_{\text {II }}$ by 


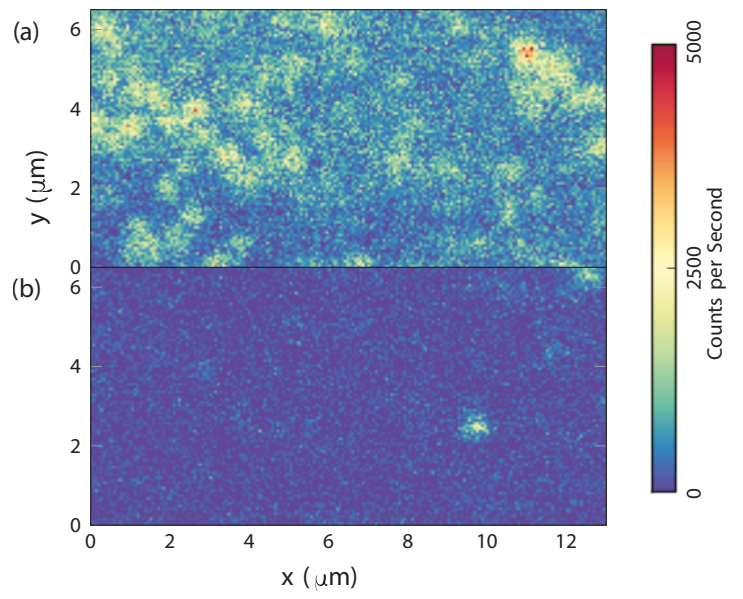

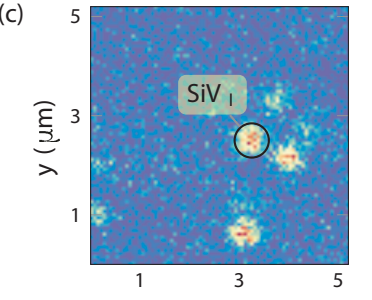
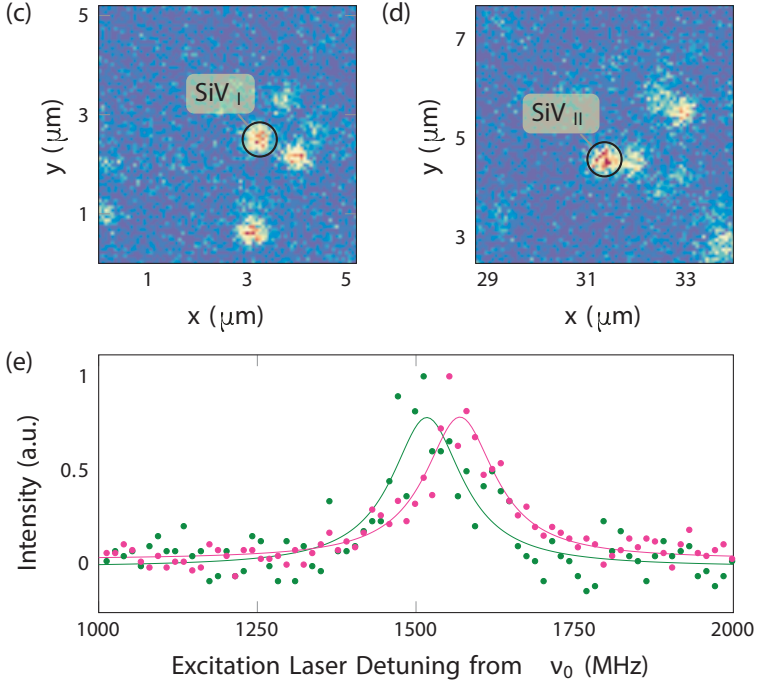

Figure 4.3: Inhomogeneous distribution of SiV centers. (a) The probe laser frequency was fixed to the ensemble average of $\nu_{0}=406.7001 \mathrm{THz}$ for transition $\mathrm{C}$ while scanning the sample. A high density of resonant emitters is visible with a large background. The color bar applies to (a-d). (b) Scan of the same region with the laser tuned to $\nu_{1}=\nu_{0}+1.5 \mathrm{GHz}$. Due to the narrow inhomogeneous distribution, only few resonant sites are visible and the background level is low. (c) and (d) show the two emitters, $\mathrm{SiV}_{\mathrm{I}}$ and $\mathrm{SiV}_{\mathrm{II}}$, used for the HOM interference experiment at frequency $\nu \sim \nu_{1}$. (e) PLE spectrum for $\mathrm{SiV}_{\mathrm{I}}$ (green) and $\mathrm{SiV}_{\mathrm{II}}$ (pink) with measured full width half maximum (FWHM) of 135.8 and 134.6 MHz respectively, and lines separated by $52.1 \mathrm{MHz}$. 
Chapter 4: Indistinguishable photons from separated silicon-vacancy centers in diamond

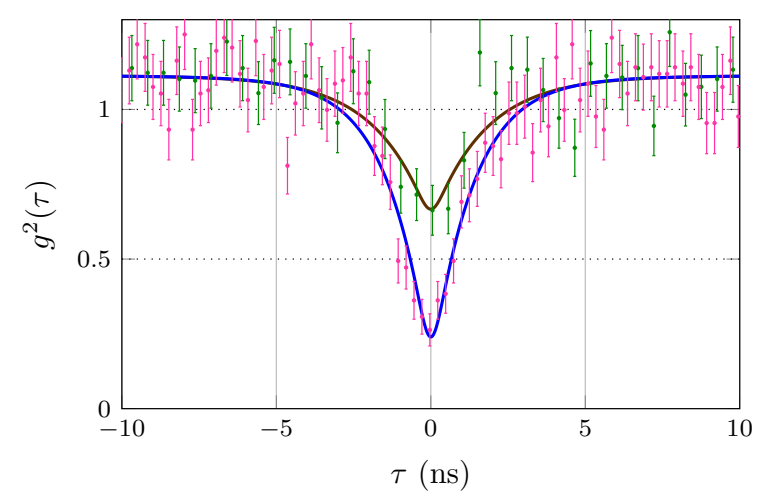

Figure 4.4: Hong-Ou-Mandel interference experiment. The second order intensity correlation function $g^{2}(\tau)$ is plotted for two cases: (i) Pink data shows the results for indistinguishable single photons with identical polarizations, $g_{\|}^{2}(0)=0.26 \pm 0.05$ - The error bars denote shot noise estimates. (ii) Green data shows the results when photons from one emitter are orthogonally polarized and hence distinguishable, $g_{\perp}^{2}(0)=0.66 \pm 0.08$. The blue and brown solid lines represent our model using independently measured parameters, only fitting a single parameter for background events in both datasets.

$90^{\circ}$ to make the photon sources distinguishable, $g_{\perp}^{2}(0)=0.66 \pm 0.08$ was observed. These results clearly demonstrate two-photon interference corresponding to a measured HOM visibility of

$$
\eta=\frac{g_{\perp}^{2}(0)}{g_{\|}^{2}(0)+g_{\perp}^{2}(0)}=0.72 \pm 0.05
$$

The time dynamics of $g^{2}(\tau)$ is understood via independent measurements of the excited state lifetime, absorption linewidth, and detector timing response. Our model (solid curves, see Appendix B) is in excellent agreement with the measured time dynamics, showing that the emitters were spectrally stable throughout the 4-hour acquisition period. We find that the interference visibility, $\eta$, is limited by about equal contributions from detector timing response and background events. 


\subsection{Discussion and outlook}

We next turn to a discussion of the key properties of SiV centers which made the present observations possible. Despite uncertainty about the absolute quantum yield[127], the strong ZPL of SiV [55] means that photons are emitted at high rates into the optical transition of interest. Inhomogeneous broadening corresponded to only a few transition linewidths (see Appendix B), and high spectral stability of the transitions has been observed in bulk diamond[127] and nanodiamonds[126]. Together with these observations, our work shows that the optical coherence properties of $\mathrm{SiV}$ centers can be superior to those of NV centers[64, 54]. Some of this advantage can be understood to result from the inversion symmetry of SiV centers (which reduces sensitivity to electric field). In addition, it is important to consider the effects of phonons (strain) resulting in homogenous (inhomogeneous) broadening mechanisms.

The electronic orbitals of the $\mathrm{SiV}$ center are parity eigenstates due to the inversion symmetry of the defect. The optical transitions take place between states of different parity, ${ }^{2} \mathrm{E}_{\mathrm{g}}$ and ${ }^{2} \mathrm{E}_{\mathrm{u}}$, which differ in phase but have similar charge densities[125]. This small change in the electronic charge density results in the strong ZPL, since optical excitations do not couple efficiently to local vibrations. The coherence of the optical transitions can also suffer from spectral diffusion, a time dependent change in the optical transition frequencies that results in an increased linewidth. This effect is commonly observed for NV centers, where the dominant source of spectral diffusion has been shown to be from local electronic charge fluctuations[129]. These changes in the charge environment result in a fluctuating electric field at the emitter which reduces the coherence of the optical transitions via DC Stark shift[48, 54]. The sen- 
Chapter 4: Indistinguishable photons from separated silicon-vacancy centers in diamond

sitivity of the optical transition frequencies to electric field fluctuations depends on the permanent electric dipole moments of the orbital states of the emitter. Since the electronic states of the $\mathrm{SiV}$ center have vanishing permanent electric dipole moments due to their inversion symmetry, the optical transitions are relatively insensitive to external electric fields. This protects the optical coherence from charge dynamics in the crystal, preventing spectral diffusion and narrowing the inhomogeneous distribution of transition frequencies.

Additional homogeneous and inhomogeneous broadening mechanisms are provided by phonons and strain. Displacements of atoms in the host crystal can affect the optical transitions in two different ways. Static distortions, or strain, may reduce the symmetry of the defect and change the energy splittings[128] shown in Figure 4.1(b). A variation in local strain contributes to the inhomogeneous distribution of the resonance frequencies. Displacements of the atoms can also give rise to dynamic effects during an optical excitation cycle. Acoustic phonons have been shown to cause orbital relaxation between $\left|E_{x}\right\rangle$ and $\left|E_{y}\right\rangle$ states for the NV center in diamond[85]. For $\mathrm{SiV}$ centers, a similar process can happen between excited state orbitals by absorption $\left(\Gamma_{\uparrow}^{\mathrm{ph}}\right)$ or emission $\left(\Gamma_{\downarrow}^{\mathrm{ph}}\right)$ of an acoustic phonon as shown in Figure 4.1(b). Populations in the upper and lower excited state branches follow a Boltzmann distribution confirming thermalization of orbital states by phonons[128, 127]. At low temperatures $\left(k_{\mathrm{B}} T \ll \hbar \lambda_{\mathrm{so}}^{\mathrm{u}} \sim 250 \mathrm{GHz}\right)$ spontaneous emission dominates over stimulated processes $\left(\Gamma_{\uparrow}^{\mathrm{ph}} \ll \Gamma_{\downarrow}^{\mathrm{ph}}\right)$. To obtain an optical transition isolated from the phonon bath, our experiments were performed at $4.5-5 \mathrm{~K}(\sim 100 \mathrm{GHz})$ using the lower excited state branch. At these temperatures, we estimate a thermal broadening on transition $\mathrm{C}$ of 
Chapter 4: Indistinguishable photons from separated silicon-vacancy centers in diamond

about $12 \mathrm{MHz}[127]$.

Our observations establish the SiV center as an excellent source of indistinguishable single photons. A strong ZPL transition, narrow inhomogeneous distribution, and spectral stability combine to make it a promising platform for applications in the fields of quantum networks and long distance quantum communication. In particular, it should be possible to integrate SiV centers inside nanophotonic cavities[130, 52, 131, 132, 49] while maintaining their spectral properties owing to their insensitivity to electric fields. This may allow the realization of $\mathrm{GHz}$ bandwidth deterministic single photon sources[133] and a broadband system for quantum nonlinear optics at the single photon level[134]. The small inhomogeneous distribution also makes SiV centers promising candidates as sources of multiple indistinguishable photons for linear optics quantum computing[135]. Furthermore, the spin degree of freedom in the ground state [126] can potentially be utilized to store quantum information, allowing the use of SiV centers as quantum registers for quantum network applications [136]. Coupling to the ${ }^{29} \mathrm{Si}$ nuclear spin via hyperfine interactions[137] might allow realization of long lived quantum memories[92]. Beyond these specific applications, the symmetry arguments presented above suggest that inversion symmetry might play an important role in the identification of new centers with suitable properties for quantum information science and technology[138]. 


\section{Chapter 5}

\section{Electron-phonon processes of the}

\section{silicon-vacancy center in diamond}

\section{$5.1 \quad$ Introduction}

Colour centres in diamond have emerged as attractive systems for applications in quantum metrology, quantum communication, and quantum information processing [139, 123, 140]. Diamond has a large band gap which allows for optical control, and it can be synthesised with high purity enabling long coherence times as was demonstrated for nitrogen-vacancy (NV) spin qubits [43]. Among many colour centres in diamond $[141,56]$, the negatively charged silicon-vacancy (SiV) centre stands out due to its desirable optical properties. In particular, near transform limited photons can be created with high efficiency due to the strong zero-phonon line (ZPL) emission that constitutes $\sim 70 \%$ of the total emission. SiV centres can also be created with a narrow inhomogeneous distribution that is comparable to the transform limited 
optical linewidth [127]. These optical properties, due to the inversion symmetry of the system which suppresses effects of spectral diffusion, recently enabled demonstration of two-photon interference from separated emitters [142] that is a key requirement for many quantum information processing protocols $[136,143,144,145]$.

Interfacing coherent optical transitions with long-lived spin qubits is a key challenge for quantum optics with solid state emitters [122, 146, 147, 54, 49]. This challenge may be addressed using optically accessible electronic spins in SiV centres [126]. It has recently been demonstrated that coherent spin states can be prepared and read out optically [61,62], although the spin coherence time was found to be limited by phonon-induced relaxation in the ground states [61]. In this Chapter, we present the first systematic study of the electron-phonon interactions that are responsible for relaxation within the ground and excited states of the $\mathrm{SiV}$ centre. This is achieved by measuring the temperature dependence of numerous processes within the centre. A comprehensive microscopic model is then developed to account for the observations. In Section 5.4.1 we discuss the implications of these phonon processes for spin coherences in the SiV ground state, and identify approaches that could extend the spin coherences.

The SiV centre consists of an interstitial silicon atom in a split-vacancy configuration with $\mathrm{D}_{3 \mathrm{~d}}$ symmetry as illustrated in Fig.5.1(a) [124]. This symmetry gives rise to an electronic level structure consisting of ground $\left({ }^{2} \mathrm{E}_{\mathrm{g}}\right)$ and excited $\left({ }^{2} \mathrm{E}_{\mathrm{u}}\right)$ states that both have E symmetry and double orbital degeneracy. The degenerate orbital states are occupied by a single hole with $S=1 / 2[148,59,58,125]$, leading to both orbital and spin degrees of freedom. In the absence of off-axis strain or magnetic 
fields, the spin-orbit interaction $\left(\sim \lambda_{\mathrm{so}}^{\mathrm{g}, \mathrm{u}} S_{z} L_{z}\right)$ determines the eigenstates with well defined orbital and spin angular momentum [58]. Optical and phononic transitions between these eigenstates couple only to the orbital degree of freedom and are spin conserving. We therefore focus on the orbital dynamics within the ground and excited states, which can each be described as an effective two-level system consisting of two orbital states $\left\{\left|L_{z}= \pm 1\right\rangle=\left|e_{ \pm}^{g, u}\right\rangle\right\}$ for a given spin projection as shown in Fig. 5.1(b)[58]. Phonons can introduce vibronic coupling between $\left|e_{+}\right\rangle$and $\left|e_{-}\right\rangle$orbitals, resulting in population transfer between orbitals at rates $\gamma_{+,-}^{g, u}[149,150]$. This effect, also called the dynamic Jahn-Teller effect, has been observed in the excited states of the NVcentre $[85,151]$ where a similar orbital degeneracy is present.

\subsection{Experimental results}

\subsubsection{Excited states}

The spin-orbit interaction results in four optical dipole transitions, labelled A$\mathrm{D}$ in order of increasing wavelength, centred around $\sim 737 \mathrm{~nm}$ at cryogenic temperatures $[128,152,55]$. At liquid helium temperatures, the optical linewidths are broader for transitions A and B than for the lower energy transitions C and D [127]. This was attributed to thermal relaxation reducing the effective lifetime of the upper branch via the decay rate $\gamma_{-}^{\mathrm{u}}$, which is faster than $\gamma_{+}^{\mathrm{u}}$ by the Boltzmann factor $\gamma_{-}^{\mathrm{u}}=\gamma_{+}^{\mathrm{u}} \exp \left(\lambda_{\mathrm{so}}^{\mathrm{u}} / k_{\mathrm{B}} T\right)[128,152]$.

To probe the microscopic mechanism of the orbital relaxation in the excited states, the temperature dependence of the linewidth of transition $\mathrm{D}$ was measured for individ- 
(a)

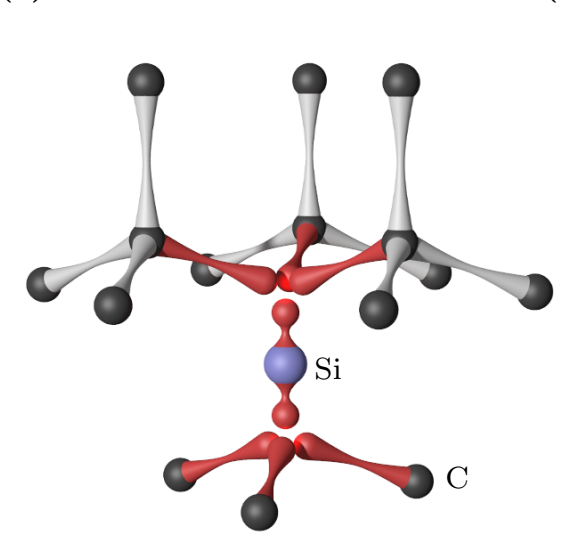

(b)

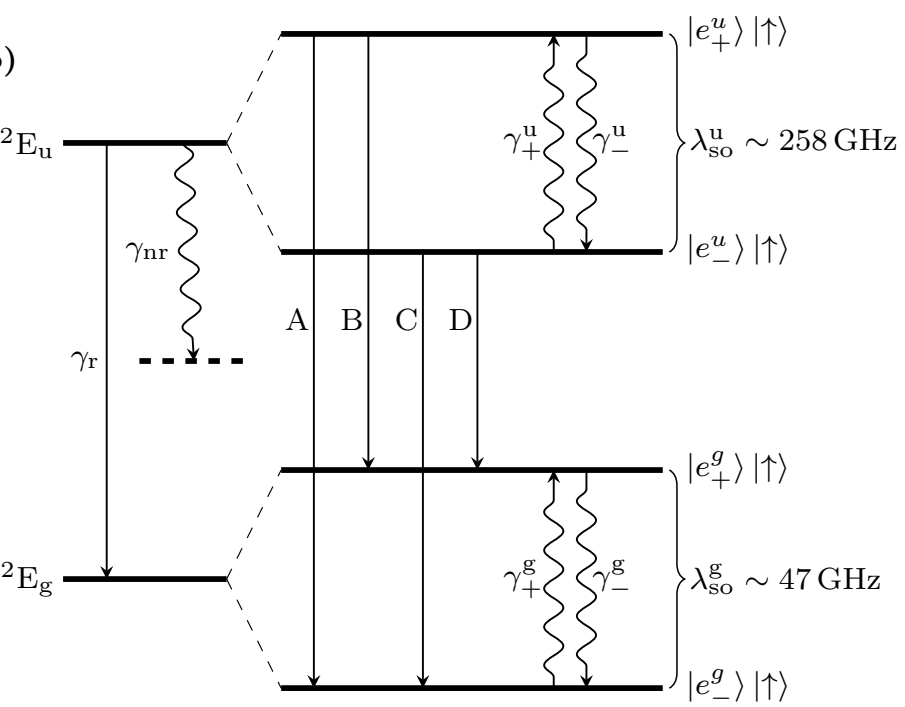

Figure 5.1: Molecular structure and electronic dynamics of SiV. (a) The SiV centre consists of a silicon atom centred between two neighbouring vacant lattice sites. (b) The optical transitions are between states of E symmetry with opposite parity $\left({ }^{2} \mathrm{E}_{\mathrm{g}},{ }^{2} \mathrm{E}_{\mathrm{u}}\right) \cdot \gamma_{\mathrm{r}}\left(\gamma_{\mathrm{nr}}\right)$ are radiative (non-radiative) decay rates out of the excited states. Straight (curved) lines denote the radiative (non-radiative) transitions. In both the ground and excited states, the four-fold degeneracy is partially lifted by the spinorbit interaction $\lambda_{\text {so }}^{\mathrm{g}, \mathrm{u}}[59,58]$. Every level illustrated here is a spin- $1 / 2$ doublet (e.g. $\left\{\left|e_{-}\right\rangle|\uparrow\rangle,\left|e_{+}\right\rangle|\downarrow\rangle\right\}$ for the lowest energy level), and for clarity only the spin-up levels are drawn. Implications of this study for the spin sublevels are discussed in Section 5.4.1. The horizontal dashed line denotes the unidentified level (either an additional electronic level or excited vibrational state of ${ }^{2} \mathrm{E}_{\mathrm{g}}$ ) involved in the non-radiative decay between the ground and excited states.

ual SiV centres. Since these SiV centres exhibit negligible spectral diffusion [127], the measured optical linewidths $\Gamma(T)$ correspond to homogeneous broadening mechanisms associated with depolarisation and dephasing: $\Gamma(T)=\gamma_{\mathrm{r}}+\gamma_{\mathrm{nr}}(T)+\gamma_{+}^{\mathrm{u}}(T)+\gamma_{d}(T)$. The non-radiative decay rate $\gamma_{\mathrm{nr}}(T)$ has a very weak temperature dependence, as discussed later in Section 5.2.3, leading to a small contribution compared to the other rates for all temperature regions of interest. The most significant temperature dependence comes from the relaxation rates within the excited states: $\gamma_{+}^{\mathrm{u}}(T)$ and $\gamma_{d}(T)$. 
As will be shown in Section 5.3.2, the optical transition linewidth is dominated by relaxation rates in the excited states with little contribution from the ground states.

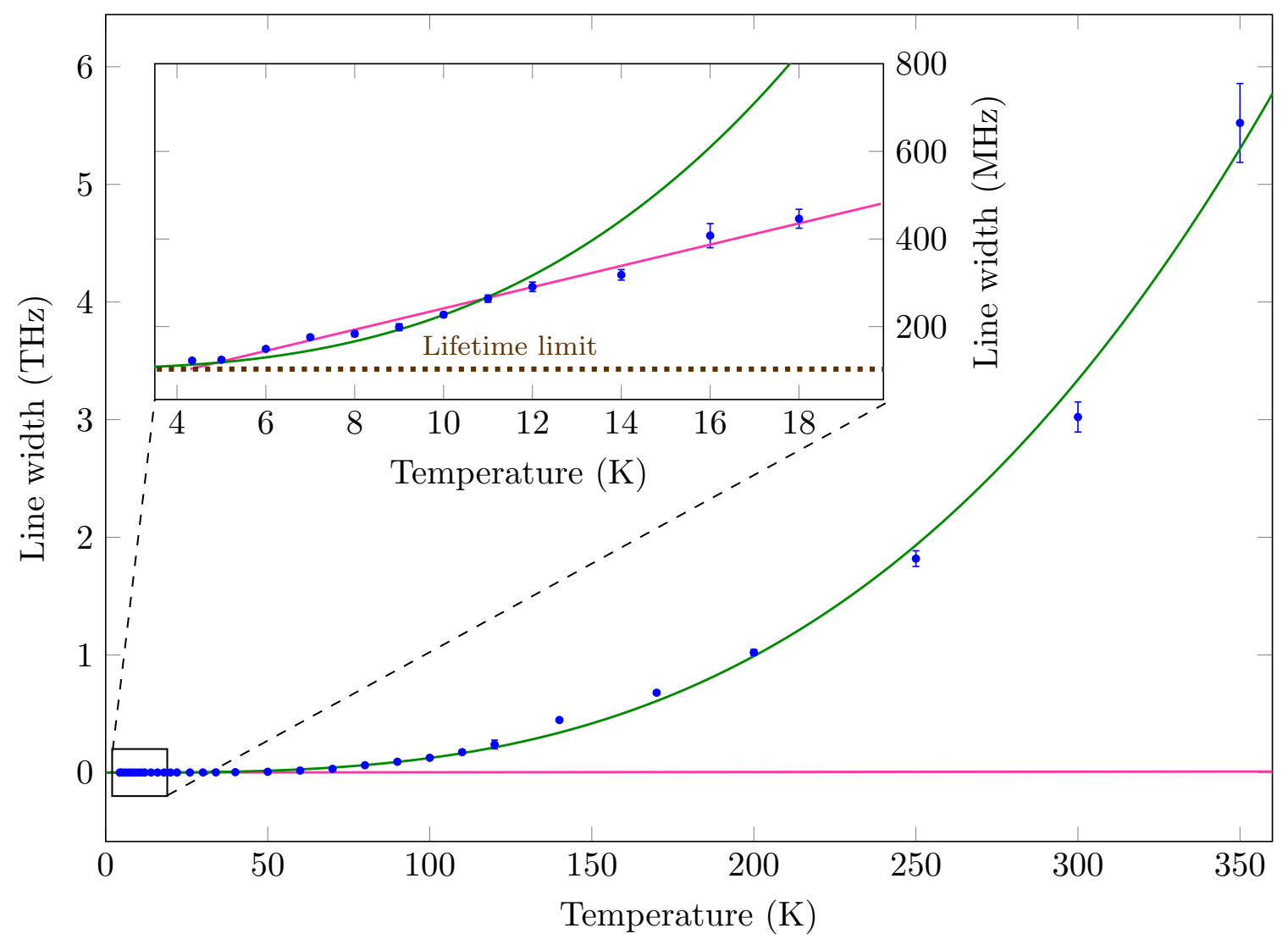

Figure 5.2: Linewidth of transition D measured for different temperatures. Each linewidth was determined by Lorentzian fits for multiple sites. The green fit corresponds to a cubic scaling over the high temperature range $(>70 \mathrm{~K})$ after the spectrum has merged to two peaks and one peak at $>120 \mathrm{~K}$. At low temperatures $(<20 \mathrm{~K})$ the pink fit represents a linear scaling seen in the inset. For the temperature range in between these two regimes the scaling of the linewidth crosses over from linear to cubic behaviour.

For temperatures between $4 \mathrm{~K}$ to $50 \mathrm{~K}$, the optical transition linewidth was measured using photoluminescence excitation in a continuous flow cryostat, where a weak probe laser was scanned across transition D and fluorescence in the phonon-sideband 
(PSB) was detected. At higher temperatures, $532 \mathrm{~nm}$ excitation was used and emission linewidths were measured with a spectrometer (Princeton Instruments Acton 2500 equipped with a Pixis 100 cooled CCD-array and a $1596 \mathrm{~mm}^{-1}$ grating) giving a resolution of $16 \mathrm{GHz}$. To measure fundamental properties of the $\mathrm{SiV}$ centre, a bulk diamond sample containing highly uniform defect sites and low strain was used in these experiments. It was a low strain HPHT type-IIa diamond with a $\{100\}$ surface on which a $60 \mu \mathrm{m}$ layer incorporating $\mathrm{SiV}$ was created by microwave-plasma chemical-vapour-deposition (MPCVD). This sample was used in previous publications $[59,127,142]$ and shows a narrow inhomogeneous distribution for the $\mathrm{SiV}$ optical transitions.

Fig. 5.2 shows the full width at half maximum (FWHM) linewidths (determined from Lorentzian fits) measured for single $\mathrm{SiV}$ sites in a $200 \times 200 \mathrm{\mu m}^{2}$ region containing ${ }^{28} \mathrm{Si}$. Above $\sim 70 \mathrm{~K}$ the linewidth scales as the cube of the temperature $(\Gamma=(103+$ $\left.\left.0.12 \cdot(T / K)^{3}\right) \mathrm{MHz}\right)$. However for low temperatures $(<20 \mathrm{~K})$, the behaviour deviates from $T^{3}$ and is better approximated by a linear dependence on temperature $(\Gamma=$ $(-1.05+24.26 \cdot(T / \mathrm{K})) \mathrm{MHz})$ saturating at about $4 \mathrm{~K}$ to the lifetime limited linewidth. Early studies on nanodiamonds have measured the $T^{3}$ dependence of the linewidth on temperature, but were not able to resolve this linear contribution due to a combination of inhomogeneous broadening and spectral resolution limits [153]. It is shown in Section 5.3 that the observed $T$ and $T^{3}$ mechanisms result from first- and secondorder transitions due to linear electron-phonon interactions with E-symmetric phonon modes. 


\subsubsection{Ground states}

Relaxation within the ground state doublet, $\gamma_{+}^{\mathrm{g}}$ in Fig. 5.1 (b), was probed directly using pulsed excitation and time-resolved fluorescence measurements. Transitions C and D form an optical $\Lambda$-system which allows ground state populations to be optically pumped. For these experiments a second diamond sample was used in which the properties and orientation of the $\mathrm{SiV}$ centres were known from earlier studies [59]. The sample is a low strain high-pressure high-temperature (HPHT) diamond observed through a $\{111\}$ surface with a low density of in-grown $\mathrm{SiV}$ centres and the optical properties of the SiV centres found within are comparable to those in the $\{100\}$ sample. A laser was tuned to transition D and 80 ns pulses were generated using an electro-optical amplitude modulator with a measured extinction ratio of up to $20 \mathrm{~dB}$. The signal was detected by counting the photon arrival times in relation to the laser pulses using a time-tagged data acquisition card (FAST ComTec MCS26A) giving a time resolution of up to $200 \mathrm{ps}$.

At the start of each laser pulse we observed a fluorescence peak that decayed to a steady state level. For the first laser pulse, the peak height $a$ corresponds to the thermal population in the bright state $\left(\left|e_{+}^{g}\right\rangle|\uparrow\rangle\right.$ or $\left.\left|e_{-}^{g}\right\rangle|\downarrow\rangle\right)$ which is $\sim 50 \%$ for the temperatures in our measurements. The decay of this initial peak when the laser is on corresponds to optical pumping into the dark state $\left(\left|e_{-}^{g}\right\rangle|\uparrow\rangle\right.$ or $\left.\left|e_{+}^{g}\right\rangle|\downarrow\rangle\right)$. After a dark interval $\tau$, the dark state relaxes back to the bright state, leading to a recovery of peak height $h$ for subsequent pulses. The peak height, $h(\tau)$, exhibits a simple exponential recovery indicating a single characteristic relaxation time, $T_{1}$, as shown in Fig. 5.3(a,b). This measurement was repeated for a single SiV centre at 
(a)

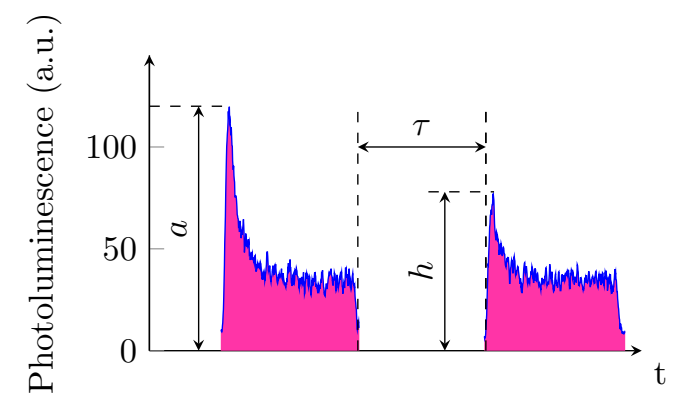

(c)

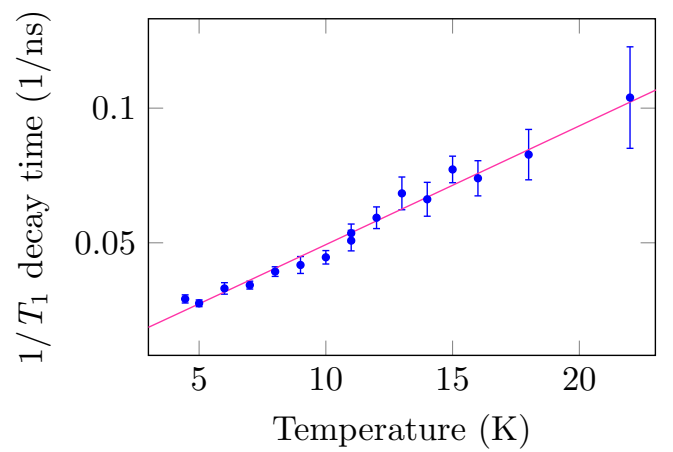

(b)

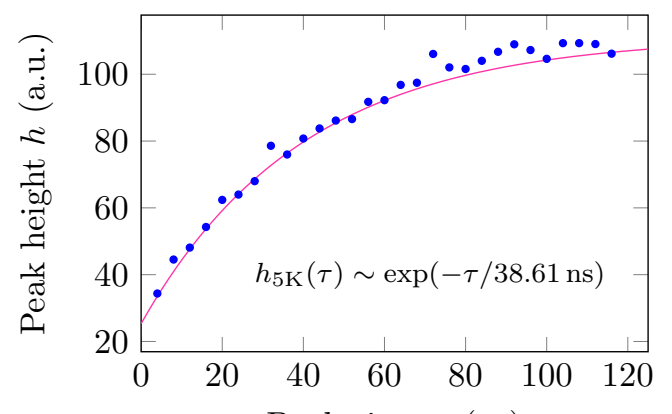

(d)

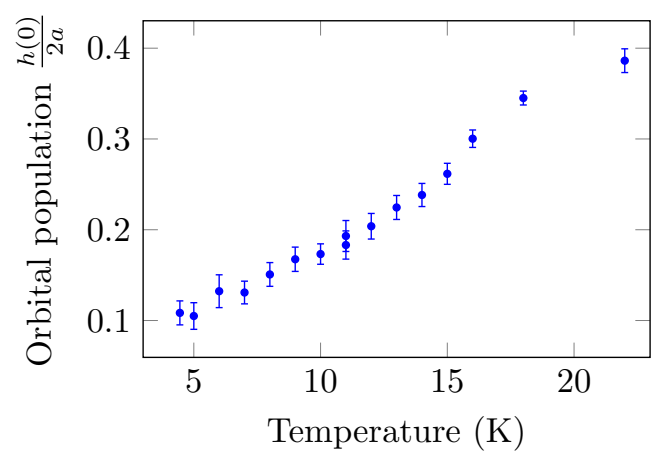

Figure 5.3: Ground state orbital relaxation time $\left(T_{1}\right)$ measurements. (a) The pulse sequence used to measure the $T_{1}$ of the ground states. A single laser was amplitude modulated to pump (first pulse) and probe (second pulse) transition D at each temperature. Photoluminescence (PL) intensity corresponds to the bright state population. (b) The height $h$ of the leading edge peak plotted for different wait times $\tau$ between the pulses. An exponential fit to the recovery of the height gives the orbital ground state relaxation time $T_{1}$. (c) Measured orbital relaxation rate, $\gamma_{+}^{\mathrm{g}}$, as a function of temperature. A fit (pink) to a single-phonon relaxation model (Section 5.3.2) shows good agreement with the data by introducing an offset on the temperature $\left(1 / T_{1}=0.0099 \cdot n(\Delta, T-2.26 \mathrm{~K})\right)$. (d) Bright state population, $h(0) / 2 a$, after optical pumping shown for different temperatures. 
various temperatures between $4.5 \mathrm{~K}$ and $22 \mathrm{~K}$ and the relaxation rate was found to scale linearly with temperature (Fig. 5.3)(c). The longest $T_{1}$ time was measured at the lowest temperatures to be $T_{1}(5 \mathrm{~K})=39 \pm 1 \mathrm{~ns}$.

We note that the steady state fluorescence level under laser excitation, $h(0)$, is determined by a competition between the optical pumping rate and thermalization rates $\left(1 / T_{1}\right)$. With increasing temperature, the thermalization rate increases (Fig. 5.3(c)) while the optical pumping rate remains nearly constant at saturation. This leads to a reduced measured peak contrast $(h(0) / 2 a$, Fig. 5.3(d)) with increasing temperature showing that the ground state of the $\mathrm{SiV}$ centre cannot be polarised at elevated temperatures.

\subsubsection{Excited state lifetimes}

The results presented so far have only highlighted the processes within the ground and excited state doublets. The transition rates from excited to ground states also have a temperature dependence which can be probed by measuring the fluorescence lifetime of the excited state as a function of temperature. Previous experiments have reported excited state lifetimes in the $\sim 1$ ns to 4 ns range along with various estimates of the quantum yield $[128,55,153,132,154,127]$. Fig. 5.1(b) shows potential radiative $\left(\gamma_{\mathrm{r}}\right)$ and non-radiative $\left(\gamma_{\mathrm{nr}}\right)$ processes taking place at the optical energy scale that determine the excited state lifetimes and the quantum yield. The total decay rate from the excited states, $\gamma_{t}(T)=1 / \tau_{0}(T)=\gamma_{\mathrm{r}}+\gamma_{\mathrm{nr}}(T)$, is a combination of a constant radiative $\left(\gamma_{\mathrm{r}}\right)$ and a temperature dependent non-radiative rate $\left(\gamma_{\mathrm{nr}}(T)\right)$.

In an attempt to identify the non-radiative process, we measured the lifetime 
of the ${ }^{2} \mathrm{E}_{\mathrm{u}}$ excited states as a function of temperature from $5 \mathrm{~K}$ to $350 \mathrm{~K}$. At each temperature, 10 separate single $\mathrm{SiV}$ centres were excited using a pulsed $532 \mathrm{~nm}$ laser with a $80 \mathrm{MHz}$ repetition rate and the measured time traces were fitted using a single exponential decay. The measured temperature dependence of $\tau_{0}(T)=1 / \gamma_{t}(T)$ is shown in Fig. 5.4(a). The excited state lifetime was found to increase as temperature was decreased down to $50 \mathrm{~K}$, where it saturated to a constant level. These results suggest there is a finite non-radiative rate $\gamma_{\mathrm{nr}}$ at room temperature, while the saturation below $50 \mathrm{~K}$ does not necessarily imply $\gamma_{\mathrm{nr}}(T<50 \mathrm{~K})=0$ as there might still be a finite spontaneous non-radiative rate at zero temperature. The observed temperature dependence in Fig. 5.4(a) can be described by the Mott-Seitz model for non-radiative relaxation, $\tau_{0}(T)=\tau_{0}(T=0 \mathrm{~K})\left(1+\alpha e^{-\frac{\Delta E}{k_{B} T}}\right)^{-1}$, with an activation energy of $\Delta E=55 \pm 2 \mathrm{meV}$ and $\alpha=3.3 \pm 0.3$ [155]. Our measurements do not, however, provide enough information to distinguish whether the system decays from ${ }^{2} \mathrm{E}_{\mathrm{u}}$ directly to a higher vibrational state of ${ }^{2} \mathrm{E}_{\mathrm{g}}$, or to an unidentified electronic level closer to ${ }^{2} \mathrm{E}_{\mathrm{u}}$ in energy. Whilst there exists some ab initio [125] and experimental [156] evidence of an additional electronic level below the excited ${ }^{2} \mathrm{E}_{\mathrm{u}}$ level, this evidence conflicts with the simple molecular orbital model of the centres electronic structure $[124,148,59,58]$, which predicts no such additional level. Future studies involving spectroscopy of the ${ }^{2} \mathrm{E}_{\mathrm{g}} \rightarrow{ }^{2} \mathrm{E}_{\mathrm{u}}$ absorption PSB and single-shot readout capability of $\mathrm{SiV}$ electronic states might help identify the relaxation paths from the ${ }^{2} \mathrm{E}_{\mathrm{u}}$ and dark states of $\mathrm{SiV}$ centres [157]. 
(a)

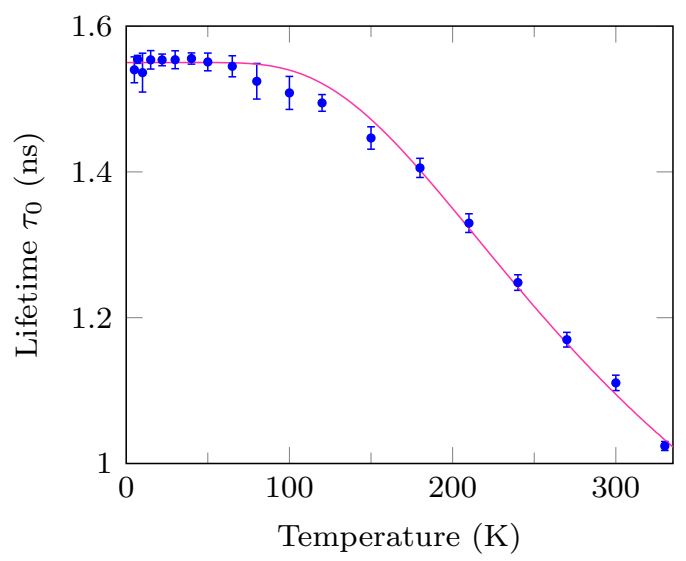

(c)

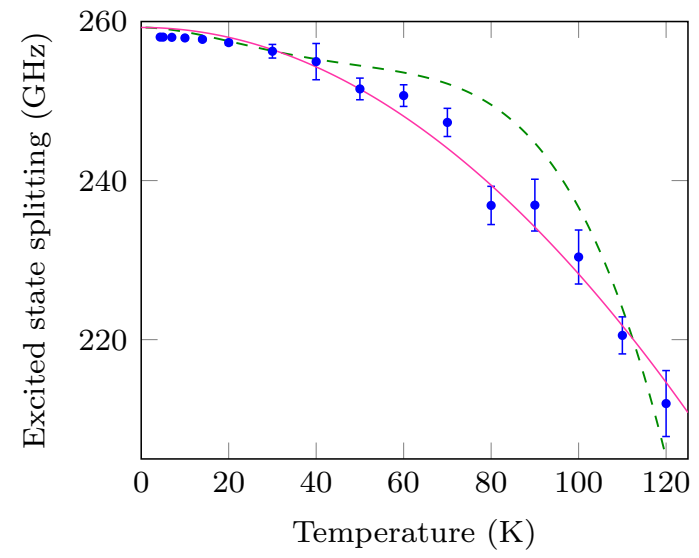

(b)

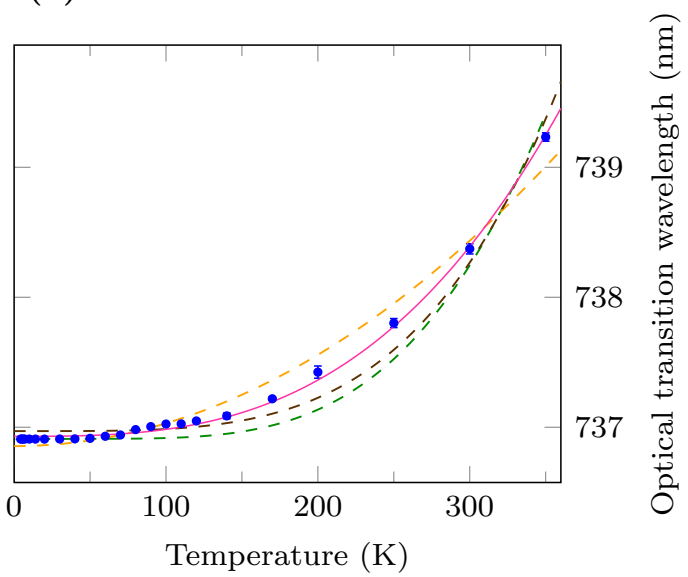

(d)

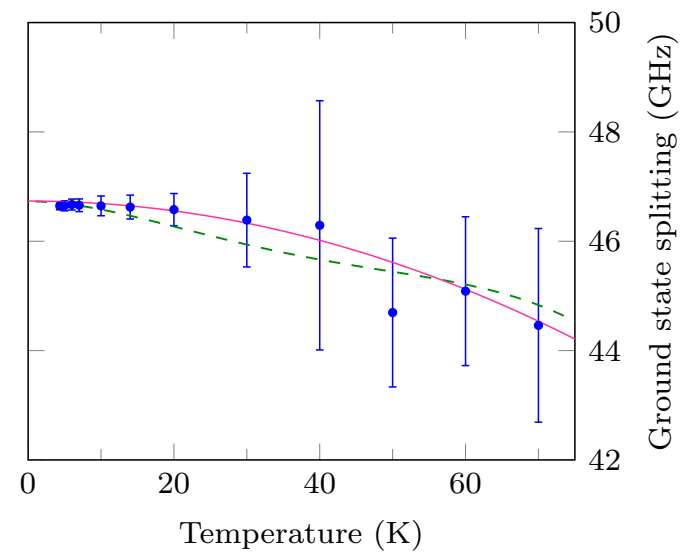

Figure 5.4: (a) Fluorescence lifetime $\left(\tau_{0}\right)$ of the excited states as a function of temperature. At each temperature, $\tau_{0}(T)$ was measured for 10 emitters. The error bars denote the standard deviation of the $\tau_{0}(T)$ distribution. The fit (magenta) line corresponds to the Mott-Seitz model. (b) Optical transition wavelength of transition $\mathrm{C}$ determined from Lorentzian fits to the spectrum and excitation scans. A cubic (magenta) dependence on temperature $\left(\sim T^{3}\right)$ is in good agreement with the data, unlike quadratic (dashed, orange) and quartic (dashed, brown) fits. (c,d) The measured excited and ground state splittings as a function of temperature. The quadratic fits based on the model in Section 5.2.4 are shown in magenta. The dashed green lines in (b), (c) and (d) are the best fits obtained using the pure thermal expansion mechanism explained in the text. 


\subsubsection{Optical line positions}

The line positions of all four optical transitions were determined using the Lorentzian fits to the measurements described in Section 5.2.1. The spectrometer was calibrated with respect to a wavemeter, allowing us to consistently reproduce transition wavelengths across the entire temperature range. For simplicity, only transition $\mathrm{C}$ is shown in Fig. 5.4(b). Fitting with a free temperature exponent results in $\Delta \lambda \sim(T / K)^{2.78 \pm 0.05}$, in close agreement with a cubic temperature dependence of the line position. Fig. 5.4(b) compares fits of the form $\Delta \lambda \sim T^{\alpha}$ for $\alpha=2,3,4$ as well as a model based on thermal expansion described below. Our observation of $T^{3}$ scaling differs marginally from earlier measurements made on nanodiamonds [153]. For temperatures at which the linewidth was narrow enough to resolve individual transitions, the ground and excited state splittings could also be obtained from the spectrum. We observe that the measured splittings, which correspond to the spin-orbit interaction at low temperature, are reduced with increasing temperature for both the excited (Fig. 5.4(c)) and ground (Fig. 5.4(c)) states.

In diamond, the temperature shifts of optical lines have two distinct origins: thermal expansion and electron-phonon interactions $[115,158]$. The shift of the transition energy due to thermal expansion has the form $\delta E_{\text {exp. }}(T)=A \cdot P(T)$, where $A$ is the hydrostatic pressure shift of the transition energy, $P(T)=-B \int_{0}^{T} e(x) d x$ is the negative pressure of thermal expansion, $B$ is the diamond bulk modulus and $e(T)$ is the bulk thermal expansion coefficient $[115,158]$. Whilst $B$ and $e(T)$ are well-known for diamond [159], the pressure shift $A$ of the $\mathrm{SiV}$ optical transition has not been measured. The dashed green line in Fig. 5.4(b) is the best fit of the line shift ob- 
tained using the single fit parameter $A$ of the thermal expansion mechanism, and it is clear this does not account for the observed shift. The shift of the transition energy due to electron-phonon interactions typically arises from quadratic interactions with $\mathrm{A}_{1}$-symmetric phonon modes and produces a $T^{4}$ dependence [160], which is also inconsistent with our observations. Furthermore, a linear combination of shifts caused by these two mechanisms is not able to produce a good fit to our observations. It is shown in Section 5.3 that the atypical $T^{3}$ shift arises from second-order linear interactions with E-symmetric phonon modes. This fits well to the observed data and therefore the shift due to thermal expansion is negligible.

The temperature reductions of the ground and excited state splittings can also arise from thermal expansion and electron-phonon interactions [158]. The dashed green lines in Fig. 5.4(c) and (d) are the best thermal expansion fits obtained by introducing pressure shift parameters of the spin-orbit splittings, and as above it is clear, at least for Fig. 5.4(c), that another mechanism must be involved in the reduction of the splittings. Similarly, the $T^{4}$ dependence of the quadratic interactions with $\mathrm{A}_{1}$-symmetric phonon modes and its linear combination with the thermal expansion shift do not satisfactorily fit the observations. We will show in the next section that the $T^{2}$ dependence of the spin-orbit splittings are also consequences of second-order linear interactions with E-symmetric phonon modes as for the shift of the transition energy. 


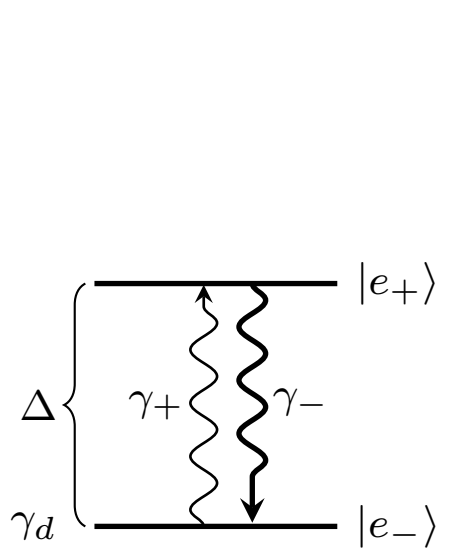

(a)

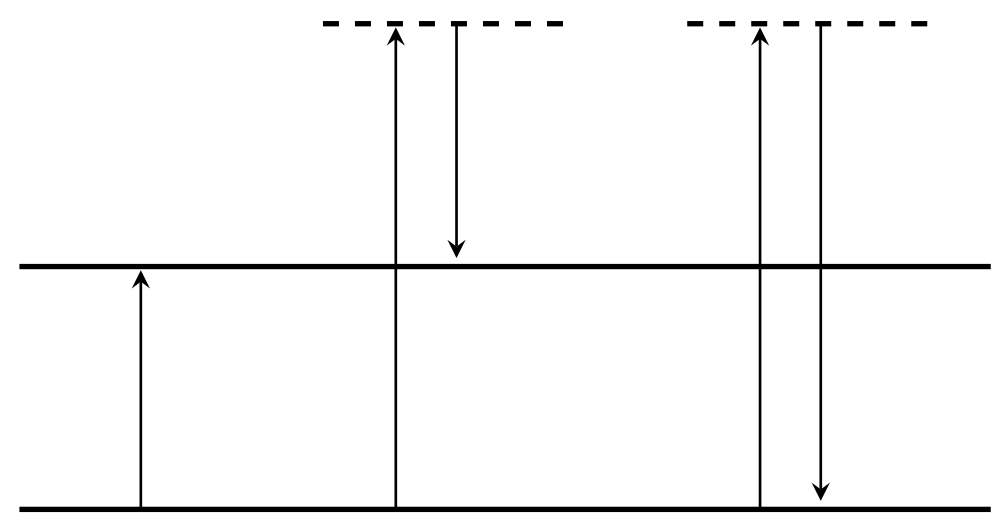

(b)

(c)

(d)

Figure 5.5: Electron-phonon processes within the ground and excited states. (a) The relevant rates in the problem are $\gamma_{+}$and $\gamma_{-}$, which denote the transition rates between states that determine the orbital $T_{1}$ relaxation time, and $\gamma_{d}$ which denotes the rate of a pure dephasing process. These rates can originate from a (b) single-phonon direct process; (c) two-phonon Raman process; or (d) two-phonon elastic scattering process. These processes are analogous to (b) resonant absorption, (c) Raman transitions, and (d) AC Stark shift in atomic physics.

\subsection{Microscopic model of the electron-phonon pro-}

\section{cesses}

In this section we develop a microscopic model of the electron-phonon processes within the ground and excited electronic levels that are summarised in Fig. 5.5. The model successfully describes the observed temperature variations of optical linewidth, line position and spin-orbit splittings. In each case, the electron-phonon processes are consequences of the linear Jahn-Teller interaction between the E-symmetric electronic states and E-symmetric acoustic phonon modes [150, 89, 108].

As discussed in the introduction, spin projection and orbital angular momentum are good quantum numbers and can be treated separately in the situation of low 
magnetic fields and strain [58]. Since the optical transitions and electron-phonon interactions are spin conserving, we can focus on the orbital degrees of freedom of the ground and excited levels. For a given spin state, the effective zero-field orbital Hamiltonian takes the following form for both the ground and excited levels

$$
H_{0}= \pm \frac{1}{2} \hbar \Delta \sigma_{z}
$$

where $\sigma_{z}$ is the usual Pauli operator for orbital states in the $\left\{\left|e_{+}\right\rangle,\left|e_{-}\right\rangle\right\}$basis, $\hbar \Delta$ is the magnitude of the relevant spin-orbit splitting, which is $+\hbar \Delta$ for $|\uparrow\rangle$ and $-\hbar \Delta$ for $|\downarrow\rangle$.

\subsubsection{Electron-phonon interaction}

For the $\mathrm{SiV}$ centre the interaction between the E-symmetric orbital states $\left\{\left|e_{+}\right\rangle,\left|e_{-}\right\rangle\right\}$ and phonon modes of E symmetry is described most easily if the E modes are linearly transformed to be circularly polarised. With this transformation, the phonon Hamiltonian and the linear electron-phonon interaction are

$$
\begin{gathered}
\hat{\mathrm{H}}_{\mathrm{E}}=\sum_{p, k} \hbar \omega_{k} a_{p, k}^{\dagger} a_{p, k} \\
\hat{\mathrm{V}}_{\mathrm{E}}=\sum_{k} \hbar \chi_{k}\left[\sigma_{+}\left(a_{-, k}+a_{-, k}^{\dagger}\right)+\sigma_{-}\left(a_{+, k}+a_{+, k}^{\dagger}\right)\right],
\end{gathered}
$$

where $\chi_{k}$ is the interaction frequency for a single phonon, $\sigma_{+}\left(\sigma_{-}\right)$is the raising (low-

ering) operator for orbital states, and $a_{p, k}^{\dagger}\left(a_{p, k}\right)$ is the creation (annihilation) operator for phonons with polarisation $p=\{-,+\}$ and wavevector $k$. Long wavelength acoustic phonons in diamond give rise to the collective translation and relative displacement of the ions of the center. Phonon modes resulting in the relative displacement have 
even parity and are responsible for the electron-phonon coupling. The interaction frequency and density of modes are approximately $\overline{\left|\chi_{k}(\omega)\right|^{2}} \approx \chi \omega$ and $\rho(\omega)=\rho \omega^{2}$, respectively, where the overbar denotes the average over all modes with frequency $\omega_{k}=\omega$ and $\chi$ and $\rho$ are proportionality constants $[85,161]$. Note that interactions with A-symmetric modes have not been included as they do not couple the states within the ground and excited electronic levels.

\subsubsection{First-order electron-phonon transitions}

Treating $\hat{V}_{\mathrm{E}}$ as a time-dependent perturbation, the first-order transitions between the orbital states involve the absorption or emission of a single $\mathrm{E}$ phonon whose frequency is resonant with the splitting $\Delta$ (see Fig. 5.5 (b)). The corresponding transition rates are

$$
\begin{gathered}
\gamma_{+}=2 \pi \sum_{k} n_{-, k}\left|\chi_{k}\right|^{2} \delta\left(\Delta-\omega_{k}\right) \\
\gamma_{-}=2 \pi \sum_{k}\left(n_{+, k}+1\right)\left|\chi_{k}\right|^{2} \delta\left(\Delta-\omega_{k}\right),
\end{gathered}
$$

where $n_{p, k}$ is the occupation of the phonon mode with polarisation $p$ and wavector $k$. Assuming acoustic phonons, performing the thermal average over initial states and the sum over all final states leads to

$$
\begin{gathered}
\gamma_{+}=2 \pi \chi \rho \Delta^{3} n(\Delta, T) \\
\gamma_{-}=2 \pi \chi \rho \Delta^{3}[n(\Delta, T)+1] .
\end{gathered}
$$

For temperatures $T>\hbar \Delta / k_{\mathrm{B}}$, Eq. (5.5) can be approximated by a single relaxation rate with a linear temperature dependence

$$
\gamma_{+} \approx \gamma_{-} \approx \frac{2 \pi}{\hbar} \chi \rho \Delta^{2} k_{\mathrm{B}} T .
$$


Hence, the one-phonon transitions lead to the relaxation of population between the orbital states as well as the dephasing of the states that are linearly dependent on temperature. The measurements presented in Fig. 5.2 and Fig. 5.3 demonstrated a clear linear dependence of broadening for temperatures below $20 \mathrm{~K}$, but greater than the spin orbit splitting $\left(T>\hbar \Delta / k_{\mathrm{B}} \sim 2.4 \mathrm{~K}\right)$. We therefore conclude that the relaxation mechanisms are dominated by a resonant single phonon process at liquid helium temperatures for both the ground and the excited states. Eq. (5.6) also shows that the relaxation rate is $\sim \Delta^{2}$, where $\Delta$ is the spin-orbit splitting in the zero-field limit. The $\Delta^{2}$ scaling explains why the phonon relaxation processes are much faster in the excited levels for which the splittings are larger compared with the ground states.

\subsubsection{Second-order electron-phonon transitions}

It was seen in Fig. 5.2 that the line broadening deviated from its linear temperature dependence above about $20 \mathrm{~K}\left(T \gg \hbar \Delta / k_{\mathrm{B}}\right)$, suggesting that higher order processes involving two phonons start dominating the relaxation rates. Given the form of the electron-phonon interaction in Eq. (5.3), the only allowed two-phonon processes are those where the initial and final orbital states are identical. Therefore, the inelastic Raman-type scattering processes [Fig. 5.5(c)] that are dominant

for NVcentres [85], are suppressed in $\mathrm{SiV}$ and the elastic Raman-type scattering pro- 
cesses [Fig. 5.5(d)] dominate instead. The elastic scattering rate for $\left|e_{-}\right\rangle$is

$$
\begin{aligned}
& \gamma_{d-}=2 \pi \hbar^{2} \sum_{k, q} \quad n_{-, k}\left(n_{+, q}+1\right)\left|\chi_{k}\right|^{2}\left|\chi_{q}\right|^{2} \\
& \left|\frac{1}{\Delta-\omega_{k}}+\frac{1}{\Delta+\omega_{k}}\right|^{2} \delta\left(\Delta-\omega_{k}+\omega_{q}\right) .
\end{aligned}
$$

Performing the thermal average over the initial states and the sum over all final states leads to

$$
\begin{gathered}
\gamma_{d-}=2 \pi \hbar^{2} \int_{0}^{\Omega} n(\Delta+\omega, T)(n(\omega, T)+1) \overline{\left|\chi_{k}(\Delta+\omega)\right|^{2}} \overline{\left|\chi_{q}(\omega)\right|^{2}} \\
\left|\frac{1}{-\omega}+\frac{1}{\Delta+\omega}\right|^{2} \rho(\Delta+\omega) \rho(\omega) \mathrm{d} \omega
\end{gathered}
$$

where $\Omega$ is the Debye frequency of diamond. Assuming acoustic modes and that the temperatures are such that only modes with frequencies $\Omega \gg \omega \gg \Delta$ contribute significantly to the integral, to lowest order in $\Delta$, the rates become

$$
\begin{gathered}
\gamma_{d-} \approx \gamma_{d+} \approx 2 \pi \hbar^{2} \Delta^{2} \chi^{2} \rho^{2} \int_{0}^{\infty} n(\omega, T)(n(\omega, T)+1) \omega^{2} \mathrm{~d} \omega \\
=\frac{2 \pi^{3}}{3 \hbar} \Delta^{2} \chi^{2} \rho^{2} k_{\mathrm{B}}^{3} T^{3} .
\end{gathered}
$$

Hence, the two-phonon elastic scattering process contribute to the dephasing of the orbital states and have rates that are proportional to $\sim T^{3}$, matching the observed linewidth behaviour in Fig. 5.2. Therefore our microscopic model shows perfect agreement with the measurements and we can understand the orbital relaxation process as a combination of a single phonon mixing between the orbital states and a two-phonon dephasing process.

For SiV centres under high strain (larger than the spin-orbit interaction), the orbital eigenstates $\left\{\left|e_{x}\right\rangle,\left|e_{y}\right\rangle\right\}$ no longer have well defined angular momentum. Under such conditions, the inelastic Raman process shown in Fig. 5.5(c) becomes allowed, which results in a competing orbital relaxation rate that scales as $\sim T^{5}$. 


\subsubsection{Spin-orbit splitting shifts}

The electron-phonon interactions also perturb the energies of the orbital states at second-order. The second-order energy shifts $\delta E_{-}\left(\delta E_{+}\right)$for states $\left|e_{-}\right\rangle\left(\left|e_{+}\right\rangle\right)$can be expressed in a simple form using the linear phonon $\{x, y\}$ polarisation basis. The energy shift due to phonon modes with wavevector $k$ and occupation $n_{x(y), k}$ are

$$
\begin{gathered}
\delta E_{-}(x(y), k)=\hbar^{2} \chi_{k}^{2}\left(\frac{n_{x(y), k}}{\omega-\Delta}-\frac{n_{x(y), k}+1}{\omega+\Delta}\right) \\
\delta E_{+}(x(y), k)=\hbar^{2} \chi_{k}^{2}\left(\frac{n_{x(y), k}}{\omega+\Delta}-\frac{n_{x(y), k}+1}{\omega-\Delta}\right),
\end{gathered}
$$

where each polarisation contributes independently. Assuming acoustic modes and that the temperatures are such that only modes with frequencies $\Omega \gg \omega \gg \Delta$ contribute significantly to the integral, then correct to lowest order in $\Delta$, the thermal averages of the shifts in the orbital energies over all (acoustic) vibrational levels are

$$
\begin{gathered}
\overline{\delta E_{-}}=\hbar^{2} \chi \rho\left(-\frac{1}{3} \Omega^{3}+\frac{\Delta}{2} \Omega^{2}+\frac{\pi^{2} k_{B}^{2}}{3 \hbar^{2}} T^{2}\right) \\
\overline{\delta E_{+}}=-\hbar^{2} \chi \rho\left(\frac{1}{3} \Omega^{3}+\frac{\Delta}{2} \Omega^{2}+\frac{\pi^{2} k_{B}^{2}}{3 \hbar^{2}} T^{2}\right) .
\end{gathered}
$$

This yields a temperature shift in the spin-orbit splitting

$$
\delta \Delta=\overline{\delta E_{+}}-\overline{\delta E_{-}}=-\hbar^{2} \chi \rho \Delta\left(\Omega^{2}+\frac{2 \pi^{2} k_{B}^{2}}{3 \hbar^{2}} T^{2}\right)
$$

that is proportional to $T^{2}$ and a temperature independent mean energy of the orbital states $\left(\overline{\delta E_{+}}+\overline{\delta E_{-}}\right) / 2=-\hbar^{2} \chi \rho \Omega^{3} / 3$. This correctly predicts the observed $T^{2}$ dependence of the fine structure splittings in Fig. $5.4(\mathrm{c}, \mathrm{d})$, but it fails to predict the $T^{3}$ dependence of the optical line position in Fig. 5.4(b). 


\subsubsection{Optical line position}

The failure of the above analysis to predict the temperature shift of the optical line position is due to a well known problem in the treatment of the linear JahnTeller interaction [162]. The problem arises from the implicit choice of rectangular mode coordinates for the zero-order vibrational wavefunctions of the perturbative analysis. In rectangular coordinates, the vibrational wavefunction of a pair $\left(Q_{x}, Q_{y}\right)$ of degenerate $\mathrm{E}$ modes is of the form $\psi_{i}\left(Q_{x}\right) \psi_{j}\left(Q_{y}\right)$, where $i$ and $j$ are the independent vibrational quantum numbers of the modes. Since the rectangular coordinates do not match the cylindrical symmetry of the linear Jahn-Teller vibrational potential, the rectangular vibrational wavefunctions are a poor choice of zero-order basis [163]. As a consequence, much higher perturbative expansions are required to correctly predict a shift in the optical line position.

A superior choice of basis is obtained by transforming to polar coordinates $\left(Q_{x}, Q_{y}\right) \rightarrow(\rho, \phi)$, within which the vibrational wavefunctions take the form $\psi_{\nu, l}(\rho, \phi)$, where $\nu=1,2, \ldots$ is the principal vibrational quantum number and $l=-\nu+1,-\nu+2, \ldots, \nu-1$ is the vibrational angular momentum quantum number, such that the vibrational energies of modes with frequency $\omega$ are $E_{\nu}=\nu \hbar \omega$ [163]. Using the formalism of the linear Jahn-Teller effect in the polar vibrational basis [163], we obtained the second-order shifts of the vibrational energies as per Section 5.3.4. Performing the thermal average of the shifts in the orbital energies over all (acoustic) vibrational levels, the corrected expression for the temperature shift of the optical line position is

$$
\frac{1}{2}\left(\overline{\delta E_{+}}+\overline{\delta E_{-}}\right)=-2 \hbar \chi \rho \int_{0}^{\Omega} \frac{2 e^{\hbar \omega / k_{B} T}\left(e^{2 \hbar \omega / k_{B} T}+3\right)}{\left(e^{\hbar \omega / k_{B} T}-1\right)\left(e^{\hbar \omega / k_{B} T}+1\right)^{2}} \omega^{2} d \omega \propto T^{3},
$$

which correctly predicts the $T^{3}$ dependence of the optical line position. Note that 
this corrected approach is consistent with the previous subsection and also predicts a $T^{2}$ dependence of the fine structure splittings. Hence, we can conclude that the electron-phonon processes of the linear Jahn-Teller interactions within the ground and excited electronic levels are responsible for the observed temperature variations of the optical linewidth, position and fine structure splittings.

\subsection{Discussion}

In Section 5.3, we have shown that a simple model of linear electron-phonon interactions can be used to successfully explain population dynamics $\left(\gamma_{+,-}^{e, g}\right.$, Section 5.3.2), dephasing processes $\left(\gamma_{d}^{e, g}\right.$, Section 5.3.3), relative (Section 5.3.4) and mean (Section 5.3.5) energy shifts within the ground and excited states. We next discuss implications of our observations for ground state coherences and approaches that could be used to enhance coherence times.

\subsubsection{Implications for ground state coherences}

The SiV ground states have spin and orbital degrees of freedom which can be used as qubit states. Fig. 5.6(a) shows the electronic states under a magnetic field applied along the $\mathrm{SiV}$ symmetry axis. The orbital relaxation rates $\gamma_{+,-}^{g, e}$ discussed in this manuscript are spin conserving, consistent with the long spin $T_{1}$ times that were recently measured [61]. Even though such orbital relaxations are spin conserving, the

detuning between $|\uparrow\rangle$ and $|\downarrow\rangle$ spin states varies depending on which orbital state is occupied. This means that the phase evolution of any coherent spin state changes after a phonon-induced orbital quantum jump, leading to the accumulation of random 
(a)

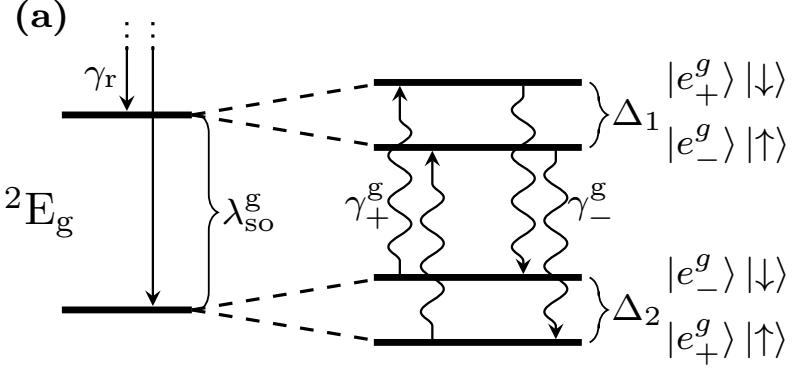

(b)

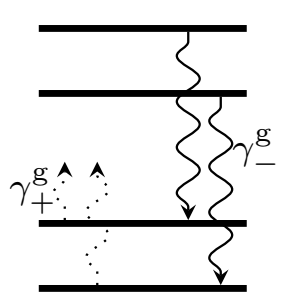

(c)

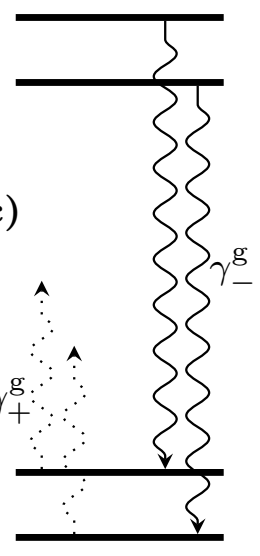

Figure 5.6: (a) Implications of phonon processes for ground state coherences. The transitions arising from electron-phonon interactions described by our model are spin conserving. Any coherences created between two ground states decay with the orbital relaxation rates. (b) The $\gamma_{+}^{g}$ can be suppressed at low temperatures $\left(T \ll \Delta=\lambda_{\mathrm{SO}}^{\mathrm{g}}\right)$. (c) Large strain fields result in an increased splitting, also resulting in reduced phonon occupation and suppressed $\gamma_{+}^{g}$. For (b) and (c), the two lowest energy states constitute a subspace with reduced thermal relaxation and extended coherence.

phase which is measured as decoherence. Hence coherences formed between any of the four states shown in Fig. 5.6 are expected to be limited by the $T_{1}$ of the orbital degree of freedom.

Recent experiments $[62,61]$ that probed ground state coherences using coherent population trapping $(\mathrm{CPT})$ have reported $T_{2}^{*}$ values that are in good agreement with the orbital $T_{1}$ reported in our work. We note that the model used in [62] considered thermal relaxation mechanisms only between the two lowest energy ground states shown in Fig. 5.6. The authors concluded thermal relaxation rates between these two states to be suppressed owing to small spin overlap at low magnetic fields, and the $T_{2}^{*}$ to be limited by magnetic field noise from the SiV environment. While the former agrees with our model (no relaxation between $\left|e_{-}\right\rangle|\downarrow\rangle \leftrightarrow\left|e_{+}\right\rangle|\uparrow\rangle$ ), all four 
ground states need to be considered to relate orbital relaxation rates to coherences. Based on the close experimental agreement between $T_{2}^{*}$ and orbital $T_{1}$, we conclude that coherence times of $\mathrm{SiV}$ ground states are limited by phonon processes even at liquid helium temperatures.

\subsubsection{Extending ground state coherences}

We have shown that ground state coherences are limited by a single-phonon orbital relaxation process with a rate determined by a combination of phonon density of states and occupation $\left(\gamma_{ \pm}^{g} \sim \rho(\Delta)(2 n(\Delta, T)+1 \mp 1)\right)$ at the energy of the spin-orbit splitting with $\Delta=\lambda_{\mathrm{so}}^{\mathrm{g}} \sim 50 \mathrm{GHz}$. Since the interaction with the phonon bath is a Markovian process, dynamical decoupling sequences cannot be applied to extend coherences. To extend $T_{2}^{*}$, we will therefore focus on approaches that reduce the orbital relaxation rates $\gamma_{ \pm}^{g}$.

The first two approaches focus on reducing phonon occupation to decrease $\gamma_{+}^{g}$. The occupation depends on the ratio, $T / \Delta$, of the temperature and the energy splitting between the coupled orbital states. Substantial improvements can be achieved by minimizing this ratio in cooling the sample to lower temperatures $(T \ll \Delta \sim 2.4 \mathrm{~K}$, Fig. 5.6(b)). Based on our fits in Section 5.2.2, the expected orbital relaxation timescale is given by $1 / \gamma_{+}^{g}=101\left(e^{2.4 / T}-1\right)$ ns which correspond to $1 \mu$ s at $1 \mathrm{~K}$ and $1 \mathrm{~ms}$ at $0.26 \mathrm{~K}$. A second approach is to increase $\Delta$ by using emitters subject to high strain. At the limit of $\Delta \gg T$, similar reductions in phonon occupation can be used to suppress relaxation rates as shown in Fig. 5.6(c). Based on Eq. (5.5), we find that $1 / \gamma_{+}^{g}$ equals $1 \mathrm{~ms}(1 \mu \mathrm{s})$ for a strain shift of $1.6 \mathrm{THz}(0.9 \mathrm{THz})$ at $4 \mathrm{~K}$. We note that 
in both cases, only the two lowest energy states constitute a subspace that does not couple to phonons. The lowest two energy states are therefore expected to have long coherence times and could be used as a long-lived spin qubit.

The linear interaction Hamiltonian of Section 5.3.1 and the resulting single-phonon orbital relaxation process are analogous to the Jaynes-Cummings Hamiltonian and Wigner-Weisskopf model of spontaneous emission used in quantum optics [164]. One can therefore use ideas developed in the context of cavity QED to engineer relaxation rates $\gamma_{ \pm}^{g}$. In particular, the phonon density of states can be reduced to suppress the orbital relaxation rates. This is analogous to inhibited spontaneous emission of photons [165] which has been observed for microwave and optical photons in atomic and solid-state systems $[166,167,168]$. Acoustic phonons in diamond offer an exciting new platform to probe this effect in a new regime owing to the highly broadband and reflective boundary conditions at the diamond-vacuum interface. To suppress orbital relaxation rates due to phonons at $\Delta \sim 50 \mathrm{GHz}$, small nano diamonds $(d<120 \mathrm{~nm})$ can be used to realise a complete phononic band gap for $\nu<50 \mathrm{GHz}$ phonons owing to the strong confinement [169]. An alternative approach would utilise recent advances in diamond nanofabrication $[117,170]$ to create 1D-optomechanical structures engineered to inhibit phonon and enhance optical transitions by modifying the density of states $[171,172,173,174]$. Using this approach both $\gamma_{+}^{g}$ and $\gamma_{-}^{g}$ are inhibited, therefore all four ground states can be used as long-lived qubits. We expect both approaches that modify phonon occupation and density of states to result in substantial improvements for the ground state coherences of $\mathrm{SiV}$ centres. 


\title{
Chapter 6
}

\section{Narrow-linewidth homogeneous}

\author{
optical emitters in diamond
}

\section{nanostructures via silicon ion}

\section{implantation}

\subsection{Introduction}

Coherent emitters of indistinguishable single photons are a basic ingredient in many quantum information systems[175]. Atom-like emitters in the solid state are a particularly appealing platform for practical quantum information because they can be scalably integrated into nanophotonic devices. However, no single solid-state system has yet combined high brightness of narrowband emission and a low inhomogeneous distribution of photon frequencies from separate emitters (indistinguishabil- 
Chapter 6: Narrow-linewidth homogeneous optical emitters in diamond nanostructures via silicon ion implantation

ity) with ease of incorporation into nanophotonic structures on demand. For example, optically active semiconductor quantum dots can be bright and integrable into nanostructures, but have a large inhomogeneous distribution[35]. Nitrogen-vacancy (NV) centers in bulk diamond[105] are bright and photostable, with a moderate inhomogeneous distribution that allows straightforward tuning of multiple NV centers into resonance. These properties allow proof-of-principle demonstrations of quantum information protocols such as remote spin-spin entanglement generation $[65,66]$ and quantum teleportation[123]. Further progress towards developing NV based quantum devices has been hindered by low indistinguishable photon generation rates associated with the weak NV zero-phonon line, a challenge that could be addressed by integrating NV centers into nanophotonic structures. However, the optical transition frequencies of NV centers are very sensitive to their local environment[48, 129], making integration of spectrally stable emitters into nanophotonic structures a major challenge[49].

The negatively charged silicon-vacancy color center in diamond (SiV) has shown promise in fulfilling the key criteria of high brightness[55], lifetime-limited optical linewidths[176], and a narrow inhomogeneous distribution of optical transition frequencies[128]. The SiV (Fig. 6.1) has electronic states with strong dipole transitions where $70 \%$ of the emission is in the zero-phonon line (ZPL) at $737 \mathrm{~nm}[55]$. The inversion symmetry of the SiV prevents first-order Stark shifts, suppressing spectral diffusion[176] and allowing indistinguishable photons to be generated from separate emitters without the need for tuning or extensive pre-selection of emitters[142]. When combined with a spin degree of freedom[126], the SiV center's bright narrowband tran- 
Chapter 6: Narrow-linewidth homogeneous optical emitters in diamond nanostructures via silicon ion implantation

sition, narrow inhomogeneous distribution, and spectral stability make it a promising candidate for applications in quantum optics and quantum information science.

Silicon-vacancy centers occur only rarely in natural diamond[177], and are typically introduced during CVD growth via deliberate doping with silane[137, 148] or via silicon contamination[176, 153, 152, 128, 178]. While these techniques typically result in a narrow inhomogeneous distribution of $\mathrm{SiV}$ fluorescence wavelengths, these samples have a number of disadvantages. For example, the concentration of $\mathrm{SiV}$ centers can be difficult to control and localization of $\mathrm{SiV}$ centers in three dimensions is impossible.

Ion implantation offers a promising solution to these problems. By controlling the energy, quantity, and isotopic purity of the source ions, the depth, concentration, and isotope of the resulting implanted ions can be controlled. Ion implantation is widely commercially available. Targeted ion implantation using a focused silicon ion beam is also possible, allowing for placement of silicon defects in all three dimensions with precision on the scale of tens of nanometers[179]. Despite the advantages of ion implantation, there have been conflicting results[180, 179, 58] on the brightness and creation yield of $\mathrm{SiV}$ centers produced using this method and no systematic studies of the inhomogeneous distribution of $\mathrm{SiV}$ fluorescence wavelengths. Although there has been a single report of an implanted $\mathrm{SiV}$ with a linewidth roughly 10 times the lifetime limit[62], to the best of our knowledge there has been up to now no consistent method for producing SiV centers with bright, narrow-linewidth emission using ion implantation. These two criteria of a low inhomogeneous distribution relative to the single-emitter linewidth and narrow single-emitter linewidth relative to the lifetime 
a.
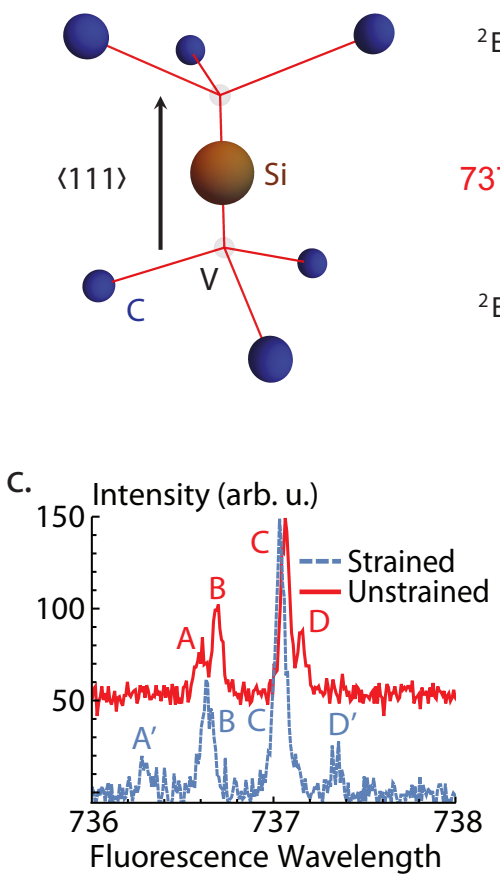
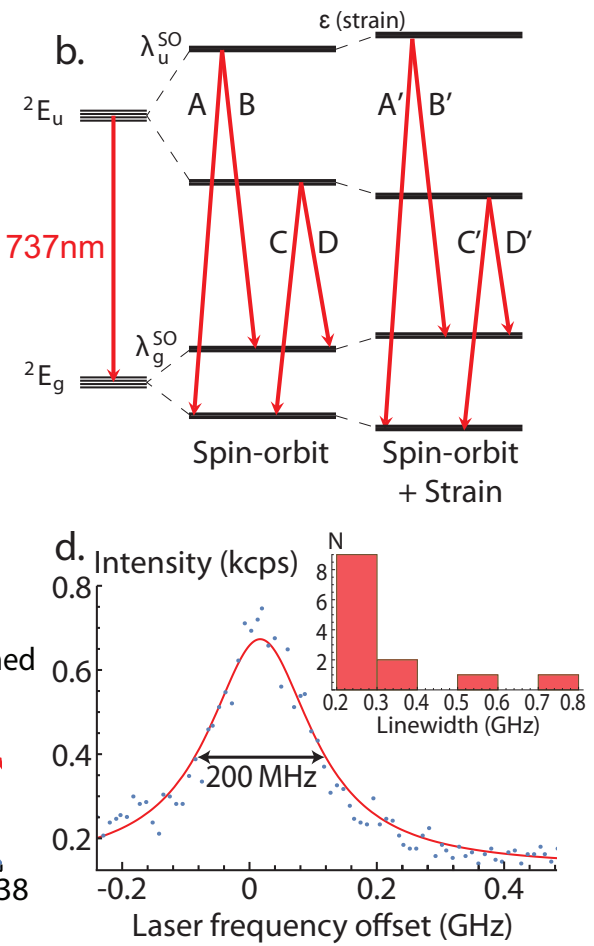

Figure 6.1: Properties of the SiV center. a. Atomic structure of the SiV center. The $\mathrm{V}-\mathrm{Si}-\mathrm{V}$ axis lies along the $\langle 111\rangle$ lattice direction. The SiV has $\mathrm{D}_{3 \mathrm{~d}}$ symmetry. b. Level structure of the $\mathrm{SiV}$ center. The $\mathrm{SiV}$ is a single-hole system with double orbital and spin degeneracy. This degeneracy is partially lifted by spin-orbit coupling $\left(\lambda_{g}^{S O}=\right.$ $47 \mathrm{GHz}$ and $\left.\lambda_{u}^{S O}=260 \mathrm{GHz}[58,176]\right)$. Lattice strain increases the splitting between these spin-orbit levels, shifting the transition frequencies. c. Fluorescence spectra of the ZPLs of single SiV centers in high-strain (blue, dashed) and low-strain (red) environments at $9-15 \mathrm{~K}$. Transitions B and $\mathrm{C}$ are less sensitive to strain compared with transitions $\mathrm{A}$ and $\mathrm{D}$ because the ground and excited states shift in the same (opposite) directions for transitions B and C (A and D)[128]. Unstrained spectrum offset and scaled vertically for clarity. d. Linewidth (FWHM) of representative implanted $\mathrm{SiV}$ in bulk (unstructured) diamond measured by PLE spectroscopy (blue points: data; red line: Lorentzian fit). Inset: histogram of emitter linewidths in bulk diamond. Almost all emitters have a linewidth within a factor of three of the lifetime limit $(94 \mathrm{MHz})$. 
Chapter 6: Narrow-linewidth homogeneous optical emitters in diamond nanostructures via silicon ion implantation

limit are essential for quantum optics applications $[175,91]$.

In this chapter, we report the creation of SiV centers in diamond using ion implantation. Implantation is followed by high-temperature high-vacuum annealing to facilitate SiV formation and repair implantation-induced damage to the lattice. The resulting emitters have narrow optical transitions within a factor of four of the lifetime limited linewidth and a narrow inhomogeneous distribution such that the half of the emitters have transitions that lie in a $15 \mathrm{GHz}$ window. Finally, we incorporate these $\mathrm{SiV}$ centers into nanostructures and demonstrate that their favorable optical properties are maintained even after fabrication.

The silicon-vacancy color center is a point defect in diamond wherein a silicon atom occupies an interstitial position between two vacancies (Fig. 6.1a)[124]. The $\mathrm{SiV}$ is a spin- $\frac{1}{2}$ system with ground $\left({ }^{2} \mathrm{E}_{g}\right)$ and excited $\left({ }^{2} \mathrm{E}_{u}\right)$ states localized to the diamond bandgap $[59,124,125]$. Both states have double spin and orbital degeneracies partially lifted by the spin-orbit interaction (Fig. 6.1b) which splits each quartet into two degenerate doublets. The spin-orbit splittings for the ground and excited states are 0.19 and $1.08 \mathrm{meV}(47$ and $260 \mathrm{GHz})$, respectively (Fig. 6.1c)[59, 58]. All transitions between the ground and excited states are dipole-allowed with a ZPL energy of $1.68 \mathrm{eV}(\lambda=737 \mathrm{~nm})$ and an excited state lifetime of under $1.7 \mathrm{~ns}[181]$. These optical transitions can have linewidths (Fig. 6.1d) comparable to the lifetime limit of $94 \mathrm{MHz}[176]$.

The $\mathrm{SiV}$ is sensitive to strain, which can both shift the average energy (for axial strain) and increase the splitting (for transverse strain) in the ground and excited state manifolds (Fig. 6.1b, last column) $[126,58]$. Transitions B and C within the 
Chapter 6: Narrow-linewidth homogeneous optical emitters in diamond nanostructures via silicon ion implantation

ZPL are relatively insensitive to transverse strain because their ground and excited states shift in the same direction: both upward for transition B and both downward for transition C (Fig. 6.1c)[128]. Transition C is between the lowest energy ground and excited states which are also isolated from the phonon bath at low temperatures[181]. This transition is therefore the most suitable for applications in quantum information science.

\subsection{Creating $\mathrm{SiV}$ centers with ion implantation}

We create $\mathrm{SiV}$ centers using the following procedure: First, we begin with a polished CVD diamond (Element Six Inc., $[N]_{S}^{0}<5 \mathrm{ppb},\{100\}$ oriented top face). Previous work suggests that mechanical polishing produces a strained and damaged layer close to the surface that results in reduced mechanical stability of nanofabricated structures[130]. We also expect that the strain introduced by mechanical polishing will lead to a larger inhomogeneous distribution of $\mathrm{SiV}$ wavelengths. We reduce this damage by removing $5 \mu \mathrm{m}$ of diamond through reactive ion etching, producing a smooth (under $1 \mathrm{~nm}$ RMS roughness) surface. More details on this technique can be found elsewhere[130, 54]. An otherwise identical control sample was also put through the same implantation procedure but without this pre-etching step. We then implant ${ }^{29} \mathrm{Si}^{+}$ions (Innovion Corporation) at a dose of $10^{10} \mathrm{ions} / \mathrm{cm}^{2}$ and an energy of $150 \mathrm{keV}$ resulting in an estimated depth of $100 \pm 20 \mathrm{~nm}[182]$.

After implantation, we clean the samples using an an oxidative acid clean (boiling $1: 1: 1$ perchloric: nitric: sulfuric acid)[183] and then perform two high-temperature high-vacuum $\left(\lesssim 10^{-6}\right.$ Torr $)$ anneals. The first anneal is at $800^{\circ} \mathrm{C}$ for eight hours 
Chapter 6: Narrow-linewidth homogeneous optical emitters in diamond nanostructures via silicon ion implantation

after a four-hour bake-out step at $400^{\circ} \mathrm{C}$. At $800^{\circ} \mathrm{C}$, vacancies are mobile[184, 185, 42] leading to the formation of $\mathrm{SiV}$ centers. The second anneal is the same as the first, but with an additional step at $1100{ }^{\circ} \mathrm{C}$ with a two-hour dwell time. At this temperature, divacancies and other defects can also anneal out[186, 187]. For all annealing steps, we use slow temperature ramps $\left(\lesssim 35^{\circ} \mathrm{C}\right.$ per hour $)$ to maintain low pressures in our furnace. This annealing procedure, inspired by previous work with $\operatorname{SiV}[188,152]$ and NV[186, 189, 190, 54] centers, both aids in the formation of SiV centers and also helps remove damage to the crystal lattice, reducing local strain. The residual graphitic carbon produced during these high-temperature anneals was removed by again performing the oxidative acid clean. Before each annealing step, we use X-ray photoelectron spectroscopy to verify that the surface is free of contaminants.

\subsection{Results and discussion}

\subsection{1 $\mathrm{SiV}$ centers in bulk diamond}

We confirm that the $\mathrm{SiV}$ centers exhibit narrow-linewidth optical transitions by performing photo-luminescence excitation (PLE) spectroscopy after $1100{ }^{\circ} \mathrm{C}$ annealing. In this experiment, we scan the frequency of a weak resonant laser (New Focus Velocity, linewidth $\Delta f \lesssim 25 \mathrm{MHz}$ over the course of the experiment, stabilized with a High Finesse WS7 wavemeter) across transition C and monitor the fluorescence on the phonon-sideband (PSB). We integrate over several scans to reconstruct the time-averaged shape and position of the SiV ZPL (Fig. 6.1d). We perform these measurements in a helium flow cryostat at a sample stage temperature of $3.7 \mathrm{~K}$ to 
Chapter 6: Narrow-linewidth homogeneous optical emitters in diamond nanostructures via silicon ion implantation

avoid phonon-induced broadening of the optical transition[181]. The emitters are resonantly excited below saturation to avoid power broadening. (See Appendix C for more experimental details.) We find that $\mathrm{SiV}$ centers in bulk diamond have narrow optical transitions with linewidths of $\Gamma / 2 \pi=320 \pm 180 \mathrm{MHz}$ (mean and standard deviation for $\mathrm{N}=13$ spatially resolved emitters). Almost all $\mathrm{SiV}$ centers have a linewidth within a factor of three of the lifetime limit (Fig. 6.1d, inset). As defined here, these linewidths include the effects of phonon broadening and all spectral diffusion that happens at any timescale during the course of the experiment (4-15 minutes).

We characterize the inhomogeneous distribution of the implanted SiV fluorescence wavelengths after each annealing step via photoluminescence spectroscopy. To perform these measurements, we excite the $\mathrm{SiV}$ centers using off-resonant light from a $700 \mathrm{~nm}$ diode laser. Off-resonant excitation at $520 \mathrm{~nm}$ is also possible. Using both of these wavelengths together results in a superlinear enhancement in the observed count rate, suggesting that the $520 \mathrm{~nm}$ laser may play a role in stabilizing the $\mathrm{SiV}$ charge state. The resulting fluorescence is sent to a spectrometer (Horiba iHR550, $0.025 \mathrm{~nm}$ resolution). We perform these measurements at 9-15 K.

After annealing at $800^{\circ} \mathrm{C}$, the observed distribution is broad, with about half of the emitter transition wavelengths lying within a $3-4 \mathrm{~nm}$ range (Fig. 6.2a, red dashed curve). Transition $\mathrm{C}$ was used where unambiguous identification was possible; otherwise, the brightest transition (which should correspond to transition C[128, 176]) was used. After the $1100{ }^{\circ} \mathrm{C}$ anneal, the distribution becomes more than 100 times narrower, with about half of the 13 measured emitters (transition $\mathrm{C}$ ) now lying in a $0.03 \mathrm{~nm}(15 \mathrm{GHz})$ window (Fig. $6.2 \mathrm{a}$ and $6.2 \mathrm{~b}$, blue solid curves). In both cases, we 
Chapter 6: Narrow-linewidth homogeneous optical emitters in diamond nanostructures via silicon ion implantation
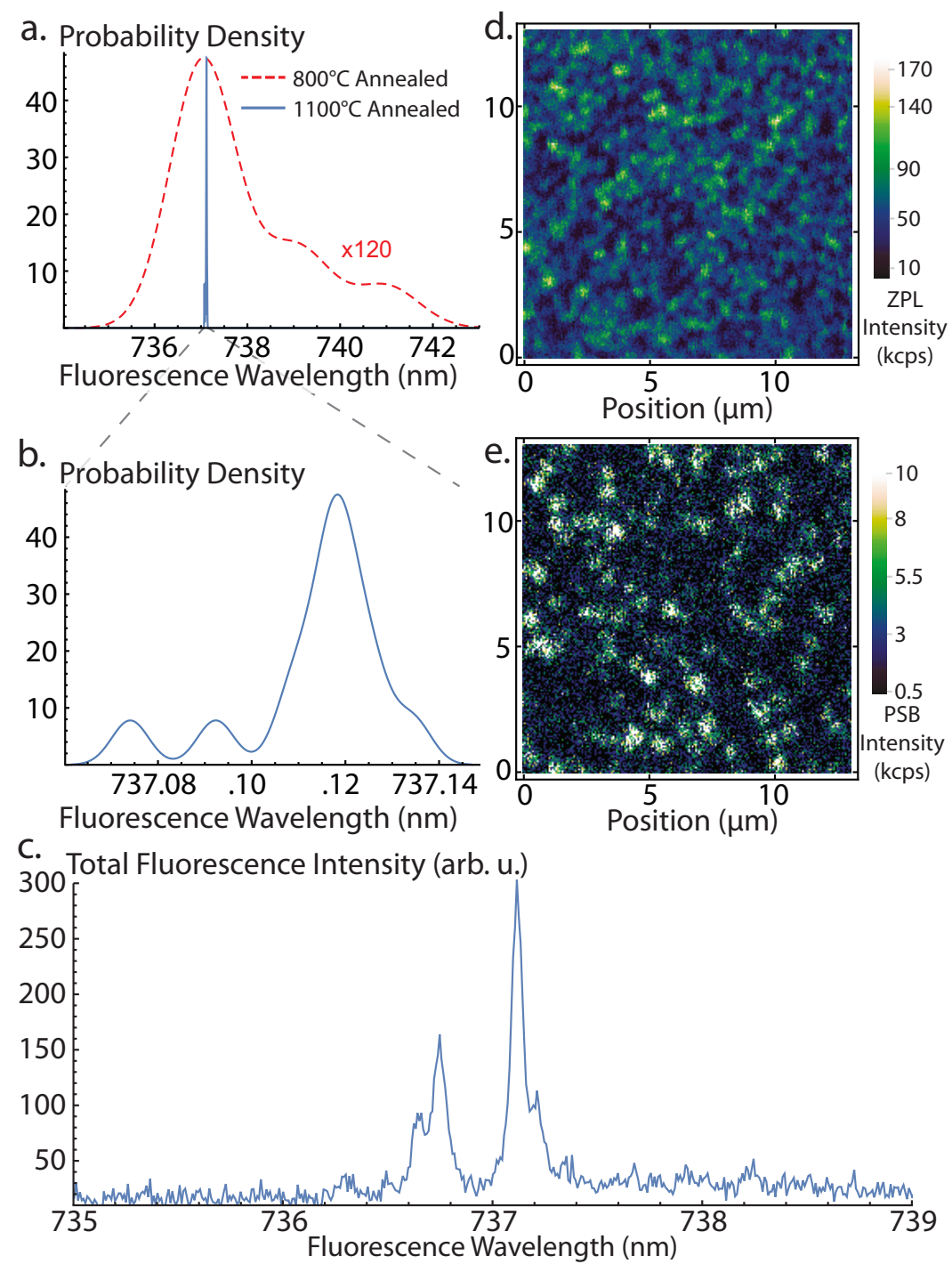

Figure 6.2: Inhomogeneous distribution of fluorescence wavelengths of implanted SiV transitions. a. Kernel density estimation of distribution of bulk SiV wavelengths after $800^{\circ} \mathrm{C}(\mathrm{N}=19$, red dashed curve $)$ and $1100^{\circ} \mathrm{C}(\mathrm{N}=13$, blue solid curve $)$ annealing. The distribution narrows from $3-4 \mathrm{~nm}\left(800^{\circ} \mathrm{C}\right.$ anneal $)$ to $0.03 \mathrm{~nm}\left(15 \mathrm{GHz}, 1100{ }^{\circ} \mathrm{C}\right.$ anneal). b. Zoomed-in distribution (transition C) after $1100^{\circ} \mathrm{C}$ annealing. Note the smaller wavelength range on the horizontal axis. c. Sum of spectra for different SiV centers after $1100{ }^{\circ} \mathrm{C}$ annealing. The $\mathrm{SiV}$ fine structure is clearly present, demonstrating that the inhomogeneous distribution is small. d, e. Spatial map of collected fluorescence (thousands of counts per second) over a region of bulk diamond exciting off (d) and on (e) resonance. By comparing the densities of emitters, we estimate that $30 \pm 15 \%$ of the emitters are nearly resonant. These measurements were taken at $9-15 \mathrm{~K}$. 
Chapter 6: Narrow-linewidth homogeneous optical emitters in diamond nanostructures via silicon ion implantation

focus on transition $\mathrm{C}$ because it is the brightest transition and relatively insensitive to strain[128] and phononic decoherence[181]. The other transitions are also much more narrowly distributed after $1100^{\circ} \mathrm{C}$ annealing. In Fig. 6.2c, we plot a composite spectrum constructed by summing over all of the normalized $13 \mathrm{SiV}$ spectra taken after $1100^{\circ} \mathrm{C}$ annealing. This composite spectrum is very similar to the spectrum of a single unstrained $\mathrm{SiV}$ center (Fig. 6.1c) and shows the expected fine-structure splitting, demonstrating that the inhomogeneous distribution of SiV transition wavelengths is small compared to the fine-structure splitting. This result is comparable to reported inhomogeneous distributions reported for $\mathrm{SiV}$ centers created during CVD growth $[176,142,128,58]$. It is possible that even higher temperature annealing could further reduce this inhomogeneous distribution[152, 190].

To estimate the yield of conversion from implanted $\mathrm{Si}^{+}$ions to $\mathrm{SiV}$ centers, we perform scanning confocal microscopy (Fig. 6.2d). Exciting with several milliwatts of off-resonant light $(700 \mathrm{~nm})$ gives around $10^{5}$ counts per second (cps) into a single-mode fiber from a single $\mathrm{SiV}$ in a $20 \mathrm{~nm}$ spectral range around the ZPL. In the resulting microscope image, we count the number of $\mathrm{SiV}$ centers and estimate a density of $0.5-1 / \mu \mathrm{m}^{2}$. Based on our $\mathrm{Si}^{+}$implantation density of $100 / \mu \mathrm{m}^{2}$, we estimate our $\mathrm{SiV}$ creation yield after $800^{\circ} \mathrm{C}$ annealing to be $0.5-1 \%$. There was no clear difference in the yield after performing the $1100{ }^{\circ} \mathrm{C}$ anneal. Furthermore, the yield in the sample that was not pre-etched was significantly higher $(2-3 \%)$. The observations that highertemperature annealing did not increase the yield and that the sample with greater surface damage had a larger yield both support the model that $\mathrm{SiV}$ formation is limited by the presence and diffusion of nearby vacancies[188, 187]. This yield could 
Chapter 6: Narrow-linewidth homogeneous optical emitters in diamond nanostructures via silicon ion implantation

be increased by electron irradiating the sample to create a higher vacancy density in a controllable way[186, 188, 148].

To visualize the density of nearly resonant $\mathrm{SiV}$ centers, we resonantly excited the $\mathrm{SiV}$ centers with a Rabi frequency of several $\mathrm{GHz}$ using an external-cavity diode laser tuned to the center of the inhomogeneous distribution. We scan spatially over the sample and collect fluorescence on the phonon side-band (PSB). The resulting image taken in the same region of the sample (Fig. 6.2e) has about a factor of three fewer emitters compared to the image taken with off-resonant excitation (N 100 vs. 340); roughly $30 \%$ of the emitters are near-resonant (within our few GHz Rabi frequency).

\subsection{2 $\mathrm{SiV}$ centers in nanostructures}

One major advantage of building quantum devices with solid-state emitters rather than trapped atoms or ions is that solid state systems are typically more easily integrated into nanofabricated electrical and optical structures[140, 191]. The scalability of these systems is important for practical realization of even simple quantum optical devices[192]. Unfortunately, many solid-state systems suffer serious deterioration in their properties when incorporated into nanostructures. For example, the large permanent electric dipole of $\mathrm{NV}$ centers in diamond causes coupling of the NV to nearby electric field noise, shifting its optical transition frequency as a function of time. The $\mathrm{SiV}$ is immune to this spectral diffusion to first order because of its inversion symmetry[142] and is therefore an ideal candidate for integration into diamond nanophotonic structures. Motivated by these considerations, we fabricated an array of diamond nanophotonic waveguides (Fig. 6.3a) on the pre-etched sample characterized 
Chapter 6: Narrow-linewidth homogeneous optical emitters in diamond nanostructures via silicon ion implantation

above using previously reported methods[130, 52]. Each waveguide (Fig. 6.3a, inset) is $23 \mu \mathrm{m}$ long with approximately equilateral-triangle cross sections of side length $300-500 \mathrm{~nm}$. After fabrication, we again performed the same $1100^{\circ} \mathrm{C}$ annealing and acid cleaning procedure. Many $\mathrm{SiV}$ centers are visible in a fluorescence image of the final structures (Fig. 6.3b). Photon correlation measurements (Appendix C) verify our ability to create and image single SiV centers.

To characterize the optical coherence properties of $\mathrm{SiV}$ centers in nanostructures, we again perform PLE spectroscopy. SiV centers in nanostructures have narrow transitions with a full-width at half-maximum (FWHM) of $\Gamma_{n} / 2 \pi=410 \pm 160 \mathrm{MHz}$ (mean and standard deviation for $\mathrm{N}=10$ emitters; see Fig. 6.3c inset for linewidth histogram), only a factor of 4.4 greater than the lifetime limited linewidth $\gamma / 2 \pi=$ $94 \mathrm{MHz}$. The linewidths measured in nanostructures are comparable to those measured in bulk (unstructured) diamond $\left(\Gamma_{b} / 2 \pi=320 \pm 180 \mathrm{MHz}\right)$. The ratios $\Gamma_{n} / \gamma$ and $\Gamma_{b} / \gamma$ are much lower than the values for NV centers, where the current state of the art for typical implanted NV centers in nanostructures[49] and in bulk[54] is $\Gamma_{n} / \gamma \gtrsim 100-200$ and $\Gamma_{b} / \gamma \gtrsim 10(\gamma / 2 \pi=13 \mathrm{MHz}$ for NV centers $)$.

It is possible for the lifetime in nanostructures to be longer than the lifetime in the bulk since the local photonic density of states is generally reduced inside such a structure[81, 193]. This potential change in lifetime would change the lifetime-limited linewidth and can also provide indirect evidence of the SiV quantum efficiency. To probe this effect, we measured the lifetime of nine $\mathrm{SiV}$ centers. The lifetime measured in the nanobeam waveguides $(\tau=1.69 \pm 0.14 \mathrm{~ns}, \mathrm{~N}=5)$ was not significantly different from the lifetime measured in the bulk-like anchors $(\tau=1.75 \pm 0.08 \mathrm{~ns}, \mathrm{~N}=4)$. Both 
Chapter 6: Narrow-linewidth homogeneous optical emitters in diamond nanostructures via silicon ion implantation
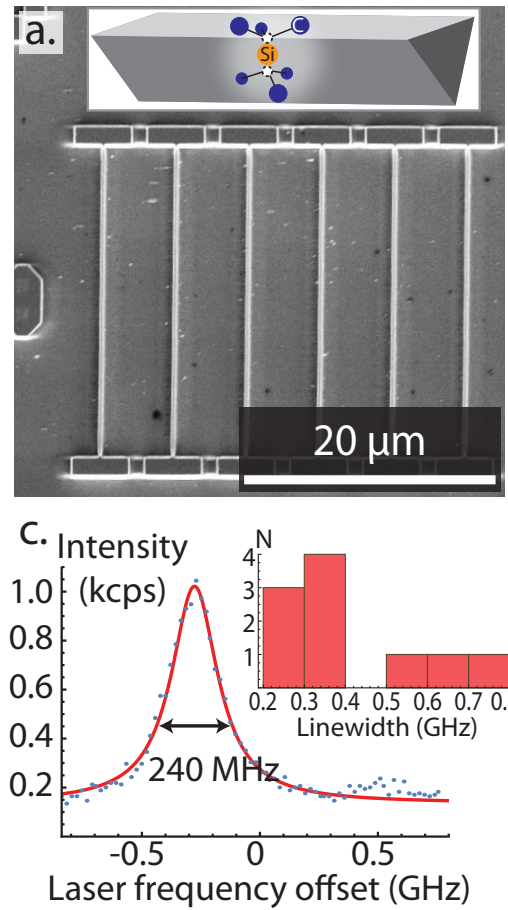

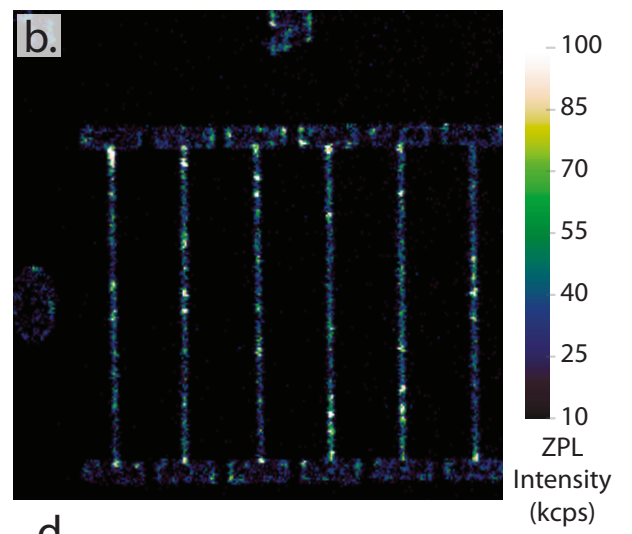

d. Laser frequency offset

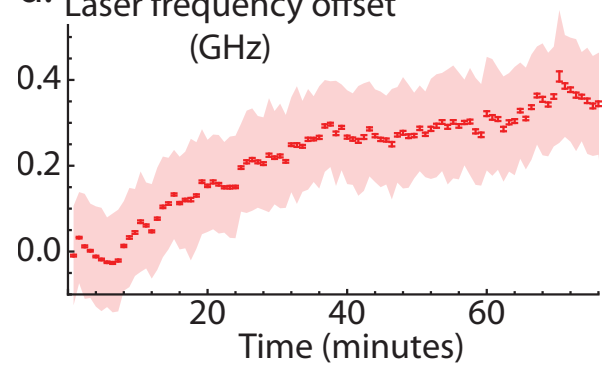

Figure 6.3: $\mathrm{SiV}$ centers in nanostructures. a. Scanning electron micrograph of six nanobeam waveguides. Inset: schematic of a triangular diamond nanobeam containing an SiV center. b. Spatial map of ZPL fluorescence collected by scanning confocal microscopy with off-resonant excitation. Multiple $\mathrm{SiV}$ centers are visible in each waveguide. c. Linewidth of representative implanted $\mathrm{SiV}$ inside a nano-waveguide measured by PLE spectroscopy (blue points: data; red line: Lorentzian fit). Inset: histogram of emitter linewidths in nanostructures. Most emitters have linewidths within a factor of four of the lifetime limit. d. Spectral diffusion of the emitter measured in part c. The total spectral diffusion is under $400 \mathrm{MHz}$ even after more than an hour of continuous measurement. This diffusion is quantified by measuring the drift of the fitted center frequency of resonance fluorescence scans as a function of time. Error bars are statistical error on the fitted center position. The lighter outline is the FWHM of the fitted Lorentzian at each time point. 
Chapter 6: Narrow-linewidth homogeneous optical emitters in diamond nanostructures via silicon ion implantation

values are in good agreement with the literature[181, 62].

By extracting the center frequency of each individual scan, we also determine the rate of fluctuation of the ZPL frequency and therefore quantify spectral diffusion (Fig. 6.3d). Optical transition frequencies in $\mathrm{SiV}$ centers are stable throughout the course of our experiment, with spectral diffusion on the order of the lifetime-limited linewidth even after more than an hour. Characterizing the inhomogeneous distribution of $\mathrm{SiV}$ centers in nanostructures is challenging because off-resonant excitation leads to strong background fluorescence, making exhaustive identification of all $\mathrm{SiV}$ centers in a given region difficult. Nevertheless, it is easy to find multiple SiV centers in nanostructures at nearly the same resonance frequency: to find the above ten emitters, we scanned the laser frequency over only a $20 \mathrm{GHz}$ range.

The residual broadening of the optical transition can result from a combination of second-order Stark shifts and phonon-induced broadening. The presence of a strong static electric field would result in an induced dipole that linearly couples to charge fluctuations, accounting for the slow diffusion. Finally, we expect that up to $50 \mathrm{MHz}$ of additional broadening could arise from the hyperfine interaction[61] present due to our choice of ${ }^{29} \mathrm{Si}$ ions. Determining the precise mechanisms limiting SiV linewidths is an important topic of future study.

To conclude, we have presented optical emission from implanted SiV centers with a narrow inhomogeneous distribution of $\mathrm{SiV}$ optical transition wavelengths and nearly lifetime-limited optical linewidths. These properties persist after nanofabrication, making the SiV center uniquely suited for integration into quantum nanophotonic devices[91, 194]. Recent advances in diamond fabrication technology[130, 131, 195] 
Chapter 6: Narrow-linewidth homogeneous optical emitters in diamond nanostructures via silicon ion implantation

suggest the tantalizing possibility of scalably integrating these high-quality implanted SiV centers into nanowire single photon sources[81] or nanocavities[196, 154]. Furthermore, combining our processing procedure with targeted implantation of silicon using a focused ion beam[179] either before or after fabrication[197] could significantly improve photonic device yields and reproducibility by deterministically positioning individual SiV centers in all three dimensions. Our work, combined with the promise of these future advances, could make the $\mathrm{SiV}$ center a new workhorse in solid-state quantum optics. 


\section{Chapter 7}

\section{An integrated diamond}

\section{nanophotonics platform for}

\section{quantum optical networks}

Efficient interfaces between photons and quantum emitters are central to applications in quantum science $[8,198]$ but are challenging to implement due to weak interactions between single photons and individual quantum emitters. Despite advances in the control of microwave and optical fields using cavity and waveguide quantum electrodynamics (QED) [134, 199, 37, 200, 201, 202, 203], the realization of integrated quantum devices where multiple qubits are coupled by optical photons remains an outstanding challenge[35]. In particular, due to their complex environments, solidstate emitters have optical transitions that generally exhibit a large inhomogeneous distribution[204, 35], rapid decoherence[201] and significant spectral diffusion, especially in nanostructures[49]. Moreover, most solid-state emitters appear at random 
Chapter 7: An integrated diamond nanophotonics platform for quantum optical networks

positions, making the realization of scalable devices with multiple emitters difficult $[204,154]$.

\subsection{Diamond platform for quantum nanophoton- ics.}

Our approach uses negatively-charged silicon-vacancy (SiV) color centers[58] integrated into diamond nanophotonic devices. SiV centers in high-quality diamond crystals show nearly lifetime-broadened optical transitions with an inhomogeneous (ensemble) distribution on the order of the lifetime-broadened linewidth[127]. These properties arise from the inversion symmetry of the $\mathrm{SiV}$ center which protects the optical transitions from electric field noise in the environment[142, 205].

The stable quantum emitters are integrated into one-dimensional diamond waveguides and photonic-crystal cavities with small mode volumes $(V)$ and large quality factors $(Q)$. These nanophotonic devices are fabricated using angled reactive-ion etching to scalably create free-standing single-mode structures starting from bulk diamond[195]. The fabrication process is described in Appendix D. As an example, Figs. 7.1C and D.3 show structures consisting of a notch for free space-waveguide coupling (at $\sim 1 \%$ efficiency), a waveguide section on each side and a cavity (Fig. 7.1B). The measured cavity $Q=7200$ (500) is limited predominantly by decay to the waveguide, so the system has high transmission on resonance. We measure the cavity mode profile and infer $V \sim 2.5(\lambda / n)^{3}$ using a uniform, high density SiV ensemble (Fig. D.4).

To obtain optimal coupling between an individual $\mathrm{SiV}$ and the cavity mode, we 

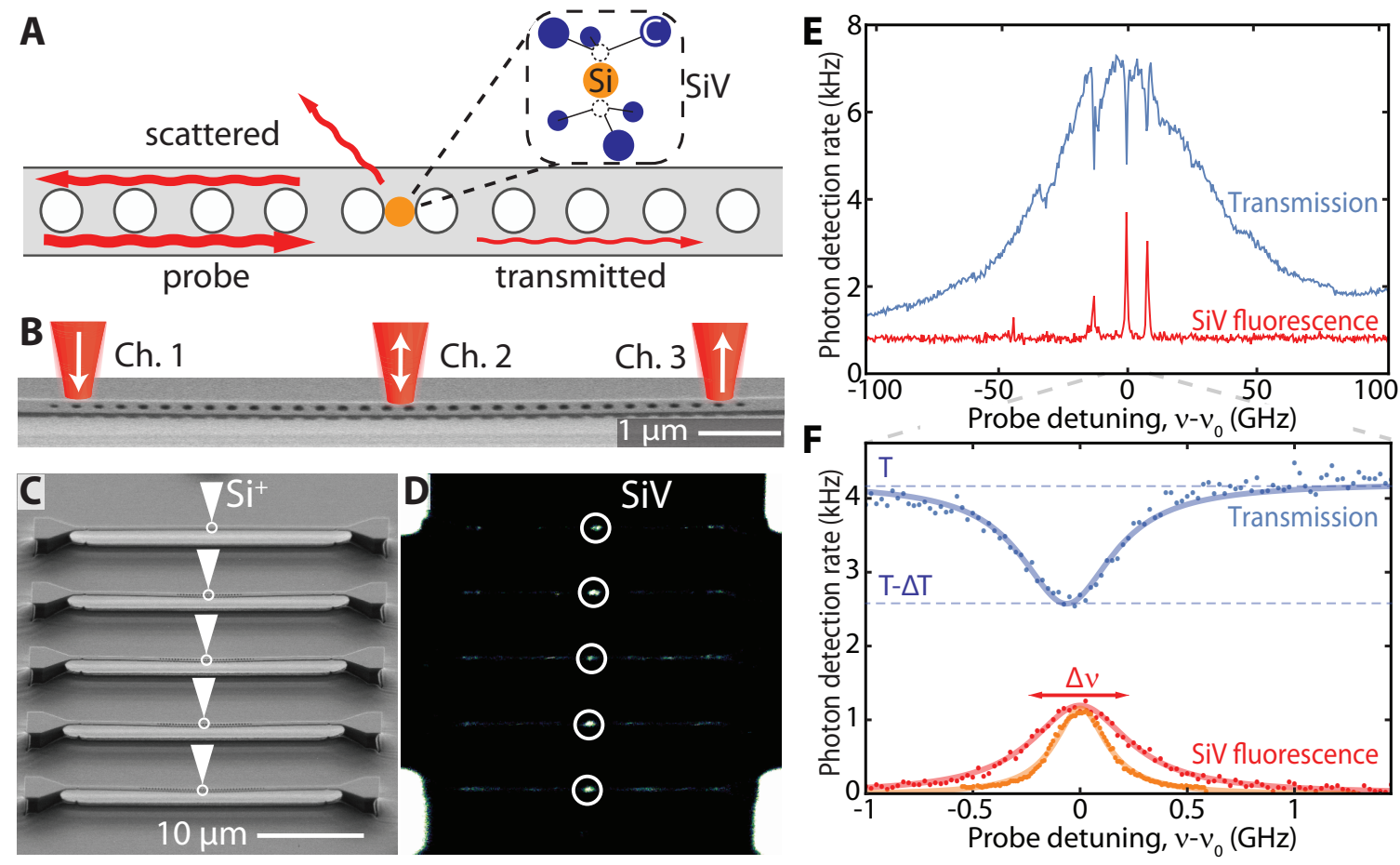

Figure 7.1: Positioning and strong coupling of SiVs in diamond cavities. (A) Schematic of an $\mathrm{SiV}$ center in a diamond photonic crystal cavity. (B) Scanning electron micrograph (SEM) of a cavity. (C) SEM of five cavities fabricated out of undoped diamond. After fabrication, SiV centers are deterministically positioned at the center of each cavity using focused $\mathrm{Si}^{+}$ion beam implantation. (D) $\mathrm{SiV}$ fluorescence is detected at the center of each nanocavity shown in (C). (E) Measured cavity transmission (blue, Ch. 3) and SiV scattered fluorescence (red, Ch. 2) spectrum. Three $\mathrm{SiV}$ centers are coupled to the cavity and each results in suppressed transmission. (F) Strong extinction, $\Delta T / T=38(3) \%$, of probe transmission from a single SiV center. Optical transition linewidths $\Delta \nu$ are measured with the cavity detuned (orange) and on resonance (red, count rate offset by $-150 \mathrm{~Hz}$ and multiplied by 3.3) with the $\mathrm{SiV}$ transition. 
Chapter 7: An integrated diamond nanophotonics platform for quantum optical networks

use a focused ion beam to implant $\mathrm{Si}^{+}$ions at the center of the cavities as illustrated in Fig. 7.1C. To form SiVs and mitigate crystal damage from fabrication and implantation, we subsequently anneal the sample at $1200{ }^{\circ} \mathrm{C}$ in vacuum[205]. This targeted implantation technique enables positioning of emitters inside the cavity with close to $40 \mathrm{~nm}$ precision in all three dimensions and control over the isotope and average number of implanted $\mathrm{Si}^{+}$ions. We fabricate $\sim 2000 \mathrm{SiV}$-cavity nodes on a single diamond sample with optimal spatial alignment. For example, Fig. 7.1D shows fluorescence from the array of cavities implanted with $\mathrm{Si}^{+}$ions in Fig. 7.1C. SiV fluorescence is detected at the center of each cavity, demonstrating high-yield creation of SiV-cavity nodes. The number of $\mathrm{SiVs}$ created varies based on the number of implanted ions and the $\sim 2 \%$ conversion yield from $\mathrm{Si}^{+}$to $\mathrm{SiV}$. For our experiments, we create an average of $\sim 5 \mathrm{SiVs}$ per cavity. Because each $\mathrm{SiV}$ can be resolved in the frequency domain, device creation is nearly deterministic: most $\mathrm{SiV}$-cavity nodes can be used for the experiments described below.

We characterize the coupled SiV-cavity system at $4 \mathrm{~K}$ using the setup described in Appendix D. As shown in Figs. 7.1B and D.1, three optical beams are focused on the nanostructure to excite the waveguide mode (Channel 1), to detect fluorescence scattering and to control the $\mathrm{SiV}$ (Channel 2) and to detect transmission (Channel 3). In subsequent experiments (Figs. 7.4 and 7.5), efficient collection with a tapered optical fiber is employed. We scan the frequency $\nu$ of the weak excitation laser across the $\mathrm{SiV}$ resonance $\nu_{0}=406.706 \mathrm{THz}$ and monitor the transmitted and scattered field intensities (Fig. 7.1E). We observe three fluorescence peaks in Channel 2 from three $\mathrm{SiV}$ centers in a single cavity (red curve in Fig. 7.1E). At the same time, within 
Chapter 7: An integrated diamond nanophotonics platform for quantum optical networks

the broad cavity transmission spectrum measured in Channel 3, each of these three resonances results in strong extinction of the cavity transmission indicating that all three SiVs couple to the cavity mode.

The strength of the SiV-cavity coupling is evaluated using the data in Fig. 7.1F. When the cavity is off-resonant with the emitter, the $\mathrm{SiV}$ transition linewidth is $\Delta \nu=298(5) \mathrm{MHz}$ (orange curve) and the excited state lifetime is $\tau_{e}=1.8(1) \mathrm{ns}$. This is close to the lifetime-broadening limit of $90 \mathrm{MHz}$ with additional nonradiative broadening likely due to a combination of finite-temperature effects[181] and residual spectral diffusion[205]. When the cavity is tuned into resonance, the transition is radiatively broadened to $\Delta \nu=590(30) \mathrm{MHz}$ (red curve) with a corresponding measured reduction in lifetime $\tau_{e}=0.6(1) \mathrm{ns}$ (limited by detection bandwidth). At the same time, we find that a single SiV results in $38(3) \%$ extinction of the probe field in transmission (Fig. 7.1F, blue curve). Based on the radiative broadening shown in Fig. 7.1F, we infer a cooperativity of $C=4 g^{2} / \kappa \gamma=1.0(1)$ for the SiV-cavity system with cavity QED parameters $\{g, \kappa, \gamma\} / 2 \pi=\{2.1,57,0.30\} \mathrm{GHz}$ where $g$ is the single-photon Rabi frequency, $\kappa$ is the cavity intensity decay rate and $\gamma$ is the $\mathrm{SiV}$ optical transition linewidth.

\subsection{Quantum-optical switch based on a single SiV center}

The coupled emitter-cavity system can be used to create strong interactions between single photons and achieve single-photon nonlinearities[206,198]. To probe the 
Chapter 7: An integrated diamond nanophotonics platform for quantum optical networks
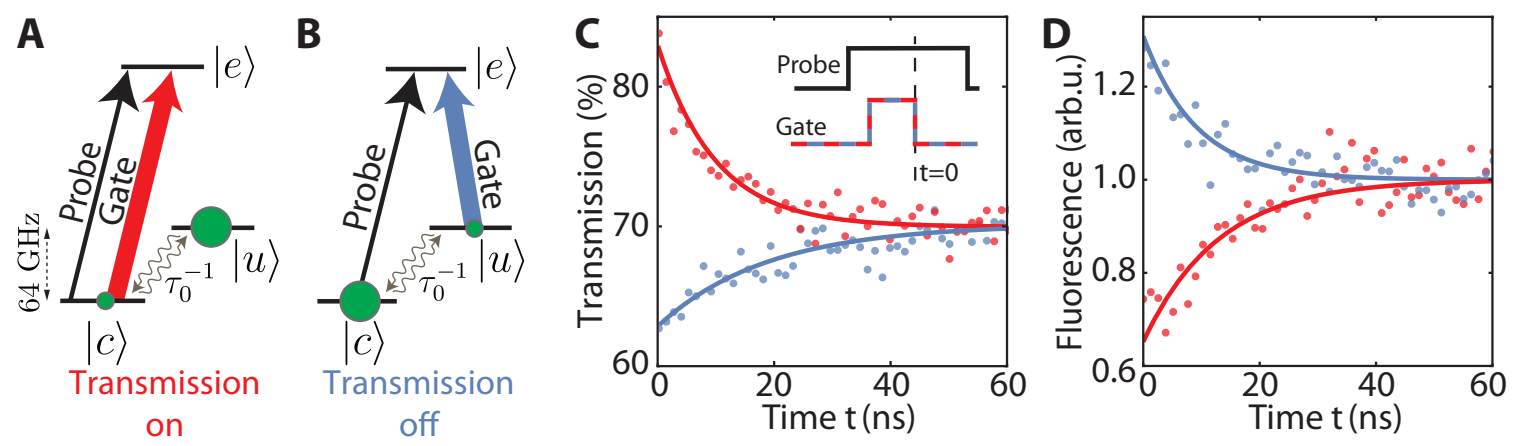

Figure 7.2: An all-optical switch using a single $\mathrm{SiV}$. The transmission of a probe field is modulated using a gate pulse that optically pumps the $\mathrm{SiV}$ to state $|u\rangle(\mathrm{A})$ or $|c\rangle(\mathrm{B})$. (C) Probe field transmission measured after the initialization gate pulse. Initialization in state $|u\rangle(|c\rangle)$ results in increased (suppressed) transmission, and (D) suppressed (increased) fluorescence.

nonlinear response of the $\mathrm{SiV}$-cavity system, we repeat the transmission and linewidth measurements of Fig. 7.1F at increasing probe intensities. As expected [207, 206], we find that the system saturates at a level less than a single photon per Purcell-enhanced excited-state lifetime (Fig. 7.3A), resulting in power broadening in fluorescence $(\Delta \nu)$ and reduced extinction in transmission $(\Delta T / T)$.

We realize an all-optical switch with memory by optically controlling the metastable orbital states[62, 61, 208] of a single SiV (Fig. 7.2). Specifically, we use a $30 \mathrm{~ns}$ long gate pulse to optically pump the $\mathrm{SiV}$ to an orbital state that is uncoupled $(|u\rangle$, Fig. 7.2A) or coupled $(|c\rangle$, Fig. $7.2 \mathrm{~B})$ to a weak probe field resonant with the cavity. The response of the system to the probe field after the gate pulse is monitored both in transmission (Fig. 7.2C) and fluorescence (Fig. 7.2D). If the gate pulse initializes the system in state $|c\rangle$ (blue curves), the transmission is reduced while the fluorescence scattering is increased. Initializing the system in state $|u\rangle$ (red curves) results in increased transmission and reduced fluorescence scattering. The observed modulation 
Chapter 7: An integrated diamond nanophotonics platform for quantum optical networks

demonstrates switching of a weak probe pulse by a classical gate pulse. The switch memory time is limited by a thermal phonon relaxation process between $|c\rangle$ and $|u\rangle$ that depolarizes the system over $\tau_{0} \sim 10 \mathrm{~ns}$ at $4 \mathrm{~K}[181]$.

To investigate these processes at the single-photon level, we resonantly excite the SiV-cavity system with a weak coherent light and measure photon statistics of the scattered and transmitted fields. To this end, scattered and transmitted light are each split to two detectors (Fig. D.1), allowing us to measure normalized intensity autocorrelations for the scattered $\left(g_{S S}^{(2)}(\tau)\right.$, Fig. 7.3B $)$ and transmitted $\left(g_{T T}^{(2)}(\tau)\right.$, Fig. 7.3C) fields as well as cross-correlations between the two channels $\left(g_{S T}^{(2)}(\tau)\right.$, Fig. 7.3D). At short timescales determined by the excited state lifetime $\tau_{e}$, we observe strong antibunching of photons scattered by the $\operatorname{SiV}\left(g_{S S}^{(2)}(0)=0.15(4)\right)$, consistent with scattering from a single emitter. In transmission, the photons are strongly bunched with $g_{T T}^{(2)}(0)=1.50(5)$. This photon bunching in transmission results from the interference between the weak probe field and the anti-bunched resonant scattering from the $\mathrm{SiV}$. The destructive interference for single photons yields preferential transmission of photon pairs and is a direct indication of nonlinear response at the single-photon level[207, 206]. In other words, a single photon in an optical pulse switches a second photon, and the system acts as a photon number router where single photons are scattered while photon pairs are preferentially transmitted. Finally, both bunching $\left(g_{S T}^{(2)}(0)=1.16(5)\right)$ and anti-bunching are observed for scattering-transmission cross-correlations at fast and slow timescales respectively.

To understand the system saturation and switching responses in Figs. 7.2 and 7.3, we model the quantum dynamics of the SiV-cavity system using the cavity QED 
A

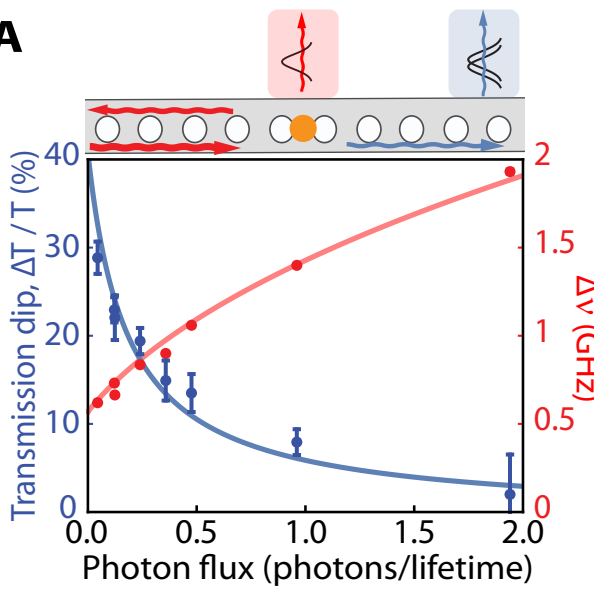

C

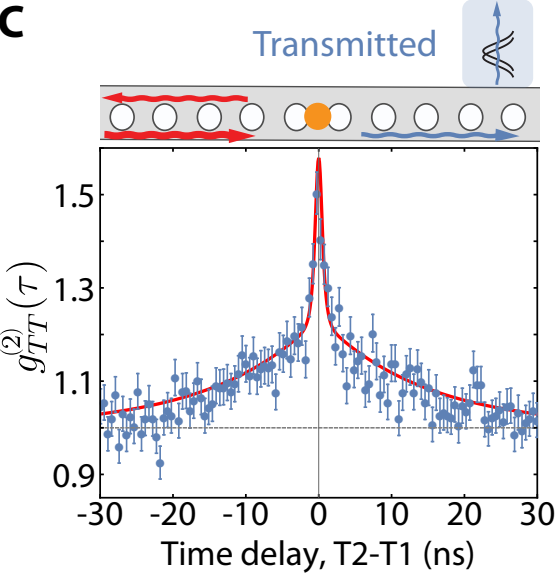

B scattered $\hat{\&}$
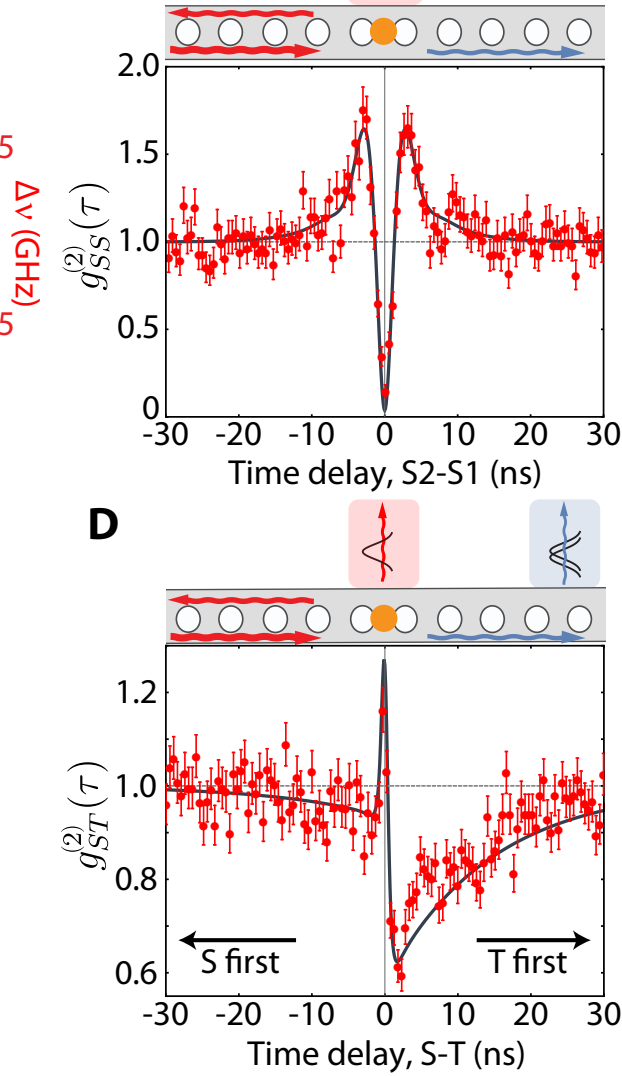

Figure 7.3: Single-photon switching. (A) Cavity transmission and SiV transition linewidth measured at different probe intensities. (B, C) Intensity autocorrelations of the scattered (fluorescence) and transmitted fields. The scattered field shows antibunching (B), while the transmitted photons are bunched with an increased contribution from photon pairs (C). (D) Intensity cross-correlation between the scattered and transmitted fields. $g_{S S}^{(2)}$ is measured under different conditions with above saturation excitation. 
Chapter 7: An integrated diamond nanophotonics platform for quantum optical networks

parameters measured in Fig. 7.1 and a three-level model of the SiV (Figs. 7.2 and D.7). This model is described in detail in Appendix D. The results of our calculation are in excellent agreement with our observations (solid curves in Figs. 7.1-7.3). Specifically, the presence of a second metastable state, $|u\rangle$, reduces the extinction in linear transmission (Fig. 7.1F) and affects the nonlinear saturation response[206]. The metastable state $|u\rangle$ also causes both slow dynamics in photon correlation measurements (Fig. 7.3) at the metastable orbital relaxation timescale of $\tau_{0}$ as well as an asymmetry in cross-correlations. In these measurements, the detection of a transmitted (scattered) photon preferentially prepares the $\mathrm{SiV}$ in state $|u\rangle(|c\rangle)$, resulting in enhanced (reduced) transmission and reduced (enhanced) scattering for $\tau_{0}$.

\subsection{Tunable single-photon source using Raman tran- sitions.}

A key challenge for building scalable quantum networks using solid-state emitters is the spectral inhomogeneity of their optical transitions. Although the inhomogeneous broadening of $\mathrm{SiVs}$ is suppressed by inversion symmetry, SiVs inside nanostructures still display a substantial inhomogeneous distribution (seen in Fig. 7.1E) due to residual strain from fabrication[205]. To mitigate this effect, we use Raman transitions between the metastable orbital states of $\mathrm{SiV}$ centers. When a single $\mathrm{SiV}$ is excited from the state $|u\rangle$ at a detuning $\Delta$ (Fig. 4B), the emission spectrum includes a spontaneous component at frequency $\nu_{e c}$ and a Raman component at frequency $\nu_{e c}-\Delta$ that is tunable by choosing $\Delta$. 

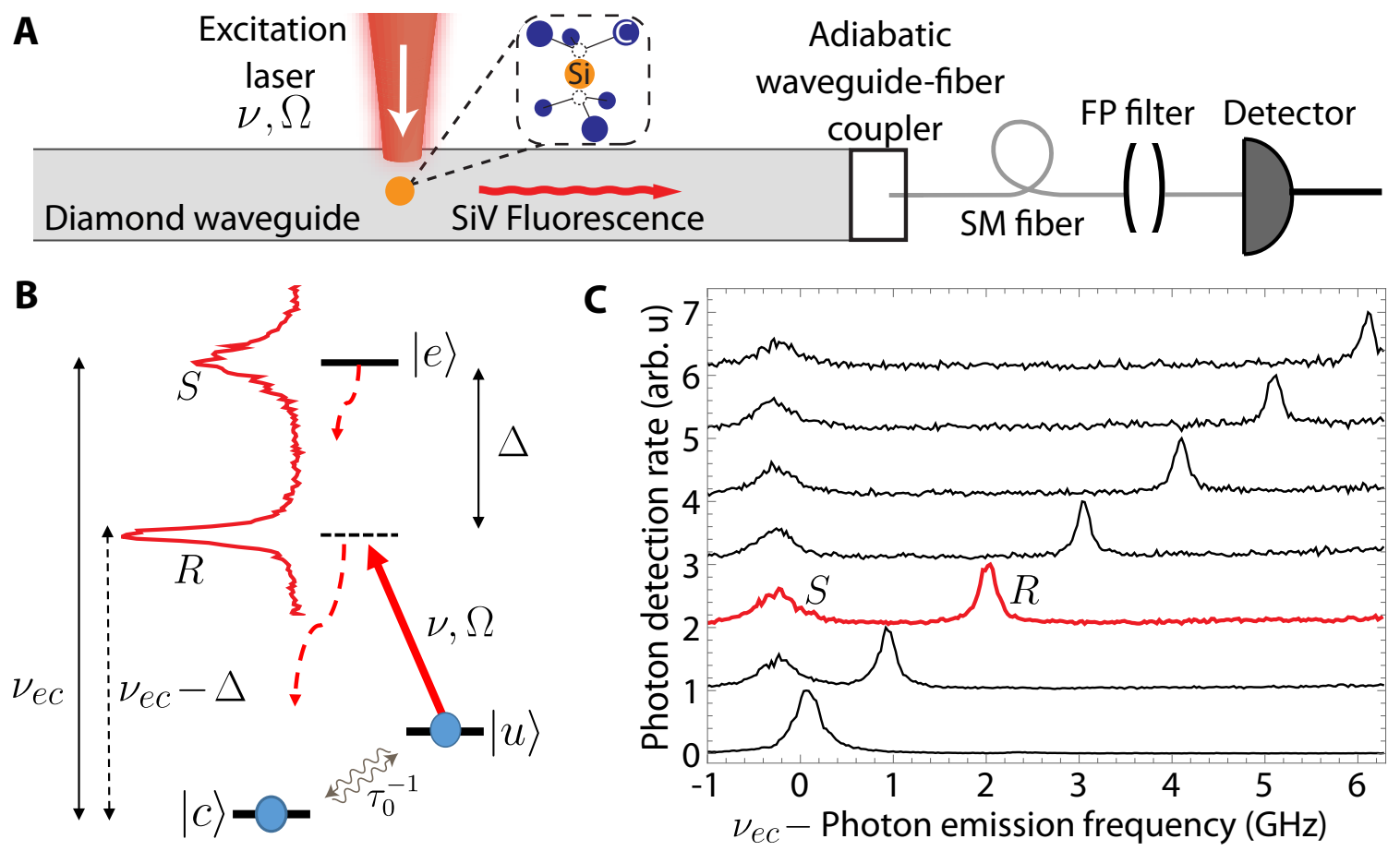

Figure 7.4: Spectrally-tunable single-photons using Raman transitions. (A) Photons scattered by a single SiV into a diamond waveguide are efficiently coupled to a singlemode (SM) fiber. A scanning Fabry-Perot (FP) cavity is used to measure emission spectra. (B) Under excitation at a detuning $\Delta$, the emission spectrum contains spontaneous emission (labeled $S$ ) at frequency $\nu_{e c}$ and narrow Raman emission $(R)$ at frequency $\nu_{e c}-\Delta$. (C) $\Delta$ is varied from 0 to $6 \mathrm{GHz}$ in steps of $1 \mathrm{GHz}$ and a corresponding tuning of the Raman emission frequency is observed. The red curves in (B) and (C) are the same data. 
Chapter 7: An integrated diamond nanophotonics platform for quantum optical networks

Tunable single-photon emission is experimentally realized by implanting SiVs inside a one-dimensional diamond waveguide and continuously exciting the emitters from free space (Fig. 7.4A). The fluorescence scattering into the diamond waveguide is coupled to a tapered single-mode fiber with $\geq 70 \%$ efficiency using adiabatic mode transfer[134, 209, 210]. As we change the excitation frequency from near-resonance to a detuning of $\Delta=6 \mathrm{GHz}$, we observe a corresponding tuning of the Raman emission frequency $\nu_{e c}-\Delta$ while the spontaneous emission frequency remains nearly fixed at $\nu_{e c}$ up to an AC Stark shift (Figs. 7.4C and D.8).

The Raman linewidth can be controlled by both the detuning and the power of the driving laser, and is ultimately limited by the ground state coherence between states $|u\rangle$ and $|c\rangle$. At large detunings and low power, we measure a subnatural Raman linewidth of less than $30 \mathrm{MHz}$ (Fig. D.8). The nonclassical nature of the Raman emission is demonstrated via photon correlation measurements. The Raman photons from a single $\mathrm{SiV}$ are antibunched with $g_{\text {single }}^{(2)}(0)=0.16$ (3) (orange curve in Fig. 7.5D) close to the ideal limit $g_{\text {single }}^{(2)}(0)=0$. For the continuous excitation used here, we detect Raman photons at a rate of $\sim 15 \mathrm{kHz}$ from a single SiV. After a Raman scattering event, the SiV cannot scatter a second photon within the metastable orbital state relaxation timescale $\tau_{0}$, limiting the Raman emission rate. This rate can be improved using a pulsed excitation scheme where the $\mathrm{SiV}$ is first prepared in state $|u\rangle$ via optical pumping and subsequently excited with a pulse of desired shape and duration. Unlike previous demonstrations of Raman tuning of solid-state quantum emitters $[211,212]$, the tuning range demonstrated here is comparable to the inhomogeneous distribution of the $\mathrm{SiV}$ ensemble and can thus be used to tune 
Chapter 7: An integrated diamond nanophotonics platform for quantum optical networks

pairs of $\mathrm{SiV}$ centers into resonance.

\subsection{Entanglement of two $\mathrm{SiV}$ centers in a nanopho- tonic waveguide}

Quantum entanglement is an essential ingredient in quantum networks[8]. Although optical photons were recently used to entangle solid-state qubits over longdistances[66, 213], optically-mediated entanglement of solid-state qubits in a single nanophotonic device has not yet been observed.

Motivated by the proposals for probabilistic entanglement generation based on interference of indistinguishable photons[214], we use two $\mathrm{SiV}$ centers inside a diamond waveguide (Fig. 7.5A), continuously excite each $\mathrm{SiV}$ on the $|u\rangle \rightarrow|e\rangle$ transition with a separate laser, and measure photon correlations in the waveguide mode. If the Raman transitions of the two SiVs are not tuned into resonance, the photons are distinguishable, resulting in the measured $g_{\text {dist }}^{(2)}(0)=0.63(3)$ (blue curve in Fig. 7.5D) close to the conventional limit associated with two single photon emitters $g_{\text {dist }}^{(2)}(0)=0.5$ [142]. Alternatively, if the Raman transitions of the two SiVs are tuned instead into resonance with each other, an interference feature is observed in photon correlations around zero time delay with $g_{\text {ind }}^{(2)}(0)=0.98$ (5) (red curve in Fig. 7.5D).

These results can be understood by considering the level diagrams in Figs. 7.5B and 7.5C involving the $\mathrm{SiV}$ metastable states $|u\rangle$ and $|c\rangle[215,202]$. Photon correlation measurements probe the conditional dynamics of the two SiVs starting in state $|u u\rangle$. In this state, each $\mathrm{SiV}$ scatters Raman single photons to the waveguide at a rate $\Gamma_{1 D}$. 
Chapter 7: An integrated diamond nanophotonics platform for quantum optical networks

However, when the Raman transitions of the two SiVs are tuned into resonance with each other, it is fundamentally impossible to distinguish which of the two emitters produced a waveguide photon. Thus, emission of an indistinguishable single photon leaves the two $\mathrm{SiVs}$ prepared in the entangled state $|B\rangle=\left(|c u\rangle+e^{i \phi}|u c\rangle\right) / \sqrt{2}[214]$ (Fig. 7.5B), where $\phi$ is set by the propagation phase between emitters spaced by $\Delta L$ and the relative phase of the driving lasers which is constant in each experimental run (Fig. D.9). This state is a two-atom superradiant Dicke state with respect to the waveguide mode, independent of the value of $\Delta L[215]$. This implies that although there is only a single excitation stored in the state $|B\rangle$, it will scatter Raman photons at a rate $2 \Gamma_{1 D}$ that is twice the scattering rate of a single emitter. This enhanced emission rate into the waveguide mode results in the experimentally observed interference peak at short time delays (Fig. 7.5D) and is a signature of entanglement. Our measured value of $g_{\text {ind }}^{(2)}(0)=0.98(5)$ is close to the ideal limit where the factor of two enhancement in the emission from the entangled state $|B\rangle$ yields $g_{i n d}^{(2)}(0)=1$.

The visibility of the interference signal in photon correlation measurements in Fig. $7.5 \mathrm{D}$ can be used to evaluate a lower bound on the conditional entanglement fidelity $F=\langle B|\rho| B\rangle$. For experimental runs where we detect a photon coincidence within the interference window (rate $\sim 0.5 \mathrm{~Hz}$ ), we find that the two SiVs were in an entangled state with $F \geq 82(7) \%$ after the emission of the first photon. This conditional fidelity is primarily limited by laser leakage and scattering from nearby SiVs that yield false detection events. Our measurements also demonstrate entanglement generation (rate $\sim 30 \mathrm{kHz}$ ) after a single Raman photon emission event. As discussed in Appendix D, using photon correlation data and steady state populations of $\mathrm{SiV}$ 


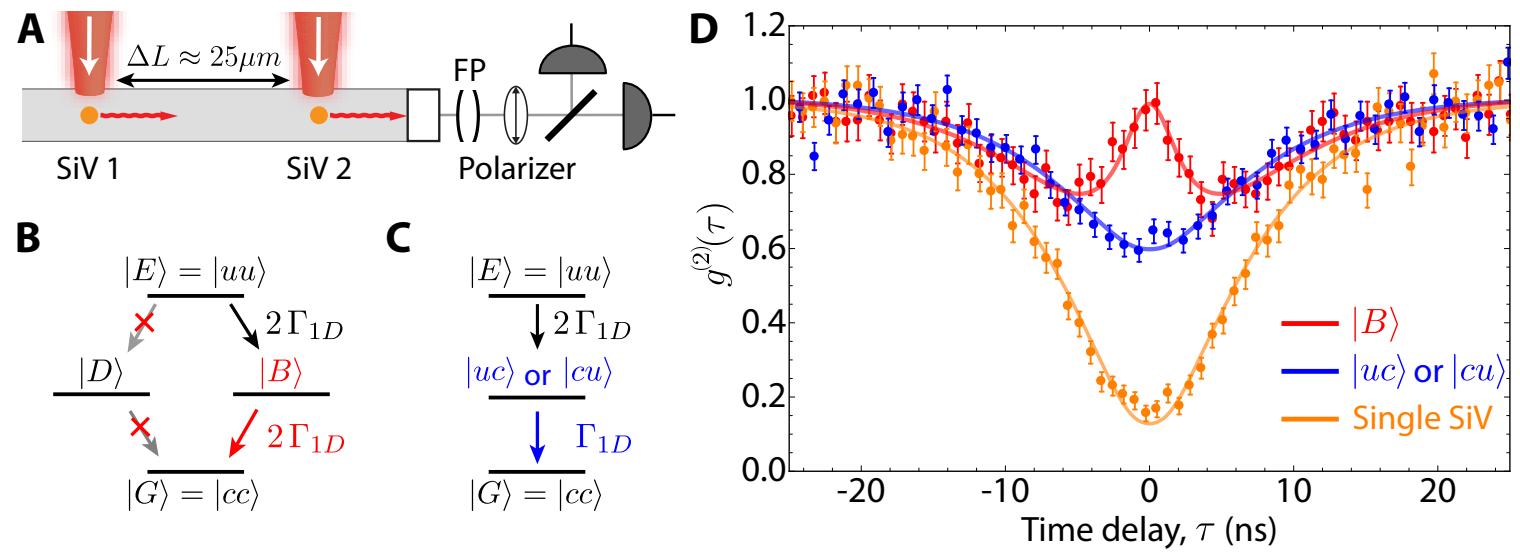

Figure 7.5: Quantum interference and two-SiV entanglement in a nanophotonic waveguide. (A) Photons scattered by two SiV centers into a diamond waveguide are detected after a polarizer. (B) After emission of an indistinguishable photon, the two SiVs are in the entangled state $|B\rangle$ which decays at the collectively enhanced rate of $2 \Gamma_{1 D}$ into the waveguide. (C) After emission of a distinguishable photon, the two SiVs are in a classical mixture of states $|u c\rangle$ and $|c u\rangle$ which decays at a rate $\Gamma_{1 D}$ into the waveguide. (D) Intensity autocorrelations for the waveguide photons. Exciting only a single $\mathrm{SiV}$ yields $g_{\text {single }}^{(2)}(0)=0.16$ (3) for SiV1 (orange data), and $g_{\text {single }}^{(2)}(0)=0.16$ (2) for SiV2. Blue: Both SiVs excited; Raman photons are spectrally distinguishable. Red: Both SiVs excited; Raman photons are tuned to be indistinguishable. The observed contrast between the blue and the red curves at $g^{(2)}(0)$ is due to the collectively enhanced decay of $|B\rangle$. The solid curves are fits to a model described in Appendix D. 
Chapter 7: An integrated diamond nanophotonics platform for quantum optical networks

orbital states, we find that a single photon emission results in an entangled state with positive concurrence $\mathcal{C}>0.090(0.024)$, which is limited by imperfect initialization in state $|u u\rangle$. The width of the interference signal in Fig. 7.5D can be used to extract a lifetime $T_{2}^{*} \approx 2.5 \mathrm{~ns}$ of the entangled state $|B\rangle$. This lifetime is mainly limited by imperfect spectral tuning of the two Raman photons from the two SiVs, resulting in a relative frequency detuning $\delta$. In this regime, emission of a first photon results in a state $|\psi(\tau)\rangle=\left(|c u\rangle+e^{i(\phi-2 \pi \delta \tau)}|u c\rangle\right) / \sqrt{2}$ that oscillates at frequency $\delta$ between states $|B\rangle$ and the subradiant state $|D\rangle=\left(|c u\rangle-e^{i \phi}|u c\rangle\right) / \sqrt{2}$. Since $|D\rangle$ does not couple to the waveguide mode due to destructive interference, fluctuations in $\delta$ over different realizations result in decay of the collectively enhanced signal (central peak in red curve in Fig. 7.5D).

Note that in our experiment, the photon propagation time is longer than $T_{2}^{*}$ and the entangled state dephases before it can be heralded by detection of the first photon. To generate useful heralded entanglement with high fidelity[214, 213], a pulsed excitation scheme can be employed where the two SiVs are optically initialized in state $|u u\rangle$ and excited by short pulses. The Raman emission frequencies can be further stabilized using a narrowband reference cavity to extend the lifetime of the entangled state.

\subsection{Outlook}

The performance of these quantum nanophotonic devices can be improved in several ways. Control over the $\mathrm{SiV}$ orbital states is limited by the occupation of $\sim 50 \mathrm{GHz}$ phonons at $4 \mathrm{~K}$, which causes relaxation between the metastable orbital states $|u\rangle$ and 
Chapter 7: An integrated diamond nanophotonics platform for quantum optical networks

$|c\rangle$ and limits their coherence times to less than 50 ns. Phonon relaxation should be significantly suppressed by operating at temperatures below $300 \mathrm{mK}$ or engineering the phononic density of states to enable millisecond-long coherence times[181]. Even longer-lived quantum memories can potentially be obtained by storing the qubit in the ${ }^{29} \mathrm{Si}$ nuclear $\operatorname{spin}[61]$ which is only weakly coupled to the environment[92]. Furthermore, the demonstrated cooperativity in our nanocavity experiment is lower than the theoretical estimate based on an ideal two-level emitter optimally positioned in a cavity. The discrepancy is due to a combination of factors including imperfect spatial and polarization alignment, phonon broadening[181], finite quantum efficiency[154], the branching ratio of the transition and residual spectral diffusion[205]. These imperfections also limit the collection efficiencies obtained in our waveguide experiments. Despite uncertainties in individual contributions which are discussed in Appendix $\mathrm{D}$, operation at lower temperatures and improved cavity designs with higher $Q / V$ ratios should enable spin-photon interfaces with high cooperativity $C \gg 1$. Furthermore, the efficient fiber-diamond waveguide coupling can be improved to exceed $95 \%$ efficiency [209].

Our work demonstrates key ingredients required for realizing integrated quantum network nodes and opens up new possibilities for realizing large-scale systems involving multiple emitters strongly interacting via photons. Our fabrication approach can be used to create systems involving many coupled emitters per cavity as well as arrays of multiple atom-cavity nodes. Such a system can be used to implement entanglement generation, quantum memories and quantum gates for either photonic or spin qubits, paving the way for the realization of scalable quantum networks[8, 198]. 


\section{Chapter 8}

\section{Conclusions and outlook}

In this thesis, we presented progress on coherently interfacing solid-state spins with optical photons. The results of Chapter 2 have already been used as a basis for elementary quantum-network[8] demonstrations based on NV centers, including the first demonstration of a loophole-free Bell inequality test[66], and entanglement distillation between remote stationary qubits[216]. The scalability of these experiments to multiple quantum network nodes is currently limited by the low coherent photon generation probability from NV centers. In Chapters 4 and 7 , we showed that $\mathrm{SiV}$ centers can address this challenge by demonstrating a deterministic spin-photon interface. In Chapter 5, we showed that spin coherence of $\mathrm{SiV}$ centers is limited by acoustic phonons, and the spin coherence can be extended by operating at low temperatures. These results establish the SiV center as one of the leading solid-state candidates for a quantum network node. In what follows, we give examples of possible future directions towards realizing quantum-network nodes based on color centers. 


\subsection{Long-lived SiV spin coherence at low temper-}

\section{atures}

Motivated by the predictions in Chapter 5, we set up a confocal microscope that operates inside a cryogen-free dilution refrigerator (BlueFors BF-LD250). With this system, we can carry out optical experiments with single emitters while maintaining a base temperature of $20 \mathrm{mK}$. The preliminary investigations of $\mathrm{SiV}$ centers at these low temperatures indicate that the phonon relaxation mechanisms that limit spin coherence are significantly suppressed below $500 \mathrm{mK}$. Using coherent population trapping (CPT) techniques [61, 62], we observed spin resonances as narrow as $400 \mathrm{kHz}$. The linewidth of the $\mathrm{SiV}$ spin resonance was found to be similar to the narrowest $\mathrm{CPT}$ linewidths observed in NV centers and is likely limited by the slowly evolving nuclear spin bath $[217,218,219]$. This suggests that significantly longer spin coherences could be observed using dynamical decoupling techniques[220]. These ongoing efforts could enable the first demonstration of a solid-state spin with both long spin coherence and a deterministic interface to optical photons.

\subsection{Photon-mediated two-qubit gates}

In Chapter 7, we presented key ingredients required to realize photon-mediated two-qubit gates between two SiV centers in a cavity. When the Raman transitions of two $\mathrm{SiV}$ centers in a cavity are tuned into resonance, the two emitters will interact via exchange of virtual photons[14]. Such exchange interactions can be used to realize gates between pairs of $\mathrm{SiV}$ centers inside a nanophotonic structure[221]. The fidelity of 
these gates will depend on the system cooperativity and the ability to independently

tune transitions of different $\mathrm{SiV}$ centers into resonance. By improving the cavity design in Chapter 7, it seems possible to achieve large enough cooperativities $(C \sim$ $5-10)$ to observe coherent two-SiV interactions. The SiVs can be tuned either using strain[222] or using Raman transitions as shown in Chapter 7.

\subsection{Study of new color centers}

A major challenge in quantum information science is to develop a solid-state quantum-network node with optical and spin transitions that are robust against sources of decoherence. Based on the detailed understanding of the electronic structures of NV and $\mathrm{SiV}$ centers developed over the past few years, we can identify the desired symmetry and electronic properties of the ideal color center to build a quantum-network node.

Among hundreds of color centers in diamond [42], only the NV and SiV centers have been studied in enough detail to reproducibly demonstrate coherent spin and optical control. However, each of these two systems has its strengths and weaknesses. Specifically, the NV centers have a robust spin transition with a long spin coherence up to room temperature. However, the NV centers' optical transitions are weak and susceptible to electric field noise. In comparison, $\mathrm{SiV}$ centers have robust optical transitions that maintain coherence in nanostructures. However, the SiV spin transitions are highly susceptible to phonons and temperatures below $500 \mathrm{mK}$ are required to obtain long spin coherence.

In this thesis, we described the key properties that resulted in these weaknesses. 
Based on this understanding, we can identify two key electronic and symmetry properties that will lead to simultaneously robust spin and optical transitions in a color center: (i) Robust optical transitions require a large transition dipole moment (to maximize radiative decay rate) and a vanishing static electric dipole moment (to minimize dephasing due to electric field noise). Chapter 4 has shown that these properties can be simultaneously be achieved in defects with inversion symmetry. (ii) To achieve robust spin-transitions at high temperatures, the system should not have an orbital degeneracy in its ground state, as such degeneracies result in strong interactions with the phonon bath (as shown in Chapters 3 and 5). For $e$-orbitals with double degeneracy, this can be achieved by having a spin-triplet and orbital-singlet ground state like the negatively charged NV center or the neutral SiV center [148]. Alternatively, $\mathrm{S}=1 / 2$ systems with non-degenerate orbitals ( $a$ - or s-like orbitals) in their ground state configuration also result in decoupling from the phonon bath. Examples of such systems include donor-spin qubits in silicon[223, 224].

We note that while there have been previous attempts to lay out such search criteria for new color centers [138], these efforts have not taken into consideration the coherence of the optical transitions. Despite exciting progress in identifying color centers in other wide-bandgap crystals such as silicon carbide and zinc oxide[22], these crystals do not contain inversion centers. This consideration makes diamond a unique wide-bandgap host for color centers with inversion symmetry and robust optical transitions. Density functional theory (DFT) calculations of defects in diamond indicate that a large family of color centers have inversion symmetry like the $\mathrm{SiV}$ center, and the right spin-triplet configuration to achieve long spin coherence[225]. 
Motivated by these studies, we investigated the optical properties of the recently discovered negatively charged germanium-vacancy $(\mathrm{GeV})$ center defect which has the same symmetry properties with the $\operatorname{SiV}[226,227,228,229]$. In Ref. [230], we have recently shown the $\mathrm{GeV}$ optical properties to be even better compared with the $\mathrm{SiV}$ center, demonstrating the potential of the large number of uninvestigated centers in diamond. 


\section{Appendix A}

\section{Supporting material for Chapter 3}

\section{A.1 Optical Rabi oscillation decoherence measure- ment}

To measure the decoherence of optical Rabi oscillations, we apply the pulse sequence shown in Fig. A.1. The application of green light initializes the NV center to the negatively charged state by ionizing local charge traps in the diamond [88], which shifts the local electric field and, through the DC Stark effect, induces spectral diffusion of the NV center's optical transitions. We negate this spectral diffusion with a preselection stage that tests whether the NV center's transitions are resonant with the excitation lasers [102]. During the strong excitation pulse, we measure the detection times of photons in the phonon sideband (PSB) relative to the pulse beginning. The spontaneous emission rate into the PSB is instantaneously proportional to the

population in $\left|E_{\mathrm{x}}\right\rangle$, enabling us to measure directly the decoherence of Rabi oscilla- 
tions and decay via spontaneous emission after the end of the pulse. We repeat this procedure at many temperatures between $5.8 \mathrm{~K}$ and $24 \mathrm{~K}$. Typical results for three temperatures are shown in Fig. 3.2(a).

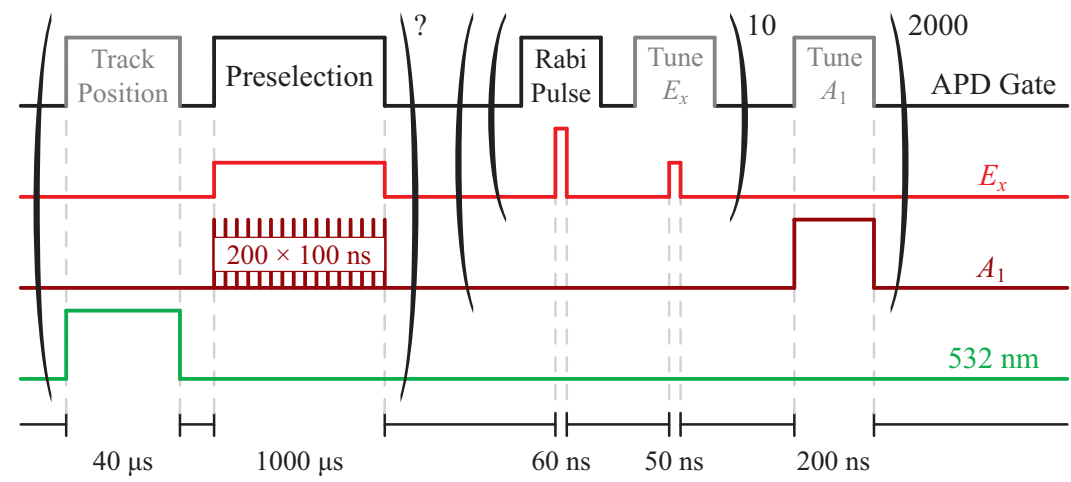

Figure A.1: The pulse sequence used to measure optical Rabi decoherence. In the first stage, we initialize the NV center into the negatively charged state and $|0\rangle$ electronic state with nonresonant excitation at $532 \mathrm{~nm}$, then we apply resonant excitation with reduced intensity/duty cycle to determine whether the NV center's transitions are on resonance. The first stage is repeated until the number of photons collected during the preselection period surpasses a specified threshold. In the second stage, we strongly excite the $|0\rangle-\left|\mathrm{E}_{\mathrm{x}}\right\rangle$ transition ten times before repumping on the $| \pm 1\rangle-\left|A_{1}\right\rangle$ transition. Every few minutes during the experiment, we compensate for slow drifts by measuring the PSB fluorescence during the periods shown in grey: we weakly excite the $|0\rangle-\left|E_{\mathrm{x}}\right\rangle$ transition to tune the excitation laser precisely, we excite the $| \pm 1\rangle-\left|A_{1}\right\rangle$ transition to tune the repump laser, and we count the photons emitted during nonresonant initialization to steer our optical path to track the NV center's position.

As described in the main text, we extract $\Gamma_{\text {Add }}$, the additional decoherence rate of the Rabi oscillations due to processes other than spontaneous emission to $|0\rangle$, from each experiment iteration. We fit the extracted values of $\Gamma_{\text {Add }}$ to

$$
\Gamma_{\text {Add }}(T)=A\left(T-T_{0}\right)^{5}+C
$$


to extract the fit constant values

$$
\begin{aligned}
A & =2 \pi \times(2.0 \pm 0.9) 10^{-5} \mathrm{MHz} / \mathrm{K}^{5} \\
T_{0} & =4.4 \pm 1.5 \mathrm{~K} \\
C & =2 \pi \times(0.08 \pm 0.56) \mathrm{MHz},
\end{aligned}
$$

where the uncertainties are the $95 \%$ confidence interval bounds on the fit parameters. The best fit and the 95\% confidence bands are shown in Fig. 2(a) in the main text. We also conducted this experiment repeatedly at $5.8 \mathrm{~K}$ to measure $\Gamma_{\text {Add }}=-0.34 \pm 1.87$ $\mathrm{MHz}$, where the uncertainty is given by twice the standard deviation of the extracted $\Gamma_{\text {Add }}$ values.

In order to extract a value for $\eta$, which parameterizes the electron-phonon coupling strength in our model of the ISC mechanism, we set $A$ equal to the $T^{5}$ coefficient of the $\left|E_{x}\right\rangle-\left|E_{y}\right\rangle$ mixing rate

$$
\Gamma_{\mathrm{Mix}}=\frac{64}{\pi} \hbar \alpha \eta^{2} k_{B}^{5} T^{5},
$$

where $\alpha=25.9$ is a numeric constant, that is calculated in Ref. [104]. We find $\eta=2 \pi \times(44.0 \pm 2.4) \mathrm{MHz} \mathrm{meV}^{-3}$. 


\section{A.2 Rabi decoherence analysis}

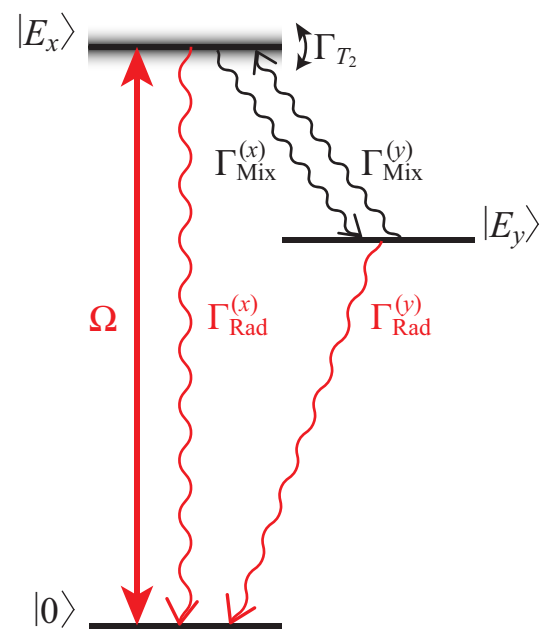

Figure A.2: The three-level system used to investigate population mixing between $\left|\mathrm{E}_{\mathrm{x}}\right\rangle$ and $\left|\mathrm{E}_{\mathrm{y}}\right\rangle$. The directional mixing rates $\Gamma_{\text {Mix }}^{(x)}$ and $\Gamma_{\text {Mix }}^{(y)}$, radiative decay rates $\Gamma_{\mathrm{Rad}}^{(x)}$ and $\Gamma_{\mathrm{Rad}}^{(y)}$, and decoherence rate $\Gamma_{T_{2}}$ on the $|0\rangle-\left|\mathrm{E}_{\mathrm{x}}\right\rangle$ transition are shown.

In order to extract the phonon-induced mixing rate from the Rabi decoherence data, we must understand how the various rates factor into the Rabi decoherence timescale. To that end, we solve the master equation in Lindblad form for the three level system shown in Fig. A.2. For the sake of generality, we label the two mixing rates and the two radiative decay rates separately according to the initial states of the respective processes.

We find that the emitted fluorescence $\left(\propto \rho_{x x}+\rho_{y y}\right)$ oscillates within an envelope given by

$$
g(t)=\frac{1}{2}\left(\mathrm{e}^{-t / \tau_{\text {Rabi }}}+A \mathrm{e}^{-t / \tau_{2}}+B\right)
$$

where the exponential timescales are

$$
\frac{1}{\tau_{\text {Rabi }}}=\frac{3}{4} \Gamma_{\text {Rad }}^{(x)}+\frac{1}{2}\left(\Gamma_{\text {Mix }}^{(x)}+\Gamma_{T_{2}}\right)
$$


and

$$
\frac{1}{\tau_{2}}=\frac{1}{2}\left(2 \Gamma_{\mathrm{Rad}}^{(y)}+\Gamma_{\mathrm{Mix}}^{(x)}+2 \Gamma_{\mathrm{Mix}}^{(y)}\right)
$$

and the coefficients are

$$
A=\frac{-\Gamma_{\text {Mix }}^{(x)}}{2 \Gamma_{\text {Rad }}^{(y)}+\Gamma_{\text {Mix }}^{(x)}+2 \Gamma_{\text {Mix }}^{(y)}}
$$

and

$$
B=\frac{2\left(\Gamma_{\mathrm{Rad}}^{(y)}+\Gamma_{\mathrm{Mix}}^{(x)}+\Gamma_{\mathrm{Mix}}^{(y)}\right)}{2 \Gamma_{\mathrm{Rad}}^{(y)}+\Gamma_{\text {Mix }}^{(x)}+2 \Gamma_{\text {Mix }}^{(y)}} .
$$

Because $\left|\mathrm{E}_{\mathrm{x}}\right\rangle$ and $\left|\mathrm{E}_{\mathrm{y}}\right\rangle$ are separated by $3.9 \mathrm{GHz} \ll k_{B} T / 2 \pi \hbar$, we assume that $\Gamma_{\text {Mix }}^{(x)}=\Gamma_{\text {Mix }}^{(y)}$, which sets an upper limit of $A \leq \frac{1}{3}$. We therefore neglect the term of $g(t)$ that decays on a timescale of $\tau_{2}$ and we set the measured Rabi oscillation decoherence timescale equal to $\tau_{\text {Rabi }}$. We rearrange Eq. A.5 to find

$$
\Gamma_{\text {Mix }}^{(x)}+\Gamma_{T_{2}}=2\left(\frac{1}{\tau_{\text {Rabi }}}-\frac{3}{4} \Gamma_{\text {Rad }}\right),
$$

which is reproduced with $\Gamma_{\text {Mix }}^{(x)}=\Gamma_{\text {Mix }}$ as Eq. 1 in the main text.

If we set $\Gamma_{\mathrm{Mix}}^{(x)}, \Gamma_{\mathrm{Mix}}^{(y)} \rightarrow 0$, then we recover the standard result [103]

$$
g^{\prime}(t)=\frac{1}{2}\left(1+\mathrm{e}^{-t / \tau_{\text {Rabi }}^{\prime}}\right)
$$

with

$$
\frac{1}{\tau_{\text {Rabi }}^{\prime}}=\frac{3}{4} \Gamma_{\text {Rad }}+\frac{1}{2} \Gamma_{T_{2}}
$$

\section{A.3 Fluorescence depolarization measurement}

ZPL photons emitted by decay from the $\left|\mathrm{E}_{\mathrm{x}}\right\rangle$ and $\left|\mathrm{E}_{\mathrm{y}}\right\rangle$ states have orthogonal linear polarizations in the plane orthogonal to the $\mathrm{N}-\mathrm{V}$ axis. Therefore, we can use a polarizer in the collection path to preferentially collect fluorescence from either 
transition while suppressing fluorescence from the other. This technique enables us to use depolarization of the NV center fluorescence to measure mixing of the electronic state population.

Our polarization selectivity, however, is not perfect. Because the $\mathrm{N}-\mathrm{V}$ axis lies along the [111] crystallographic axis but we collect fluorescence emitted primarily along the [100] axis, the two polarizations are not perfectly orthogonal in the lab frame. Additionally, dichroic filters in the optical path substantially rotate polarizations that are not aligned either vertically or horizontally. As a result, there is no perfect set of polarization settings for the experiment. Instead, we must balance our simultaneous needs to suppress fluorescence from the undesired transition, collect fluorescence from the desired transition efficiently, suppress reflections of the strong excitation pulse, and excite the $|0\rangle \rightarrow\left|E_{\mathrm{x}}\right\rangle$ transition efficiently.

We apply a pulse sequence similar to that shown in Fig. A.1, except that we now record the arrival times of photons emitted into the ZPL instead of the PSB. Also, we now apply a short ( $\sim 2$ ns FWHM) pulse to excite the NV center efficiently into the $\left|E_{\mathrm{x}}\right\rangle$ state instead of applying a a long (60 ns) pulse to observe multiple Rabi oscillations. We perform this procedure twice, with the collection optics set to collect the fluorescence from either $\left|E_{\mathrm{x}}\right\rangle$ or $\left|\mathrm{E}_{\mathrm{y}}\right\rangle$. We also repeat this procedure in both configurations with the green reionization pulse disabled in order to measure the background due to pulse reflections, ambient light, and APD dark counts. We reject photons collected before $3.3 \mathrm{~ns}$ after the end of the excitation pulse to further remove effects due to pulse reflections, as shown in the inset to Fig. A.3. 


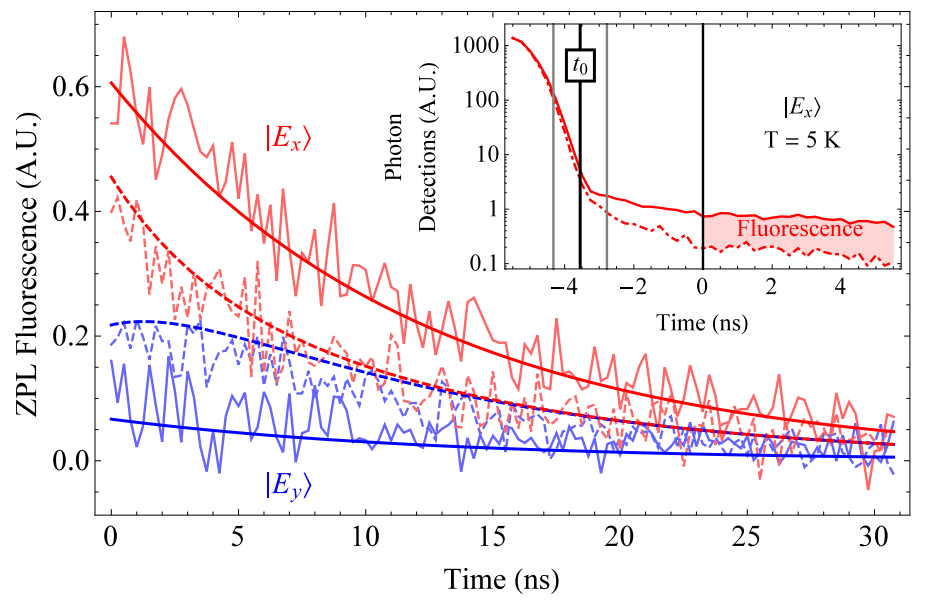

Figure A.3: Background-subtracted fluorescence of $x$ (red) or $y$ (blue) polarization collected after resonant excitation to $\left|\mathrm{E}_{\mathrm{x}}\right\rangle$. The data, which are also shown in Fig. 2(b) in the main text, were taken at $T=5.0 \mathrm{~K}$ (dashed lines) and $T=20 \mathrm{~K}$ (solid lines). The fits are simulations to the three-level system described below. The inset, which shares a common $t=0$ ns point with the main graph, shows the $x$-polarized photons collected with (solid) and without (dashed) the green reionization pulse. We reject all photons collected in the first $3.3 \mathrm{~ns}$ after the end of the reflected excitation pulse, as indicated by the shaded region beginning at $t=0 \mathrm{~ns}$. We indicate the starting point $t_{0}$ of the mixing/radiative decay dynamics (with $95 \%$ confidence bounds), as predicted by fitting the fluorescence data to the three-level model described in the text.

\section{A.4 Fluorescence depolarization analysis}

We would like to test whether the Rabi decoherence and fluorescence polarization measurements give a consistent picture of population mixing. We will take the values of $\Gamma_{\text {Mix }}$ that we extract from the Rabi decoherence data and use them to simulate the fluorescence depolarization. For the sake of simplicity, we restrict our analysis to after the excitation pulse and we ignore mixing dynamics during the pulse. Therefore, we assume that the NV has efficiently been excited into $\left|\mathrm{E}_{\mathrm{x}}\right\rangle$ and there is initially no population in $\left|\mathrm{E}_{\mathrm{y}}\right\rangle$. 
We consider a three-level system: the bright state $|B\rangle$ corresponds to $\left|\mathrm{E}_{\mathrm{x}}\right\rangle$, the dark state $|D\rangle$ corresponds to $\left|\mathrm{E}_{\mathrm{y}}\right\rangle$, and the ground state $|G\rangle$ corresponds to $|0\rangle$. We solve the population rate equations

$$
\begin{aligned}
& \dot{\rho}_{B}=-\Gamma_{\text {Rad }} \rho_{B}-\Gamma_{\text {Mix }}\left(\rho_{B}-\rho_{D}\right) \\
& \dot{\rho}_{D}=-\Gamma_{\text {Rad }} \rho_{D}+\Gamma_{\text {Mix }}\left(\rho_{B}-\rho_{D}\right),
\end{aligned}
$$

where $\Gamma_{\text {Rad }}$ is the radiative decay rate from both $|B\rangle$ and $|D\rangle$ to the ground state and $\Gamma_{\text {Mix }}$ is the mixing rate between $|B\rangle$ and $|D\rangle$, with the initial conditions $\rho_{B}(0)=$ $1, \rho_{D}(0)=0$ to find

$$
\begin{aligned}
\rho_{B}(t) & =\frac{1}{2} e^{-\Gamma_{\mathrm{Rad}} t}\left(1+e^{-2 \Gamma_{\mathrm{Mix}} t}\right) \\
\rho_{D}(t) & =\frac{1}{2} e^{-\Gamma_{\mathrm{Rad}} t}\left(1-e^{-2 \Gamma_{\mathrm{Mix}} t}\right) .
\end{aligned}
$$

To fit the observed fluorescence, we need to account for the imperfect polarization selectivity. We fit the fluorescence data shown in Fig. A.3 to

$$
\begin{aligned}
& \tilde{\rho}_{B}(t)=A\left[(1-\epsilon) \rho_{B}\left(t-t_{0}\right)+\epsilon \rho_{D}\left(t-t_{0}\right)\right] \\
& \tilde{\rho}_{D}(t)=A\left[(1-\epsilon) \rho_{D}\left(t-t_{0}\right)+\epsilon \rho_{B}\left(t-t_{0}\right)\right],
\end{aligned}
$$

where $\epsilon$ is the error in our polarization selectivity. We extract the mixing rates $\Gamma_{\text {Rad }}(5.0 \mathrm{~K})=2 \pi \times 0.08 \mathrm{MHz}$ and $\Gamma_{\mathrm{Rad}}(20 \mathrm{~K})=2 \pi \times 18.5 \mathrm{MHz}$ from the fit to the Rabi decoherence data described in Section A.1. We fit all four data sets simultaneously, using these two values of $\Gamma_{\text {Rad }}$ and a common set of fit parameters, finding

$$
\begin{aligned}
A & =0.90 \pm 0.06 \\
t_{0} & =-3.6 \pm 0.8 \mathrm{~ns} \\
\epsilon & =10 \pm 2 \% .
\end{aligned}
$$


We find excellent agreement between the simulation fit and our data. The polarization selectivity of $1-\epsilon=90 \%$ is roughly consistent with our expectations of the collection path's performance. The nonnegligible value of $\epsilon$ reflects the necessary tradeoffs inherent our choice of polarization settings, as discussed in the previous section.

The value of $t_{0}$ is also consistent with our expectations. Our simplified model of an undriven three-level system subject only to radiative decay and nonradiative population transfer is necessarily valid only after the end of the excitation pulse. The value of $t_{0}$ extracted from the fit places $t_{0}$ near the end of the excitation pulse's falling edge, as shown in the inset to Fig. A.3. Essentially, the simulation, when extrapolated backward toward the excitation pulse, picks out the time that marks the beginning of the underlying model's validity. A more precise statement would require a model that incorporates the resonant driving dynamics that occur during the excitation pulse as well as the nonnegligible ( $\sim 2 \mathrm{~ns}$ ) width of the pulse's falling edge, which is beyond the scope of this analysis. The success of the simplified model, however, provides strong evidence that the Rabi decoherence and fluorescence polarization measurements give a consistent picture of population mixing.

\section{A.5 Excited state lifetime measurement}

To measure the lifetimes of $\left|A_{1}\right\rangle,\left|A_{2}\right\rangle$, and $\left|E_{1,2}\right\rangle$, we again employ an experimental procedure similar to that depicted in Fig. A.1. In this case, however, the primary excitation laser, labeled " $E_{x}$ " in the figure, is tuned to the transition between \pm 1 and one of the ${ }^{3} E$ states listed. We apply a short excitation pulse, as in the fluorescence 
depolarization measurement. We repump on the $|0\rangle \rightarrow\left|\mathrm{E}_{\mathrm{y}}\right\rangle$ transition for $10 \mu$ s to repopulate the $| \pm 1\rangle$ states; we repump for a longer time because the $\left|E_{y}\right\rangle$ transition is more closed than the $\left|A_{1}\right\rangle$ transition previously used to repump to the correct spin state. We perform this procedure at several temperatures between $5 \mathrm{~K}$ and $26 \mathrm{~K}$.

To extract the excited state lifetimes, we fit each dataset to a single-exponential decay function. The fit window starts $4 \mathrm{~ns}$ after the beginning of the excitation pulse and extends for $115 \mathrm{~ns}$. This $4 \mathrm{~ns}$ delay was selected to remove any effects of the excitation pulse, ensuring that the fluorescence we consider is solely the result of spontaneous emission.

\section{A.6 Intersystem crossing rate from $\left|E_{x}\right\rangle$}

We now justify the assumption that the ISC rate from $\left|E_{\mathrm{x}}\right\rangle$ is negligible, which enables the state-dependent ISC rates shown in Fig. 3 in the main text to be extracted from the measured fluorescence lifetimes. The ratio of the ISC rate from the ${ }^{3} E$ states with $\left|m_{s}\right|=1\left(\Gamma_{\mathrm{ISC}, \pm 1}\right)$ to the ISC rate from the ${ }^{3} E$ states with $m_{s}=0\left(\Gamma_{\mathrm{ISC}, 0}\right)$ has been addressed both theoretically and experimentally [97, 44, 99].

Manson et al. [97] developed a detailed model of NV center spin dynamics from a careful consideration of the NV center's symmetry properties. Their model predicts $\Gamma_{\text {ISC }, 0}=0$, a conclusion that is consistent both with their measurements of the NV center's transient behavior under nonresonant optical excitation and with the conclusions of an earlier review of nonresonant spin initialization and readout [44], which cited $\Gamma_{\mathrm{ISC}, 0} \sim 10^{3} \mathrm{~s}^{-1}$ and $\Gamma_{\mathrm{ISC}, \pm 1} \sim 10^{6} \mathrm{~s}^{-1}$. This conclusion is somewhat inconsistent, however, with the more recent observations of Robledo et al. [99], who used 
nonresonant excitation to measure the spin-dependent lifetimes of the ${ }^{3} E$ states, the lifetime of the metastable $\left|{ }^{1} E_{1,2}\right\rangle$ states, and the degree of spin polarization in the ${ }^{3} E$ manifold. They used these measurements to construct a phenomenological model of NV center dynamics, from which they extracted $\Gamma_{\text {ISC }, 0} / 2 \pi \approx 1-2 \mathrm{MHz}$. Similarly, Tetienne et al. [231] applied measurements of photoluminescence intensity, ESR contrast, and ${ }^{3} E$ state lifetimes as functions of an off-axis magnetic field to the same model to extract $\Gamma_{\mathrm{ISC}, 0} / 2 \pi \approx 0.8-1.7 \mathrm{MHz}$.

The question of $\Gamma_{\mathrm{ISC}, E_{x}}$ is, in general, complicated by the facts that there is significant phonon-induced depolarization between $\left|\mathrm{E}_{\mathrm{x}}\right\rangle$ and $\left|\mathrm{E}_{\mathrm{y}}\right\rangle$ for $T>15 \mathrm{~K}$ and that the $\left|\mathrm{E}_{\mathrm{y}}\right\rangle$ and $\left|E_{1,2}\right\rangle$ states exhibit a level anticrossing when the strain-induced $\left|\mathrm{E}_{\mathrm{x}}\right\rangle-\left|\mathrm{E}_{\mathrm{y}}\right\rangle$ splitting is approximately $7 \mathrm{GHz}[232]$. Thus, phonons couple $\left|\mathrm{E}_{\mathrm{x}}\right\rangle$ to $\left|\mathrm{E}_{\mathrm{y}}\right\rangle$, spin-spin interaction couples $\left|\mathrm{E}_{\mathrm{y}}\right\rangle$ to $\left|E_{1,2}\right\rangle$, and $\left|E_{1,2}\right\rangle$ decay to $\left|{ }^{1} A_{1}\right\rangle$ through the ISC mechanism described in Ref. [104]. The $\Gamma_{\mathrm{ISC}, E_{x}}$ due to such a mechanism would depend on both temperature and crystal strain. Because both Robledo and Tetienne considered the NV center at $T=300 \mathrm{~K}$ in their models and neither specified the $\left|E_{x}\right\rangle-\left|E_{y}\right\rangle$ splitting, this mechanism could be responsible for their non-negligible values of $\Gamma_{\mathrm{ISC}, 0}$. In the context of this work, however, the fact that the measured lifetime of $\left|E_{\mathrm{x}}\right\rangle$ does not depend on temperature from $5 \mathrm{~K}$, where mixing between $\left|E_{\mathrm{x}}\right\rangle$ and $\left|\mathrm{E}_{\mathrm{y}}\right\rangle$ is negligible, to $26 \mathrm{~K}$, where mixing is much faster than radiative decay, indicates that the contribution of this mechanism is negligible.

We can also place a limit of $\Gamma_{\mathrm{ISC}, E_{x}}$ based on the measured coherence time of optical Rabi oscillations. If we consider the low-temperature limit, where mixing between $\left|E_{x}\right\rangle$ and $\left|E_{y}\right\rangle$ is suppressed, then we can perform an analysis analogous to 


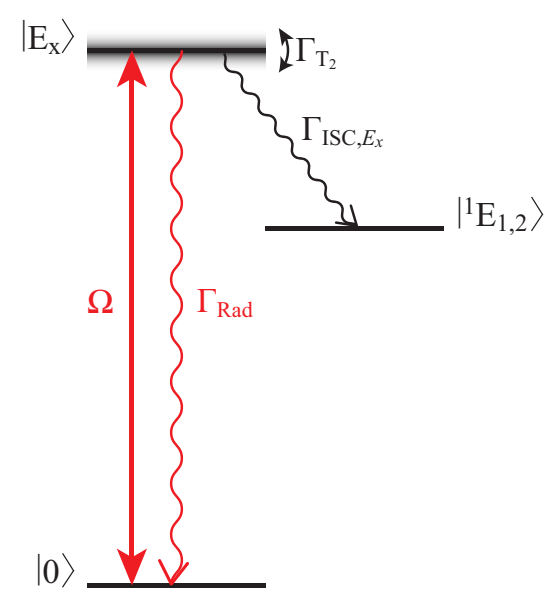

Figure A.4: The three-level system used to extract $\Gamma_{\mathrm{ISC}, E_{x}}$ from a measurement of optical Rabi oscillations conducted at $5.8 \mathrm{~K}$. Because the oscillation and ISC dynamics that occur over a timescale of $\sim 4$ to $60 \mathrm{~ns}$ and the lifetimes of the singlet states are $\tau_{1} A_{1}=0.9 \mathrm{~ns}$ [111] and $\tau_{E_{1,2}} \sim 370 \mathrm{~ns}$ [99], we assume that population neither returns from $\left|{ }^{1} A_{1}\right\rangle$ to $\left|\mathrm{E}_{\mathrm{x}}\right\rangle$ nor decays from $\left|{ }^{1} E_{1,2}\right\rangle$ to $|0\rangle$ once it has undergone the ISC transition to the singlet states. Thus, we can combine $\left|{ }^{1} A_{1}\right\rangle$ and $\left|{ }^{1} E_{1,2}\right\rangle$ into one effective dark state.

that described in Sec. A.2, where we substitute the ISC crossing to the singlet states for phonon-induced mixing to $\left|\mathrm{E}_{\mathrm{y}}\right\rangle$. The resulting three-level system is shown in Fig. A.4. We solve the corresponding master equation in Lindblad form and find that the emitted fluorescence $\left(\propto \rho_{E_{x} E_{x}}\right)$ oscillates within an envelope given by

$$
g(t)=\frac{1}{2}\left(e^{-t / \tau_{\mathrm{Rabi}}}+1\right) e^{-\Gamma_{\mathrm{ISC}, E_{x}} t / 2},
$$

where the exponential timescale is

$$
\frac{1}{\tau_{\text {Rabi }}}=\frac{3}{4} \Gamma_{\text {Rad }}^{(x)}+\frac{1}{2} \Gamma_{T_{2}} .
$$

We fit the fluorescence during the Rabi oscillations to

$$
f(t)=A\left[\cos (\Omega t-\phi) e^{-\left(t-t_{0}\right) / \tau_{\mathrm{Rabi}}}+1\right] e^{-\Gamma_{\mathrm{ISC}, E_{x}} t / 2},
$$


where all quantities except for $t$ are free fit parameters. The extracted initial time $t_{0}$ of the Rabi oscillation decay is found to be close (within $3 \mathrm{~ns} \sim \tau_{\pi}$ ) to the start of the excitation pulse in all cases, indicating that the visibility of the Rabi oscillations is well described by Eq. A.16.

From these fits, we extract $\Gamma_{\mathrm{ISC}, E_{x}} / 2 \pi=0.62 \pm 0.21 \mathrm{MHz}$. We note that our model does not take into account spin non-preserving radiative decay from $\left|E_{\mathrm{x}}\right\rangle$ into the dark $| \pm 1\rangle{ }^{3} A_{2}$ states or deionization into the dark $\mathrm{NV}^{0}$ charge state, both of which would mimic the effect of the ISC transition into the dark metastable singlet state. Thus, this value represents an upper bound on $\Gamma_{\mathrm{ISC}, E_{x}}$ that is an order of magnitude lower than the measured $\Gamma_{\mathrm{ISC}, \pm 1}$. We can therefore assume that $\Gamma_{\mathrm{ISC}, E_{x}}$ is negligible, in agreement with the preponderance of literature cited above.

\section{A.7 Intersystem crossing rate analysis}

In Fig. 3.3, we fit the ISC rates observed after excitation into $\left|A_{1}\right\rangle$ or $\left|A_{2}\right\rangle$ to a simple model of phonon-induced state mixing. In this model, we assume the following sequence of events. At $t=-t_{0}$, we instantaneously transfer the entire population to the target state with a perfect $\pi$ pulse. Phonons induce state mixing at a rate $\Gamma_{\text {Mix }}$ until $t=0$, at which point we begin fitting the fluorescence decay. Mixing continues at a rate $\Gamma_{\text {Mix }}$ throughout the entire fitting period, from $t=0$ to $t=\Delta t$. We then fit the PSB fluorescence observed during the measurement period to a simple exponential. Values of $t_{0}=4 \mathrm{~ns}$ and $\Delta t=115 \mathrm{~ns}$ were chosen to match the analysis performed on the experimental data, as described in the previous section.

To model the dynamics of the system under mixing between $\left|A_{1}\right\rangle$ and $\left|A_{2}\right\rangle$, radia- 
tive decay from both states, and ISC decay from $\left|A_{1}\right\rangle$ alone, we solve

$$
\begin{aligned}
& \dot{\rho}_{A_{1}}=-\left(\Gamma_{\text {Rad }}+\Gamma_{\text {ISC }}\right) \rho_{A_{1}}-\Gamma_{\text {Mix }}\left(\rho_{A_{1}}-\rho_{A_{2}}\right) \\
& \dot{\rho}_{A_{2}}=-\Gamma_{\text {Rad }} \rho_{A_{2}}+\Gamma_{\text {Mix }}\left(\rho_{A_{1}}-\rho_{A_{2}}\right) .
\end{aligned}
$$

The quantity of interest is the measured fluorescence intensity, which is proportional to $\rho_{A_{1}}+\rho_{A_{2}}$. The fluorescence intensity measured after excitation into $A_{1,2}$ is given by

$$
I_{A_{1,2}}=e^{-\left(\Gamma_{\mathrm{Rad}}+\Gamma_{\mathrm{Mix}}+\Gamma_{\mathrm{ISC}} / 2\right) t}\left[\frac{2 \Gamma_{\mathrm{Mix}} \mp \Gamma_{\mathrm{ISC}}}{\Gamma^{\prime}} \sinh \left(\frac{\Gamma^{\prime}}{2} t\right)+\cosh \left(\frac{\Gamma^{\prime}}{2} t\right)\right],
$$

where $\Gamma^{\prime}=\sqrt{\Gamma_{\text {ISC }}^{2}+4 \Gamma_{\text {Mix }}^{2}}$.

We simulate the fluorescence intensity observed during the measurement period using Eq. A.20. We expect that the mixing rate between $\left|A_{1}\right\rangle$ and $\left|A_{2}\right\rangle$ should be equal to that between $\left|E_{x}\right\rangle$ and $\left|E_{y}\right\rangle$ [104]. We therefore set the temperaturedependent $\Gamma_{\text {Mix }}$ equal to the value given by our fit to the $\left|E_{x}\right\rangle-\left|E_{y}\right\rangle$ mixing data, which is described in Sec. A.1.

We then apply the same analysis that we used to extract $\Gamma_{\text {ISC }}$ from our measured fluorescence intensity data, giving the effective ISC rates measured after excitation into $\left|A_{1}\right\rangle$ or $\left|A_{2}\right\rangle$ as functions of temperature and $\Gamma_{A_{1}}$. We perform a $\chi^{2}$ minimization fit to the measured temperature-dependent ISC rates to find $\Gamma_{A_{1}} / 2 \pi=16.0 \pm 0.6$ MHz. 


\section{Appendix B}

\section{Supporting material for Chapter 4}

\section{B.1 Sample information}

The SiV sites were found by looking through a (001) face on a type IIa diamond. The diamond had a high-purity homoepitaxial chemical-vapor-deposition (CVD) layer grown on a low-strain, high pressure high temperature (HPHT) (001)oriented substrate. This layer was grown using a microwave plasma-assisted chemicalvapor-deposition (MPCVD) apparatus assuring a low level of unintentional impurities [233]. The $\mathrm{SiV}$ sites were incorporated in the growth process by etching a piece of silicon-carbide $(\mathrm{SiC})$ with the growth plasma. This technique produced highly uniform centers with a small inhomogeneous distribution[127]. 


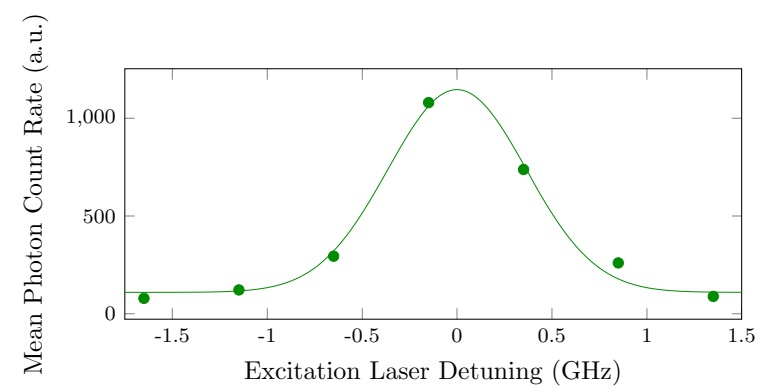

Figure B.1: Inhomogeneous distribution of the $\mathrm{SiV}$ resonance. A Gaussian fit to the distribution reveals a distribution with a width of $\sigma=364.5 \pm 33.0 \mathrm{MHz}$. The shot noise error was estimated to be smaller than the data points.

\section{B.2 Inhomogeneous distribution}

One of the key requirements for a Hong-Ou-Mandel (HOM) experiment using separate emitters in solids is to isolate emitters with identical emission frequencies. For emitters in solids, the inhomogeneous distribution is typically orders of magnitude larger than the optical transition linewidths, and large inhomogeneous distributions require some combination of postselection of emitters or active tuning[ $[75,77,76$, 64]. Owing to the insensitivity of its transitions to electric fields, SiV has a much narrower inhomogeneous distribution as discussed in the main text. Fig. B.1 shows the measured inhomogeneous distribution using the technique described in Fig. 4.3. The total fluorescence counts in a field of view of $\sim 150 \mu \mathrm{m}^{2}$ are plotted for each laser excitation frequency $\nu$. The measured inhomogeneous distribution width of $364.5 \pm$ $33.0 \mathrm{MHz}$ is only a few times the lifetime limited linewidth of $94 \mathrm{MHz}$, suggesting that most of the emitters in the field of view could be used for a HOM demonstration.

Since the SiV centers couple to strain and phonons but not to electric fields, they could be used as a nanoscale sensor of the strain distribution in a crystal. For 


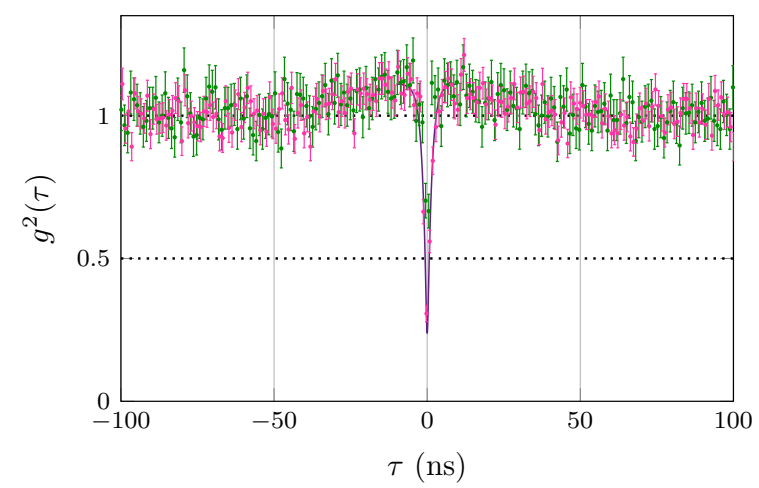

Figure B.2: Normalization for the HOM results. The long time dynamics of the second order intensity correlation function $g^{2}(\tau)$ are shown where the data was coarse binned with 1024 ps intervals. The $g^{2}(\tau)$ function was normalized using the steady state coincidence levels at long time delays where effects of bunching[234] for $|\tau|>50$ ns vanish.

example, the bright emitter in Fig. 4.3(b) was on the wings of the inhomogeneous distribution, possibly due to a point defect near the emitter that shifts its frequency substantially with respect to the rest of the ensemble.

\section{B.3 Time dynamics of $g^{2}(\tau)$}

Here we describe the solid curves in Fig. 4.4 that represents our model. The shape of the measured second-order correlation function, $g^{2}(\tau)$, for short time delays can be explained using the expression

$$
g^{2}(\tau)=\frac{1}{2} g_{1}^{(2)}(\tau)+\frac{1}{2}\left(1-\chi\left|g_{1}^{(1)}(\tau)\right|^{2} \cos (\Delta \tau)\right)
$$

where $g_{1}^{(1)}$ and $g_{1}^{(2)}$ are the field (first-order) and intensity (second-order) autocorrelation functions for a single emitter. $\Delta$ is the detuning between the two emitters, $\chi=1(0)$ for indistiguishable(distinguishable) photons $[75,64]$. We substitute 
$g_{1}^{(2)}(\tau)=1-\left(1-c_{\mathrm{B}}\right) \exp \left(-\frac{|\tau|}{\tau_{0}}\right)$ and $\left|g_{1}^{(1)}(\tau)\right|^{2}=\left(1-c_{\mathrm{B}}\right) \exp \left(-\frac{|\tau|}{\tau_{c}}\right)$, where $\tau_{0}=1.73$ $\mathrm{ns}$ is the excited state lifetime and $\tau_{c}=1.18 \mathrm{~ns}$ is the coherence time of the transition calculated from the linewidths of the PLE spectra. The only free parameter in this model is the ratio of the background events to total events, $c_{\mathrm{B}}$, which we consistently find to be 0.12 from the fits to control and HOM experiments. For the HOM measurement, the excitation intensity was below saturation and the emission intensity from each emitter was balanced at the detectors. The detected count rates after the etalons was $\sim 6 \mathrm{kcts} / \mathrm{s}$ from each emitter. In our model we also take into account the effects of detector timing jitter which was characterized using a pulsed laser. The timing jitter of our detectors can be modeled as a gaussian with $\sigma \sim 150 \mathrm{ps}$ for each detector (PicoQuant $\tau$-SPAD). To fit the HOM data, we used an analytical expression which is a convolution of the expression above with the detector timing response. The model and the data were normalized such that $g^{2}(|\tau| \rightarrow \infty)=1$ as shown in Figure B.2 to take the weak bunching effect into account. The bunching effect is related to the population dynamics of a single emitter and hints at the presence of a metastable state [234]. 


\section{Appendix C}

\section{Supporting material for Chapter 6}

\section{C.1 Experimental setup}

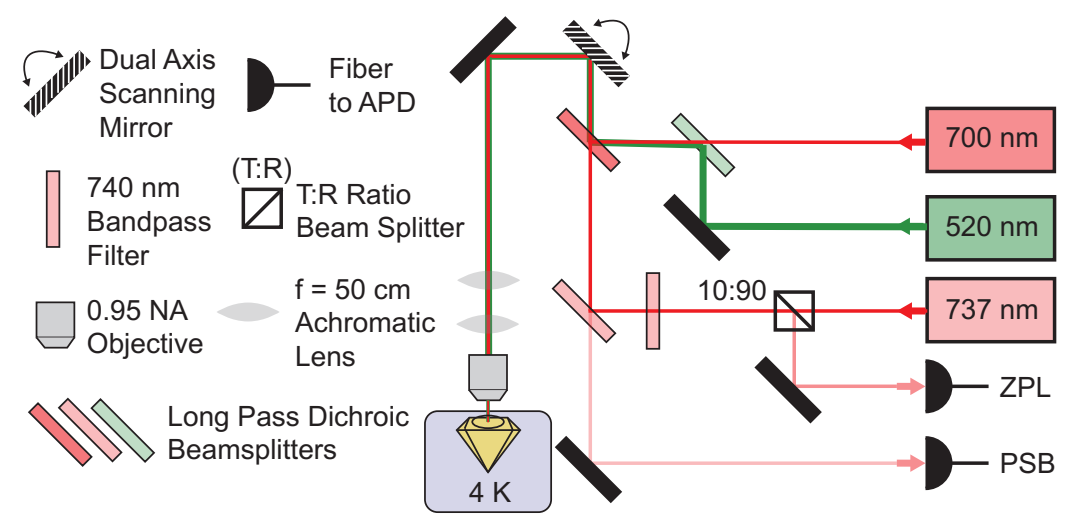

Figure C.1: Confocal microscope design. The $520 \mathrm{~nm}$ and $700 \mathrm{~nm}$ lasers are used to excite the SiV off-resonantly. The $737 \mathrm{~nm}$ external-cavity diode laser is used to excite the $\mathrm{SiV}$ resonantly. Collection can be performed either on the ZPL (if the excitation is off-resonance) or the PSB (in either excitation scheme).

The experiments were carried out using home-built scanning confocal microscopes as illustrated in Fig. C.1. The three lasers used for excitation $(520 \mathrm{~nm}$ and $700 \mathrm{~nm}$ 
diode lasers used for off resonant excitation, $737 \mathrm{~nm}$ external-cavity diode laser used for resonant excitation) are combined using dichroic beamsplitters. A $760 \mathrm{~nm}$ longpass dichroic beamsplitter separates the PSB fluorescence from the rest of the optical channels. An additional bandpass filter $(740 \pm 13 \mathrm{~nm})$ is used on the ZPL channel. Single photons are detected using single photon counting modules (Picoquant $\tau$-SPAD and Excelitas SPCM-NIR). The cryogenic measurements were performed in $4 \mathrm{~K}$ helium flow cryostats. We used a $0.95 \mathrm{NA}$ microscope objective (Nikon CFI LU Plan Apo Epi 100×) in all experiments. During the cryogenic measurements, the objective was inside the vacuum chamber and the sample was clamped with an indium foil spacer to the cold finger of the cryostat. During the PLE measurements, the $520 \mathrm{~nm}$ laser is pulsed at a $\sim 5 \%$ duty cycle to stabilize the charge state of the $\mathrm{SiV}$ center$[49,54]$. The detectors are gated off during these pulses.

\section{C.2 Fluorescence autocorrelation measurements}

To verify our ability to create single $\mathrm{SiV}$ centers, we performed fluorescence autocorrelation measurements on $\mathrm{SiV}$ centers inside diamond nanobeams. We performed this measurement by exciting the $\mathrm{SiV}$ centers off resonantly as described above and splitting the emission between two detectors in a Hanbury-Brown-Twiss configuration. The relative arrival times of the photons on the two detectors were recorded using fast acquisition electronics (PicoQuant HydraHarp 400) with a resolution better than $128 \mathrm{ps}$. In this experiment, our total average photon count rate from this $\mathrm{SiV}$ was $9 \times 10^{4}$ counts per second.

The relative photon detection times $g^{(2)}(\tau)$ (normalized by defining $g^{(2)}(\infty)=1$ ) 


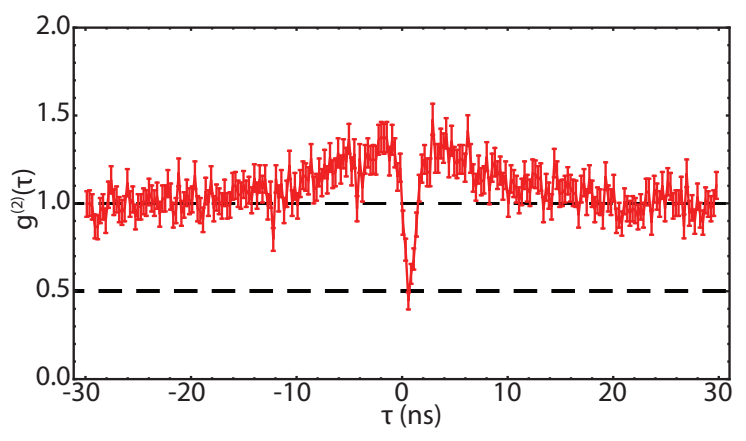

Figure C.2: Fluorescence autocorrelation measurement of a $\mathrm{SiV}$ center inside a diamond nanobeam as described in the text. Error bars are estimated assuming the noise on the number of detected photons follows a Poisson distribution (shot noise). The extent of the dip at $\tau=0$ is limited by finite detector bandwidth: we measure $g^{(2)}(0)=0.45$; deconvolving the detector response yields $g^{(2)}(0)=0.15$.

from a representative SiV are displayed in Fig. C.2. A value of $g^{(2)}(0)<0.5$ would confirm that we are measuring emitters producing single photons. Finite jitter on our detectors of around $350 \mathrm{ps}$ causes the measured arrival times of our photons to be convolved with the detector response, hence limiting the sharpness and minimum value of our dip. Fitting the data (including the detector response) using previously reported methods[142] gives a value $g^{(2)}(0)=0.45$. Deconvolving the detector response gives a value $g^{(2)}(0)=0.15$, indicating that the extent of our $g^{(2)}(0)$ dip is limited primarily by detector response as expected. 


\section{Appendix D}

\section{Supporting material for Chapter 7}

\section{D.1 Setup description}

The experiments in Chapter 7 are carried out in a confocal microscope consisting of a modified $4 \mathrm{~K}$ liquid helium flow probe-station (Desert Cryogenics model TTP4) and a 0.95 NA microscope objective (Nikon CFI LU Plan Apo Epi 100×) inside the vacuum chamber. The layout of the setup is shown in Fig. D.1. Three ports are used to excite the system (Ch. 1), control the $\mathrm{SiV}$ and detect fluorescence (Ch. 2) and measure transmission (Ch. 3).

For the coarse characterization of the diamond nanocavity resonances, we excite the system with a broadband supercontinuum laser (NKT Photonics SuperKExtreme). The transmitted light is sent to a spectrometer (Horiba iHR550 with Synapse CCD and $1800 \mathrm{gr} / \mathrm{mm}$ ) with a spectral resolution of $0.025 \mathrm{~nm}$. To obtain high resolution spectra of the system in Fig. 7.1, we scanned a Ti:Sapphire laser (Probe laser: M-Squared SolsTiS-2000-PSX-XF) across the cavity and atomic resonance. For all 
frequency scans, the instantaneous laser frequency was monitored using a high resolution wavemeter (High Finesse WS7) with $10 \mathrm{MHz}$ resolution and $50 \mathrm{MHz}$ accuracy. We used a laser noise eater (Thorlabs LCC3112H) to stabilize laser power to less than $1 \%$ during frequency scans.

We use a home-built external-cavity diode laser (Opnext Diode HL7302MG, Littrow configuration) with an electro-optical modulator (EOM: EOSPACE interferometric electro-optical amplitude modulator, model AZ-AV5-40-PFA-PFA-737 with $40 \mathrm{GHz}$ bandwidth) to apply short (minimum pulse duration 500 ps) optical pulses for the lifetime measurements and switching experiment (Figs. 7.1 and 7.2). The interferometer of the EOM was stabilized using a lock-in amplifier (SRS model SR830) and short driving electrical pulses were generated using an arbitrary waveform generator (Tektronix AWG710, $4 \mathrm{GSa} / \mathrm{s}$ ). Off-resonant excitation of the $\mathrm{SiV}$ was performed with $700 \mathrm{~nm}$ (Thorlabs LP705-SF15) and $520 \mathrm{~nm}$ (Thorlabs LP520-SF15) diode lasers. Single photons were counted using single-photon detectors $(2 \times$ PicoQuant $\tau$-SPAD, and $2 \times$ Excelitas SPCM-NIR) and time tagged using fast acquisition electronics (PicoQuant HydraHarp 400).

The waveguides described in Sec. D.2 support two modes with different polarizations. In our design, the cavity mode is a transverse-electric-like (TE) mode. At the cavity resonance frequency, there is a high-transmission passband for transversemagnetic-like (TM) modes. (Our conventions for TE and TM in this context are described in Section D.2.1.) In order to only excite the relevant (TE) cavity mode, we place a polarizer immediately before the objective.

The in- and out-coupling efficiencies between free-space and waveguide modes are 


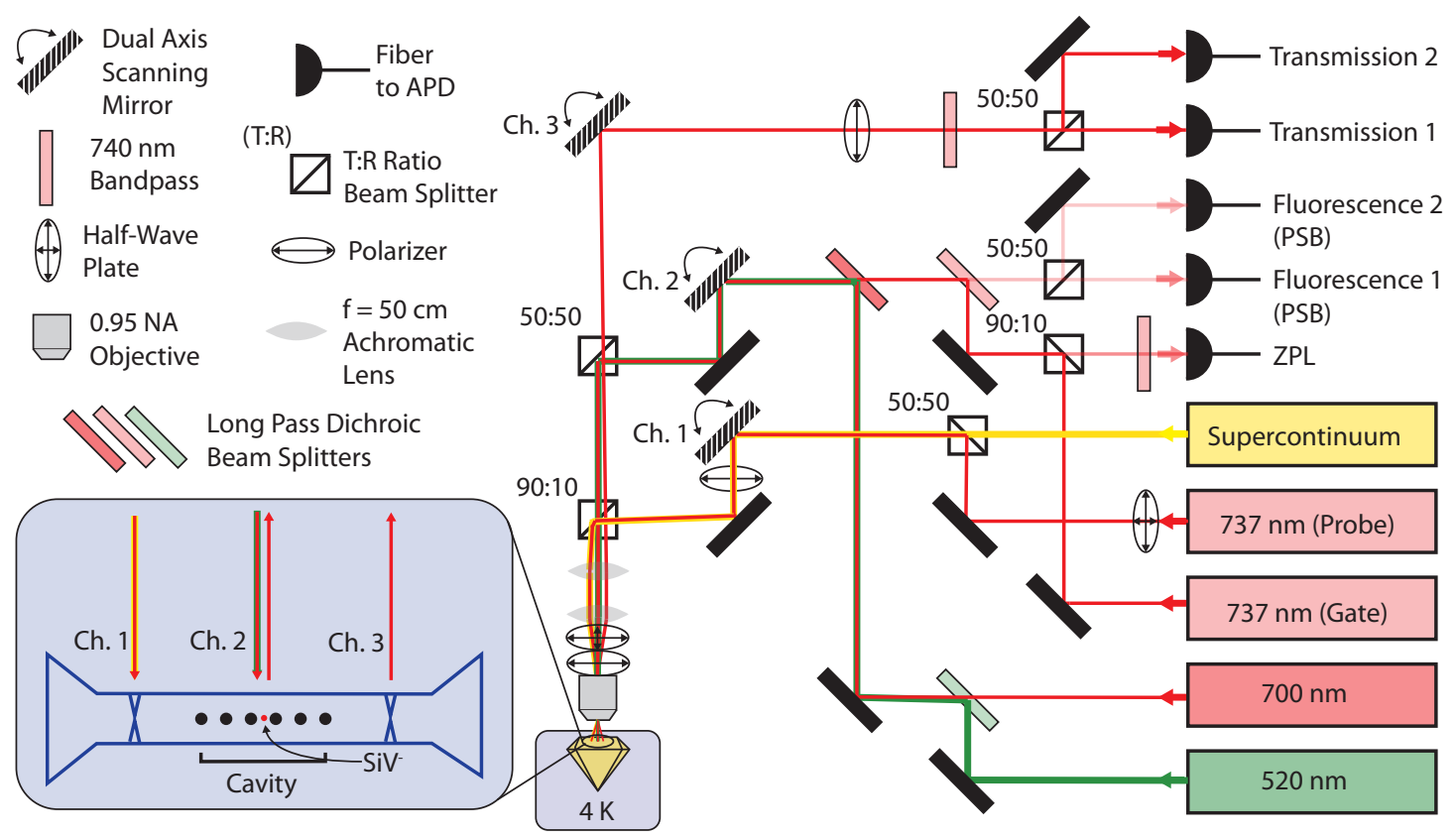

Figure D.1: Optical configuration for the three-port measurements described in Chapter 7. Each port has its own dual-axis scanning galvo mirror system (Thorlabs GVS012) labeled Ch. 1-Ch. 3. The first port, Ch. 1, is used to excite the waveguide mode and contains both a supercontinuum light source and a narrowband Ti:Saph laser used for broadband and narrowband characterization of the system response respectively. The second port, Ch. 2, is used to control the $\mathrm{SiV}$ (as described in Sec. D.4) and monitor the $\mathrm{SiV}$ fluorescence scattering in the phonon-sideband (PSB). The third port, Ch. 3, is used to measure the transmission through the cavity mode. The objective and the sample are in vacuum, and the sample is cooled to $4 \mathrm{~K}$. Shaded blue inset: schematic of the spatial position of the focal spots of the three channels relative to the SiV-cavity system. 
each of order $1 \%$ based on simulations. In order to isolate transmission from the diamond nanocavity and mitigate residual reflection from free-space optics, we use a cross-polarization scheme between the excitation (Ch. 1) and transmission (Ch. 3) channels.

\section{D.2 Device design, fabrication and characteriza- tion}

\section{D.2.1 Cavity design}

The one-dimensional diamond photonic-crystal cavity ("nanobeam cavity" hereafter) used here (based on previous designs[195]) consists of a diamond waveguide with a triangular cross-section perforated by a chirped 1D lattice of elliptically-shaped air holes. The unit cell of the lattice (Fig. D.2A) is parameterized by the etch angle $(\theta)$, width $(w)$, lattice constant $(a)$ and major (transverse) and minor (longitudinal) elliptical air hole diameters $\left(d_{z}, d_{x}\right)$. The unit cell etch angle (the half-angle at the bottom apex of the triangular cross-section) was fixed at $\theta=50^{\circ}$ in the design to ease fabrication.

Fig. D.2B shows the photonic band structure for a nominal unit cell with $\theta=$ $50^{\circ}$ and $\left(a, w, d_{z}, d_{x}\right)=(260,470,140,140) \mathrm{nm}$. Here, transverse-electric-like (TE, solid black lines) and transverse-magnetic-like (TM, dashed blue lines) guided modes give rise to symmetry based quasi-bandgaps sufficient to realize highly localized resonances. In our convention, the TE modes have odd vector symmetry with respect to reflection across the $z=0$ longitudinal symmetry plane of the nanobeam (see Fig. D.2 


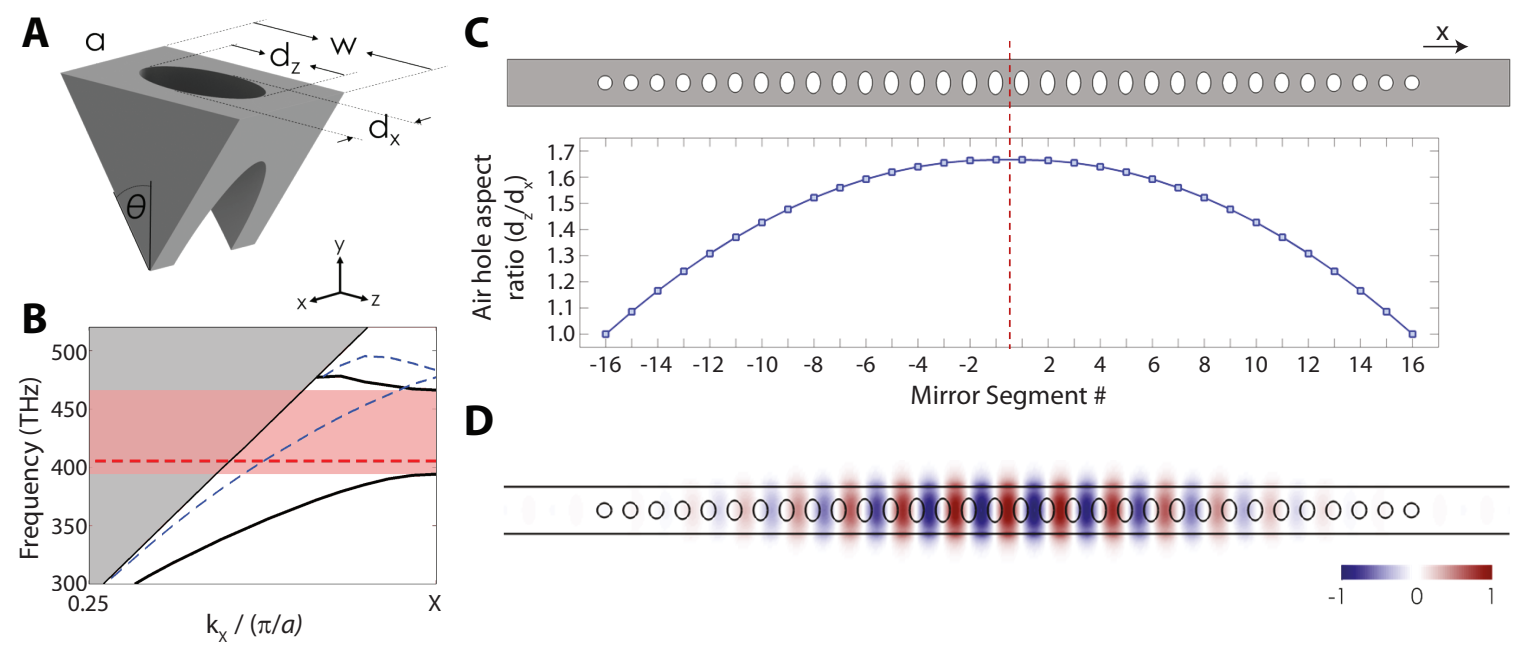

Figure D.2: (A)Schematic of a triangular cross-section diamond unit cell parameterized by the etch angle $(\theta)$, width $(w)$, lattice constant $(a)$, and major and minor elliptical air hole diameters $\left(d_{z}, d_{x}\right)$. (B) Corresponding photonic bandstructure of a nominal unit cell with $\theta=50^{\circ}$ and $\left(a, w, d_{z}, d_{x}\right)=(260,470,140,140) \mathrm{nm}$. The gray shaded region indicates the continuum of radiation and leaky modes that exist above the light line. Below the light line, supported transverse-electric-like (TE) and transverse-magnetic-like (TM) guided modes are indicated by solid black and dashed blue lines, respectively. A quasi-bandgap based on symmetry for the TE guided modes is indicated by the pink shaded region. The fundamental cavity resonance at $\lambda_{T E}=$ $743 \mathrm{~nm}$ is designated by the dashed red line. (C) Schematic of the 16-hole-array cavity design with the air hole aspect ratio $\left(d_{z} / d_{x}\right)$ plotted as a function of mirror segment number. (D) Normalized optical $\mathrm{E}_{\mathrm{y}}$ field profile of the fundamental localized cavity mode of diamond nanobeam cavity design in (C). 
for coordinate conventions). In other words, the electric field of a TE (TM) mode is mostly perpendicular (parallel) to the $z=0$ plane. At the center of the beam, the electric field vector of the TE modes matches the transition dipole moment of two (out of four possible) orientations of the $\mathrm{SiV}$ center up to a factor of $\cos \left(35^{\circ}\right)$.

We parameterized the nanobeam cavity design by the target fundamental TE cavity mode resonance wavelength in free space, $\lambda_{T E}$. Our final design has the following parameters: a nanobeam width $w=0.635 \lambda_{T E}$, lattice constant (hole spacing) $a=0.349 \lambda_{T E}$ and elliptical hole minor diameter $d_{x}=0.191 \lambda_{T E}$, with the major diameter of the elliptical hole increased quadratically, from $d_{z}=d_{x}=0.191 \lambda_{T E}$ at the end of the cavity, to $d_{z}=0.317 \lambda_{T E}$ at the center. This quadratically tapering major diameter is schematically displayed in Fig. D.2C for a 16 hole array on each side of the cavity $\mathrm{x}$-axis mirror plane. For the unit cell dimensions used to calculate the bandstructure in Fig. D.2A, the fundamental TE cavity resonance is located at $\lambda_{T E}=743 \mathrm{~nm}$, which is designated by the dashed red line in Fig. D.2B.

The total cavity loss is comprised of both radiation losses into free-space $\left(Q_{r a d}\right)$ and coupling losses to the feeding waveguide $\left(Q_{w g}\right)$. For the cavity design used in the experiment, the cavity figures of merit generated from FDTD simulations are the cavity mode volume $\left(V=1.8\left(\lambda_{T E} / n\right)^{3}\right.$ with $\left.n=2.4\right)$ and the partial optical Q-factors $\left(Q_{\text {rad }} \approx 320000, Q_{w g} \approx 10000\right)$. From the partial optical Q-factors, the total cavity loss $\left(Q_{t o t}\right)$ is given by the relation $Q_{t o t}=\left(Q_{\text {rad }}^{-1}+Q_{w g}^{-1}\right)^{-1} \approx 10000$. Additionally, the predicted on-resonance transmission of the fundamental cavity mode was calculated by the relation: $T=Q_{t o t}^{2} / Q_{w g}^{2} \approx 94 \%$. It is important for this transmission to be high because it is directly proportional the number of photons we are able to measure 
experimentally.

In order to estimate the transmission of the cavities used in the experiment, the cavity transmission was normalized to transmission through a diamond waveguide (no holes) with identical in- and out-coupling structures. Using this approach, we found that the cavity transmission on resonance was $\geq 85 \%$ of the waveguide transmission.

\section{D.2.2 Cavity fabrication}

Electronic grade, $\langle 100\rangle$-normal oriented, single-crystal diamond substrates (CVD grown, $<5 \mathrm{ppb}[\mathrm{N}]$, Element Six), were first polished to a surface roughness $<5 \mathrm{~nm}$ RMS, followed by an acid treatment ("tri-acid clean" hereafter) in a boiling mixture consisting of equal parts conc. sulfuric acid, conc. nitric acid, and $70 \%$ (aqueous) perchloric acid. Prior to device fabrication, approximately the top six microns of the diamond surface were removed in a standard inductively-coupled-plasma reactiveion etcher (ICP-RIE, Unaxis Shuttleline). This pre-fabrication etch consisted of an $\mathrm{Ar} / \mathrm{Cl}_{2}$ etch (30 minutes, $400 \mathrm{~W}$ ICP power, $250 \mathrm{~W}$ RF power, $25 \mathrm{sccm}$ Ar flow rate, $40 \mathrm{sccm} \mathrm{Cl}_{2}$ flow rate, 8 mTorr chamber pressure) followed by an $\mathrm{O}_{2}$ etch (30 minutes, $700 \mathrm{~W}$ ICP power, $100 \mathrm{~W}$ RF power, $50 \mathrm{sccm} \mathrm{O}_{2}$ flow rate, $10 \mathrm{mTorr}$ chamber pressure). This pre-fabrication etch removes polishing-induced mechanical strain near the top surface of the diamond and reduces the final surface roughness of the diamond substrate to $<1 \mathrm{~nm}$ RMS (confirmed by AFM).

Our nanofabrication procedure is outlined schematically in Fig. D.3. First, a silica etch mask was patterned on the prepared diamond substrates using electron-beam lithography on a $650 \mathrm{~nm}$ spin-coated layer of $83 \%$ hydrogen silsesquioxane (HSQ, 
A (i)
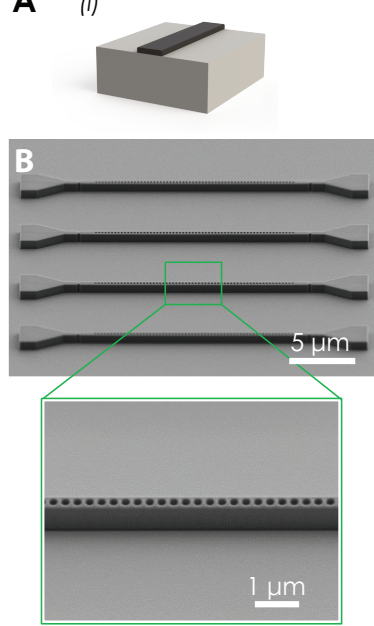

(ii)
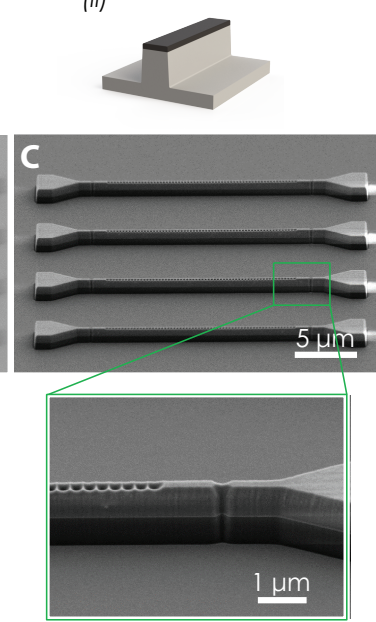

(iii)
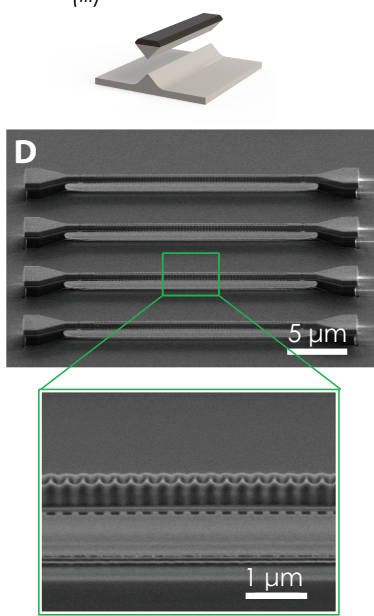

(iv)
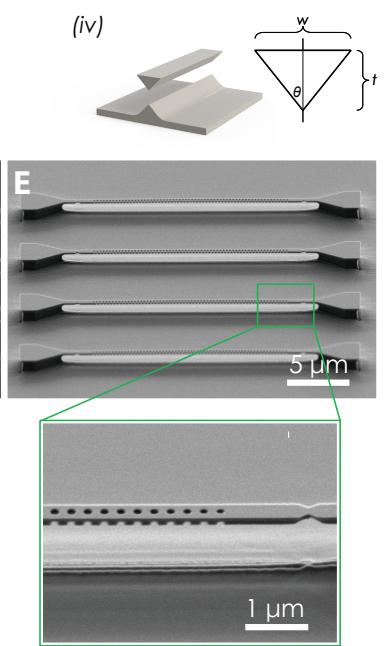

Figure D.3: (A) Illustration of the angled-etching technique used to fabricate freestanding optical nanocavity structures in bulk single-crystal diamond. Angled-etching fabrication steps with corresponding SEM images: (i) define an etch mask on substrate via standard fabrication techniques (panel (B)), (ii) transfer etch mask pattern into the substrate by top-down plasma etching (panel (C)), (iii) employ angled-etching to realize suspended nanobeam structures (panel (D)), (iv) remove residual etch mask (panel (E)). All SEM images taken at a stage tilt of $60^{\circ}$.

FOX-16 from Dow Corning) negative resist in methyl isobutyl ketone (MIBK). Exposed HSQ was developed in tetramethylammonium hydroxide (TMAH, 25\% diluted aqueous solution) to yield the final mask used for etching (Fig. D.3B).

We then performed a conventional top-down anisotropic plasma etch (Unaxis Shuttleline ICP-RIE, $700 \mathrm{~W}$ ICP power, $100 \mathrm{~W}$ RF power, $50 \mathrm{sccm} \mathrm{O}_{2}$ flow rate, $2 \mathrm{sccm}$ Ar flow rate, 10 mTorr chamber pressure) to first transfer the silica etch mask pattern into the diamond to a depth of approximately $600 \mathrm{~nm}$ (Fig. D.3C). We then etched the sample at an angle using the same ICP-RIE parameters as the initial top down etch, but instead housing the sample inside a macroscopic aluminum Faraday cage $[130,195]$ to direct the plasma ions to the substrate surface at the intended angle (Fig. D.3D). Finally, the remaining etch mask was removed in concentrated hydroflu- 
oric acid, followed by 1:3 hydrogen peroxide: conc. sulfuric acid ("piranha") solution leaving freestanding diamond nanocavities (Fig. D.3E).

After the cavities are fabricated, SiV centers are created by targeted implantation using a focused ion beam. This technique will be described in Section D.2.5. Implantation is followed by a tri-acid clean and a three-stage ultra-high-vacuum (maximum pressure $5 \times 10^{-9}$ Torr $)$ anneal at 400 (3 per minute ramp, 8 hour dwell time), 800 (1 per minute ramp, 8 hour dwell time), and 1200 (1 per minute ramp, 4 hour dwell time). This annealing introduces a small amount of graphitic carbon on the surface of the sample. The tri-acid clean is repeated after annealing to remove this carbon. We do not perform a low temperature oxygen anneal. The effect of each post-implantation step is described in more detail in References [54, 205].

\section{D.2.3 Tuning the cavity resonance wavelength}

Fabrication imperfections usually result in cavity resonances that are typically of order $10 \mathrm{~nm}$ away from the desired resonance position. Moreover, cooling the sample to $4 \mathrm{~K}$ introduces additional irreproducible shifts due to thermal contraction of diamond and condensation of residual gas onto the sample surface. Thus, it is highly desirable to have a method to tune the resonance frequency of the cavity in situ during the course of our experiment.

To accomplish this goal, we deliberately introduce a controlled amount of inert gas into the chamber. This gas freezes on the surface of the sample, increasing the local refractive index and shifting the resonance frequency of the cavity to longer wavelengths. Using this technique, we can tune the cavity resonance by more than 
$20 \mathrm{~nm}$ with no deterioration of the cavity quality factor.

Thermal desorption of the tuning gas provides a simple technique to reverse the tuning. Either the sample can be heated up locally using an intense laser field, and/or the whole sample can be heated using a resistive heater mounted on the sample stage. The tuning procedure is very robust and was implemented on the same diamond nanophotonic device many times over several months. For our experiment, we use carbon dioxide as the tuning gas which does not desorb under typical experimental conditions but can be desorbed deliberately using the above methods.

\section{D.2.4 Cavity mode characterization using SiV centers}

We estimate the mode volume of the nanobeam cavity experimentally by probing the local photonic density of states, which is in turn measured via the spontaneous emission rate of $\mathrm{SiV}$ centers into the cavity mode. To implement this novel technique, we use a sample with a similar cavity design but a very high density of $\mathrm{SiV}$ centers created by uniform high-density ion implantation (implantation flux $3 \times 10^{11} \mathrm{~cm}^{-2}$, implantation energy $150 \mathrm{keV}$, performed by Innovion corporation). We then tune the cavity as described in the previous section until the ensemble of $\mathrm{SiV}$ centers is on resonance with the fundamental mode of the cavity. Next, we excite the SiV centers in the cavity by scanning over the entire nanostructure with an off-resonant excitation laser at $700 \mathrm{~nm}$ (Ch. 2 in Fig. D.1 and D.4). We measure the fluorescence into the cavity mode via the outcoupling notch at the end of the cavity (Ch. 3). To produce a one-dimensional plot of the mode density along the beam, we integrate along the direction $(z)$ orthogonal to the beam. We then repeat this procedure for the 
second-order cavity mode by tuning the mode into resonance with the SiV ensemble.

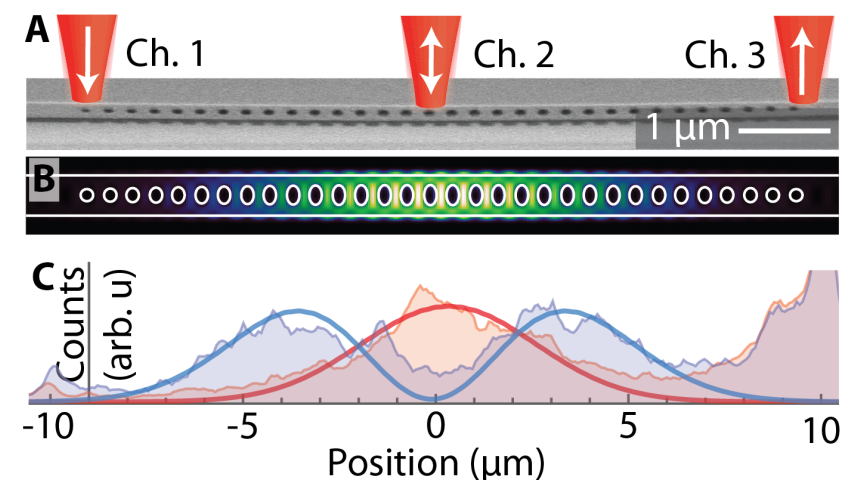

Figure D.4: (A) Scanning electron micrograph of nanophotonic crystal cavity and (B) simulated energy density profile of the cavity mode. (C) Experimentally measured energy density profile for the cavity mode in part B. Photons are detected in the waveguide mode (Ch. 3) as the excitation laser (Ch. 2) is spatially scanned across the nanocavity.

Figure D.4C shows this integrated line cut superimposed with the same integrated line cut taken from our FDTD simulations (see Section D.2.1) where the horizontal axis has been scaled by a fitted parameter to estimate the agreement between simulations and experiment. We assume that the transverse mode profile is given by our simulated value since that mode profile is determined primarily by the size and refractive index of the beam which are well controlled. Before fitting, the simulated data has been convolved with a Gaussian point-spread function $(2 \sigma \approx 600 \mathrm{~nm})$ with width extracted from an image of a single emitter.

We find that the measured mode volume is $35 \%$ larger for the first mode and $46 \%$ larger for the second mode than predicted by our simulations. Under the assumption that this scaling is similar for the devices used in the main text, we estimate an experimental mode volume for the first mode of $V \sim 2.5(\lambda / n)^{3}$ (here, $n=2.4$ ). Discrepancies in the mode volume can result from fabrication imperfections which 
can reduce longitudinal confinement. This mechanism is plausible since we have observed lower quality factors than predicted in our simulations (see Section D.2.1).

\section{D.2.5 Deterministic SiV positioning using focused Si ion beam implantation}

$\mathrm{SiV}$ centers were introduced at the center of the diamond nanocavities by targeted implantation using Sandia National Lab's nanoImplanter, a custom focused-ion-beam system made by the A\&D Corporation (Tokyo). This instrument employs a $100 \mathrm{kV}$ accelerating potential, an $\mathrm{E} \times \mathrm{B}$ mass velocity filter with $m / \Delta m=60$, a laser interferometry controlled stage and a Raith Elphy Plus pattern generator. A AuSiSb liquid metal alloy is used to generate the $\mathrm{Si}^{+}$ion beam. We controlled the ion fluence with a combination of beam current and dwell time at each targeted implantation site. The implantation position was aligned to $<1 \mathrm{~nm}$.

This combination of controlled ion fluence, energy and positioning allows for precise control over the number, depth and lateral position of SiV centers inside the nanophotonic structure. The number of implanted ions obeys a Poisson distribution; the error in the number of implanted ions scales as the square root of the average number of implanted ions. The expected positioning error is dominated by the ion spot size $(40 \mathrm{~nm})$. The range (depth) of the ions is predicted to be $68 \mathrm{~nm}$ with a $\pm 13 \mathrm{~nm}$ straggle. The positioning error is about a factor of two smaller than the relevant cavity mode dimensions: the mode oscillates at about $100 \mathrm{~nm}$ peak-to-peak in the longitudinal direction and has a similar extent in the transverse directions.

Using this approach, we chose an intended dose of between 10 to 500 ions (equiv- 
alent fluence $8 \times 10^{11}$ to $4 \times 10^{13}$ ions $/ \mathrm{cm}^{2}$ ) for different nanocavities on the diamond sample. The nanocavity used in the experiment was implanted with $350 \mathrm{Si}^{+}$ions and contains more than $5 \mathrm{SiV}$ centers (Fig. D.5) after high temperature annealing. These measurements demonstrate greater than $1.5 \%$ conversion yield from $\mathrm{Si}^{+}$ions to $\mathrm{SiV}$ centers, comparable to what has been realized in the bulk[205]. The yield of conversion from $\mathrm{Si}^{+}$ions to $\mathrm{SiV}$ centers is limited by the vacancy density in the diamond crystal. Electron irradiation has been shown to improve the SiV creation yield by more than an order of magnitude[148] and could be used to enable operation with fewer number of implanted ions, reduced damage to the crystal and reduced inhomogeneous distribution.

\section{D.2.6 Adiabatic fiber-waveguide coupling for high-efficiency photon extraction}

For the diamond nano-waveguides used in the Raman tuning and two-SiV entanglement experiments, we collect the Raman emission of $\mathrm{SiV}$ centers by adiabatically transferring the photons from the waveguide mode into a single-mode optical fiber[209]. In this technique, a tapered optical fiber is brought into physical contact with a tapered section of a diamond waveguide. For an appropriate choice of the diamond and fiber geometries, the composite structure supports a single optical mode along its entire length, allowing a photon emitted by a SiV center inside the diamond waveguide to be transfered to the optical fiber. This technique can achieve high absolute coupling efficiencies and requires no realignment over the course of several days. 
To achieve this goal, we use a wet-etching procedure to create conical tapers on conventional single-mode optical fibers (Thorlabs SM600) with a taper angle of approximately 1.5(per side; 3full angle). The triangular diamond waveguide is designed with a similar taper angle of 2 Fiber coupling is achieved by positioning a tapered optical fiber in physical contact with the tapered section of the diamond waveguide using piezoelectric nanopositioners (Attocube ANPx101/ANPz101). The coupling efficiency from single-mode fiber to the diamond waveguide is calculated by sending light into a 90:10 fiber beamsplitter (Thorlabs TW670R5A2), with 90\% of the input light sent to a reference photodiode (Thorlabs PDA100A), and the remaining 10\% to the coupled fiber-waveguide structure. We use a Bragg mirror section of holes (similar to the mirror used to create the photonic crystal cavities above) in the waveguide to reflect the incoming light, $90 \%$ of which is sent to a calibrated measurement photodiode via the final beamsplitter port. By comparing the reflected power with the incoming power, we infer a lower bound on the fiber-diamond coupling efficiency of $70 \%$.

\section{D.3 Identifying single $\mathrm{SiV}$ centers inside nanocav- ities}

The electronic structure of the $\mathrm{SiV}$ center consists of spin-orbit doublets in the ground and excited states. Optical dipole transitions between the doublets result in four transition frequencies which are labeled A-D in order of decreasing frequency[58]. The presence of strain in the crystal changes the energy splittings, resulting in in- 

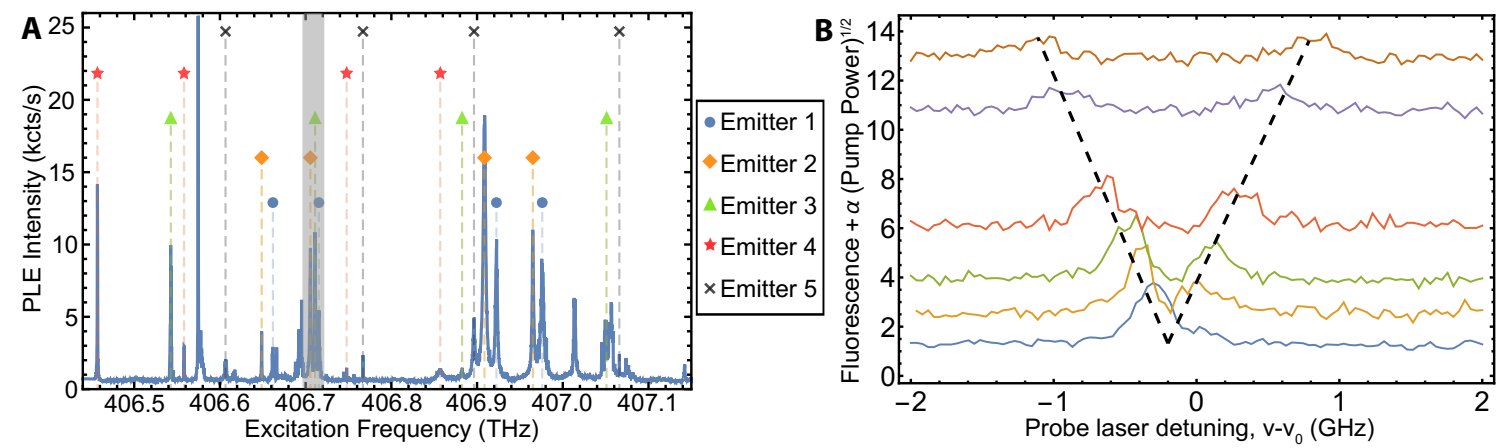

Figure D.5: (A) Fluorescence counts from the center of the cavity as a function of excitation frequency. Colored symbols correspond to emitter frequencies determined by the algorithm described in the text. Emitters 1 and 2 are at low strain, and emitters 3-5 are at higher strain. The shaded region indicates a typical inhomogeneous distribution range of transition $\mathrm{C}(|c\rangle \leftrightarrow|e\rangle)$ frequencies. The cavity QED experiments in the main text were carried out using Emitter 2. (B) The identification procedure was verified by measuring Autler-Townes splitting for the $\Lambda$-type system. A pump laser of varying intensity was fixed on transition $\mathrm{D}(|u\rangle \leftrightarrow|e\rangle)$ at $406.649 \mathrm{THz}$, and a weak probe was scanned across transition $\mathrm{C}$ at $406.705 \mathrm{THz}$. The line splitting on transition $\mathrm{C}$ scales with the square root of the applied power on transition D (illustrated with dashed black line), confirming that the two transitions form an optical lambda system.

homogeneous broadening[128]. However, the difference in the transition frequencies always obeys the energy conservation constraints: $\Delta_{A-B}^{i}=\Delta_{C-D}^{i}=\Delta_{g s}^{i}$ and $\Delta_{A-C}^{i}=\Delta_{B-D}^{i}=\Delta_{e s}^{i}$, where $\Delta_{e s}^{i}$ and $\Delta_{g s}^{i}$ are the excited and ground state splittings, corresponding to an individual emitter labeled $i$.

To determine the set of four transitions corresponding to a single emitter at the center of our cavity, we scanned the Ti:Sapphire laser over a $700 \mathrm{GHz}$ range centered around $406.8 \mathrm{THz}$ at $\sim 100 \mathrm{MHz}$ resolution. We recorded the fluorescence counts in the PSB as a function of excitation frequency to obtain a list of all emitter resonances in this cavity. The resulting spectrum (Fig. D.5A) indicates that there are several near-resonant $\mathrm{SiV}$ centers at the center of the cavity where each $\mathrm{SiV}$ results in four 
lines $\mathrm{A}^{i}-\mathrm{D}^{i}$. We fit the frequency of each line in the Fig. D.5A and calculate the energy differences $\Delta$ between all pairs of lines. From this list, we find sets of four frequencies $\mathrm{A}^{i}-\mathrm{D}^{i}$ that consist of pairs of transitions with matching $\Delta$, i.e. $\left|\Delta_{A-B}^{i}-\Delta_{C-D}^{i}\right|<\epsilon$ where $\epsilon \sim 300 \mathrm{MHz}$ is limited by experimental error in frequency estimation.

The results of this approach are indicated in Fig. D.5A where we have drawn colored symbols over the sets of lines presumably corresponding to the same SiV. (Only five emitters are labeled.) To verify that these lines correspond to the same SiV, we apply a strong driving field on transition $\mathrm{D}^{i}\left(\mathrm{~B}^{i}\right)$ and probe the response on transition

$\mathrm{C}^{i}\left(\mathrm{~A}^{i}\right)$. We observe that the probed transition undergoes a splitting proportional to the square root of the power of the driving field (Fig. D.5B). This signature of the Autler-Townes effect confirms that the two transitions share an excited state as expected. When the two lines do not correspond to the same $\mathrm{SiV}$, there is no observable splitting. For the optical switch experiment shown in Fig. 2 of the main manuscript, transitions $\mathrm{C}(|c\rangle \leftrightarrow|e\rangle)$ and $\mathrm{D}(|u\rangle \leftrightarrow|e\rangle)$ were used to realize an optical lambda system.

\section{D.4 SiV charge state control: high fidelity initial- ization and single-shot readout}

The $\mathrm{SiV}$ can be occasionally ionized from the $\mathrm{SiV}^{-}$charge state to other charge states $\left(\mathrm{SiV}^{0}\right.$ or $\left.\mathrm{SiV}^{2-}\right)$ which are dark in our measurement scheme. It is highly desirable to know when the $\mathrm{SiV}$ is in the correct charge state and to actively control the charge state. To accomplish these tasks, we performed the cavity transmission 
experiment in Fig. 1D with both the cavity and emitter on resonance with the probe laser. At first, only the weak, resonant probe laser was used for driving the system.

We recorded both the transmission photons collected through the cavity and the fluorescence photons collected in the phonon-sideband (PSB), and binned them in $15 \mathrm{~ms}$ intervals as shown in the blue curves in Fig. D.6A. The SiV jumps between a bright state $\left(\mathrm{SiV}^{-}\right)$where the transmission intensity (thick blue trace) is low and the PSB fluorescence intensity (thin blue trace) is high and a dark state (not coupled to the probe field) with high transmission and no fluorescence. These jumps are clearly resolved on the timescale of seconds, allowing single-shot readout of the charge state of the SiV.

To rule out the possibility of faster ionization dynamics that we could not resolve with this slow technique, we performed a similar experiment but recorded the timetagged photon arrival times with the fast acquisition electronics described above. We observed no features slower than tens of nanoseconds, indicating that the complete electronic dynamics are limited to those of the three-level optical system plus a slow charge switching process at the timescale of seconds for the laser intensities used in the experiment.

We then repeat the first experiment, with the addition of a $10 \mu$ s pulse of around $0.4 \mathrm{~mW}$ green light $(520 \mathrm{~nm})$ every $100 \mu \mathrm{s}$. The detectors are gated off during the green pulse. The timetraces recorded with this scheme (and with the same duty cycle and data processing as above) are shown in the green timetraces (thick: transmission, thin: PSB fluorescence) in Fig. D.6A. These traces are flat and close to the values when the $\mathrm{SiV}$ is in the correct charge state, illustrating that there are no dynamics on 

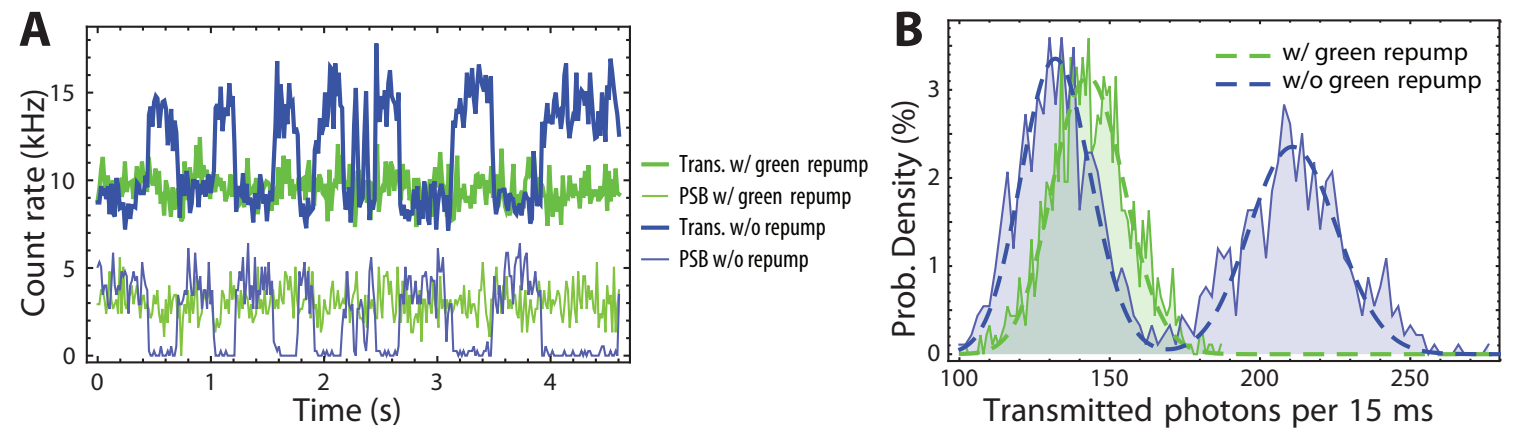

Figure D.6: Charge state initialization with and without a green repump pulse. (A) Timetraces of transmission (upper thick curves) and phonon sideband (PSB, lower thin curves) photon detection events as a function of time. The data are taken both with (green) and without (blue) the presence of a $520 \mathrm{~nm}$ repump laser at approximately $10 \%$ duty cycle. Clear jumps are present in the case of no green repump, indicating single-shot readout of charge-state fluctuations. (B) Histograms of timetraces in A with Poissonian fits. The ratio of the two blue peak positions gives a direct measurement of the transmission dip of $37 \%$. The mean number of transmitted photons in the case with green repump can be decomposed into $85 \%$ of the low-transmission peak and $15 \%$ of the high transmission peak in the case of no green repump. This implies an $85 \%$ charge state initialization fidelity.

the millisecond timescale and that the green pulse effectively repumps the SiV into the desired charge state.

To quantify this effect, we make histograms of photons detected per $15 \mathrm{~ms}$ bin for the transmission counts both with and without green repump (Fig. D.6B). We fit a Poisson distribution to the histogram of counts taken with green repump and two Poisson distributions to the histogram of counts taken with no repump. Based on the relative amplitude of the two peaks without green repump, we estimate that the $\mathrm{SiV}$ is in the correct charge state $53 \%$ of the time. The average counts for the data taken with the green repump pulse can be decomposed into $85 \%$ of the low-transmission ( $\mathrm{SiV}$ on) case and $15 \%$ of the high-transmission ( $\mathrm{SiV}$ off) case, implying a chargestate initialization fidelity of $85 \%$. A similar analysis using the PSB fluorescence has 
more noise but is consistent with the above estimate. The close fit of these Poisson distributions to our data also indicates that there is no significant noise above shot noise on this timescale.

Finally, by measuring the contrast between the transmission counts in the bright and dark cases (i.e. the mean values of the peaks in Fig. D.6B) we also have a simple and direct measurement of the transmission extinction from a single SiV. The value of the transmission dip extracted from this measurement is $37 \%$, consistent with the measurement in Fig. 1F of the main manuscript. While the single-shot readout of the charge state shown in Fig. D.6 can be used to initialize the system with near-perfect fidelity, further research is necessary to understand the microscopic mechanism for charge dynamics and develop higher fidelity control techniques.

\section{D.5 Model description for a $\mathrm{SiV}$ center inside an optical cavity}

In this section, we describe the theoretical model used to describe the dynamics of the SiV-cavity system measured in Figs. 7.1-7.3 of Chapter 7. We show how to calculate the saturation response of the transmission and fluorescence of the system (Fig. 7.3A) and the different intensity correlation functions between detection events (Figs. 3B-D). 


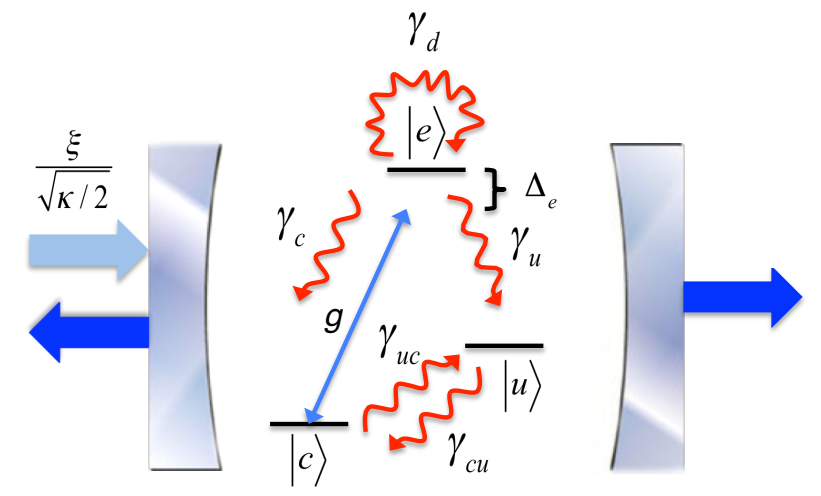

Figure D.7: Setup for the coupled SiV-cavity system with dissipation. $\xi / \sqrt{\kappa / 2}$ is the flux amplitude of the weak coherent probe field, and $\kappa$ is the total decay rate of the symmetric cavity. Dissipation channels are characterized by decay rates $\gamma$. The cavity drives the $|c\rangle \leftrightarrow|e\rangle$ transition with a single-photon Rabi frequency $g$ and a detuning $\Delta_{e}$.

\section{D.5.1 Three level dynamics with dissipation}

The system is modeled by a three level atom inside a driven cavity as depicted in Fig. D.7. In a frame rotating with the probe frequency, the Hamiltonian is

$$
\hat{H}=\Delta_{e}|e\rangle\langle e|+\Delta_{c} \hat{a}^{\dagger} \hat{a}+i \xi\left(\hat{a}^{\dagger}-\hat{a}\right)+i g\left(\hat{a}|e\rangle\left\langle c\left|-\hat{a}^{\dagger}\right| c\right\rangle\langle e|\right)
$$

where $\Delta_{e}=\omega_{e c}-\omega_{p}, \Delta_{c}=\omega_{c}-\omega_{p}, \omega_{e c}$ is the transition frequency between level $|e\rangle$ and $|c\rangle$, and $\omega_{c}$ is the resonance frequency of the cavity. The coupling between the cavity field and the $\mathrm{SiV}$ is given by the single-photon Rabi frequency $g$. The driving probe field is in a weak coherent state with a flux amplitude $\xi / \sqrt{\kappa / 2}$ and frequency $\omega_{p}$. The bosonic annihilation operator $\hat{a}$ describes the cavity field. The dissipation in 
the system is described by the Lindblad operators

$$
\begin{array}{ll}
\hat{L}_{1}=\sqrt{\gamma_{c}}|c\rangle\langle e|, & \hat{L}_{4}=\sqrt{\gamma_{u c}}|u\rangle\langle c|, \\
\hat{L}_{2}=\sqrt{\gamma_{u}}|u\rangle\langle e|, & \hat{L}_{5}=\sqrt{\gamma_{d}}|e\rangle\langle e|, \\
\hat{L}_{3}=\sqrt{\gamma_{c u}}|c\rangle\langle u|, & \hat{L}_{6}=\sqrt{\kappa} \hat{a}
\end{array}
$$

where $\gamma_{u c}$ and $\gamma_{c u}$ are the nonradiative decay rates between states $|c\rangle$ and $|u\rangle, \gamma_{c}\left(\gamma_{u}\right)$ is the decay rate from state $|e\rangle$ to state $|c\rangle(|u\rangle), \gamma_{d}$ is the dephasing rate of state $|e\rangle$ and $\kappa$ is the total decay rate of the cavity field. The Markovian approximation for the nonradiative relaxation rates $\left(\gamma_{u c}, \gamma_{c u}, \gamma_{d}\right)$ is justified by recent measurements of dephasing $\left(\gamma_{d}\right)$ and depolarization $\left(\gamma_{c u}\right)$ induced by a thermal phonon bath[181]. Experimentally, we find that the optical transitions can be well described by Lorentzian profiles (see Fig. 7.1F), and that the slow non-Markovian dephasing (spectral diffusion) has a small contribution to the total linewidth.

The master equation describing the atom-cavity system can be written formally as

$$
\dot{\rho}=-i[\hat{H}, \rho]+\sum_{x=1}^{6} \hat{L}_{x} \rho \hat{L}_{x}^{\dagger}-\frac{1}{2}\left(\hat{L}_{x}^{\dagger} \hat{L}_{x} \rho+\rho \hat{L}_{x}^{\dagger} \hat{L}_{x}\right)
$$

We work in the weak driving regime $(\xi \ll \kappa)$ and can therefore truncate the Hilbert space. To lowest order, we assume that at most two excitations are present in the system such that we can describe the system in the basis

$$
\{|0, c\rangle,|0, e\rangle,|0, u\rangle,|1, c\rangle,|1, e\rangle,|1, u\rangle,|2, c\rangle,|2, u\rangle\} .
$$

We label these states $\{|1\rangle \ldots|8\rangle\}$. 


\section{D.5.2 System saturation response}

To model the saturation response of the transmission and fluorescence measured in Fig. 7.3A, we look at the steady state of the system. For this calculation, we assume that the cavity is driven resonantly $\left(\Delta_{c}=0\right)$ and we are in the regime where $\kappa \gg g, \Delta_{e}, \xi, \gamma$ and $g \gg \gamma$. This makes it possible to approximately solve the master equation for the steady state density matrix elements $\rho_{i, j}^{(s)}=\left\langle i\left|\rho^{(s)}\right| j\right\rangle$ analytically. The expressions for $\rho_{i, j}^{(s)}$ can be found in Sec. D.9.1.

The transmission is then given by

$$
\mathcal{T}=\frac{\kappa_{b}\left\langle\hat{a}^{\dagger} \hat{a}\right\rangle_{s}}{\left\langle\hat{a}_{\mathrm{in}}^{\dagger} \hat{a}_{\mathrm{in}}\right\rangle} \approx \frac{\kappa_{a} \kappa_{b}\left(\rho_{4,4}^{(s)}+\rho_{6,6}^{(s)}\right)}{\xi^{2}},
$$

where $\kappa_{a}\left(\kappa_{b}\right)$ describes the cavity decay rate to the input (output) waveguide mode. The total cavity decay rate is $\kappa=\kappa_{a}+\kappa_{b}+\kappa^{\prime}$, where $\kappa^{\prime}$ is the loss rate out of the cavity not collected by the input and output waveguide modes. In our experiment, we use a waveguide damped symmetric cavity with $\kappa_{a} \approx \kappa_{b} \approx \kappa / 2 .\left\langle\hat{a}_{\text {in }}^{\dagger} \hat{a}_{\text {in }}\right\rangle=2 \xi^{2} / \kappa$ is the photon flux of the input coherent state. The fluorescence scattering is given by

$$
\mathcal{F}=\frac{\left\langle\sigma_{e, e}\right\rangle_{s}}{\left\langle\hat{a}_{\text {in }}^{\dagger} \hat{a}_{\text {in }}\right\rangle} \approx \frac{\left(\gamma_{c}+\gamma_{u}\right) \kappa}{2 \xi^{2}}\left(\rho_{2,2}^{(s)}+\rho_{5,5}^{(s)}\right)
$$

where $\hat{\sigma}_{e, e}=|e\rangle\langle e|$. We note that the observed saturation response differs from the two-level system response due to the presence of a second metastable level $|u\rangle$.

These expressions for $\mathcal{T}$ and $\mathcal{F}$ successfully capture the experimentally measured saturation response in fluorescence and transmission (see Fig. 7.3A) using the system parameters given in Section D.5.4. In Fig. 7.3A, we express the photon flux, $2 \xi^{2} / \kappa$, in terms of number of photons per Purcell-reduced excited-state lifetime $\tau_{e}$. The experimental data (dots) were fit to the theoretical curves (solid curves) using a 
single fit parameter for the scaling between the photon flux at the cavity (horizontal scale in the figure) and the photon detection rate. We find that a photon detection rate of approximately $110 \mathrm{kHz}$ corresponds to a photon flux of 1 photon per lifetime. This scaling parameter accounts for the detection efficiency in the experiment, and the fit result is in agreement with our independent estimates of the detection efficiency $\left(\sim 10^{-4}\right)$.

\section{D.5.3 Calculation of intensity correlation functions}

In order to calculate the intensity correlation functions measured in Fig. 7.3, we need to evaluate the response of the system following the detection of either a cavity photon or a scattered photon. Since we work below saturation, the evolution of the metastable states $(|0, c\rangle,|0, u\rangle)$ are approximately determined solely by the dissipative coupling between these two states such that

$$
\begin{aligned}
& \dot{\rho}_{1,1} \approx \gamma_{c u} \rho_{3,3}-\gamma_{u c} \rho_{1,1} \\
& \dot{\rho}_{3,3} \approx \gamma_{u c} \rho_{1,1}-\gamma_{c u} \rho_{3,3} .
\end{aligned}
$$

This can be solved to yield $\rho_{1,1}(t)=\alpha+\beta e^{-\tilde{\gamma} t}, \rho_{3,3}(t)=\alpha_{2}-\beta e^{-\tilde{\gamma} t}$, where $\alpha=$ $\frac{\gamma_{c u}\left(\rho_{1,1}(0)+\rho_{3,3}(0)\right)}{\tilde{\gamma}}, \beta=\frac{\gamma_{u c} \rho_{1,1}(0)-\gamma_{c u} \rho_{3,3}(0)}{\tilde{\gamma}}, \alpha_{2}=\frac{\gamma_{u c}\left(\rho_{1,1}(0)+\rho_{3,3}(0)\right)}{\tilde{\gamma}}$ and $\tilde{\gamma}=\gamma_{c u}+\gamma_{u c}$. In steady state, the populations are given by $\rho_{1,1}^{(s)} \approx \gamma_{c u} /\left(\gamma_{c u}+\gamma_{u c}\right)$ and $\rho_{3,3}^{(s)} \approx \gamma_{u c} /\left(\gamma_{c u}+\right.$ $\left.\gamma_{u c}\right)$. The ratio of $\gamma_{u c} / \gamma_{c u}$ is determined by the thermal distribution[181]. Using data from the optical pumping experiment shown in Fig. 7.2, we infer $\rho_{1,1} \sim 64 \%$ and $\rho_{3,3} \sim 36 \%$ in steady state. These values are in good agreement with a thermal distribution of population at $4 \mathrm{~K}$ for the two metastable states that are split by $64 \mathrm{GHz}$. 
Using these expressions for the metastable state populations, we can analytically solve the equations of motion for the populations in the other levels to the leading order in the probe field amplitude. We use the assumption of weak resonant driving $\left(\Delta_{c}=0\right)$ and that we are in the bad cavity regime $(\kappa \gg g \gg \gamma \gg \xi$, ) to identify the dominant terms and adiabatically eliminate all states containing cavity photons. As a result, we are left with only three coupled differential equations that we need to solve between $\dot{\rho}_{2,2}$ and $\dot{\rho}_{1,2}, \dot{\rho}_{2,1}$. Solving the equations, we obtain the following expressions:

$$
\begin{aligned}
\rho_{1,2}(t) & \approx \mathcal{A}_{1}+\mathcal{A}_{2} e^{-\tilde{\gamma} t}+\mathcal{A}_{3} e^{a_{1} t}, & \rho_{6,6}(t) & \approx \frac{4 \xi^{2}}{\kappa^{2}} \rho_{3,3}(t), \\
\rho_{2,2}(t) & \approx \mathcal{B}_{1}+\mathcal{B}_{2} e^{-\tilde{\gamma} t}+\mathcal{B}_{3} e^{a_{1} t}+\mathcal{B}_{4} e^{b_{1} t}, & \rho_{7,7}(t) & \approx \frac{4\left(\xi^{2} \rho_{4,4}(t)+g^{2} \rho_{5,5}(t)\right)}{3 \kappa^{2}} \\
\rho_{4,4}(t) & \approx \mathcal{C}_{1}+\mathcal{C}_{2} e^{-\tilde{\gamma} t}+\mathcal{C}_{3} e^{a_{1} t}+\mathcal{C}_{4} e^{b_{1} t}, & \rho_{8,8}(t) & \approx \frac{6 \xi^{2}}{3 \kappa^{2}} \rho_{6,6}(t), \\
\rho_{5,5}(t) & \approx \frac{4 \xi^{2}}{\kappa^{2}} \rho_{2,2}(t) . & &
\end{aligned}
$$

The coefficients $a_{i}, b_{i}, \mathcal{A}_{i}, \mathcal{B}_{i}$ and $\mathcal{C}_{i}$ are defined in Sec. D.9.2.

From Eqs. (D.9)-(D.12), it is straightforward to get the steady state population of the different levels by taking the limit $t \rightarrow \infty$. From the steady state populations we can calculate $\left\langle\hat{a}^{\dagger} \hat{a}\right\rangle \approx \rho_{4,4}^{(s)}+\rho_{6,6}^{(s)}$ and $\left\langle\hat{\sigma}_{e, e}\right\rangle \approx \rho_{2,2}^{(s)}$. The normalized transmissiontransmission intensity correlation function (Fig. 7.3C) is given by

$$
g_{T T}^{(2)}(\tau)=\frac{\left\langle\hat{a}^{\dagger}(0) \hat{a}^{\dagger}(\tau) \hat{a}(\tau) \hat{a}(0)\right\rangle}{\left\langle\hat{a}^{\dagger} \hat{a}\right\rangle^{2}},
$$

where $\left\langle\hat{a}^{\dagger}(0) \hat{a}^{\dagger}(\tau) \hat{a}(\tau) \hat{a}(0)\right\rangle \approx \rho_{4,4}(\tau)+\rho_{6,6}(\tau)$ can be calculated from Eqs. (D.9) and (D.11) with initial conditions given by the detection of a cavity photon at time $t=0$, i.e. $\rho(0)=\hat{a} \rho^{(s)} \hat{a}^{\dagger}$. Consequently, $\rho_{1,1}(0)=\rho_{4,4}^{(s)}, \rho_{3,3}(0)=\rho_{6,6}^{(s)}, \rho_{2,2}(0)=\rho_{5,5}^{(s)}$ and $\rho_{1,2}(0)=\left(4 \xi^{2} \rho_{1,2}^{(s)}+2 \xi g \rho_{4,4}^{(s)}-4 \xi g \rho_{2,2}^{(s)}\right) / \kappa^{2}$. The normalized intensity autocorrelation 
function for the scattered (fluorescence) field (see Fig. 7.3B)

$$
\begin{aligned}
g_{S S}^{(2)}(\tau)= & \frac{\gamma_{u}^{(r a d)}}{\gamma_{c}^{(r a d)}+\gamma_{u}^{(r a d)}} \frac{\left\langle\hat{\sigma}_{e, u}(0) \hat{\sigma}_{e, e}(\tau) \hat{\sigma}_{u, e}(0)\right\rangle}{\left\langle\hat{\sigma}_{e, e}\right\rangle^{2}} \\
& +\frac{\gamma_{c}^{(r a d)}}{\gamma_{c}^{(r a d)}+\gamma_{u}^{(r a d)}} \frac{\left\langle\hat{\sigma}_{e, c}(0) \hat{\sigma}_{e, e}(\tau) \hat{\sigma}_{c, e}(0)\right\rangle}{\left\langle\hat{\sigma}_{e, e}\right\rangle^{2}}
\end{aligned}
$$

and the normalized intensity correlation function between the scattered and transmitted fields (see Fig. 7.3D)

$$
\begin{aligned}
g_{S T}^{(2)}\left(\tau=\tau_{F}-\tau_{T}>0\right)= & \frac{\left\langle\hat{a}^{\dagger}(0) \hat{\sigma}_{e, e}(\tau) \hat{a}(0)\right\rangle}{\left\langle\hat{a}^{\dagger} \hat{a}\right\rangle\left\langle\hat{\sigma}_{e, e}\right\rangle} \\
g_{S T}^{(2)}(\tau<0)= & \frac{\gamma_{u}^{(r a d)}}{\gamma_{c}^{(r a d)}+\gamma_{u}^{(r a d)}} \frac{\left\langle\hat{\sigma}_{e, u}(0) \hat{a}^{\dagger}(-\tau) \hat{a}(-\tau) \hat{\sigma}_{u, e}(0)\right\rangle}{\left\langle\hat{a}^{\dagger} \hat{a}\right\rangle\left\langle\hat{\sigma}_{e, e}\right\rangle} \\
& +\frac{\gamma_{c}^{(r a d)}}{\gamma_{c}^{(r a d)}+\gamma_{u}^{(r a d)}} \frac{\left\langle\hat{\sigma}_{e, c}(0) \hat{a}^{\dagger}(-\tau) \hat{a}(-\tau) \hat{\sigma}_{c, e}(0)\right\rangle}{\left\langle\hat{a}^{\dagger} \hat{a}\right\rangle\left\langle\hat{\sigma}_{e, e}\right\rangle}
\end{aligned}
$$

can be calculated in a similar way where $\hat{\sigma}_{u, e}=|u\rangle\langle e|$ and $\hat{\sigma}_{c, e}=|c\rangle\langle e|$. Equations (D.15) and (D.16) correspond to cases where a transmitted or a scattered photon was detected first, respectively. Note that for correlations involving the fluorescence field, detection of photons emitted in $|e\rangle \rightarrow|u\rangle$ (detection probability $\sim \gamma_{u}^{(\mathrm{rad})}$ ) or $|e\rangle \rightarrow|c\rangle$ (detection probability $\sim \gamma_{c}^{(r a d)}$ ) can result in different dynamics and two separate terms. While these photons (or two paths) are in principle distinguishable by frequency, in our experiment both paths were detected without frequency filtering. For this reason, a photon detection in fluorescence leaves the $\mathrm{SiV}$ in some classical mixture of states $|c\rangle$ (with probability $\gamma_{c}^{(\mathrm{rad})} /\left(\gamma_{c}^{(\mathrm{rad})}+\gamma_{u}^{(\mathrm{rad})}\right)$ ) and $|u\rangle$ (with probability $\left.\gamma_{u}^{(\mathrm{rad})} /\left(\gamma_{c}^{(\mathrm{rad})}+\gamma_{u}^{(\mathrm{rad})}\right)\right)$. On the other hand, the detection of a cavity photon preferentially leaves the $\mathrm{SiV}$ in state $|u\rangle$. This results in the asymmetry in the fluorescence-transmission intensity cross-correlation function measured in Fig. 7.3D. The observed dynamics in photon correlations in Fig. 7.3, can be understood based 
on these multivel dynamics. Upon detection of a transmitted photon, the $\mathrm{SiV}$ has an increased likelihood to be projected into state $|u\rangle$, where the $\mathrm{SiV}$ is not excited by laser light. Consequently, for a time period $\left(\sim \tau_{0}\right)$ given by the lifetime of state $|u\rangle$, the SiV-cavity system will have higher transmission and reduced scattering resulting in

enhanced $g_{T T}^{(2)}$ and suppressed $g_{S T}^{(2)}$. If instead a scattered photon is detected first, the $\mathrm{SiV}$ is preferentially projected to the coupled state $|c\rangle$. This state undergoes two-level dynamics associated with decreased scattering and enhanced transmission at short times on the order of the excited state lifetime $\tau_{e}$. At longer times of order $\tau_{0}$ while the system is more likely to remain in the coupled state $|c\rangle$, it exhibits enhanced scattering $\left(g_{S S}^{(2)}\right.$, Fig. $\left.7.3 \mathrm{~A}\right)$ and somewhat reduced transmission $\left(g_{S T}^{(2)}\right.$, Fig. $\left.7.3 \mathrm{C}\right)$. These results are also consistent with the optical switching dynamics in Fig. 2 where the system is polarized using a classical gate pulse instead of a single photon detection event and the relaxation dynamics at the same timescale $\tau_{0}$ are observed.

\section{D.5.4 Extraction of system parameters}

The data shown in Figs. 7.1-7.3 of the main manuscript can be modeled using a single set of intrinsic system parameters. The cavity decay rate $\kappa$ is measured from the envelope of the cavity transmission curve in Fig. 7.1E (blue curve). The dephasing rate $\gamma_{d}$ of the $\mathrm{SiV}$ optical transition $(|c\rangle \rightarrow|u\rangle)$ is measured from the $\mathrm{SiV}$ linewidth in Fig. $7.1 \mathrm{~F}$ when the cavity is off resonance with the $\mathrm{SiV}$ (orange curve), while the single-photon Rabi frequency $g$ is determined by the Purcell-broadened linewidth when the cavity is on resonance with the $\mathrm{SiV}$ (red curve). These values are consistent with the independent time-domain lifetime measurement described in the main text. 
These values (which suffice to determine the cooperativity) are then taken as fixed; the remaining parameters are extracted from the photon correlation measurements in Figs. 7.3C and 7.3D. (The data in Fig. 7.3B were taken above saturation with large cavity detuning $\left(\Delta_{e}=0, \Delta_{c}>>\kappa\right)$ and were modeled separately as a threelevel atom in a classical field.) The optical switching experiment (Fig. 7.2) provides an independent measurement of the ground-state relaxation rates $\gamma_{u c}$ and $\gamma_{c u}$ and is consistent with the values extracted from the photon correlation measurements.

The following parameters (defined in Fig. D.7) accurately describe the system dynamics: $\left\{\kappa=1150 \gamma_{0}, \gamma_{d}=4 \gamma_{0}, \gamma_{u}=\gamma_{0}, \gamma_{c}=\gamma_{0}, \gamma_{u c}=0.10 \gamma_{0}, \gamma_{c u}=0.15 \gamma_{0}, g=\right.$ $\left.\gamma_{0}\left(1.5 \kappa / \gamma_{0}\right)^{1 / 2}, \gamma_{c}^{(\text {rad })} / \gamma_{u}^{(r a d)}=2\right\}$ where we defined $\gamma_{0}=2 \pi \times 50 \mathrm{MHz}$. Of these parameters, $\left\{\kappa, \gamma_{d}, \gamma_{u c}, \gamma_{c u}, g\right\}$ are consistent with independently measured values in

Figs. 7.1-7.3. The detected ratio of $\gamma_{c}^{(r a d)} / \gamma_{u}^{(r a d)}$ determines the asymmetry of $g_{S T}^{2}(\tau)$ and was fit separately using the data in Fig. 7.3D. The theoretical expressions for photon correlations were convolved with the detector timing response (300 ps timing jitter per APD) to obtain the solid curves in Fig. 7.3. Intensity correlation measurements were normalized using intensity averages; we do not observe excess noise due to incoherent dynamics at slow timescales.

\section{D.5.5 Measured vs. expected cooperativity}

Our measured cooperativity of $C \sim 1$ differs substantially from the cooperativity $C=\frac{4 g^{2}}{\kappa \gamma}=\frac{3}{4 \pi^{2}} \frac{Q}{V}\left(\frac{\lambda}{n}\right)^{3} \sim 275$ in the ideal case of a perfect emitter coupled optimally to our cavity with measured quality factor $Q \sim 7200$ and dimensionless mode volume $\frac{V}{(\lambda / n)^{3}} \sim 3$. In this section, we discuss the discrepancies leading to this disagreement. 
First, the emitter is not optimally aligned with the cavity mode. Based on the crystallographic orientation of our sample and the electric field mode profile inside the cavity, the maximal overlap between the atomic transition dipole and the cavity mode is roughly $2 / 3$. The positioning of the $\mathrm{SiV}$ center can be a separate source of error. The accuracy of FIB-based implantation (Section D.2.5) is around $40 \mathrm{~nm}$, including straggle. This is a significant fraction of the spatial extent of the local cavity field antinode that extends in about $100 \mathrm{~nm}$ in all three dimensions. This positioning error can reduce the atom-photon coupling and hence the cooperativity by roughly $20-40 \%$.

In addition to reductions in the cooperativity due to emitter positioning errors, the $\mathrm{SiV}$ is not a perfect optical emitter. The atomic decay rate $\gamma$ in the cooperativity includes all photon emission and decoherence rates, whereas the single-photon Rabi frequency $g$ includes only the contribution from the dipole transition $(|c\rangle \leftrightarrow|e\rangle)$ that couples to the cavity mode. The cooperativity is therefore reduced by imperfections in the optical transitions of the SiV center. First, the Debye-Waller factor $\left(\gamma_{Z P L}^{(r a d)} / \gamma^{(r a d)}\right)$ for the SiV ZPL is around 70\%. Next, our linewidth is broadened by phonon processes and spectral diffusion to approximately three times the lifetime-limited linewidth $\left(\gamma_{0} / \gamma_{e x p} \approx 0.3\right)$. For the lambda system used in the experiment, the branching ratio of the $|e\rangle \rightarrow|c\rangle$ and $|e\rangle \rightarrow|u\rangle$ ZPL transitions are about $80 \%$ and $20 \%$ respectively[59]. Finally, if there is a significant nonradiative decay rate limiting the quantum efficiency (QE) of the $\mathrm{SiV}$, our observed cooperativity could be substantially reduced.

Taking conservative estimates for the parameters other than an unknown quantum efficiency, our estimated cooperativity is around $C \sim 10 \times \mathrm{QE}$ (Table D.1). A quantum 


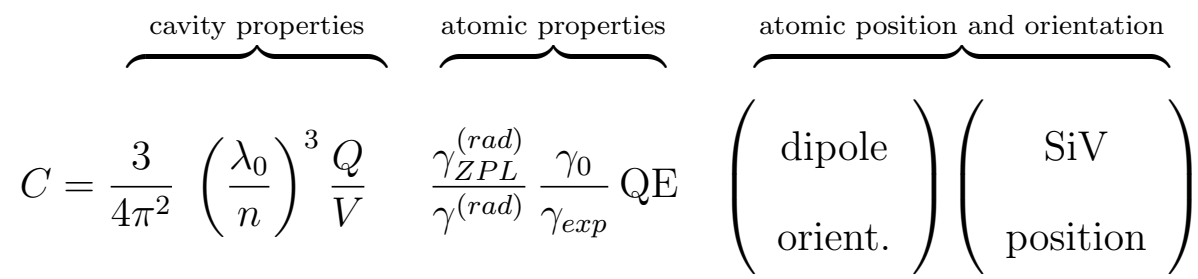

$$
\begin{aligned}
& C \approx \frac{1}{13} \quad \frac{7200}{3} \quad 0.7 \quad 0.3 \mathrm{QE} \quad 0.4-0.8 \quad 0.4-1 \quad \sim 10 \times \mathrm{QE}
\end{aligned}
$$

Table D.1: Cooperativity estimate based on $\mathrm{SiV}$ and experimental parameters described in the text

efficiency of around $10 \%$ is therefore consistent with the discrepancy between our expected and observed cooperativity.

\section{D.6 Subnatural-linewidth Raman single photons}

The Raman tuning demonstrated in Fig. 7.4 uses off-resonant laser excitation to generate spectrally-tunable subnatural linewidth single photons. In this section, we discuss technical considerations that enable us to isolate single photons under off-resonant excitation and present data that support our claims about subnatural linewidths for Raman photons. The $\mathrm{SiV}$ centers used for the Raman tuning and entanglement experiments have a ground state frequency splitting of $\approx 50 \mathrm{GHz}$. When we excite the $|u\rangle \rightarrow|e\rangle$ transition with a laser at frequency $\nu$, the Raman photons are at a frequency $\approx \nu+50 \mathrm{GHz}$. We use separate spatial modes for laser excitation (free-space, see Fig. 7.4) and Raman photon detection (waveguide mode collected via fiber). This allows us to significantly suppress leakage from the excitation laser into our collection mode. Despite this suppression, the scattering at the diamondvacuum interface results in laser leakage into the collection mode. When we excite 
the $|u\rangle \rightarrow|e\rangle$ transition on resonance, we find that the typical signal to noise ratio between the Raman single-photons and laser background is about $\sim 1$.

To obtain Raman single-photons with high purity, we use a home-built scanning Fabry-Perot (FP) filter cavity designed to have high transmission for Raman photons at frequency $\approx \nu+50 \mathrm{GHz}$ while suppressing laser photons at frequency $\nu$. To achieve this, we built a cavity with a free spectral range (FSR) of $37 \mathrm{GHz}$ which maximally suppresses the laser field (by putting the laser line in the middle of the FSR, exactly between two cavity resonances) while maintaining a high cavity bandwidth of $150 \mathrm{MHz}$ (FWHM, ringdown time $1.05 \mathrm{~ns}$ ). We note that the high bandwidth is necessary to be able to measure the dynamics observed at the $\approx 2.5 \mathrm{~ns}$ timescale in Fig. $7.5 \mathrm{D}$. The fit functions used for the data in Fig. 7.5D are convolved with the combined timing response of the cavity and the detectors.

The use of the FP cavity allows us to obtain a signal to noise ratio of $\sim 1000$ under resonant excitation. At increased detunings $\Delta$, the scattering cross section of the $\mathrm{SiV}$ reduces according to $\sim \frac{1}{(\Gamma / 2)^{2}+\Delta^{2}}$, where $\Gamma$ is the $|u\rangle \leftrightarrow|e\rangle$ transition linewidth. This scaling is confirmed with the measurements shown Fig. D.8A where we measure the Raman emission intensity at different detunings for a fixed laser power. The reduced scattering cross section at large detunings necessitates an increased laser power to maintain a fixed photon scattering and detection rate. The extinction ratio of the FP cavity ( $\sim 10^{4}$ for current device) is therefore a crucial parameter that sets the practical limit on the extent of Raman tuning. With the cavity used in the experiments, we are able to tune the Raman photons by $10 \mathrm{GHz}$ in each direction while maintaining a signal to noise ratio above 1 (see extended data in Fig. D.8). The tuning range 
could be further extended by using a higher finesse cavity (or a second filter cavity) or cavity-enhanced Raman scattering.
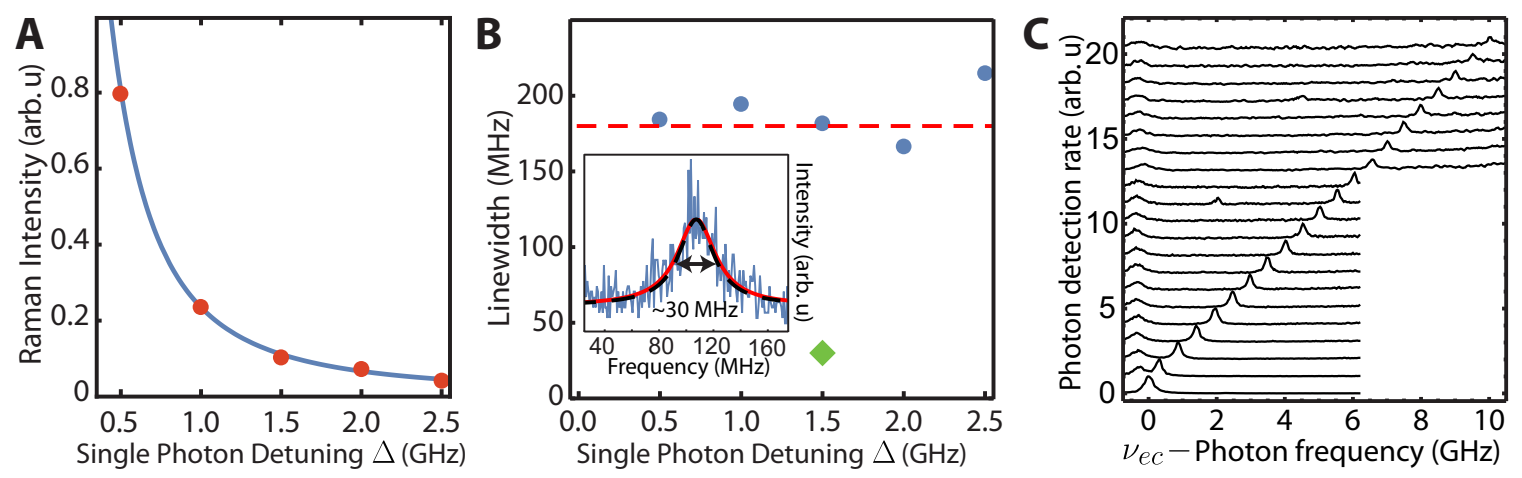

Figure D.8: (A) Raman fluorescence intensity for a fixed laser power measured at different single photon detunings $\Delta$. The solid line is a fit with the form $\frac{A}{(\Gamma / 4 \pi)^{2}+(\Delta)^{2}}$ with $\Gamma / 2 \pi=440 \mathrm{MHz}$. (B) Raman emission linewidth as a function of detuning. At all detunings, the Raman emission linewidth (blue circles) is limited by the cavity linewidth (dashed red line). When a narrow-linewidth cavity with FWHM $\sim 30 \mathrm{MHz}$ is used to measure the Raman linewidth, the linewidth is significantly narrower (green diamond). Inset: Raman fluorescence intensity as a function of narrow-linewidth cavity frequency. The fitted Lorentzian response (solid red curve) is almost identical to the measured cavity transfer function (dashed black curve) indicating that the Raman linewidth is less than the $30 \mathrm{MHz}$ cavity bandwidth. (C) Extended data for Fig. 7.4C, showing Raman tuning over $10 \mathrm{GHz}$ on the red sideband.

Under off-resonant excitation with $\Omega, \Gamma<<\Delta$, the linewidth of the Raman photons is determined by the coherence between the metastable states $|u\rangle$ and $|c\rangle$. In this limit, it is therefore possible to achieve subnatural linewidth photons, that is, photons that are narrower than the natural spontaneous emission linewidth of $90 \mathrm{MHz}$ (corresponding lifetime $\left.\tau_{e} \approx 1.8 \mathrm{~ns}\right)$. When we measure the linewidth of the Raman photons in Fig. D.8B, we find that the linewidth is limited by the resolution of the FP cavity $180 \mathrm{MHz}$. When we use a different FP cavity with a resolution of $30 \mathrm{MHz}$, we find that the Raman linewidth is less than $30 \mathrm{MHz}$ (inset of Fig. D.8B), demonstrating subnatural-linewidth Raman photon emission. 


\section{D.7 Experimental procedure for entanglement gen- eration}

To generate entanglement between two SiV centers, we perform the same Raman tuning technique simultaneously on two spatially-separated $\mathrm{SiV}$ centers in a single diamond waveguide. The waveguide is again adiabatically coupled to a single-mode optical fiber as described in Sec. D.2.6. The collected photons are frequency filtered (see below) and then sent to a Hanbury Brown-Twiss setup to measure the photon autocorrelation function using the detectors and electronics described in Sec. D.1. The theoretical analysis of the system will be presented in Sec. D.8.

For the photon autocorrelation measurements performed when the two SiV centers are Raman-tuned onto resonance (Fig. 7.5B, red curve in Fig. 7.5D), the Raman fluorescence from both $\mathrm{SiV}$ centers passes through the FP cavity (FWHM: $150 \mathrm{MHz}$, FSR: $37 \mathrm{GHz}$, Transmission: 80\%), ensuring frequency indistinguishability. For the two SiVs used in the entanglement experiment, the transition frequencies $\nu_{e c}$ were detuned by about $1 \mathrm{GHz}$. The emitters were excited below saturation using two frequency stabilized lasers at single photon detunings $\Delta_{1}=1.3 \mathrm{GHz}$ for SiV1 and $\Delta_{2}=2.3 \mathrm{GHz}$ for $\mathrm{SiV} 2$, and the Raman emission frequencies and intensities were matched. During the course of the measurement, the Raman emission frequency for the $\mathrm{SiV}$ centers underwent slow spectral drifts of order $100 \mathrm{MHz}$ at the $\sim 10$ minute timescale that were compensated by the frequency of the excitation lasers. In other words, effects of slow spectral diffusion were mitigated using the Raman tuning technique. The Raman emission rates into the waveguide mode from each $\mathrm{SiV}$ were 
independently monitored to assure balanced emission. For these measurements, it is also important that the relative phase of the two driving lasers does not drift over the $\sim 10$ ns lifetime of the entangled state (see Sec. D.8.1 for a theoretical discussion). This requirement is fulfilled by the frequency stabilization scheme described in Sec. D.1. We experimentally verify the relative phase stability of the two driving fields by performing a time-domain interference measurement (Fig. D.9). For applications where it is necessary to achieve phase stability over longer timescales, the two excitation frequencies could be generated from a single laser using electrooptical modulation.

For the autocorrelation measurements performed in the distinguishable case (Fig. 7.5C, blue curve in Fig. 7.5D), the frequencies of the Raman photons from the two SiV centers are not tuned on resonance and differ by about $1 \mathrm{GHz}$. In this measurement, we split the fluorescence from the two $\mathrm{SiV}$ centers using a 50/50 beamsplitter and send each path to a separate FP cavity: FP1 (FWHM: $150 \mathrm{MHz}$, FSR: $37 \mathrm{GHz}$, Transmission: 80\%) is tuned to SiV1 Raman emission, FP2 (solid etalon with FWHM: $800 \mathrm{MHz}$, FSR: $20.3 \mathrm{GHz}$, Transmission: $80 \%$ ) is tuned to SiV2 Raman emission. The two paths are then recombined on a second 50/50 beamplitter and sent to two detectors to measure photon correlations. 

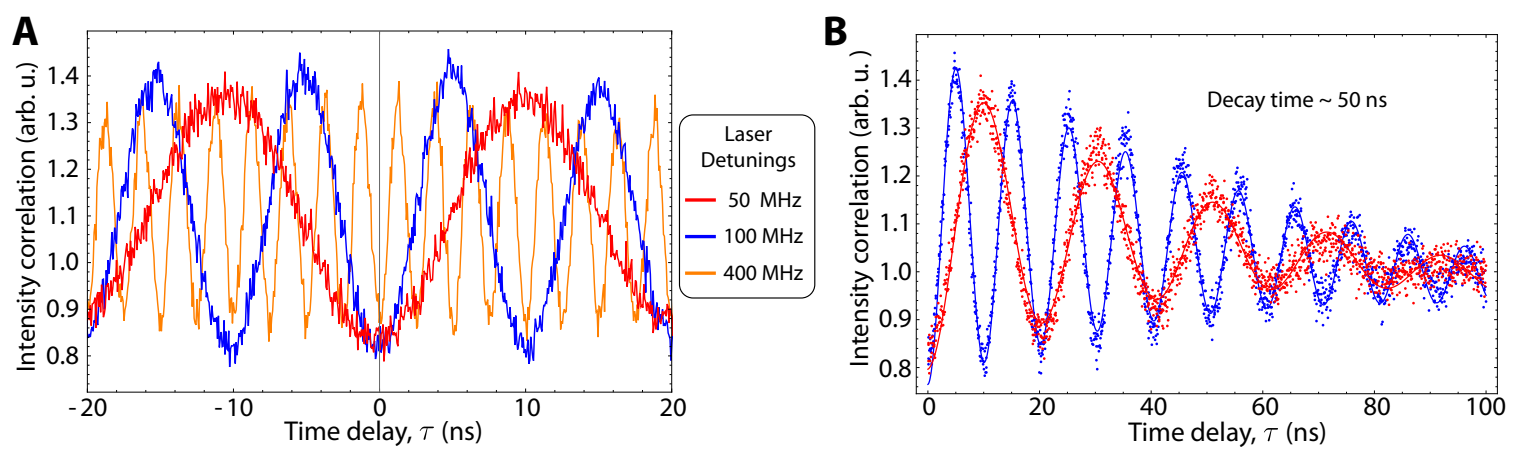

Figure D.9: Phase stability of the two Raman excitation lasers. The two lasers are combined on a beamsplitter and the intensity correlations are measured at the two output ports. (A) When the two lasers are detuned, intensity beats are observed. For the timescales considered in the entanglement experiment $(\tau<10 \mathrm{~ns}$, see Fig. 7.5D), the laser beats do not decay and the relative phase of the two lasers is stable. (B) The laser beats decay at a longer timescale of $\sim 50 \mathrm{~ns}$ that is determined by the relative linewidths of the two lasers.

\section{D.8 Model description for entanglement in a two-}

\section{SiV system}

In this Section, we analyze entanglement generation and verification for two $\mathrm{SiV}$ centers coupled to a waveguide. Specifically, we first use a simple model to describe the two-SiV entanglement generation process (Sec. D.8.1). We next extend this model in Sec. D.8.2 to include dissipation and show that it can be used to describe the

observations reported in the main text. We next present the analyses showing how the photon correlation measurements can be used to verify entanglement generation. Specifically, in Sec. D.8.3, we derive an expression for the concurrence of the two$\mathrm{SiV}$ system after emission of a single photon. We show that the concurrence is positive, demonstrating the presence of entanglement after a single photon emission. In Sec. D.8.3, we use a more specific model for our system to estimate the fidelity of 
the entangled state $|B\rangle$ conditioned on detection of two photons within a short time delay. The resulting fidelity also demonstrates creation of an entangled state.

\section{D.8.1 Simple model}

The process of entanglement generation can be understood through a simple model of the system. The two SiVs in the waveguide can be modeled as two three-level systems in a lossy cavity as in Sec. D.5. In a proper rotating frame, the Hamilton of the system is

$$
\begin{aligned}
\hat{H}= & \Delta_{1}|e\rangle_{1}\left\langle e\left|+\Delta_{2}\right| e\right\rangle_{2}\langle e|+\left(\Omega_{1}|e\rangle_{1}\left\langle u\left|+\Omega_{2}\right| e\right\rangle_{2}\langle u|+\text { h.c. }\right) \\
& +\left(g_{1}|e\rangle_{1}\left\langle c\left|\hat{a}+g_{2}\right| e\right\rangle_{2}\langle c| \hat{a}+\text { h.c. }\right)
\end{aligned}
$$

where we have assumed that both Raman transitions are on resonance and defined $\Delta_{i}=\omega_{e i}-\omega_{L i}$ where $\omega_{e i}$ is the frequency of level $|e\rangle_{i}$ and $\omega_{L i}$ is the frequency of the laser associated with the coupling $\Omega_{i}$, which we assume to be real. The photonic operator $\hat{a}$ describes the single mode field that both emitters couple to. Since we are operating in the far detuned regime, we can adiabatically eliminate the excited states, which results in an effective Hamiltonian

$$
\hat{H}_{\mathrm{eff}} \approx-\tilde{g}_{1} \hat{\sigma}_{u c}^{(1)} \hat{a}-\tilde{g}_{2} \hat{\sigma}_{u c}^{(2)} \hat{a}+h . c
$$

where $\tilde{g}_{i} \approx \frac{\Omega_{i} g_{i}}{\Delta_{i}}$ and we have defined $\hat{\sigma}_{u c}^{(i)}=|u\rangle_{i}\langle c|$. We have neglected any shifts of the ground states due to laser and cavity couplings since we are far detuned.

To understand entanglement generation, we consider the initial state of the SiVs $|u\rangle_{1}|u\rangle_{2}$ since this is the only state that can emit two Raman photons. The evolution 
of this initial state under the above Hamiltonian is

$$
U(\epsilon)|u\rangle_{1}|u\rangle_{2}|0\rangle \approx|u\rangle_{1}|u\rangle_{2}|0\rangle+i \epsilon\left(\tilde{g}_{1}|c\rangle_{1}|u\rangle_{2}+\tilde{g}_{2}|u\rangle_{1}|c\rangle_{2}\right)|1\rangle,
$$

where $U(t)=e^{-i \hat{H} t},|0\rangle(|1\rangle)$ is the vacuum (single photon) state of the waveguide mode and we have expanded the time evolution assuming that we are looking at a time $\epsilon \ll 1 / \tilde{g}_{1,2}$. In the experiment, the strength of the lasers is tuned such that $\left|\tilde{g}_{1}\right|=\left|\tilde{g}_{2}\right|=\tilde{g}$ and we write $g_{2}=g_{1} e^{i \phi}$ where $\phi$ results from the propagation phase between the emitters in the waveguide and the relative phase of the two driving lasers. As described above, the two driving lasers are frequency stabilized such that the phase $\phi$ is constant over timescales much longer than any other system dynamics. Under these conditions, we have

$$
U(\epsilon)|u\rangle_{1}|u\rangle_{2}|0\rangle \approx|u\rangle_{1}|u\rangle_{2}|0\rangle+i \epsilon \tilde{g}\left(|c\rangle_{1}|u\rangle_{2}+e^{i \phi}|u\rangle_{1}|c\rangle_{2}\right)|1\rangle .
$$

The emission of the first photon thus prepares the $\mathrm{SiVs}$ in the entangled state

$$
|B\rangle=\left(|c\rangle_{1}|u\rangle_{2}+e^{i \phi}|u\rangle_{1}|c\rangle_{2}\right) / \sqrt{2} .
$$

This state $|B\rangle$ is a superradiant state, which emits photons at a rate which is twice that of a single emitter in state $|u\rangle$. This can be seen from the evolution of this state under the Hamiltonian in Eq. (D.18):

$$
U(\epsilon)|B\rangle|0\rangle \approx|B\rangle|0\rangle-\sqrt{2} i \epsilon \tilde{g} e^{i \phi}|c\rangle_{1}|c\rangle_{2}|1\rangle .
$$

The $\sqrt{2}$ prefactor of $|c\rangle_{1}|c\rangle_{2}|1\rangle$ corresponds to a factor of 2 enhancement of the emission rate compared to that of a single emitter in state $|u\rangle$. It is this enhanced emission rate that results in the measured peak in the normalized photon correlation function $g^{(2)}$ at zero delay time in Fig. 7.5D. 


\section{D.8.2 Detailed model}

We next present a more detailed model of the waveguide experiment including various dissipation channels in order to model the time-dependent $g^{(2)}$-correlation functions. We describe the unitary dynamics using Eq. (D.17) and the dissipation in the system using the Lindblad operators defined in Eq. (D.2) for each atom. The

only exception is that we now define $\hat{L}_{5, i}=\sqrt{\gamma_{d}}|u\rangle_{i}\langle u|$ because we scatter Raman photons at a large detuning $\left(\Delta_{i} \gg \gamma_{u}\right)$ where the contribution from excited state dephasing can be neglected and ground state dephasing dominates. Furthermore, we assume that the two atoms have equal decay rates. Since $\Delta_{i} \gg \Omega_{i}, g_{i}$, we can again adiabatically eliminate the excited states, which results in the effective Hamiltonian in Eq. (D.18). The effective Lindblad operators are $\hat{L}_{3, i}$ through $\hat{L}_{6, i}$ and

$$
\begin{aligned}
& \hat{L}_{1, i}^{\mathrm{eff}} \approx \frac{\sqrt{\gamma_{c}}}{\Delta_{i}}\left(\Omega_{i}|c\rangle_{i}\left\langle u\left|+g_{i}\right| c\right\rangle_{i}\langle c| \hat{a}\right) \\
& \hat{L}_{2, i}^{\mathrm{eff}} \approx \frac{\sqrt{\gamma_{u}}}{\Delta_{i}}\left(\Omega_{i}|u\rangle_{i}\left\langle u\left|+g_{i}\right| u\right\rangle_{i}\langle c| \hat{a}\right) .
\end{aligned}
$$

Since we operate in the weak-driving regime, we can truncate the Hilbert space assuming that at most two photons are present in the system. In this case, the system can be described in a basis of the states

$$
\begin{aligned}
& \{|u u\rangle|0\rangle,|u c\rangle|0\rangle,|c u\rangle|0\rangle,|c c\rangle|0\rangle,|u u\rangle|1\rangle,|u c\rangle|1\rangle, \\
& |c u\rangle|1\rangle,|c c\rangle|1\rangle,|u u\rangle|2\rangle,|u c\rangle|2\rangle,|c u\rangle|2\rangle,|c c\rangle|2\rangle\},
\end{aligned}
$$

which we label $\{|1\rangle, \ldots,|12\rangle\}$. The notation is such that $|u c\rangle|1\rangle$ denotes atomic state $|u\rangle_{1}|c\rangle_{2}$ and one photon in mode $\hat{a}$. In this basis, we can solve the master equation for the system by adiabatically eliminating all states containing cavity photons since the cavity mode models, in this experiment a waveguide, and therefore has extremely 
fast decay. We also assume that the dynamics of the ground states $\{|1\rangle, \ldots,|4\rangle\}$ are governed solely by the dissipative couplings between them (similar to the procedure in Sec. D.5.3) since we are in the weak driving regime and that $\left|g_{i}\right|^{2} /\left(\kappa \gamma_{u}\right) \ll 1$.

Under these conditions, we find that the photon intensity correlation function can be expressed as

$$
g^{(2)}(\tau) \approx \frac{\left(\alpha_{1}+\alpha_{2}\right) \rho_{1,1}(\tau)+\alpha_{1} \rho_{2,2}(\tau)+\alpha_{2} \rho_{3,3}(\tau)+2 \alpha_{3} \rho_{2,3}(\tau)}{\left(\left(\alpha_{1}+\alpha_{2}\right)\left(\rho_{1,1}^{(s)}+\rho_{2,2}^{(s)}\right)\right)^{2}},
$$

where $\rho_{i, j}=\langle i|\rho| j\rangle$ and $\rho^{(s)}$ denotes the steady state density matrix of the system and $\rho=\hat{a} \rho^{(s)} \hat{a}^{\dagger}$. The matrix elements and constants are defined in Sec. D.9.3. The peak in the $g^{(2)}$ function at zero time-delay originates from the coherence $\alpha_{3} \rho_{2,3}(\tau)$ between the two ground states. This is consistent with the simple model description in Sec. D.8.1: If the laser strengths are tuned such that $\left|\frac{\Omega_{1}^{*} g_{1}}{\Delta_{1}}\right|=\left|\frac{\Omega_{2}^{*} g_{2}}{\Delta_{2}}\right|$ and $g_{2}=g_{1} e^{i \phi}$ then $\rho_{1,1}(0)=0$ and $\alpha_{1} \rho_{2,2}(0)=\alpha_{2} \rho_{3,3}(0)=\alpha_{3} \rho_{2,3}(0)$, which means that the peak is a result of the enhanced emission rate of the entangled state $|B\rangle$. The coherence $\rho_{2,3}(\tau)$ decays with a rate $\Gamma \approx \gamma_{c u}+\gamma_{u c}+\gamma_{d}$, which determines the width of the peak. We note that under the continuous driving used in our experiment, the optical pumping rate out of state $|u\rangle$ determines the lower limit on this decoherence rate.

Eq. (D.26) corresponds to the ideal limit where no noise photons, e.g. originating from scattering of laser light, are detected. The effect of noise photons changes the correlation function into

$$
g_{\text {noise }}^{(2)}(\tau) \approx \frac{g^{(2)}(\tau)+\left(2+p_{n}\right) p_{n}}{\left(1+p_{n}\right)^{2}}
$$

where $p_{n}$ is the relative rate (odds) of detecting noise photons compared to Raman photons and we have assumed that the noise photons are completely classical and 


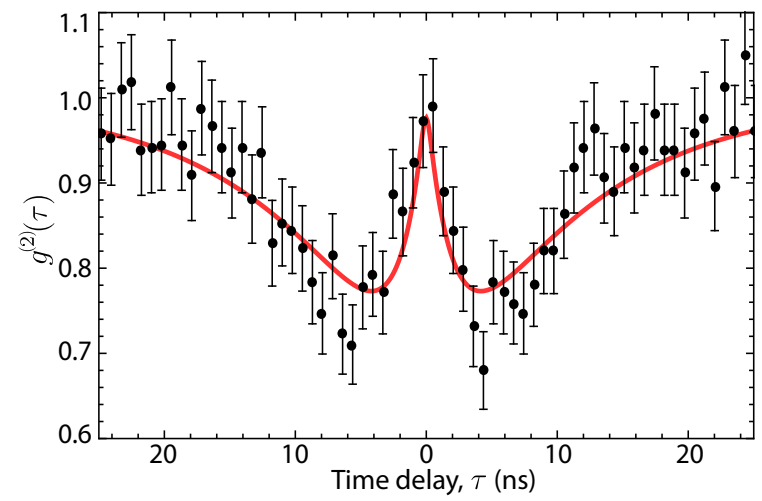

Figure D.10: Comparison of model (solid red curve) to data (black points) using the following parameters (defined in Eq. (D.2)): $\left\{\Delta_{1}, \Delta_{2}, \gamma_{c u}, \gamma_{u c}, \gamma_{u u}, \Omega_{1}, \Omega_{2}\right\}=$ $\{22,14,0.10,0.06,0.66,0.23,0.15\} \times \gamma_{0}$ with $\gamma_{0}=2 \pi \times 94 \mathrm{MHz}$. The model has been convolved with a Gaussian to account for the finite detector jitter present in the experiment. Of these parameters, $\Delta_{1}, \Delta_{2}$ and the odds of detecting a noise photon $p_{n}=0.09$ are fixed based on single-photon detunings and single $\mathrm{SiV} g^{(2)}$ measurements; the other parameters are estimated by fitting the data to the model under conservative physical constraints. The data correspond to the red curve in Fig. 5D in the main text.

uncorrelated. The results of this model are in good agreement with the measured data as shown in Fig. D.10. The solid curves in Fig. 7.5D are a simple phenomenological model assuming exponential decay with a single timescale for the distinguishable and single-SiV cases and two timescales for the indistinguishable case. All models are convolved with the (independently measured) temporal response of the cavity and photon detectors.

\section{D.8.3 Entanglement analysis}

We verify entanglement generation through the two-photon correlation function of the emitted field from the SiVs. The SiVs are excited by weak continuous-wave lasers and the corresponding emitted field is a stationary field such that the photon 
correlation function of the field can be written as

$$
\begin{aligned}
g^{(2)}(\tau) & =\frac{\operatorname{tr} \hat{A}^{\dagger} \hat{A}^{\dagger}(\tau) \hat{A}(\tau) \hat{A} \rho}{\operatorname{tr} \hat{A} \hat{A} \rho^{2}}=\frac{\operatorname{tr} \hat{A}^{\dagger} \hat{A} e^{\mathcal{L} \tau}\left[\hat{A} \rho \hat{A}^{\dagger}\right]}{\operatorname{tr} \hat{A}^{\dagger} \hat{A} \rho^{2}} \\
& =\frac{\operatorname{tr} \hat{A}^{\dagger} \hat{A} \tilde{\rho}(\tau)}{\operatorname{tr} \hat{A}^{\dagger} \hat{A} \rho},
\end{aligned}
$$

where $\hat{A}=\hat{A}(0)$ is the annihilation operator of the field and $\rho$ is the density matrix describing the stationary field, i.e. the steady state density matrix of the system. We have defined $\tilde{\rho}(\tau)$ as the conditional density matrix at time $\tau$ with initial condition $\tilde{\rho}(0)=\frac{1}{\operatorname{tr} \hat{A} \dagger \hat{A} \rho} \hat{A} \rho \hat{A}^{\dagger}$ and time evolution described by some Lindblad super-operator $\mathcal{L} \tau$. $\quad \tilde{\rho}(\tau)$ thus describes the state of the system at time $\tau$ conditioned on having emitted a photon at time $\tau=0$. Note that in the experiment, the photon is detected with a fixed time delay after being emitted into into the waveguide. However, the detection still heralds that the photon was emitted into the waveguide at the earlier time. The photon correlation function $g^{(2)}(\tau)$ thus directly probes the ratio between photon emission from the conditionally prepared state after emission of a photon into the waveguide and the stationary state $\rho$.

\section{Concurrence}

The degree of entanglement of an arbitrary two qubit state can be quantified by the concurrence of the corresponding density matrix, $\mathcal{C}(\rho)$ [235]. All separable states have $\mathcal{C}(\rho)=0$ while a maximally entangled state has $\mathcal{C}(\rho)=1$. Following the procedure of Ref. [236], we derive a lower bound on the concurrence of the state of the two SiVs conditioned on a photon emission into the waveguide. We show that the concurrence is positive, demonstrating the presence of entanglement after a single photon emission. 
As a general model for the $\mathrm{SiVs}$, we describe them as qubits with states $|c\rangle$ and $|u\rangle$ where a photon can be emitted from state $|u\rangle$ with some fixed probability while $|c\rangle$ is dark. Let $p_{u, i}^{(0)}$ be the probability of the $i^{\text {th }} \mathrm{SiV}$ to be in state $|u\rangle$ in the steady state. According to Eq. (D.29), the corresponding single SiV photon correlation function can then be written as

$$
g_{i}^{(2)}(\tau)=\frac{p_{u, i}(\tau)}{p_{u, i}^{(0)}},
$$

where $p_{u, i}(\tau)$ is the probability of the $\mathrm{SiV}$ to be in state $|u\rangle$ time $\tau$ after the emission of the first photon. If both SiVs are continuously excited, we can write the density matrix following the emission of a photon as

$$
\tilde{\rho}=\left(\begin{array}{cccc}
p_{c c} & 0 & 0 & 0 \\
0 & p_{u c} & d & 0 \\
0 & d^{*} & p_{c u} & 0 \\
0 & 0 & 0 & p_{u u}
\end{array}\right),
$$

in the basis $\{|c c\rangle,|c u\rangle,|u c\rangle,|u u\rangle\}$. Here, $p_{c u}$ is the (time-dependent) probability of the first $\mathrm{SiV}$ being in state $|c\rangle$ and the second $\mathrm{SiV}$ being in state $|u\rangle$, and so on. Note that we have assumed there is no coherence in the system except between states $|c u\rangle$ and $|u c\rangle$. This assumption gives a lower bound on the actual amount of entanglement in the system since any coherences could be removed with local operations on the SiVs and classical communication, which can never increase the amount of entanglement as described in Ref. [236]. The concurrence of the conditional density matrix is [236]

$$
\mathcal{C}(\tilde{\rho})=\max \left(2|d|-2 \sqrt{p_{c c} p_{u u}}, 0\right) .
$$

We assume that the two SiVs are completely uncorrelated in the steady state before a photon emission and that we are detecting a mode $\hat{A} \sim \hat{S}_{1}+e^{i \phi} \hat{S}_{2}$, where $\hat{S}_{i}$ is the 
operator associated with emission of a photon from the $i^{\text {th }} \mathrm{SiV}$. The $g^{(2)}$ function can then be written as

$$
g^{(2)}(\tau)=\frac{p_{c u}+p_{u c}+2|d| \cos (\theta-\phi)+2 p_{u u}}{p_{u, 1}^{(0)}+p_{u, 2}^{(0)}},
$$

where we have written the coherences as $d=|d| e^{i \theta}$. From this, we obtain a lower bound on the coherences

$$
2|d| \geq\left|g_{\text {indist }}^{(2)}(\tau)\left(p_{u, 1}^{(0)}+p_{u, 2}^{(0)}\right)-1-p_{u u}+p_{c c}\right|,
$$

where we have used that $p_{u c}+p_{c u}=1-p_{c c}-p_{u u}$. The diagonal elements $p_{u u}$ and $p_{c c}$ can be obtained from the single-SiV $g_{i}^{(2)}$ functions as

$$
p_{u u}=\frac{p_{u, 1}^{(0)} p_{u, 2}^{(0)}}{p_{u, 1}^{(0)}+p_{u, 2}^{(0)}}\left(p_{u, 1}(\tau)+p_{u, 2}(\tau)\right)=\frac{p_{u, 1}^{(0)} p_{u, 2}^{(0)}}{p_{u, 1}^{(0)}+p_{u, 2}^{(0)}}\left(g_{1}^{(2)}(\tau) p_{u, 1}^{(0)}+g_{2}^{(2)}(\tau) p_{u, 2}^{(0)}\right),
$$

and

$$
\begin{aligned}
p_{c c} & =\frac{1}{p_{u, 1}^{(0)}+p_{u, 2}^{(0)}}\left(p_{c, 1}(\tau) p_{c, 2}^{(0)} p_{u, 1}^{(0)}+p_{c, 2}(\tau) p_{c, 1}^{(0)} p_{u, 2}^{(0)}\right) \\
& \left.=\frac{1}{p_{u, 1}^{(0)}+p_{u, 2}^{(0)}}\left(\left(1-g_{1}^{(2)}(\tau) p_{u, 1}^{(0)}\right)\left(1-p_{u, 2}^{(0)}\right) p_{u, 1}^{(0)}+\left(1-g_{2}^{(2)}(\tau) p_{u, 2}^{(0)}\right)\left(1-p_{u, 1}^{(0)}\right) p_{u, 2}^{(\phi)}\right) \cdot 37\right)
\end{aligned}
$$

where we have used that $p_{c, i}^{(0)}=1-p_{u, i}^{(0)}$ and $p_{c, i}=1-p_{u, i}$. Combining Eqs. (D.34)(D.36) with Eq. (D.32) gives a lower bound on the concurrence. For the experiment, the photon detection rates from the $\mathrm{SiVs}$ were balanced such that $p_{u, 1}^{(0)}=p_{u, 2}^{(0)}=p_{u}^{(0)}$ and the lower bound becomes

$$
\begin{aligned}
\mathcal{C}(\tilde{\rho}) \geq & \max \left(0,\left|2 g_{\text {indist }}^{(2)}(\tau) p_{u}^{(0)}-\frac{1}{2}\left(2+g_{1}^{(2)}(\tau)+g_{2}^{(2)}(\tau)\right) p_{u}^{(0)}\right|\right. \\
& \left.-p_{u}^{(0)} \sqrt{\left(g_{1}^{(2)}(\tau)+g_{2}^{(2)}(\tau)\right)\left(2-\left(g_{1}^{(2)}(\tau)+g_{2}^{(2)}(\tau)\right) p_{u}^{(0)}\right)\left(1-p_{u}^{(0)}\right)}\right) \text { D. }
\end{aligned}
$$


We estimate the concurrence generated in our experiment by directly evaluating Eq. (D.38) using experimentally measured values of $g^{(2)}(\tau)$ at short time delays $\tau \sim 0$ (Fig. 7.5). We estimate the error assuming the photon count rates follow a Poisson distribution. (This assumption has been verified via similar measurements on the same apparatus). By taking the thermal distribution at $4 \mathrm{~K}$ and measured saturation associated with weak optical pumping during the measurement into account, we obtain a

lower bound of $p_{u}^{(0)} \geq 0.34$ for each $\mathrm{SiV}$ in steady state. From fits of the autocorrelation functions shown in Fig. 7.5D, we obtain the concurrence $\mathcal{C}(\tau=0)>0.090(0.024)$ demonstrating entanglement generation in the system. Around $\tau=0$ the concurrence is fairly insensitive to this window size and is positive for window sizes up to $8 \mathrm{~ns}$. All uncertainties given in parentheses here and throughout the manuscript are the one-standard-deviation level. Note that the extracted value of concurrence is limited by imperfect initial state preparation of the SiV centers. This can be circumvented by either working with the pulsed excitation or via post-selection as discussed in the following section.

\section{Conditional fidelity estimate}

The lower bound on the concurrence extracted above verifies that entanglement is created by emission of the first photon into the waveguide. In order to relate this entanglement to the theoretical model presented previously, we now estimate the fidelity of the conditional state with state $|B\rangle$ given that a second photon is detected at a time $\epsilon$ after the first. Here, $\epsilon$ is much shorter than the decoherence rate of the ground states, which ensures that any dynamics of the conditional state can be 
neglected and we therefore post-select on events where the initial state of the SiVs was $|u u\rangle$ to good approximation.

While the measurement of the correlation function of a single $\mathrm{SiV}$ individually shows a strong anti-bunching at $\tau=0$ (orange curve in Fig. 7.5D), the correlation functions do not completely vanish as expected for an ideal single photon source. What is detected is therefore not only the field from the Raman transition of the $\mathrm{SiV}$ but also noise photons originating primarily from the scattering of frequencydistinguishable laser light. From the single-emitter $g_{i}^{(2)}$ functions, we can estimate the ratio, $p_{n, i}$ between the detection rate of noise photons and Raman photons assuming that the noise photons are classical and uncorrelated. From Eq. (D.27), we find that

$$
g_{i}^{(2)}(0)=\frac{p_{n, i}\left(2+p_{n, i}\right)}{\left(1+p_{n, i}\right)^{2}}
$$

which for the measured single emitter $g^{(2)}$ functions of $g_{1}^{(2)}(0)=g_{2}^{(2)}(0)=0.16(3)$ gives $p_{n, 1}=p_{n, 2}=p_{n}=0.09(2)$.

To calculate the $g^{(2)}$ function when both emitters are excited, we assume that the emitters are completely uncorrelated in the steady state. Consequently, the rate of Raman photons $\gamma_{R}$ is simply the sum of the Raman photon rates from the single emitters in the steady state. Furthermore, the rate of noise photons $\gamma_{\text {noise }}$ is also assumed to be the sum of the noise photon rates for the single emitters. In the experiments, the photon detection rate from the two emitters was balanced, which means that the ratio between the detection rate of Raman photons and noise photons is still $p_{n}$. The total rate of photons from the steady state can thus be written as $\gamma_{\text {total }}=\gamma_{R}+\gamma_{\text {noise }}=2 \gamma_{r} p_{u}^{(0)}\left(1+p_{n}\right)$, where we have written the single emitter Raman photon rate as $\gamma_{r} p_{u}^{(0)}$ where $p_{u}^{(0)}$ is the steady state probability of an emitter to be in 
state $|u\rangle$.

The conditional density matrix upon emission of the first photon can be parameterized as

$$
\tilde{\rho}=\frac{1}{1+p_{n}}\left(p_{u}^{(0)} \tilde{F}|B\rangle\langle B|+\left(1-p_{u}^{(0)} \tilde{F}\right) \rho_{d}\right)+\frac{p_{n}}{1+p_{n}} \rho,
$$

where $\rho$ is the steady state density matrix of the $\mathrm{SiVs}$ and $\rho_{d}$ describes all states that cannot emit Raman photons, i.e. $|c c\rangle$ and $|D\rangle=\frac{1}{\sqrt{2}}\left(|u c\rangle-e^{i \phi}|c u\rangle\right)$. The first term proportional to $\frac{1}{1+p_{n}}$ can be viewed as the conditional density matrix if the first detected photon was a Raman photon while the second term proportional to $\frac{p_{n}}{1+p_{n}}$ is where the first detected photon was a noise photon. This conditional density matrix results in a $g^{(2)}$ function

$$
g^{(2)}(0)=\frac{\tilde{F}+p_{n}\left(2+p_{n}\right)}{\left(1+p_{n}\right)^{2}} .
$$

$\tilde{F}$ can thus be extracted from the the measured value of $g^{(2)}(0)$ in Fig. 7.5 D. $\tilde{F}$ can be viewed as the fidelity with state $|B\rangle$ if the two SiVs were initially in state $|u u\rangle$.

In the $g^{(2)}$ measurements, we postselect for experimental runs that result in a two-photon coincidence. For these runs, the overlap with state $|B\rangle$ after the emission of the first photon is then given by $F=p_{u u}^{(c)} \tilde{F}$, where $p_{u u}^{(c)}$ is the probability of the initial state being $|u u\rangle$ given that we detected the second photon $\epsilon$ after the first photon. This probability can be bounded from below by the relative rate of detecting two Raman photons compared to the total rate of detecting two photons. From Eq. (D.40), we find that this is

$$
p_{u u}^{(c)} \geq \frac{\frac{1}{1+p_{n}} 2 \tilde{F} p_{u}^{(0)} \gamma_{s}}{\frac{1}{1+p_{n}} 2 \tilde{F} p_{u}^{(0)} \gamma_{s}+2 p_{n} p_{u}^{(0)} \gamma_{s}+\frac{p_{n}}{1+p_{n}} 2 \gamma_{s} p_{u}^{(0)}}=\frac{\tilde{F}}{\tilde{F}+\left(2+p_{n}\right) p_{n}} .
$$


Consequently, we find a lower bound on the fidelity of the conditional state

$$
F \geq \frac{\left(g^{(2)}(0)\left(1+p_{n}\right)^{2}-p_{n}\left(2+p_{n}\right)\right)^{2}}{g^{(2)}(0)\left(1+p_{n}\right)^{2}} .
$$

From the measured value of $g^{(2)}(0)=0.98(5)$, we obtain $F \geq 82(7) \%$.

\section{D.9 Analytical expressions for density matrix ele- ments}

\section{D.9.1 Steady-state density matrix expressions}

The analytical expression for the matrix elements $\rho_{i, j}^{(s)}=\left\langle i\left|\rho^{(s)}\right| j\right\rangle$ in Sec. D.5.2 are given by:

$$
\begin{aligned}
\rho_{1,1}^{(s)} \approx & \frac{1}{\mathcal{A}+\mathcal{B} / \mathcal{C}} \\
\rho_{1,2}^{(s)} \approx & \frac{-4 \xi g \rho_{1,1}^{(s)}}{\mathcal{C}} \\
& \times\left(16 \xi^{2} g^{2}-\kappa\left(\left(4 \Delta_{e}^{2}+\kappa^{2}\right)\left(\gamma_{c}+\gamma_{u}\right)+4 g^{2}\left(\kappa+\gamma_{c}+\gamma_{u}\right)\right)\right)\left(4 g^{2}+\kappa\left(2 i \Delta_{e}+\Gamma\right)(\mathrm{D} .45)\right. \\
\rho_{2,2}^{(s)} \approx & \frac{16 \xi^{2} g^{2} \kappa(\kappa+\Gamma)\left(4 g^{2}+\kappa \Gamma\right) \rho_{1,1}^{(s)}}{\mathcal{C}}, \\
\rho_{3,3}^{(s)} \approx & \frac{\gamma_{u} \rho_{2,2}^{(s)}+\gamma_{u c} \rho_{1,1}^{(s)}}{\gamma_{c u}} \\
\rho_{4,4}^{(s)} \approx & \frac{4 \xi^{2} \rho_{1,1}^{(s)}}{\mathcal{C}}\left(16 \Delta_{e}^{4} \kappa\left(\gamma_{c}+\gamma_{u}\right)+\left(4 g^{2}+\kappa \Gamma\right)\left(16 \xi^{2} g^{2}+\kappa\left(4 g^{2}\left(\gamma_{d}+\gamma_{u c}\right)+\kappa\left(\gamma_{c}+\gamma_{u}\right) \Gamma\right)\right)\right. \\
& \left.+4 \Delta_{e}^{2} \kappa\left(\gamma_{c}+\gamma_{u}\right)\left(\kappa^{2}+\Gamma^{2}\right)+4 \Delta_{e}^{2}\left(-16 \xi^{2} g^{2}+4 g^{2}\left(\kappa^{2}+(\kappa+\Gamma)\left(\gamma_{c}+\gamma_{u}\right)\right)\right)\right)(\mathrm{D} .48)
\end{aligned}
$$




$$
\begin{aligned}
\rho_{5,5}^{(s)} \approx & \frac{4 \xi^{2}}{\kappa^{2}} \rho_{2,2}^{(s)} \\
\rho_{6,6}^{(s)} \approx & \frac{4 \xi^{2}}{\kappa^{2}} \rho_{3,3}^{(s)} \\
\rho_{7,7}^{(s)} \approx & \frac{4 \xi^{2} \rho_{4,4}^{(s)}+4 g^{2} \rho_{5,5}^{(s)}}{3 \kappa^{2}}-\frac{8}{27 \kappa^{4}}\left(-9 \xi^{4} \rho_{1,1}^{(s)}+45 \xi^{3} g\left(\rho_{1,2}^{(s)}+\rho_{2,1}^{(s)}\right)\right. \\
& \left.+12 \xi^{2} g^{2}\left(-9 \rho_{2,2}^{(s)}+7 \rho_{4,4}^{(s)}\right)+2 g^{2}\left(\Delta_{e}^{2}+3 g^{2}\right) \rho_{5,5}^{(s)}\right)+\frac{392 i \Delta_{e} \xi^{3} g}{9 \kappa^{5}}\left(\rho_{1,2}^{(s)}-k(\mathrm{D} 2 \mathrm{~s}) .1 .51\right) \\
\rho_{8,8}^{(s)} \approx & \frac{6 \xi^{2}}{3 \kappa^{2}} \rho_{6,6}^{(s)},
\end{aligned}
$$

where we have defined

$$
\begin{aligned}
\mathcal{A}= & \frac{\gamma_{c u}+\gamma_{u c}}{\gamma_{c u}}+\left(\frac{8 \xi^{4}+4 \xi^{2} \kappa^{2}}{\kappa^{4}}\right) \frac{\gamma_{u c}}{\gamma_{c u}} \\
\mathcal{B}= & \frac{16 g^{2} \xi^{2}}{3 \kappa^{3} \gamma_{c u}}\left(24 \xi^{4} \gamma_{u}+3 \kappa^{4}\left(\gamma_{c u}+\gamma_{u}\right)+4 \xi^{2}\left(4 g^{2} \gamma_{c u}+3 \kappa^{2}\left(\gamma_{c u}+\gamma_{u}\right)\right)\right)(\kappa+\Gamma)\left(4 g^{2}+\kappa \Gamma\right) \\
& +\left(4 \xi^{2}+\frac{16 \xi^{4}}{3 \kappa^{2}}\right)\left(16 \Delta_{e}^{4} \kappa\left(\gamma_{c}+\gamma_{u}\right)+\left(4 g^{2}+\kappa \Gamma\right)\left(16 \xi^{2} g^{2}+\kappa\left(4 g^{2}\left(\gamma_{d}+\gamma_{u c}\right)+\kappa\left(\gamma_{c}+\gamma_{u}\right) \Gamma\right)\right)\right. \\
& \left.+4 \Delta_{e}^{2}\left(-16 \xi^{2} g^{2}+4 g^{2}\left(\kappa^{2}+(\kappa+\Gamma)\left(\gamma_{c}+\gamma_{u}\right)\right)+\kappa\left(\gamma_{u}+\gamma_{c}\right)\left(\kappa^{2}+\Gamma^{2}\right)\right)\right) \\
\mathcal{C}= & -16 \xi^{2} g^{2}\left(4 \Delta_{e}^{2} \kappa^{2}+\left(4 g^{2}-\kappa^{2}\right)\left(4 g^{2}+\kappa \Gamma\right)\right) \\
& +\kappa\left(\left(4 \Delta_{e}^{2}+\kappa^{2}\right)\left(\gamma_{c}+\gamma_{u}\right)+4 g^{2}\left(\kappa+\gamma_{c}+\gamma_{u}\right)\right)\left(4 \Delta_{e}^{2} \kappa^{2}+\left(4 g^{2}+\kappa \Gamma\right)^{2}\right) .
\end{aligned}
$$

\section{D.9.2 Coefficients in the expressions for the time-dependent density matrix elements}

Here, we give explicit expressions for the coefficients $a_{i}, b_{i}, \mathcal{A}_{i}, \mathcal{B}_{i}$ and $\mathcal{C}_{i}$ in the time-dependent density matrix elements described in Sec. D.5.3: 


$$
\begin{aligned}
\mathcal{A}_{1}=-\frac{a_{2}}{a_{1}+i \Delta_{e}}, & \mathcal{C}_{1}=\frac{1}{\kappa^{2}+4 g^{2}}\left(4 \xi^{2} \alpha-4 \xi g\left(\mathcal{A}_{1}+\mathcal{A}_{1}^{*}\right)+4 g^{2} \mathcal{B}_{1}\right), \\
\mathcal{A}_{2}=-\frac{a_{3}}{a_{1}+i \Delta_{e}+\tilde{\gamma}}, & \mathcal{C}_{2}=\frac{1}{\kappa^{2}+4 g^{2}}\left(4 \xi^{2} \beta-4 \xi g\left(\mathcal{A}_{2}+\mathcal{A}_{2}^{*}\right)+4 g^{2} \mathcal{B}_{2}\right), \\
\mathcal{A}_{3}=\left(-\mathcal{A}_{1}-\mathcal{A}_{2}+\rho_{1,2}(0)\right) e^{i \Delta_{e} t}, & \mathcal{C}_{3}=\frac{1}{\kappa^{2}+4 g^{2}}\left(-4 \xi g\left(\mathcal{A}_{3}+\mathcal{A}_{3}^{*}\right)+4 g^{2} \mathcal{B}_{3}\right) \\
\mathcal{B}_{1}=\frac{2 a_{1} a_{2} b_{2}}{b_{1}\left(a_{1}^{2}+\Delta_{e}^{2}\right)}-\frac{b_{3}}{b_{1}}, & \\
\mathcal{B}_{2}=\frac{-\left(a_{1}^{2}+\Delta_{e}^{2}\right) b_{4}+a_{1} a_{3} b_{2}}{\left(b_{1}+\tilde{\gamma}\right)\left(\Delta_{e}^{2}+\left(a_{1}+\tilde{\gamma}\right)^{2}\right)} & \mathcal{B}_{4}=-\left(\mathcal{B}_{1}+\mathcal{B}_{2}\right)+\rho_{2,2}(0) \\
-\frac{\left(b_{4}\left(2 a_{1}+\tilde{\gamma}\right)-2 a_{3} b_{2}\right)(\tilde{\gamma})}{\left(b_{1}+\tilde{\gamma}\right)\left(\Delta_{e}^{2}+\left(a_{1}+\tilde{\gamma}\right)^{2}\right)}, & -2 \Re\left[\frac{4 g^{2}}{\kappa_{1}+i \Delta_{e}-b_{1}} \mathcal{A}_{3} e^{-i \Delta_{e} t}\right], \\
\mathcal{B}_{3}=2 \Re\left[\frac{b_{2}}{a_{1}+i \Delta_{e}-b_{1}} \mathcal{A}_{3}\right], &
\end{aligned}
$$

and

$$
\begin{array}{rlrl}
b_{1} & =-\gamma_{c}-\gamma_{u}-\frac{4 g^{2} \kappa}{\kappa^{2}+4 g^{2}}, & & a_{1}=-\frac{\gamma_{u}+\gamma_{c}+\gamma_{d}+\gamma_{u c}}{2}-\frac{2 g^{2}}{\kappa}, \\
b_{2} & =\frac{2 \xi g}{\kappa}-\frac{16 g^{3} \xi}{\kappa\left(\kappa^{2}+4 g^{2}\right)}, & a_{2} & =\frac{2 g \xi}{\kappa} \alpha, \\
b_{3} & =\frac{16 \xi^{2} g^{2}}{\kappa\left(\kappa^{2}+4 g^{2}\right)} \alpha, & a_{3}=\frac{2 g \xi}{\kappa} \beta, \\
b_{4}=\frac{16 \xi^{2} g^{2}}{\kappa\left(\kappa^{2}+4 g^{2}\right)} \beta . & &
\end{array}
$$




\section{D.9.3 Coefficents and matrix elements for the two-SiV model}

The constants and matrix elements appearing in Sec. D.8.2 are defined as

$$
\begin{aligned}
\alpha_{1} & =\frac{4\left|g_{1}\right|^{2}\left|\Omega_{1}\right|^{2}}{\Delta_{1}^{2} \kappa^{2}}, & \alpha_{2} & =\frac{4\left|g_{2}\right|^{2}\left|\Omega_{2}\right|^{2}}{\Delta_{2}^{2} \kappa^{2}}, \\
\alpha_{3} & =\frac{4 g_{1}^{*} g_{2} \Omega_{2}^{*} \Omega_{1}}{\Delta_{1} \Delta_{2} \kappa^{2}}, & \rho_{1,1}^{(s)} & \approx \frac{\gamma_{u c}^{2}}{\left(\gamma_{u c}+\gamma_{c u}\right)^{2}} \\
\rho_{2,2}^{(s)} & \approx \frac{\gamma_{u c} \gamma_{c u}}{\left(\gamma_{u c}+\gamma_{c u}\right)^{2}}, & \rho_{4,4}^{(s)} & \approx \frac{\gamma_{c u}^{2}}{\left(\gamma_{u c}+\gamma_{c u}\right)^{2}} \\
\rho_{1,1}(\tau) & \approx \beta_{1}\left(1-e^{-\tilde{\gamma} \tau}\right), & \rho_{2,2}(\tau) & \approx \beta_{2}+e^{-\tilde{\gamma} \tau} \beta_{3} \\
\rho_{3,3}(\tau) & \approx \beta_{2}+e^{-\tilde{\gamma} \tau} \beta_{4}, & \rho_{2,3}(\tau) & \approx \alpha_{3}^{*} \rho_{1,1}^{(s)} e^{-\Gamma \tau},
\end{aligned}
$$

where

$$
\begin{aligned}
\tilde{\gamma} & =\gamma_{c u}+\gamma_{u c} \\
\Gamma & =\tilde{\gamma}+\gamma_{d} \\
\beta_{1} & =\left(\alpha_{1}+\alpha_{2}\right)\left(\rho_{1,1}^{(s)}+\rho_{2,2}^{(s)}\right) \rho_{1,1}^{(s)} \\
\beta_{2} & =\left(\alpha_{1}+\alpha_{2}\right)\left(\rho_{1,1}^{(s)}+\rho_{2,2}^{(s)}\right) \rho_{2,2}^{(s)} \\
\beta_{3} & =-\alpha_{1} \rho_{2,2}^{(s)}\left(\rho_{1,1}^{(s)}+\rho_{2,2}^{(s)}\right)+\alpha_{2}\left(\left(\rho_{1,1}^{(s)}\right)^{2}-\left(\rho_{2,2}^{(s)}\right)^{2}+\rho_{1,1}^{(s)}\left(\rho_{2,2}^{(s)}+\rho_{4,4}^{(s)}\right)\right) \\
\beta_{4} & =-\alpha_{2} \rho_{2,2}^{(s)}\left(\rho_{1,1}^{(s)}+\rho_{2,2}^{(s)}\right)+\alpha_{1}\left(\left(\rho_{1,1}^{(s)}\right)^{2}-\left(\rho_{2,2}^{(s)}\right)^{2}+\rho_{1,1}^{(s)}\left(\rho_{2,2}^{(s)}+\rho_{4,4}^{(s)}\right)\right)
\end{aligned}
$$




\section{Bibliography}

[1] D. J. Wineland. Nobel lecture: Superposition, entanglement, and raising schrödinger's cat. Rev. Mod. Phys., 85:1103-1114, Jul 2013.

[2] S. Haroche. Nobel lecture: Controlling photons in a box and exploring the quantum to classical boundary. Rev. Mod. Phys., 85:1083-1102, Jul 2013.

[3] J. I. Cirac and P. Zoller. Quantum computations with cold trapped ions. Phys. Rev. Lett., 74(20):4091, 1995.

[4] D. Jaksch, C. Bruder, J. I. Cirac, C. W. Gardiner, and P. Zoller. Cold bosonic atoms in optical lattices. Phys. Rev. Lett., 81(15):3108, 1998.

[5] J. Maze, P. Stanwix, J. Hodges, S. Hong, J. Taylor, P. Cappellaro, L. Jiang, M. G. Dutt, E. Togan, A. Zibrov, et al. Nanoscale magnetic sensing with an individual electronic spin in diamond. Nature, 455(7213):644-647, 2008.

[6] J. Aasi, J. Abadie, B. Abbott, R. Abbott, T. Abbott, M. Abernathy, C. Adams, T. Adams, P. Addesso, R. Adhikari, et al. Enhanced sensitivity of the ligo gravitational wave detector by using squeezed states of light. Nat. Photon., $7(8): 613-619,2013$.

[7] J. Cirac, P. Zoller, H. Kimble, and H. Mabuchi. Quantum state transfer and entanglement distribution among distant nodes in a quantum network. Phys. Rev. Lett., 78(16):3221, 1997.

[8] H. J. Kimble. The quantum internet. Nature, 453(7198):1023-1030, 2008.

[9] D. Jaksch, J. Cirac, P. Zoller, S. Rolston, R. Côté, and M. Lukin. Fast quantum gates for neutral atoms. Phys. Rev. Lett., 85(10):2208, 2000.

[10] E. Urban, T. A. Johnson, T. Henage, L. Isenhower, D. Yavuz, T. Walker, and M. Saffman. Observation of rydberg blockade between two atoms. Nat. Phys., 5(2):110-114, 2009.

[11] C. Monroe, D. Meekhof, B. King, W. M. Itano, and D. J. Wineland. Demonstration of a fundamental quantum logic gate. Phys. Rev. Lett., 75(25):4714, 1995 . 
[12] T. Pellizzari, S. Gardiner, J. Cirac, and P. Zoller. Decoherence, continuous observation, and quantum computing: A cavity qed model. Phys. Rev. Lett., 75(21):3788, 1995.

[13] A. Neuzner, M. Körber, O. Morin, S. Ritter, and G. Rempe. Interference and dynamics of light from a distance-controlled atom pair in an optical cavity. Nat. Photon., 10(5):303-306, 2016.

[14] J. Majer, J. Chow, J. Gambetta, J. Koch, B. Johnson, J. Schreier, L. Frunzio, D. Schuster, A. Houck, A. Wallraff, et al. Coupling superconducting qubits via a cavity bus. Nature, 449(7161):443-447, 2007.

[15] T. Monz, P. Schindler, J. T. Barreiro, M. Chwalla, D. Nigg, W. a. Coish, M. Harlander, W. Hänsel, M. Hennrich, and R. Blatt. 14-Qubit Entanglement: Creation and Coherence. Phys. Rev. Lett., 106(13):130506, March 2011.

[16] R. Barends, J. Kelly, A. Megrant, A. Veitia, D. Sank, E. Jeffrey, T. C. White, J. Mutus, A. G. Fowler, B. Campbell, et al. Superconducting quantum circuits at the surface code threshold for fault tolerance. Nature, 508(7497):500-503, 2014 .

[17] C. Monroe, R. Raussendorf, A. Ruthven, K. Brown, P. Maunz, L.-M. Duan, and J. Kim. Large-scale modular quantum-computer architecture with atomic memory and photonic interconnects. Phys. Rev. A, 89(2):022317, 2014.

[18] C. R. Monroe, R. J. Schoelkopf, and M. D. Lukin. Quantum connections. Sci. Amer., 314(5):50-57, 2016.

[19] R. W. Andrews, R. W. Peterson, T. P. Purdy, K. Cicak, R. W. Simmonds, C. A. Regal, and K. W. Lehnert. Bidirectional and efficient conversion between microwave and optical light. Nat. Phys., 10(4):321-326, 2014.

[20] H. Briegel, W. Dür, J. I. Cirac, and P. Zoller. Quantum Repeaters : The Role of Imperfect Local Operations in Quantum Communication. Phys. Rev. Lett., 81(26):5932-5935, dec 1998.

[21] S. Ritter, C. Nölleke, C. Hahn, A. Reiserer, A. Neuzner, M. Uphoff, M. Mücke, E. Figueroa, J. Bochmann, and G. Rempe. An elementary quantum network of single atoms in optical cavities. Nature, 484(7393):195-200, April 2012.

[22] I. Aharonovich, D. Englund, and M. Toth. Solid-state single-photon emitters. Nat. Photon., 10(10):631-641, 2016.

[23] P. Michler, A. Kiraz, C. Becher, W. Schoenfeld, P. Petroff, L. Zhang, E. Hu, and A. Imamoglu. A quantum dot single-photon turnstile device. Science, 290(5500):2282-2285, 2000. 
[24] A. I. Lvovsky, B. C. Sanders, and W. Tittel. Optical quantum memory. Nat. Photon., 3(12):706-714, 2009.

[25] M. Orrit and J. Bernard. Single pentacene molecules detected by fluorescence excitation in ap-terphenyl crystal. Phys. Rev. Lett., 65(21):2716-2719, 1990.

[26] A. Gruber, A. Dräbenstedt, C. Tietz, L. Fleury, J. Wrachtrup, and C. v. Borczyskowski. Scanning confocal optical microscopy and magnetic resonance on single defect centers. Science, 276(5321):2012-2014, 1997.

[27] T. Maiman. Stimulated optical radiation in ruby. Nature, 187(4736):493-494, 1960 .

[28] B. Judd. Optical absorption intensities of rare-earth ions. Phys. Rev., $127(3): 750,1962$.

[29] G. Liu and B. Jacquier. Spectroscopic properties of rare earths in optical materials, volume 83. Springer Science \& Business Media, 2006.

[30] R. Mears, L. Reekie, I. Jauncey, and D. Payne. Low-noise erbium-doped fibre amplifier operating at 1.54 $\mu \mathrm{m}$. Electron. Lett., 23(19):1026-1028, 1987.

[31] A. M. Stoneham. Theory of defects in solids. Clarendon Press, Oxford, 1975.

[32] W. E. Moerner and L. Kador. Optical detection and spectroscopy of single molecules in a solid. Phys. Rev. Lett., 62(21):2535, 1989.

[33] W. Gao, A. Imamoglu, H. Bernien, and R. Hanson. Coherent manipulation, measurement and entanglement of individual solid-state spins using optical fields. Nat. Photon., 9(6):363-373, 2015.

[34] C. Santori, D. Fattal, J. Vuckovic, G. Solomon, and Y. Yamamoto. Indistinguishable photons from a single-photon device. Nature, 419(6907):594-597, 2002 .

[35] P. Lodahl, S. Mahmoodian, and S. Stobbe. Interfacing single photons and single quantum dots with photonic nanostructures. Rev. Mod. Phys., 87(2):347, 2015.

[36] K. Hennessy, A. Badolato, M. Winger, D. Gerace, M. Atatüre, S. Gulde, S. Fält, E. L. Hu, and A. Imamoğlu. Quantum nature of a strongly coupled single quantum dot-cavity system. Nature, 445(7130):896-899, 2007.

[37] D. Englund, A. Faraon, I. Fushman, N. Stoltz, P. Petroff, and J. Vučković. Controlling cavity reflectivity with a single quantum dot. Nature, 450(7171):857861, 2007. 
[38] A. Greilich. Mode locking of electron spin coherences in singly charged quantum dots. Science, 313(5785):341-345, jul 2006.

[39] D. Press, K. D. Greve, P. L. McMahon, T. D. Ladd, B. Friess, C. Schneider, M. Kamp, S. Hfling, A. Forchel, and Y. Yamamoto. Ultrafast optical spin echo in a single quantum dot. Nat. Photon., 4(6):367-370, 2010.

[40] A. Bechtold, D. Rauch, F. Li, T. Simmet, P.-L. Ardelt, A. Regler, K. Mller, N. A. Sinitsyn, and J. J. Finley. Three-stage decoherence dynamics of an electron spin qubit in an optically active quantum dot. Nat. Phys., 11(12):1005$1008,2015$.

[41] R. Stockill, C. L. Gall, C. Matthiesen, L. Huthmacher, E. Clarke, M. Hugues, and M. Atatre. Quantum dot spin coherence governed by a strained nuclear environment. Nat. Commun., 7:12745, 2016.

[42] A. M. Zaitsev. Optical properties of diamond: a data handbook. Springer Science \& Business Media, 2001.

[43] G. Balasubramanian, P. Neumann, D. Twitchen, M. Markham, R. Kolesov, N. Mizuochi, J. Isoya, J. Achard, J. Beck, J. Tissler, V. Jacques, P. R. Hemmer, F. Jelezko, and J. Wrachtrup. Ultralong spin coherence time in isotopically engineered diamond. Nat. Mater., 8(5):383-387, 2009.

[44] F. Jelezko, T. Gaebel, I. Popa, A. Gruber, and J. Wrachtrup. Observation of Coherent Oscillations in a Single Electron Spin. Phys. Rev. Lett., 92(7):076401, 2004 .

[45] J. M. Taylor, P. Cappellaro, L. Childress, L. Jiang, D. Budker, P. R. Hemmer, A. Yacoby, R. Walsworth, and M. D. Lukin. High-sensitivity diamond magnetometer with nanoscale resolution. Nat. Phys., 4(10):810-816, 2008.

[46] I. Lovchinsky, A. Sushkov, E. Urbach, N. de Leon, S. Choi, K. De Greve, R. Evans, R. Gertner, E. Bersin, C. Müller, et al. Nuclear magnetic resonance detection and spectroscopy of single proteins using quantum logic. Science, 351(6275):836-841, 2016.

[47] G. Kucsko, P. C. Maurer, N. Y. Yao, M. Kubo, H. J. Noh, P. K. Lo, H. Park, and M. D. Lukin. Nanometre-scale thermometry in a living cell. Nature (London), 500(7460):54-8, August 2013.

[48] P. Tamarat, T. Gaebel, J. Rabeau, M. Khan, A. Greentree, H. Wilson, L. Hollenberg, S. Prawer, P. Hemmer, F. Jelezko, et al. Stark shift control of single optical centers in diamond. Phys. Rev. Lett., 97(8):083002, 2006. 
[49] A. Faraon, C. Santori, Z. Huang, V. M. Acosta, and R. G. Beausoleil. Coupling of nitrogen-vacancy centers to photonic crystal cavities in monocrystalline diamond. Phys. Rev. Lett., 109:033604, Jul 2012.

[50] D. Riedel, I. Sllner, B. J. Shields, S. Starosielec, P. Appel, E. Neu, P. Maletinsky, and R. J. Warburton. Deterministic enhancement of coherent photon generation from a nitrogen-vacancy center in ultrapure diamond. arXiv:1703.00815, 2017.

[51] D. Englund, B. Shields, K. Rivoire, F. Hatami, J. Vuckovic, H. Park, and M. D. Lukin. Deterministic coupling of a single nitrogen vacancy center to a photonic crystal cavity. Nano Lett., 1:1-10, 2010.

[52] B. J. Hausmann, B. J. Shields, Q. Quan, Y. Chu, N. P. de Leon, R. Evans, M. J. Burek, A. S. Zibrov, M. Markham, D. J. Twitchen, et al. Coupling of nv centers to photonic crystal nanobeams in diamond. Nano Lett., 13(12):5791-5796, 2013.

[53] R. Albrecht, A. Bommer, C. Deutsch, J. Reichel, and C. Becher. Coupling of a single nitrogen-vacancy center in diamond to a fiber-based microcavity. Phys. Rev. Lett., 110(24):243602, 2013.

[54] Y. Chu, N. P. de Leon, B. J. Shields, B. Hausmann, R. Evans, E. Togan, M. J. Burek, M. Markham, A. Stacey, A. S. Zibrov, A. Yacoby, D. J. Twitchen, M. Lončar, H. Park, P. Maletinsky, and M. D. Lukin. Coherent optical transitions in implanted nitrogen vacancy centers. Nano Lett., 14(4):1982-1986, 2014 .

[55] E. Neu, D. Steinmetz, J. Riedrich-Möller, S. Gsell, M. Fischer, M. Schreck, and C. Becher. Single photon emission from silicon-vacancy colour centres in chemical vapour deposition nano-diamonds on iridium. New J. Phys., 13(2):025012, 2011.

[56] I. Aharonovich and E. Neu. Diamond nanophotonics. Adv. Opt. Mater., 2(10):911-928, October 2014.

[57] G. Davies and M. F. Hamer. Optical studies of the 1.945 ev vibronic band in diamond. Proceedings of the Royal Society of London. A. Mathematical and Physical Sciences, 348(1653):285-298, 1976.

[58] C. Hepp, T. Müller, V. Waselowski, J. N. Becker, B. Pingault, H. Sternschulte, D. Steinmüller-Nethl, A. Gali, J. R. Maze, M. Atatüre, and C. Becher. Electronic structure of the silicon vacancy color center in diamond. Phys. Rev. Lett., 112(3):036405, January 2014. 
[59] L. J. Rogers, K. D. Jahnke, M. W. Doherty, A. Dietrich, L. P. McGuinness, C. Müller, T. Teraji, H. Sumiya, J. Isoya, N. B. Manson, and F. Jelezko. Electronic structure of the negatively charged silicon-vacancy center in diamond. Phys. Rev. B, 89(23):235101, June 2014.

[60] C. Hepp. Electronic structure of the silicon vacancy color center in diamond. PhD thesis, Saarbrücken, Universität des Saarlandes, Diss., 2014, 2014.

[61] L. J. Rogers, K. D. Jahnke, M. H. Metsch, A. Sipahigil, J. M. Binder, T. Teraji, H. Sumiya, J. Isoya, M. D. Lukin, P. Hemmer, and F. Jelezko. All-optical initialization, readout, and coherent preparation of single silicon-vacancy spins in diamond. Phys. Rev. Lett., 113(26):263602, 2014.

[62] B. Pingault, J. N. Becker, C. H. Schulte, C. Arend, C. Hepp, T. Godde, A. I. Tartakovskii, M. Markham, C. Becher, and M. Atatüre. All-optical formation of coherent dark states of silicon-vacancy spins in diamond. Phys. Rev. Lett., 113(26):263601, 2014.

[63] B. Pingault, D.-D. Jarausch, C. Hepp, L. Klintberg, J. N. Becker, M. Markham, C. Becher, and M. Atature. Coherent control of the silicon-vacancy spin in diamond. arXiv:1701.06848, 2017.

[64] A. Sipahigil, M. L. Goldman, E. Togan, Y. Chu, M. Markham, D. J. Twitchen, A. S. Zibrov, A. Kubanek, and M. D. Lukin. Quantum interference of single photons from remote nitrogen-vacancy centers in diamond. Phys. Rev. Lett., 108(14):143601, April 2012.

[65] H. Bernien, B. Hensen, W. Pfaff, G. Koolstra, M. S. Blok, L. Robledo, T. H. Taminiau, M. Markham, D. J. Twitchen, L. Childress, and R. Hanson. Heralded entanglement between solid-state qubits separated by three metres. Nature, 497(7447):86-90, May 2013.

[66] B. Hensen, H. Bernien, A. Dréau, A. Reiserer, N. Kalb, M. Blok, J. Ruitenberg, R. Vermeulen, R. Schouten, C. Abellán, et al. Loophole-free bell inequality violation using electron spins separated by 1.3 kilometres. Nature, 526(7575):682686, 2015.

[67] C. Hong, Z. Ou, and L. Mandel. Measurement of subpicosecond time intervals between two photons by interference. Phys. Rev. Lett., 59(18):2044-2046, 1987.

[68] T. Legero, T. Wilk, M. Hennrich, G. Rempe, and A. Kuhn. Quantum beat of two single photons. Phys. Rev. Lett., 93(7):70503, 2004.

[69] A. Kiraz, M. Ehrl, T. Hellerer, Ö. Müstecaplığlu, C. Bräuchle, and A. Zumbusch. Indistinguishable photons from a single molecule. Phys. Rev. Lett., 94(22):223602, 2005. 
[70] L.-M. Duan and C. Monroe. Colloquium: Quantum networks with trapped ions. Rev. Mod. Phys., 82(2):1209-1224, April 2010.

[71] J. Beugnon, M. Jones, J. Dingjan, B. Darquie, G. Messin, A. Browaeys, and P. Grangier. Quantum interference between two single photons emitted by independently trapped atoms. Nature, 440(7085):779-782, APR 62006.

[72] P. Maunz, D. L. Moehring, S. Olmschenk, K. C. Younge, D. N. Matsukevich, and C. Monroe. Quantum interference of photon pairs from two remote trapped atomic ions. Nat Phys, 3(8):538-541, August 2007.

[73] D. Moehring, P. Maunz, S. Olmschenk, K. Younge, D. Matsukevich, L. Duan, and C. Monroe. Entanglement of single-atom quantum bits at a distance. $\mathrm{Na}$ ture, 449(7158):68-71, 2007.

[74] H. Bernien, L. Childress, L. Robledo, M. Markham, D. J. Twitchen, and R. Hanson. Two-Photon Quantum Interference from Separate Nitrogen Vacancy Centers in Diamond. Phys. Rev. Lett., 108(4):1-5, January 2012.

[75] R. Lettow, Y. L. A. Rezus, A. Renn, G. Zumofen, E. Ikonen, S. Götzinger, and V. Sandoghdar. Quantum interference of tunably indistinguishable photons from remote organic molecules. Phys. Rev. Lett., 104(12):123605-, March 2010.

[76] E. B. Flagg, A. Muller, S. V. Polyakov, A. Ling, A. Migdall, and G. S. Solomon. Interference of single photons from two separate semiconductor quantum dots. Phys. Rev. Lett., 104(13):137401-, April 2010.

[77] R. Patel, A. Bennett, I. Farrer, C. Nicoll, D. Ritchie, and A. Shields. Twophoton interference of the emission from electrically tunable remote quantum dots. Nat. Photon., 4(9):632-635, 2010.

[78] L. Childress, J. M. Taylor, A. S. Sørensen, and M. D. Lukin. Fault-tolerant quantum communication based on solid-state photon emitters. Phys. Rev. Lett., 96(7):070504-, February 2006.

[79] M. V. G. Dutt, L. Childress, L. Jiang, E. Togan, J. Maze, F. Jelezko, A. S. Zibrov, P. R. Hemmer, and M. D. Lukin. Quantum register based on individual electronic and nuclear spin qubits in diamond. Science, 316(5829):1312-1316, June 2007.

[80] E. Togan, Y. Chu, A. S. Trifonov, L. Jiang, J. Maze, L. Childress, M. V. G. Dutt, A. S. Sø rensen, P. R. Hemmer, A. S. Zibrov, and M. D. Lukin. Quantum entanglement between an optical photon and a solid-state spin qubit. Nature (London), 466(7307):730-4, August 2010. 
[81] T. Babinec, B. Hausmann, M. Khan, Y. Zhang, J. Maze, P. Hemmer, and M. Lončar. A diamond nanowire single-photon source. Nat. Nanotechnol., 5(3):195-199, 2010.

[82] A. Faraon, P. Barclay, C. Santori, K. Fu, and R. Beausoleil. Resonant enhancement of the zero-phonon emission from a colour centre in a diamond cavity. Nat. Photon., 5(5):301-305, 2011.

[83] R. Kolesov, B. Grotz, G. Balasubramanian, R. J. Stöhr, A. A. L. Nicolet, P. R. Hemmer, F. Jelezko, and J. Wrachtrup. Waveparticle duality of single surface plasmon polaritons. Nature Phys., 5(7):470-474, May 2009.

[84] P. Siyushev, F. Kaiser, V. Jacques, I. Gerhardt, S. Bischof, H. Fedder, J. Dodson, M. Markham, D. Twitchen, F. Jelezko, et al. Monolithic diamond optics for single photon detection. Appl. Phys. Lett., 97(24):241902-241902, 2010.

[85] K.-M. C. Fu, C. Santori, P. E. Barclay, L. J. Rogers, N. B. Manson, and R. G. Beausoleil. Observation of the dynamic jahn-teller effect in the excited states of nitrogen-vacancy centers in diamond. Phys. Rev. Lett., 103:256404, Dec 2009.

[86] N. Manson, L. Rogers, M. Doherty, and L. Hollenberg. Optically induced spin polarisation of the nv- centre in diamond: role of electron-vibration interaction. arXiv:1011.2840v1, 2011.

[87] A. Batalov, V. Jacques, F. Kaiser, P. Siyushev, P. Neumann, L. J. Rogers, R. McMurtrie, N. B. Manson, F. Jelezko, and J. Wrachtrup. Low Temperature Studies of the Excited-State Structure of Negatively Charged Nitrogen-Vacancy Color Centers in Diamond. Phys. Rev. Lett., 102(19):195506, May 2009.

[88] L. C. Bassett, F. J. Heremans, C. G. Yale, B. B. Buckley, and D. D. Awschalom. Electrical Tuning of Single Nitrogen-Vacancy Center Optical Transitions Enhanced by Photoinduced Fields. Phys. Rev. Lett., 107(26):266403, December 2011.

[89] J. R. Maze, A. Gali, E. Togan, Y. Chu, A. Trifonov, E. Kaxiras, and M. D. Lukin. Properties of nitrogen-vacancy centers in diamond: the group theoretic approach. New J. Phys., 13(2):025025, 2011.

[90] C. Kurtsiefer, S. Mayer, P. Zarda, and H. Weinfurter. Stable solid-state source of single photons. Phys. Rev. Lett., 85(2):290-3, July 2000.

[91] I. Aharonovich, A. D. Greentree, and S. Prawer. Diamond photonics. Nat. Photon., 5(7):397-405, June 2011. 
[92] P. C. Maurer, G. Kucsko, C. Latta, L. Jiang, N. Y. Yao, S. D. Bennett, F. Pastawski, D. Hunger, N. Chisholm, M. Markham, et al. Room-temperature quantum bit memory exceeding one second. Science, 336(6086):1283-1286, 2012 .

[93] M. S. Grinolds, S. Hong, P. Maletinsky, L. Luan, M. D. Lukin, R. L. Walsworth, and A. Yacoby. Nanoscale magnetic imaging of a single electron spin under ambient conditions. Nature Phys., 9(4):215-219, February 2013.

[94] M. W. Doherty, V. V. Struzhkin, D. A. Simpson, L. P. McGuinness, Y. Meng, A. Stacey, T. J. Karle, R. J. Hemley, N. B. Manson, L. C. L. Hollenberg, and S. Prawer. Electronic Properties and Metrology Applications of the Diamond NV Center under Pressure. Phys. Rev. Lett., 112(4):047601, January 2014.

[95] F. Dolde, H. Fedder, M. W. Doherty, T. Nöbauer, F. Rempp, G. Balasubramanian, T. Wolf, F. Reinhard, L. C. L. Hollenberg, F. Jelezko, and J. Wrachtrup. Electric-field sensing using single diamond spins. Nature Phys., 7(6):459-463, April 2011.

[96] T. van der Sar, Z. H. Wang, M. S. Blok, H. Bernien, T. H. Taminiau, D. M. Toyli, D. A. Lidar, D. D. Awschalom, R. Hanson, and V. V. Dobrovitski. Decoherence-protected quantum gates for a hybrid solid-state spin register. $\mathrm{Na}$ ture (London), 484(7392):82-6, April 2012.

[97] N. B. Manson, J. P. Harrison, and M. J. Sellars. Nitrogen-vacancy center in diamond: Model of the electronic structure and associated dynamics. Phys. Rev. B, 74(10):104303, September 2006.

[98] A. Batalov, C. Zierl, T. Gaebel, P. Neumann, I.-Y. Chan, G. Balasubramanian, P. R. Hemmer, F. Jelezko, and J. Wrachtrup. Temporal Coherence of Photons Emitted by Single Nitrogen-Vacancy Defect Centers in Diamond Using Optical Rabi-Oscillations. Phys. Rev. Lett., 100(7):077401, February 2008.

[99] L. Robledo, H. Bernien, T. van der Sar, and R. Hanson. Spin dynamics in the optical cycle of single nitrogen-vacancy centres in diamond. New J. Phys., 13(2):025013, February 2011.

[100] D. M. Toyli, D. J. Christle, A. Alkauskas, B. B. Buckley, C. G. Van de Walle, and D. D. Awschalom. Measurement and Control of Single Nitrogen-Vacancy Center Spins above 600 K. Phys. Rev. X, 2(3):031001, July 2012.

[101] F. Kaiser, V. Jacques, A. Batalov, P. Siyushev, F. Jelezko, and J. Wrachtrup. Polarization properties of single photons emitted by nitrogen-vacancy defect in diamond at low temperature. arxiv preprint arXiv:0906.3426, 2009. 
[102] L. Robledo, H. Bernien, I. van Weperen, and R. Hanson. Control and Coherence of the Optical Transition of Single Nitrogen Vacancy Centers in Diamond. Phys. Rev. Lett., 105(17):177403, October 2010.

[103] C. Cohen-Tannoudji, J. Dupont-Roc, and G. Grynberg. Atom-Photon Interactions: Basic Processes and Applications. Wiley-VCH, New York, first edition, 1992.

[104] M. L. Goldman, M. W. Doherty, A. Sipahigil, N. Y. Yao, S. D. Bennett, N. B. Manson, A. Kubanek, and M. D. Lukin. State-selective intersystem crossing in nitrogen-vacancy centers. Phys. Rev. B, 91:165201, Apr 2015.

[105] M. W. Doherty, N. B. Manson, P. Delaney, F. Jelezko, J. Wrachtrup, and L. C. Hollenberg. The nitrogen-vacancy colour centre in diamond. Phys. Rep., 528(1):1-45, 2013.

[106] G. D. Fuchs, V. V. Dobrovitski, R. Hanson, A. Batra, C. D. Weis, T. Schenkel, and D. D. Awschalom. Excited-State Spectroscopy Using Single Spin Manipulation in Diamond. Phys. Rev. Lett., 101(11):117601, September 2008.

[107] L. J. Rogers, R. L. McMurtrie, M. J. Sellars, and N. B. Manson. Time-averaging within the excited state of the nitrogen-vacancy centre in diamond. New J. Phys., 11(6):063007, June 2009.

[108] M. W. Doherty, N. B. Manson, P. Delaney, and L. C. L. Hollenberg. The negatively charged nitrogen-vacancy centre in diamond: the electronic solution. New J. Phys., 13(2):025019, 2011.

[109] V. M. Huxter, T. A. A. Oliver, D. Budker, and G. R. Fleming. Vibrational and electronic dynamics of nitrogenvacancy centres in diamond revealed by twodimensional ultrafast spectroscopy. Nature Phys., 9(11):744-749, September 2013.

[110] P. Kehayias, M. W. Doherty, D. English, R. Fischer, A. Jarmola, K. Jensen, N. Leefer, P. Hemmer, N. B. Manson, and D. Budker. Infrared absorption band and vibronic structure of the nitrogen-vacancy center in diamond. Phys. Rev. B, 88(16):165202, October 2013.

[111] V. M. Acosta, A. Jarmola, E. Bauch, and D. Budker. Optical properties of the nitrogen-vacancy singlet levels in diamond. Phys. Rev. B, 82(20):201202, November 2010.

[112] A. Lenef and S. C. Rand. Electronic structure of the N-V center in diamond: Theory. Phys. Rev. B, 53(20):13441-13455, May 1996. 
[113] L. C. Bassett, F. J. Heremans, D. J. Christle, C. G. Yale, G. Burkard, B. B. Buckley, and D. D. Awschalom. Ultrafast optical control of orbital and spin dynamics in a solid-state defect. Science, 345:1333, 2014.

[114] A. Bosak and M. Krisch. Phonon density of states probed by inelastic x-ray scattering. Phys. Rev. B, 72(22):224305, December 2005.

[115] G. Davies. Vibronic spectra in diamond. Journal of Physics C: Solid State Physics, 7(20):3797, October 1974.

[116] P. Ovartchaiyapong, L. M. A. Pascal, B. A. Myers, P. Lauria, and A. C. Bleszynski Jayich. High quality factor single-crystal diamond mechanical resonators. Appl. Phys. Lett., 101(16):163505, 2012.

[117] Y. Tao, J. M. Boss, B. A. Moores, and C. L. Degen. Single-crystal diamond nanomechanical resonators with quality factors exceeding one million. Nat. Commun., 5, 2014.

[118] K. V. Kepesidis, S. D. Bennett, S. Portolan, M. D. Lukin, and P. Rabl. Phonon cooling and lasing with nitrogen-vacancy centers in diamond. Phys. Rev. B, 88(6):064105, 2013.

[119] E. R. MacQuarrie, T. A. Gosavi, N. R. Jungwirth, S. A. Bhave, and G. D. Fuchs. Mechanical Spin Control of Nitrogen-Vacancy Centers in Diamond. Phys. Rev. Lett., 111(22):227602, November 2013.

[120] P. Ovartchaiyapong, K. W. Lee, B. A. Myers, and A. C. Bleszynski Jayich. Dynamic strain-mediated coupling of a single diamond spin to a mechanical resonator. Nat. Commun., 5:4429, January 2014.

[121] S. D. Bennett, N. Y. Yao, J. Otterbach, P. Zoller, P. Rabl, and M. D. Lukin. Phonon-induced spin-spin interactions in diamond nanostructures: application to spin squeezing. Phys. Rev. Lett., 110(15):156402, 2013.

[122] E. Togan, Y. Chu, A. S. Trifonov, L. Jiang, J. Maze, L. Childress, M. V. G. Dutt, A. S. Sørensen, P. R. Hemmer, A. S. Zibrov, and M. D. Lukin. Quantum entanglement between an optical photon and a solid-state spin qubit. Nature, 466(7307):730-734, 2010.

[123] W. Pfaff, B. J. Hensen, H. Bernien, S. B. van Dam, M. S. Blok, T. H. Taminiau, M. J. Tiggelman, R. N. Schouten, M. Markham, D. J. Twitchen, and R. Hanson. Unconditional quantum teleportation between distant solid-state quantum bits. Science, 345(6196):532-535, 2014. 
[124] J. P. Goss, R. Jones, S. J. Breuer, P. R. Briddon, and S. Öberg. The twelveline $1.682 \mathrm{eV}$ luminescence center in diamond and the vacancy-silicon complex. Phys. Rev. Lett., 77(14):3041, 1996.

[125] A. Gali and J. R. Maze. Ab initio study of the split silicon-vacancy defect in diamond: Electronic structure and related properties. Phys. Rev. B, 88(23):235205, December 2013.

[126] T. Müller, C. Hepp, B. Pingault, E. Neu, S. Gsell, M. Schreck, H. Sternschulte, D. Steinmüller-Nethl, C. Becher, and M. Atatüre. Optical signatures of siliconvacancy spins in diamond. Nat. Comm., 5:3328, 2014.

[127] L. J. Rogers, K. D. Jahnke, T. Teraji, L. Marseglia, C. Müller, B. Naydenov, H. Schauffert, C. Kranz, J. Isoya, L. P. McGuinness, and F. Jelezko. Multiple intrinsically identical single-photon emitters in the solid state. Nat. Commun., 5:4739, August 2014.

[128] H. Sternschulte, K. Thonke, R. Sauer, P. C. Münzinger, and P. Michler. 1.681eV luminescence center in chemical-vapor-deposited homoepitaxial diamond films. Phys. Rev. B, 50(19):14554-14560, November 1994.

[129] P. Siyushev, H. Pinto, M. Vörös, A. Gali, F. Jelezko, and J. Wrachtrup. Optically Controlled Switching of the Charge State of a Single NitrogenVacancy Center in Diamond at Cryogenic Temperatures. Phys. Rev. Lett., 110(16):167402, April 2013.

[130] M. J. Burek, N. P. de Leon, B. J. Shields, B. J. Hausmann, Y. Chu, Q. Quan, A. S. Zibrov, H. Park, M. D. Lukin, and M. Lončar. Free-standing mechanical and photonic nanostructures in single-crystal diamond. Nano Lett., 12(12):6084-6089, 2012.

[131] J. Riedrich-Möller, L. Kipfstuhl, C. Hepp, E. Neu, C. Pauly, F. Mücklich, A. Baur, M. Wandt, S. Wolff, M. Fischer, S. Gsell, M. Schreck, and C. Becher. One- and two-dimensional photonic crystal microcavities in single crystal diamond. Nat. Nanotechnol., 7(1):69-74, January 2012.

[132] J. C. Lee, I. Aharonovich, A. P. Magyar, F. Rol, and E. L. Hu. Coupling of silicon-vacancy centers to a single crystal diamond cavity. Opt. Express, 20(8):8891, April 2012.

[133] A. Kuhn, M. Hennrich, and G. Rempe. Deterministic single-photon source for distributed quantum networking. Phys. Rev. Lett., 89(6):067901, 2002.

[134] T. Tiecke, J. D. Thompson, N. P. de Leon, L. Liu, V. Vuletić, and M. D. Lukin. Nanophotonic quantum phase switch with a single atom. Nature, 508(7495):241-244, 2014. 
[135] P. Kok, W. J. Munro, K. Nemoto, T. C. Ralph, J. P. Dowling, and G. Milburn. Linear optical quantum computing with photonic qubits. Rev. Mod. Phys., $79(1): 135,2007$.

[136] L. Childress, J. M. Taylor, A. S. Sørensen, and M. D. Lukin. Fault-tolerant quantum repeaters with minimal physical resources and implementations based on single-photon emitters. Phys. Rev. A, 72(5):052330, 2005.

[137] A. M. Edmonds, M. E. Newton, P. M. Martineau, D. J. Twitchen, and S. D. Williams. Electron paramagnetic resonance studies of silicon-related defects in diamond. Phys. Rev. B, 77(24):245205, 2008.

[138] J. R. Weber, W. F. Koehl, J. B. Varley, a. Janotti, B. B. Buckley, C. G. Van de Walle, and D. D. Awschalom. Quantum computing with defects. Proc. Natl. Acad. Sci. U.S.A., 107(19):8513-8, May 2010.

[139] L. Childress, R. Walsworth, and M. Lukin. Atom-like crystal defects: From quantum computers to biological sensors. Phys. Today, 67(10):38-43, 2014.

[140] T. D. Ladd, F. Jelezko, R. Laflamme, Y. Nakamura, C. Monroe, and J. L. O'Brien. Quantum computers. Nature, 464(7285):45-53, 2010.

[141] I. Aharonovich, S. Castelletto, D. A. Simpson, C.-H. Su, A. D. Greentree, and S. Prawer. Diamond-based single-photon emitters. Rep. Prog. Phys., 74(7):076501, 2011.

[142] A. Sipahigil, K. D. Jahnke, L. J. Rogers, T. Teraji, J. Isoya, A. S. Zibrov, F. Jelezko, and M. D. Lukin. Indistinguishable photons from separated siliconvacancy centers in diamond. Phys. Rev. Lett., 113:113602, Sep 2014.

[143] Y. L. Lim, S. D. Barrett, A. Beige, P. Kok, and L. C. Kwek. Repeat-untilsuccess quantum computing using stationary and flying qubits. Phys. Rev. A, 73:012304, Jan 2006.

[144] J. L. O'Brien. Optical quantum computing. Science, 318(5856):1567-1570, 2007.

[145] E. Knill, R. Laflamme, and G. J. Milburn. A scheme for efficient quantum computation with linear optics. Nature, 409(6816):46-52, 2001.

[146] W. B. Gao, P. Fallahi, E. Togan, J. Miguel-Sanchez, and A. Imamoglu. Observation of entanglement between a quantum dot spin and a single photon. Nature, 491(7424):426-430, 2012. 
[147] K. De Greve, L. Yu, P. L. McMahon, J. S. Pelc, C. M. Natarajan, N. Y. Kim, E. Abe, S. Maier, C. Schneider, M. Kamp, S. Höfling, R. H. Hadfield, A. Forchel, M. M. Fejer, and Y. Yamamoto. Quantum-dot spin-photon entanglement via frequency downconversion to telecom wavelength. Nature, 491(7424):421-425, 2012 .

[148] U. F. S. D’Haenens-Johansson, A. M. Edmonds, B. L. Green, M. E. Newton, G. Davies, P. M. Martineau, R. U. A. Khan, and D. J. Twitchen. Optical properties of the neutral silicon split-vacancy center in diamond. Phys. Rev. B, 84:245208, Dec 2011.

[149] F. S. Ham. Dynamical jahn-teller effect in paramagnetic resonance spectra: orbital reduction factors and partial quenching of spin-orbit interaction. Phys. Rev., 138(6A):A1727, 1965.

[150] G. Fischer. Vibronic coupling: The interaction between the electronic and nuclear motions. Academic Press, London, 1984.

[151] M. L. Goldman, A. Sipahigil, M. W. Doherty, N. Y. Yao, S. D. Bennett, M. Markham, D. J. Twitchen, N. B. Manson, A. Kubanek, and M. D. Lukin. Phonon-induced population dynamics and intersystem crossing in nitrogenvacancy centers. Phys. Rev. Lett., 114:145502, Apr 2015.

[152] C. D. Clark, H. Kanda, I. Kiflawi, and G. Sittas. Silicon defects in diamond. Phys. Rev. B, 51:16681-16688, Jun 1995.

[153] E. Neu, C. Hepp, M. Hauschild, S. Gsell, M. Fischer, H. Sternschulte, D. Steinmüller-Nethl, M. Schreck, and C. Becher. Low-temperature investigations of single silicon vacancy colour centres in diamond. New J. Phys., 15(4):043005, April 2013.

[154] J. Riedrich-Möller, C. Arend, C. Pauly, F. Mücklich, M. Fischer, S. Gsell, M. Schreck, and C. Becher. Deterministic coupling of a single silicon-vacancy color center to a photonic crystal cavity in diamond. Nano Lett., 14(9):52815287, 2014.

[155] D. M. Toyli, D. J. Christle, A. Alkauskas, B. B. Buckley, C. G. Van de Walle, and D. D. Awschalom. Measurement and control of single nitrogen-vacancy center spins above 600 k. Phys. Rev. X, 2:031001, Jul 2012.

[156] E. Neu, R. Albrecht, M. Fischer, S. Gsell, M. Schreck, and C. Becher. Electronic transitions of single silicon vacancy centers in the near-infrared spectral region. Phys. Rev. B, 85(24), June 2012. 
[157] G. Waldherr, J. Beck, M. Steiner, P. Neumann, A. Gali, T. Frauenheim, F. Jelezko, and J. Wrachtrup. Dark states of single nitrogen-vacancy centers in diamond unraveled by single shot nmr. Phys. Rev. Lett., 106:157601, Apr 2011.

[158] M. W. Doherty, V. M. Acosta, A. Jarmola, M. S. J. Barson, N. B. Manson, D. Budker, and L. C. L. Hollenberg. Temperature shifts of the resonances of the NV center in diamond. Phys. Rev. B, 90(4):041201, July 2014.

[159] T. Sato, K. Ohashi, T. Sudoh, K. Haruna, and H. Maeta. Thermal expansion of a high purity synthetic diamond single crystal at low temperatures. Phys. Rev. B, 65(9):092102, February 2002.

[160] A. A. Maradudin. Advances in Research and Applications. Number 18 in Solid State Physics. Academic, New York, 1966.

[161] T. A. Abtew, Y. Y. Sun, B.-C. Shih, P. Dev, S. B. Zhang, and P. Zhang. Dynamic jahn-teller effect in the $\mathrm{nv}^{-}$center in diamond. Phys. Rev. Lett., 107:146403, Sep 2011.

[162] R. Englman. The Jahn-Teller effect in molecules and crystals. Wiley, New York, 1972.

[163] H. C. Longuet-Higgins. Advances in Spectroscopy 2. Wiley-Interscience, New York, 1961.

[164] Y. Yamamoto and A. Imamoglu. Mesoscopic quantum optics. Mesoscopic Quantum Optics, published by John Wiley 6 Sons, Inc., New York, 1999., 1, 1999 .

[165] D. Kleppner. Inhibited spontaneous emission. Phys. Rev. Lett., 47(4):233, 1981.

[166] R. G. Hulet, E. S. Hilfer, and D. Kleppner. Inhibited spontaneous emission by a rydberg atom. Phys. Rev. Lett., 55:2137-2140, Nov 1985.

[167] P. Lodahl, A. F. Van Driel, I. S. Nikolaev, A. Irman, K. Overgaag, D. Vanmaekelbergh, and W. L. Vos. Controlling the dynamics of spontaneous emission from quantum dots by photonic crystals. Nature, 430(7000):654-657, 2004.

[168] A. A. Houck, J. A. Schreier, B. R. Johnson, J. M. Chow, J. Koch, J. M. Gambetta, D. I. Schuster, L. Frunzio, M. H. Devoret, S. M. Girvin, and R. J. Schoelkopf. Controlling the spontaneous emission of a superconducting transmon qubit. Phys. Rev. Lett., 101:080502, Aug 2008.

[169] A. Albrecht, A. Retzker, F. Jelezko, and M. B. Plenio. Coupling of nitrogen vacancy centres in nanodiamonds by means of phonons. New J. Phys., 15(8):083014, 2013. 
[170] M. J. Burek, D. Ramos, P. Patel, I. W. Frank, and M. Lončar. Nanomechanical resonant structures in single-crystal diamond. Appl. Phys. Lett., 103(13):131904, September 2013.

[171] A. H. Safavi-Naeini, J. T. Hill, S. Meenehan, J. Chan, S. Gröblacher, and O. Painter. Two-dimensional phononic-photonic band gap optomechanical crystal cavity. Phys. Rev. Lett., 112:153603, Apr 2014.

[172] J. Gomis-Bresco, D. Navarro-Urrios, M. Oudich, S. El-Jallal, A. Griol, D. Puerto, E. Chavez, Y. Pennec, B. Djafari-Rouhani, F. Alzina, A. Martínez, and C. M. S. Torres. A one-dimensional optomechanical crystal with a complete phononic band gap. Nat. Commun., 5, July 2014.

[173] L. Kipfstuhl, F. Guldner, J. Riedrich-Möller, and C. Becher. Modeling of optomechanical coupling in a phoxonic crystal cavity in diamond. Opt. Express, 22(10):12410-12423, 2014.

[174] E. Yablonovitch. Inhibited spontaneous emission in solid-state physics and electronics. Phys. Rev. Lett., 58:2059-2062, May 1987.

[175] J. L. O'Brien, A. Furusawa, and J. Vučković. Photonic quantum technologies. Nat. Photon., 3(12):687-695, 2009.

[176] L. J. Rogers, M. W. Doherty, M. S. J. Barson, S. Onoda, T. Ohshima, and N. B. Manson. Singlet levels of the $\mathrm{NV}^{\wedge}\{-\} \$$ centre in diamond. New J. Phys., 17(1):013048, 2014.

[177] C. Lo. Natural colorless type IaB diamond with silicon-vacancy defect center. Gems and Gemology, L:293, 2014.

[178] J. L. Zhang, H. Ishiwata, T. M. Babinec, M. Radulaski, K. Mller, K. G. Lagoudakis, C. Dory, J. Dahl, R. Edgington, V. Soulire, G. Ferro, A. A. Fokin, P. R. Schreiner, Z.-X. Shen, N. A. Melosh, and J. Vukovi. Hybrid group iv nanophotonic structures incorporating diamond silicon-vacancy color centers. Nano Lett., 16(1):212-217, 2016.

[179] S. Tamura, G. Koike, A. Komatsubara, T. Teraji, S. Onoda, L. P. McGuinness, L. Rogers, B. Naydenov, E. Wu, L. Yan, et al. Array of bright silicon-vacancy centers in diamond fabricated by low-energy focused ion beam implantation. Appl. Phys. Express, 7(11):115201, 2014.

[180] C. Wang, C. Kurtsiefer, H. Weinfurter, and B. Burchard. Single photon emission from SiV centres in diamond produced by ion implantation. J. Phys. B, 39(1):37, 2006. 
[181] K. D. Jahnke, A. Sipahigil, J. M. Binder, M. W. Doherty, M. Metsch, L. J. Rogers, N. B. Manson, M. D. Lukin, and F. Jelezko. Electron-phonon processes of the silicon-vacancy centre in diamond. New J. Phys., 17(4):043011, 2015.

[182] J. F. Ziegler, M. D. Ziegler, and J. P. Biersack. Srim-the stopping and range of ions in matter (2010). Nucl. Instrum. Meth. B, 268(11):1818-1823, 2010.

[183] M. Hauf, B. Grotz, B. Naydenov, M. Dankerl, S. Pezzagna, J. Meijer, F. Jelezko, J. Wrachtrup, M. Stutzmann, F. Reinhard, et al. Chemical control of the charge state of nitrogen-vacancy centers in diamond. Phys. Rev. B, 83(8):081304, 2011.

[184] G. Davies, S. C. Lawson, A. T. Collins, A. Mainwood, and S. J. Sharp. Vacancyrelated centers in diamond. Phys. Rev. B, 46(20):13157, 1992.

[185] P. Deák, B. Aradi, M. Kaviani, T. Frauenheim, and A. Gali. Formation of NV centers in diamond: A theoretical study based on calculated transitions and migration of nitrogen and vacancy related defects. Phys. Rev. B, 89(7):075203, 2014 .

[186] V. Acosta, E. Bauch, M. Ledbetter, C. Santori, K.-M. Fu, P. Barclay, R. Beausoleil, H. Linget, J. Roch, F. Treussart, et al. Diamonds with a high density of nitrogen-vacancy centers for magnetometry applications. Phys. Rev. B, 80(11):115202, 2009.

[187] T. Yamamoto, C. Müller, L. P. McGuinness, T. Teraji, B. Naydenov, S. Onoda, T. Ohshima, J. Wrachtrup, F. Jelezko, and J. Isoya. Strongly coupled diamond spin qubits by molecular nitrogen implantation. Phys. Rev. B, 88(20):201201, 2013.

[188] C. D. Clark and C. Dickerson. The $1.681 \mathrm{eV}$ centre in polycrystalline diamond. Surf. Coat. Tech., 47(1):336-343, 1991.

[189] S. Pezzagna, B. Naydenov, F. Jelezko, J. Wrachtrup, and J. Meijer. Creation efficiency of nitrogen-vacancy centres in diamond. New J. Phys., 12(6):065017, June 2010.

[190] J. Orwa, C. Santori, K. Fu, B. Gibson, D. Simpson, I. Aharonovich, A. Stacey, A. Cimmino, P. Balog, M. Markham, et al. Engineering of nitrogen-vacancy color centers in high purity diamond by ion implantation and annealing. $J$. Appl. Phys., 109(8):083530, 2011.

[191] K. J. Vahala. Optical microcavities. Nature, 424(6950):839-846, 2003.

[192] Y. Li, P. C. Humphreys, G. J. Mendoza, and S. C. Benjamin. Resource costs for fault-tolerant linear optical quantum computing. Phys. Rev. X, 5:041007, Oct 2015 . 
[193] Y. Chu and M. D. Lukin. Quantum optics with nitrogen-vacancy centers in diamond. arXiv preprint arXiv:1504.05990, 2015.

[194] B. J. M. Hausmann, B. Shields, Q. Quan, P. Maletinsky, M. McCutcheon, J. T. Choy, T. M. Babinec, A. Kubanek, A. Yacoby, M. D. Lukin, and M. Loncar. Integrated diamond networks for quantum nanophotonics. Nano Lett., 12(3):1578-82, March 2012.

[195] M. J. Burek, Y. Chu, M. S. Liddy, P. Patel, J. Rochman, S. Meesala, W. Hong, Q. Quan, M. D. Lukin, and M. Lončar. High quality-factor optical nanocavities in bulk single-crystal diamond. Nat. Commun., 5:5718, 2014.

[196] J. C. Lee, I. Aharonovich, A. P. Magyar, F. Rol, and E. L. Hu. Coupling of silicon-vacancy centers to a single crystal diamond cavity. Opt. Express, 20(8):8891-8897, 2012.

[197] A. Sipahigil, R. E. Evans, D. D. Sukachev, M. J. Burek, J. Borregaard, M. K. Bhaskar, C. T. Nguyen, J. L. Pacheco, H. A. Atikian, C. Meuwly, R. M. Camacho, F. Jelezko, E. Bielejec, H. Park, M. Lončar, and M. D. Lukin. An integrated diamond nanophotonics platform for quantum-optical networks. Science, 354(6314):847-850, oct 2016.

[198] D. E. Chang, V. Vuletić, and M. D. Lukin. Quantum nonlinear optics [mdash] photon by photon. Nat. Photon., 8(9):685-694, 2014.

[199] A. Reiserer, N. Kalb, G. Rempe, and S. Ritter. A quantum gate between a flying optical photon and a single trapped atom. Nature, 508(7495):237-240, 2014 .

[200] A. Javadi, I. Sollner, M. Arcari, S. L. Hansen, L. Midolo, S. Mahmoodian, G. Kirsanske, T. Pregnolato, E. H. Lee, J. D. Song, S. Stobbe, and P. Lodahl. Single-photon non-linear optics with a quantum dot in a waveguide. Nat. Commun., 6:8655, Oct 2015.

[201] S. Sun, H. Kim, G. S. Solomon, and E. Waks. A quantum phase switch between a single solid-state spin and a photon. Nat. Nanotechnol., 11:539544, 2016.

[202] J. Mlynek, A. Abdumalikov, C. Eichler, and A. Wallraff. Observation of dicke superradiance for two artificial atoms in a cavity with high decay rate. Nat. Commun., 5, 2014.

[203] B. Casabone, A. Stute, K. Friebe, B. Brandstätter, K. Schüppert, R. Blatt, and T. Northup. Heralded entanglement of two ions in an optical cavity. Phys. Rev. Lett., 111(10):100505, 2013. 
[204] A. Badolato, K. Hennessy, M. Atatüre, J. Dreiser, E. Hu, P. M. Petroff, and A. Imamoğlu. Deterministic coupling of single quantum dots to single nanocavity modes. Science, 308(5725):1158-1161, 2005.

[205] R. E. Evans, A. Sipahigil, D. D. Sukachev, A. S. Zibrov, and M. D. Lukin. Narrow-linewidth homogeneous optical emitters in diamond nanostructures via silicon ion implantation. Phys. Rev. Appl., 5(4):044010, 2016.

[206] D. E. Chang, A. S. Sørensen, E. A. Demler, and M. D. Lukin. A single-photon transistor using nanoscale surface plasmons. Nat. Phys., 3(11):807-812, 2007.

[207] P. R. Rice and H. J. Carmichael. Single-atom cavity-enhanced absorption. i. photon statistics in the bad-cavity limit. IEEE J. Quantum Elect., 24(7):1351$1366,1988$.

[208] J. N. Becker, J. Grlitz, C. Arend, M. Markham, and C. Becher. Ultrafast alloptical coherent control of single silicon vacancy colour centres in diamond. Nat. Commun., 7:13512, nov 2016 .

[209] T. Tiecke, K. Nayak, J. Thompson, T. Peyronel, N. de Leon, V. Vuletić, and M. Lukin. Efficient fiber-optical interface for nanophotonic devices. Optica, $2(2): 70-75,2015$.

[210] M. J. Burek, C. Meuwly, R. E. Evans, M. K. Bhaskar, A. Sipahigil, S. Meesala, D. D. Sukachev, C. T. Nguyen, J. L. Pacheco, E. Bielejec, M. D. Lukin, and M. Lonar. A fiber-coupled diamond quantum nanophotonic interface. arXiv:1612.05285, 2016.

[211] G. Fernandez, T. Volz, R. Desbuquois, A. Badolato, and A. Imamoglu. Optically tunable spontaneous raman fluorescence from a single self-assembled ingaas quantum dot. Phys. Rev. Lett., 103(8):087406, 2009.

[212] T. M. Sweeney, S. G. Carter, A. S. Bracker, M. Kim, C. S. Kim, L. Yang, P. M. Vora, P. G. Brereton, E. R. Cleveland, and D. Gammon. Cavity-stimulated raman emission from a single quantum dot spin. Nat. Photon., 8(6):442-447, 2014 .

[213] A. Delteil, Z. Sun, W.-b. Gao, E. Togan, S. Faelt, and A. Imamoğlu. Generation of heralded entanglement between distant hole spins. Nat. Phys., 12:218-223, 2015 .

[214] C. Cabrillo, J. Cirac, P. Garcia-Fernandez, and P. Zoller. Creation of entangled states of distant atoms by interference. Phys. Rev. A, 59(2):1025, 1999. 
[215] R. Wiegner, S. Oppel, D. Bhatti, J. von Zanthier, and G. Agarwal. Simulating superradiance from higher-order-intensity-correlation measurements: Single atoms. Phys. Rev. A, 92(3):033832, 2015.

[216] N. Kalb, A. A. Reiserer, P. C. Humphreys, J. J. W. Bakermans, S. J. Kamerling, N. H. Nickerson, S. C. Benjamin, D. J. Twitchen, M. Markham, and R. Hanson. Entanglement distillation between solid-state quantum network nodes. arXiv:1703.03244, 2017.

[217] E. Togan, Y. Chu, A. Imamoglu, and M. D. Lukin. Laser cooling and real-time measurement of the nuclear spin environment of a solid-state qubit. Nature (London), 478(7370):497-501, October 2011.

[218] D. A. Golter, K. N. Dinyari, and H. Wang. Nuclear-spin-dependent coherent population trapping of single nitrogen-vacancy centers in diamond. Phys. Rev. A, 87:035801, Mar 2013.

[219] Y. Chu, M. Markham, D. J. Twitchen, and M. D. Lukin. All-optical control of a single electron spin in diamond. Phys. Rev. A, 91:021801, Feb 2015.

[220] G. de Lange, Z. H. Wang, D. Ristè, V. V. Dobrovitski, and R. Hanson. Universal dynamical decoupling of a single solid-state spin from a spin bath. Science, 330(6000):60-3, October 2010.

[221] A. Imamoglu, D. D. Awschalom, G. Burkard, D. P. DiVincenzo, D. Loss, M. Sherwin, A. Small, et al. Quantum information processing using quantum dot spins and cavity qed. Phys. Rev. Lett., 83(20):4204, 1999.

[222] S. Meesala, Y.-I. Sohn, H. A. Atikian, S. Kim, M. J. Burek, J. T. Choy, and M. Lončar. Enhanced strain coupling of nitrogen-vacancy spins to nanoscale diamond cantilevers. Phys. Rev. Applied, 5(3), mar 2016.

[223] G. Feher. Electron spin resonance experiments on donors in silicon. i. electronic structure of donors by the electron nuclear double resonance technique. Phys. Rev., 114:1219-1244, Jun 1959.

[224] K. J. Morse, R. J. S. Abraham, H. Riemann, N. V. Abrosimov, P. Becker, H.-J. Pohl, M. L. W. Thewalt, and S. Simmons. A photonic platform for donor spin qubits in silicon. arXiv:1606.03488, 2016.

[225] J. P. Goss, P. R. Briddon, M. J. Rayson, S. J. Sque, and R. Jones. Vacancyimpurity complexes and limitations for implantation doping of diamond. Phys. Rev. B, 72(3):035214, 2005. 
[226] Y. N. Palyanov, I. N. Kupriyanov, Y. M. Borzdov, and N. V. Surovtsev. Germanium: a new catalyst for diamond synthesis and a new optically active impurity in diamond. Sci. Rep., 5:14789, 2015.

[227] T. Iwasaki, F. Ishibashi, Y. Miyamoto, Y. Doi, S. Kobayashi, T. Miyazaki, K. Tahara, K. D. Jahnke, L. J. Rogers, B. Naydenov, et al. Germanium-vacancy single color centers in diamond. Sci. Rep., 5:12882, 2015.

[228] E. A. Ekimov, S. Lyapin, K. N. Boldyrev, M. V. Kondrin, R. Khmelnitskiy, V. A. Gavva, T. V. Kotereva, and M. N. Popova. Germanium-vacancy color center in isotopically enriched diamonds synthesized at high pressures. JETP Lett., 102(11):701-706, 2015.

[229] Y. N. Palyanov, I. N. Kupriyanov, Y. M. Borzdov, A. F. Khokhryakov, and N. V. Surovtsev. High-pressure synthesis and characterization of ge-doped single crystal diamond. Cryst. Growth Des., 16(6):3510-3518, 2016.

[230] M. K. Bhaskar, D. D. Sukachev, A. Sipahigil, R. E. Evans, M. J. Burek, C. T. Nguyen, L. J. Rogers, P. Siyushev, M. H. Metsch, H. Park, F. Jelezko, M. Lonar, and M. D. Lukin. Quantum nonlinear optics with a germanium-vacancy color center in a nanoscale diamond waveguide. arXiv:1612.03036, 2016.

[231] J.-P. Tetienne, L. Rondin, P. Spinicelli, M. Chipaux, T. Debuisschert, J.-F. Roch, and V. Jacques. Magnetic-field-dependent photodynamics of single NV defects in diamond: an application to qualitative all-optical magnetic imaging. New J. Phys., 14(10):103033, October 2012.

[232] L. Robledo, L. Childress, H. Bernien, B. Hensen, P. F. A. Alkemade, and R. Hanson. High-fidelity projective read-out of a solid-state spin quantum register. Nature (London), 477(7366):574-578, September 2011.

[233] T. Teraji, T. Taniguchi, S. Koizumi, K. Watanabe, M. Liao, Y. Koide, and J. Isoya. Chemical vapor deposition of 12c isotopically enriched polycrystalline diamond. Jpn. J. Appl. Phys., 51(9R):090104, September 2012.

[234] E. Neu, M. Agio, and C. Becher. Photophysics of single silicon vacancy centers in diamond: implications for single photon emission. Opt. Express, 20(18):1995619971, 2012.

[235] W. K. Wootters. Entanglement of formation of an arbitrary state of two qubits. Phys. Rev. Lett., 80:2245-2248, Mar 1998.

[236] C. W. Chou, H. de Riedmatten, D. Felinto, S. V. Polyakov, S. J. van Enk, and H. J. Kimble. Measurement-induced entanglement for excitation stored in remote atomic ensembles. Nature, 438(7069):828-832, 122005. 